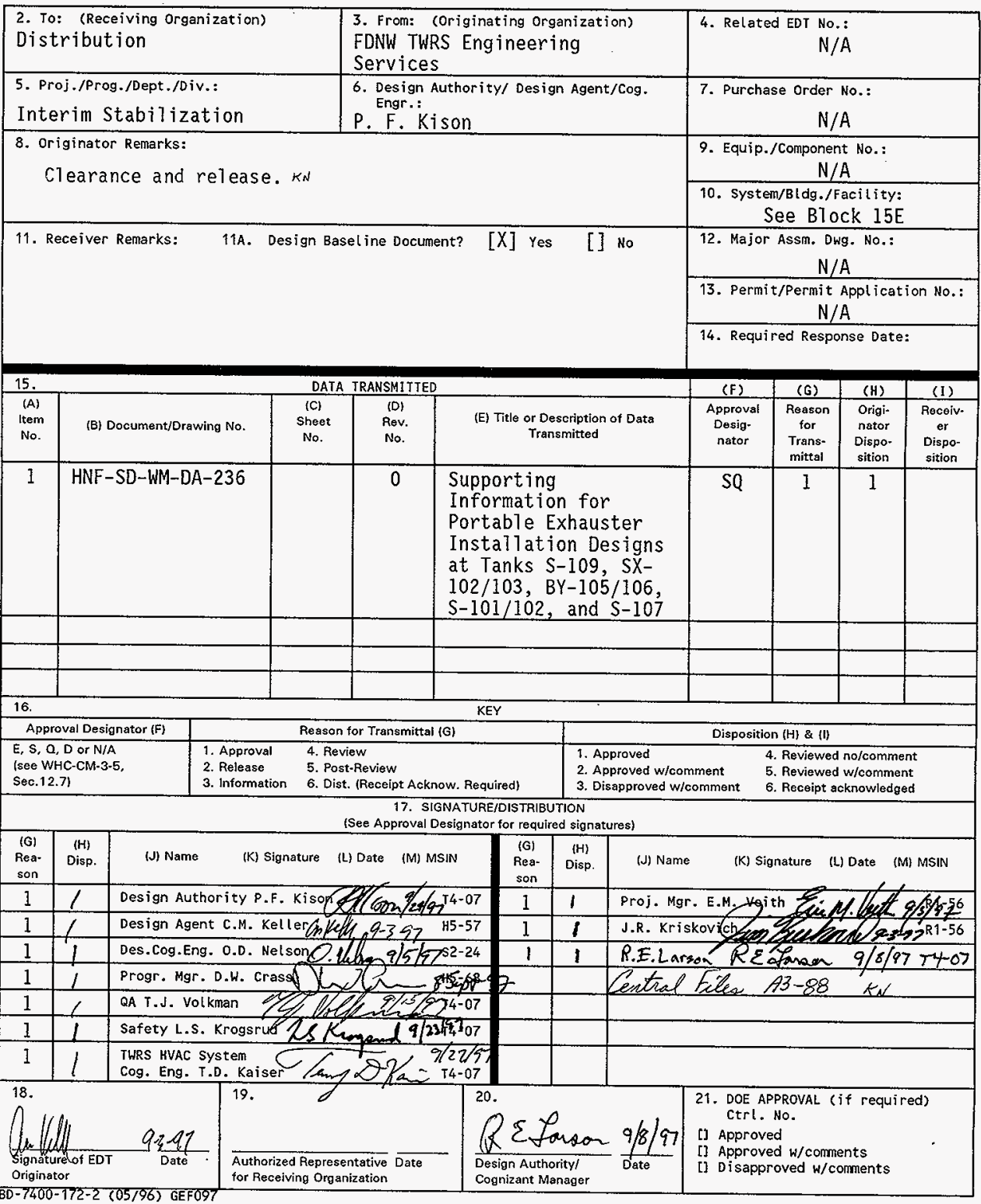




\section{SUPPORTING DESIGN INFORMATION FOR PORTABLE EXHAUSTER INSTALLATION AT TANKS S-109, SX-102/103, BY-105/106, S- 101/102, AND S-107}

Craig M. Keller

Fluor-Daniel Northwest, Richland, WA 99352

U.S. Department of Energy Contract DE-AC05-96RL13200

KN

EDT/ECN: 16145Z4 UC: 2030

Org Code: 04E00 Charge Code: C13697

B\&R Code: EW3120072 Total Pages: 202

Key Words: Portable Exhauster, Saltwe11 Pumping, Waste Tank Ventilation, Flammable Gas Mitigation

Abstract: This document provides supporting calculations and equipment dedication plans for portable exhausters and ductwork installed on tanks S-109, SX-102/103, BY-105/106, S-101/102, and S-107. The exhausters will ventilate the tanks during saltwell pumping to prevent the potential accumulation of flammable gases.

IRADEMARK DISCLAIMER. Reference herein to any specific commercial product, process, or service by trade name, tradenark, manufacturer, or otherwise, does not necessarily constitute or imply its endorsement, recomendation, or favoring by the United States Governuent or any agency thereof or its contractors or subcontractors.

Printed in the United States of America. To obtain copies of this document, contact: Document Control Services, P.O. Box 950, Mailstop H6-08, Richland WA 99352, Phone (509) 372-2420; Fax (509) 376-4989.
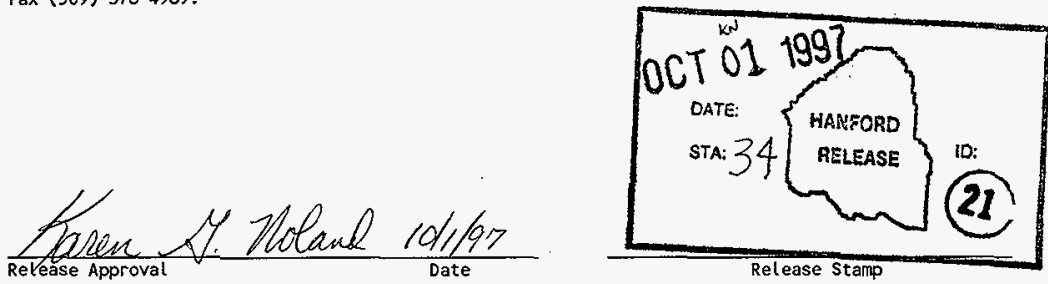


\section{SUPPORTING INFORMATION FOR PORTABLE EXHAUSTER INSTALLATION DESIGNS \\ AT TANKS S-109, SX-102/103, BY-105/106, S-101/102, AND S-107}

Prepared by,

Craig N. Keller

Fluor Daniel Northwest

Richland, WA 


\section{TABLE OF CONTENTS}

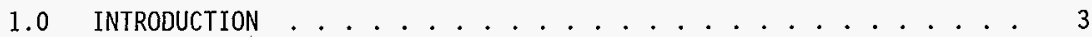

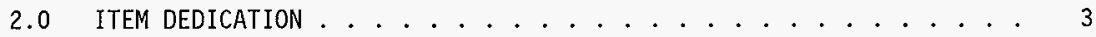

3.0 DESIGN CALCULATIONS ...................... 6 APPENDIX A: DESIGN CALCULATIONS FOR TANK S-109 INSTALLATION . . . . A-1 APPENDIX B: DESIGN CALCULATIONS FOR TANKS SX-102/103 INSTALLATION . . . B-1 APPENDIX C: DESIGN CALCULATIONS FOR TANKS BY-105/106 INSTALLATION . . . C-1 APPENDIX D: DESIGN CALCULATIONS FOR TANK S-107 INSTALLATION . . . . . D-1 APPENDIX E: DESIGN CALCULATIONS FOR TANKS S-101/102 INSTALLATION . . . E-1 APPENDIX F: JUSTIFICATION FOR ELECTRICAL SUPPLY AND DISTRIBUTION . . . . F-1 
HNF-SD-WM-DA-236

Rev. 0

\subsection{INTRODUCTION}

This document contains design calculations and equipment dedication $\mathrm{pl}$ ans to support the installation designs of portable exhausters and ductwork on waste tanks S-109, SX-102/103, BY-105/106, S-101/102, and S-107. This document provides information to ensure that the designs meet appiicable design criteria at the time they were prepared. The exhausters will ventilate the tanks during saltwell pumping to prevent the potential accumulation of flammable gases. Some of the exhausters will be used to ventilate a single tank and others will ventilate two tanks. Installation of the exhausters is planned for FY-98. The following Engineering Change Notices (ECN) provide the exhauster installation design for the tanks.

ECN 618790: $500 \mathrm{cfm}$ exhauster on tank S-109

ECN 618792: $500 \mathrm{cfm}$ exhauster on tanks SX-102 and SX-103

ECN 61879.3: $500 \mathrm{cfm}$ exhauster on tanks BY-105 and BY-106

ECN 618794: $1000 \mathrm{cfm}$ exhauster on tank S-107

ECN 618795: $1000 \mathrm{cfm}$ exhauster on tanks S-101 and S-102

Dome loading due to the exhauster, ductwork, and installation activities will be considered prior to the installations. Dome loading due to the weight of the exhauster (9500 lbs) and ductwork (2000 1bs est.) is not expected to be a problem based on the allowable load of 200,000 $7 \mathrm{~b}$ for single shell tanks and considering other equipment loads. Dome loading due to the installation activities is also not expected to be a problem assuming the loadings will be similar to the portable exhauster installation on tank A-101.

A field walkdown should be performed prior to exhauster installation due to the significant time span between preparation of the designs and the installation. The walkdown could be used to verify availability of space and electrical power required for the installation. The appropriate ECN's should be reviewed to determine required space and electrical requirements prior to the walkdown.

2.0 ITEM DEDICATION

A few items required for the exhauster installation are classified as safety significant/safety class 2 and were procured as commercial grade items. It is required that these items undergo a dedication process to verify their critical safety characteristics. Table 1 provides a compilation of the items requiring dedication, their critical function, and their method of dedication. The majority of items will be dedicated by performing a pressure/leak test in accordance with ASME B31.3 as part of the exhauster installation. 


\section{TABLE 1: DEDICATION OF ITEMS FOR EXHAUSTER INSTALLATION}

\begin{tabular}{|c|c|c|c|c|c|}
\hline ITEM & SAFETY FUNCTION & FAILURE MODE & FAILURE EFFECT & $\begin{array}{c}\text { SAFETY } \\
\text { CLASSIFICATION }\end{array}$ & DEDICATION METHOD \\
\hline $\begin{array}{l}\text { Expansion Joints for } \\
\text { Ventilation Plenum }\end{array}$ & $\begin{array}{l}\text { Provide containment of } \\
\text { potentially contaminated } \\
\text { airstream } \\
\text { Provide flexibility for } \\
\text { plenum movements due to } \\
\text { seismic/thermal effects }\end{array}$ & $\begin{array}{l}\text { Externat or internal } \\
\text { event causes structural } \\
\text { failure of the } \\
\text { components }\end{array}$ & $\begin{array}{l}\text { Failure could lead to an } \\
\text { unfiltered release of tank } \\
\text { vapors }\end{array}$ & $\begin{array}{l}\text { Safety Significant/SC-2 } \\
\text { MRP } 4-46 \text { Table 1, Item } \\
12\end{array}$ & $\begin{array}{l}\text { I tems purchased as SC-2 } \\
\text { commercial Grade I tem. } \\
\text { Perform pressure/leak test } \\
\text { in accordance with ASME } \\
\text { B } 1.3 \\
\text { Allowable movements for } \\
\text { expansion joints provide a } \\
\text { significant (>3) margin of } \\
\text { safety for intended } \\
\text { application. }\end{array}$ \\
\hline $\begin{array}{l}\text { Pipe support slide } \\
\text { for ventilation } \\
\text { plenum }\end{array}$ & $\begin{array}{l}\text { Provide attachment for } \\
\text { structural support of the } \\
\text { ventilation plenum }\end{array}$ & $\begin{array}{l}\text { External or internal } \\
\text { event causes structural } \\
\text { faiture of the } \\
\text { components } \\
\text { Weld attachment of } \\
\text { support slide results } \\
\text { in material } \\
\text { incompatibility with } \\
\text { the ventilation plenum. }\end{array}$ & $\begin{array}{l}\text { Failure could lead to an } \\
\text { unfiltered release of tank } \\
\text { vapors due to breach of } \\
\text { ventilation plenum } \\
\text { containment. }\end{array}$ & $\begin{array}{l}\text { Safety Significant/SC-2 } \\
\text { MRP } 4-46 \text { Table 1, I tem } \\
15\end{array}$ & $\begin{array}{l}\text { Items purchased as } 5 C-2 \\
\text { Commercial Grade Item. } \\
\text { Perform Eddy current } \\
\text { evaluation to insure } \\
\text { material is } 300 \text { series sST } \\
\text { hhich is compatible for } \\
\text { welding to ventilation } \\
\text { plenum. } \\
\text { Allowable load rating for } \\
\text { pipe slide provides a } \\
\text { significant (>3) margin of } \\
\text { safety for intended } \\
\text { application. }\end{array}$ \\
\hline $\begin{array}{l}\text { Isolation valves for } \\
\text { ventilation plenum } \\
\text { and condensate } \\
\text { piping }\end{array}$ & $\begin{array}{l}\text { Provide containment of } \\
\text { potentially contaminated } \\
\text { airstream and condensate } \\
\text { liquids. }\end{array}$ & $\begin{array}{l}\text { External or internal } \\
\text { event causes structural } \\
\text { or operational failure } \\
\text { of the components. }\end{array}$ & $\begin{array}{l}\text { Failure could result in a } \\
\text { release of tank vapors or } \\
\text { condensate liquid }\end{array}$ & $\begin{array}{l}\text { Safety significant/SC-2 } \\
\text { MRP } 4-46 \text { Table 1, I tem } \\
12 \\
.\end{array}$ & $\begin{array}{l}\text { Items purchased as SC-2 } \\
\text { Commercial Grade Itern. } \\
\text { Perform pressure/leak test } \\
\text { in accordance with ASME } \\
\text { B31.3 } \\
\text { Pressure rating of valves } \\
\text { provide a significant ( }>3 \text { ) } \\
\text { margin of safety for } \\
\text { intended application }\end{array}$ \\
\hline
\end{tabular}


HNF-SD-WM-DA-236

Rev. 0

\begin{tabular}{|c|c|c|c|c|c|}
\hline ITEM & SAFETY FUNCTION & FAILURE MODE & FAILURE EFFECT & $\begin{array}{c}\text { SAFETY } \\
\text { CLASSIFICATION } \\
\end{array}$ & DEDICATION METHOD \\
\hline $\begin{array}{l}\text { Gasket Material for } \\
\text { ventilation plenum } \\
\text { and condensate } \\
\text { piping }\end{array}$ & $\begin{array}{l}\text { Provide containment of } \\
\text { potentially contaminated } \\
\text { airstream and condensate } \\
\text { liquids }\end{array}$ & $\begin{array}{l}\text { Gasket material fails } \\
\text { to provide containment } \\
\text { at flanged joints. }\end{array}$ & $\begin{array}{l}\text { Fajlure could result in a } \\
\text { release of tank vapors or } \\
\text { condensate liquid }\end{array}$ & $\begin{array}{l}\text { Safety Significant/Sc-2 } \\
\text { MRP 4-46 Table 1, Item }\end{array}$ & $\begin{array}{l}\text { I tens purchased as SC-2 } \\
\text { Commercial Grade I tem. } \\
\text { Perform pressure/leak test } \\
\text { in accordance wi th ASME } \\
B 31.3\end{array}$ \\
\hline
\end{tabular}


HNF-SD-WM-DA-236

Rev. 0

\subsection{DESIGN CALCULATIONS}

Calculations to verify adequacy of the installation designs were prepared and checked. A copy of the calculations for each installation are provided in Appendices $A$ through $E$ of this document. Calculations determined static pressure losses in the ductwork and analyzed for seismic, wind, thermal, and missile effects. Ventilation ductwork was analyzed using criteria in the ASME B31.3 Process Piping Code. Analysis determined all the designs to be acceptable. Justification for the electrical supply and distribution to the portable exhausters is provided in Appendix F. 
HNF-SD-WM-DA-236

Rev. 0

APPENDIX A: DESIGN CALCULATIONS FOR TANK S-109 INSTALLATION 
Fluor Daniel Northwest, Inc.

CALCULATION IDENTIFICATION AND

INDEX
Page 1 of 21

Date

$1 / 17 / 97$

This sheet shows the status and description of the attached Design Analysis sheets.

Discipline Mechanical

wo/job No. E40690

Calculation No. M-1

Project No. \& Name S-109 500 CFM Exhauster Installation

Calculation Item

These calculations apply to:

Dug. No. ECN 618790

Rev. No.

Dwg. No.

Rev. No.

other (Study, CDR)

Rev. No.

The status of these calculations is:

[] Pretiminary Calculations

[X] Final Calculations

[] Check Calculations (On Calculation Dated)

[] Void Calculation (Reason voided )

Incorporated in Final Drawings? [] Yes [X] No

This calculation verified by independent "check" calculations? [] Yes [] No

Original and Revised Calculation Approvals:

\begin{tabular}{|l|c|c|c|}
\hline & $\begin{array}{c}\text { Rev. } 0 \\
\text { Signature/Date }\end{array}$ & $\begin{array}{c}\text { Rev. 1 } \\
\text { Signature/Date }\end{array}$ & $\begin{array}{c}\text { Rev. 2 } \\
\text { Signature/Date }\end{array}$ \\
\hline Originator & & & \\
\hline Checked by & & & \\
\hline Approved by & & & \\
\hline $\begin{array}{l}\text { Checked Against } \\
\text { Approved vendor Dat }\end{array}$
\end{tabular}

Design Analysis
Page No.

\begin{tabular}{|l|l|}
\hline 3 & Analysis of HEPA filter installation \\
\hline 7 & Analysis of ductwork \\
\hline 12 & Analysis of ductwork support \\
\hline $14 / 6$ & Ventilation pressure losses \\
\hline & \\
\hline & Attachments \\
\hline & \\
\hline & \\
\hline & \\
\hline & \\
\hline
\end{tabular}


Fluor Daniel Northwest, Inc.

\section{DESIGN ANALYSIS}

Client LMHC

subject S-109 Exhauster Installation

Location 241-S-109
Calc. No. M-1

Revision 0

Page No. 2 of 21

No/Job No. E40690

Date

checked

Revised
By C. M. Keller

By F. K.meterson

By

\section{OBJECTIVE:}

Perform analysis on S-109 exhauster and HEPA filter installation to verify stresses are within acceptable values and to determine expected pressure drop in the ventilation ducting.

\section{CRITERIA:}

Ventilation ducting and condensate piping are considered safety significant/safety class 2. The waste tank including risers are considered safety class 1. Design loads are specified in GC-Load -01. Allowable piping stresses are specified in the ASME B31.3 Process Piping Code.

\section{ASSUMPTIONS/GIVENS:}

Similarity between the S-109 exhauster installation and that performed on tank A101 allows partial application of previousiy performed analysis to this case.

CALCULATIÖN METHOD: Hand calculations are used.

\section{REFERENCES:}

1) Design Loads for facilities, GC-LOAD-01, Rev 1, ICF Kaiser Hanford Company

2) Structural Analysis of Exhauster Skid, Calculation P4K700-M-001 Rev 0, Job \#C05205/P4K700, J. A. Tuck, May 20, 1996

3) Installation Piping Analysis, Job \#P4H514, Calculation M-1 Rev 1, B. M. Koons, $4 / 30 / 96$

4) Marks Standard Handbook for Mechanical Engineers, 9th edition, McGraw-Hill

5) 1993 ASHRAE Handbook Fundamentals, American Society of Heating, Refrigeration and Air-Conditioning Engineers, Inc.

6) Minimum Design Loads for Buildings and 0ther Structures ASCE 7-88, American Society of Civi] Engineers.

7) Mechanical Engineering Design, 4th edition, Shigley and Mitchell, McGraw-Hill CALCULATIONS:

Components attached to the tank risers have been analyzed using conservative SC 1 criteria. Those components are the inlet HEPA filter and the ventilation tee.

Remainder of the ventilation ducting has been analyzed using SC 2 criteria. Analysis of the condensate piping has not been performed due to similarity with the A-101 piping which was previously analyzed in reference 3 . 


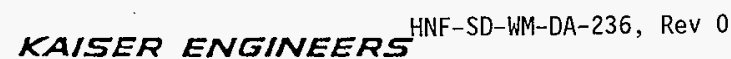
HANFORD

Client

Subject

Location

\section{DESIGN ANALYSIS}

Checked

Revised

\section{Page A-4}

Calc. No. $M-1$

Rovision 0

Page No. 3 of 21

WO/Job No. E40690

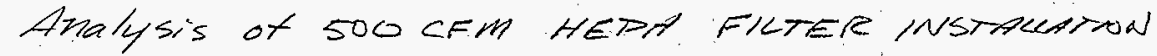
$\operatorname{Tank} 5-10912^{\prime \prime}$ RISER 6

BY CRAIC KELLER

BY ANN WETLNER

By

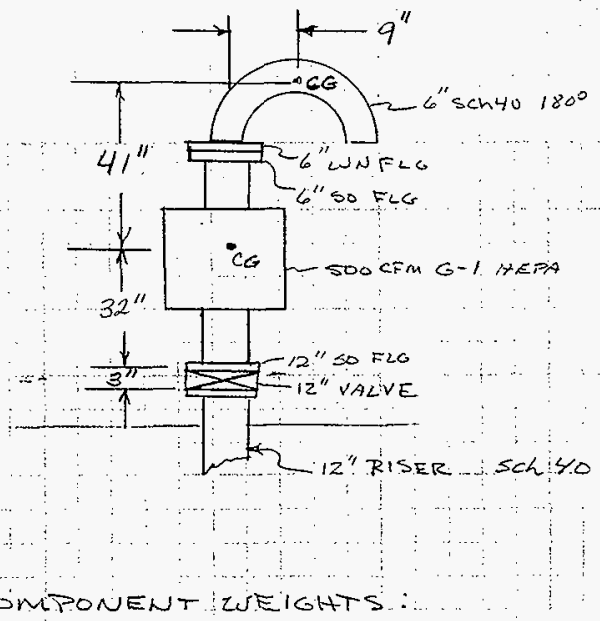

COMPONENT ZUEIGHTS:

$12 "$ VALVE 160 pel Vandor

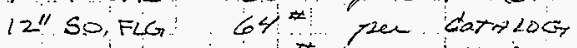
HEPA HLTER $230^{*}$ per Vendar

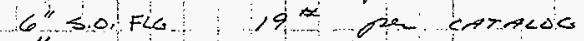
$6 " W N F L$ 25 Per CATMLOLT $6 " 180^{\circ} R=1,18^{*}$ pa chrol 546 rora

ANALSEL STRESS ON RISER DUE TO:O

1) SEMSiniC $\angle O A D I N C$

2) Wind $\angle \Delta A D \operatorname{Sic}$

3) $A 5 A$ COADNC

4) DERD LOADS

5). SNOW LOADING 


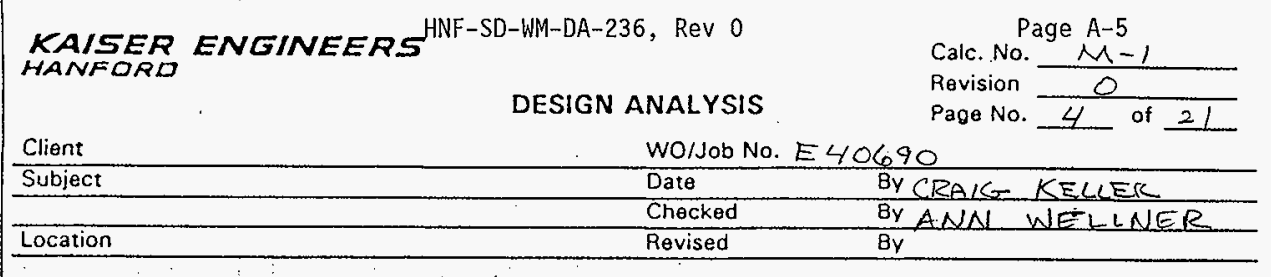

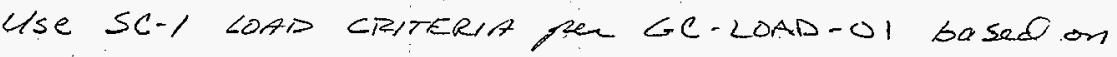
TONK RISER BEINC SCI.

Seínic Response UILL TSE ANALYZED STATICACLY USINA THE FEAK RCCELERATION OF THE RESPONSE SPECTRUM WIT S\%O CTITICA DMMPING, HOR SCI THE PEAK HORIZONTML PCCELERATION IS -W2g, and VERTCAL ACLELERATIONIS $2 / 3$ HORIZONTIF< . $28 \mathrm{~g}$

MAX VERTICAL LOAD ON RISER DUE TO SEISMIC EFFECT $\because S$

$$
(1289)\left(546 * 69^{*}\right.
$$

DETERMINE EENOINGMOMENT ON RISERTUE TO HORIZONTOL SEISMK LORID AND RETURN WNET.

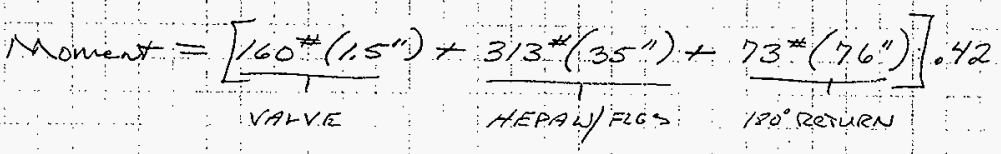

$$
\begin{aligned}
& +48\left(9^{\prime \prime}\right)=7464 \text { in } 16 \\
& \text { a } \quad x=120 \\
& c=\frac{1275}{2}=6.375 \\
& F=\pi / c 4\left(x_{0}^{4}-D_{2} 4\right)=279 \text { in } 4 \\
& \angle=\frac{M c}{I} \div \frac{7464(6.375)}{279}=1>0 \text { pi } 0<
\end{aligned}
$$

54-4300-037 KEH-0037.00 (06/92) 
KAISER ENGINEERS $S^{\text {HNF-SD-WM-DA-236, Rev } 0}$

DESIGN ANALYSIS

Page $A-6$

Calc. No. $M-1$

Revision 0

Page No. 5 of 21

Client WO/Job No. E40690

Subject Date BY CRAM KElLER

Location Checked BY ANN WEELNER

Revised

BY

HORIZONTAL LOADING DUE TO WIND PER ASCE7-88 (n fl) USING:

WIND SPEED $90 \mathrm{mph}$

Importance Factor 100

Exposure Class C

EXPOSED AREA: FILTER

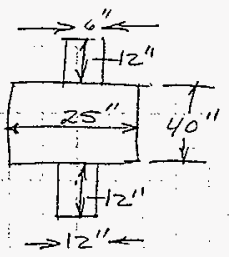

Add 20\% to account for valve and

$$
A=\left(6 \times 11^{2}\right)+(12 \times 12)+(25 \times 40)=1216 \mathrm{im}^{2}
$$

Return Anting.

$$
\text { torn Area }=1 / 2(1216)=1460 \text { in }
$$

WIND LOAD $F=q_{z} G_{h} C_{f} A_{f}$

ref 6

where,

$$
\begin{aligned}
& q_{z}=\text { Velocity Pressure } \\
& q_{z}=100256 \mathrm{Kz}(I X)^{2}
\end{aligned}
$$

$K_{z}=$ Velocity Pressure Exposure Coefficient $K z=0.80$. Table 6

$F=$ importance Factor $=1 \%$

$V=$ WIND SPEED $\mathrm{mph}=90$

$G_{n}=$ Gust Response Factor $=1.32$ Table 8

$C_{f}=$ Force Coefficient $=1,2$ : Table 13

$A_{f}=$ Projected Area $f t^{2}=\frac{1460}{144}=10 \mathrm{ft}^{2}$

$$
\begin{aligned}
& q z=.00256(180)(1.0 .90)^{2}=16.6 \\
& F=16.6(1.32)(1.2)(10)=263^{\not t} .
\end{aligned}
$$

$54.4300-037$ KEH-0037.00 $(06 / 92)$ 


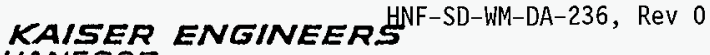 HANFORD}

DESIGN ANALYSIS
Page A-7

Calc. No. $M-1$

Revision $\frac{0}{6}$ of $\mathrm{Zl}$

Client

WO/Job No. E40690

Subject

Date

BYCRAIE KELLER

Location

Checked

BY ANN WELLNER

Revised

By

HORIZOMTAL FORCE DUE TO WINA LOADNNG IS ONLY MARGINALLY GREATER THEN HORIZONTL SEISMIC FORCE, THEREFORE WILL NOT FURTHER CALCULATE RISER STRESS DUE TO WUND LOAD,

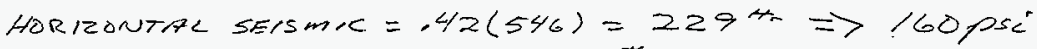
HORIZONTAL WIMP LOAD $=2630 K$

CONSIDER ASH/SNOW LOADING raf 1

ASH Cons: $24 \% / / F 2$

SNOWLOAD: ISF/F,

$\therefore$ ㅇ

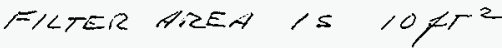

$$
F=240^{*} \text { dere to } 254
$$

MAX VERTICHL FORCE OW RISER IS $240^{\#-446 \%=786 \% ~ O . K . ~}$

Summary: Design of HEPA FILTER INSTPLCMTION IS ADEQUATE. No Aflditional Support is necded. 
KAISER ENGINEERS HF-SD-WM-DA-236, Rev 0

DESIGN ANALYSIS

Client

Subject

Location

Checked
Page A-8

Calc. No. $M-1$

Revision

Page No. 7 of

21

WO/Job No. $E 40690$

BY CRAM KELLER

By

ANALYSIS OE DUCTWORK FROM RISER TO EXHAUSTER.

ANaLYZE USINC SC2 LOADS FOR DuCTwark EVALUATE THE ROLCOWINC:

- LoAd on riser

- VerifY ALLOWABLE LIMITS fOR EXPANSION JOINT

- CHECK horizontal/VERtical loADS on ductwork

ANALYSIS:

Determine LOAD ON RISER-

$12^{\prime \prime}$ LAP JONI FLANGE $82^{\text {th }}$

$12^{\prime \prime} \operatorname{seh} 106^{\prime \prime} \mathrm{L}$

$12^{7 x}$

$1 Z^{\prime \prime} \times 6^{\prime}$ RED TEE

$75^{27}$

$12^{\prime \prime}$ WU FEC

$12^{\prime \prime}$ TICINO FCC

$80^{*}$

$6^{\prime \prime} \operatorname{seh} 4012^{\prime} \mathrm{L}$

$110^{*}$

C" BFLY VALVE

$10^{*}$

$\frac{40^{*}}{409^{*}}$

DRAT WEIGHT ON RISER AND EXPOSED AREA ARE LESS THEN FOR HERA FILTER INSTALLATION. NO FURTHER A ANALYSIS IS REQUIRED. RISER STRESS. IS.

LESS THAN DETERMINED FOR HERA FILTER INSTHLCATICN.

$54.4300-037 \quad \mathrm{KEH}-0037.00$ (06/92) 


$$
\begin{aligned}
& \text { KAISER ENGINEERS } \\
& \text { HANFORD }
\end{aligned}
$$

DESIGN ANALYSIS
Page A-9

Calc. No. $M-1$

Revision 0

Page No. 8 of 21

Client WO/Job No. E40690

Subject

Date

BY CRAIG KELLER

Location

Checked

BY ANN WELL NEE

Revised

By

VERIFY EXP. JOINT IS CAPABLE OF DISPLACEMENTS-

Seismic analysis to determine displacement of Exhauster SKID wAS previously performed for the. TANK $A-10$ installation in section $N$ of reference 2 .

Similarity between instrumatrows make the previous:.... results applicable to this instracarion. Horizontal displacement of the exhausted skiD wAs cakulared to be

. $083^{\prime \prime}$ for SC 2 seismic evert and 28 "for SC1 event.

EXP JOLT: PATHWAY NSF SOI2C-MI

ALLOWABLE AXIAL EXTENSION: ".O"

AXIAL COMPRESSION: L.99"

LATERAL MUMS: O.68"

- Check LATERAL mOVEMENT. USE CONSERURTNE

DISPLACEMENT OF SKID IN SCI EVENT.

$.28<.68 \quad 0 k$

- Check AXIAL MOVEMENT. COMBINE SKID SEISMIC DISPLACEMENT AND DUCT THERMAL EXPANSION.

Expansion Coefficient for $5 s$ is $9,6 \mathrm{E}-6 / \mathrm{F}^{\circ}$ rf 7

For $10^{\prime}$ Duct length wi $\Delta T=150^{\circ} \mathrm{F}$

$$
\Delta L=120^{\prime \prime}\left(150^{\circ}\right)(9,65-6)=.17 "
$$

TOT HL DISPLACEMENT, $17 \times .28=.45<1.0^{\prime \prime} O K$

$54-4300-037$ KEH-0037.00 (06/92) 
KAISER ENGINEER SNF-SD-WM-DA-236, Rev 0

DESIGN ANALYSIS
Page $\mathrm{A}-10$

Cali. No. $M-1$

Revision $\frac{0}{9}$ of 21

Client WO/Job No. E40690

Subject

Date

BY CRAIG KELLER

Checked BY ANN WELLNER

Location

Revised

By

ANALYZE 10' SPAN OF TUOTWORK BETWEEN SUPPORTS.

Consider vertical loading due to pipe weight, induction, snow LOAD, and seismic events. Consider horizontra loading due to cunt and seismic events. use $5 c z$ colDs

Pipe: $6 "$ sch 40 ss $\omega_{p}=19.15 \mathrm{H} / \mathrm{Fr}$

$$
\begin{aligned}
& 6.62500 \\
& 6.06570
\end{aligned}
$$

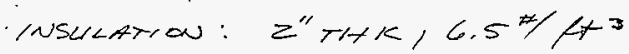

$$
\cdots \quad \omega_{z}=(6.54 / 43) \pi / 4\left(\left(\frac{10,625}{12}\right)^{2}-\left(\frac{0.625}{12}\right)^{2}\right)=2.4 \mathrm{H} / \mathrm{fz}^{2}
$$

SnOW LOAD: $15 \mathrm{H} / \mathrm{ft}^{2}$

$$
\omega_{s}=(15 t / 1+2)\left(\frac{10.625}{12}\right)=13 \cdot 3 * / f t
$$

Seismic forces cure BE ANALYZED by a simplified METHOD OF APPLYING THE FLAK ACCELERATION TAKEN FROM THE RESPONSE SPECTRUM PROVIDED IN REF. 1. THIS METHOD OF ANALYSIS IS JUSTIFIED BY THE SIMPLE CONFIGURATION TEEING ANALYZED. PEAK HORIZONTAL ACCELERATION IS. $26 \mathrm{~g}$ THEN FROM FIG. 5 in Ref 1. USINC 5\% CrITICAl DAMPINC, VERTICAL ACLELERATION is $2 / 3$ HORIZONTAL EQUATING TO $=17 \mathrm{~g}$.

VERTICAL SEISM FORCE: $(19,15+2.4,7 / \mathrm{Ft})(.17)=3.7 \% / \mathrm{Ft}$

$\therefore$ SHOW LOAD IS GREATER THAN VERTICAL SEISMIC FORCE.

54-4300-037 KEH-0037.00 $(06 / 92)$ 


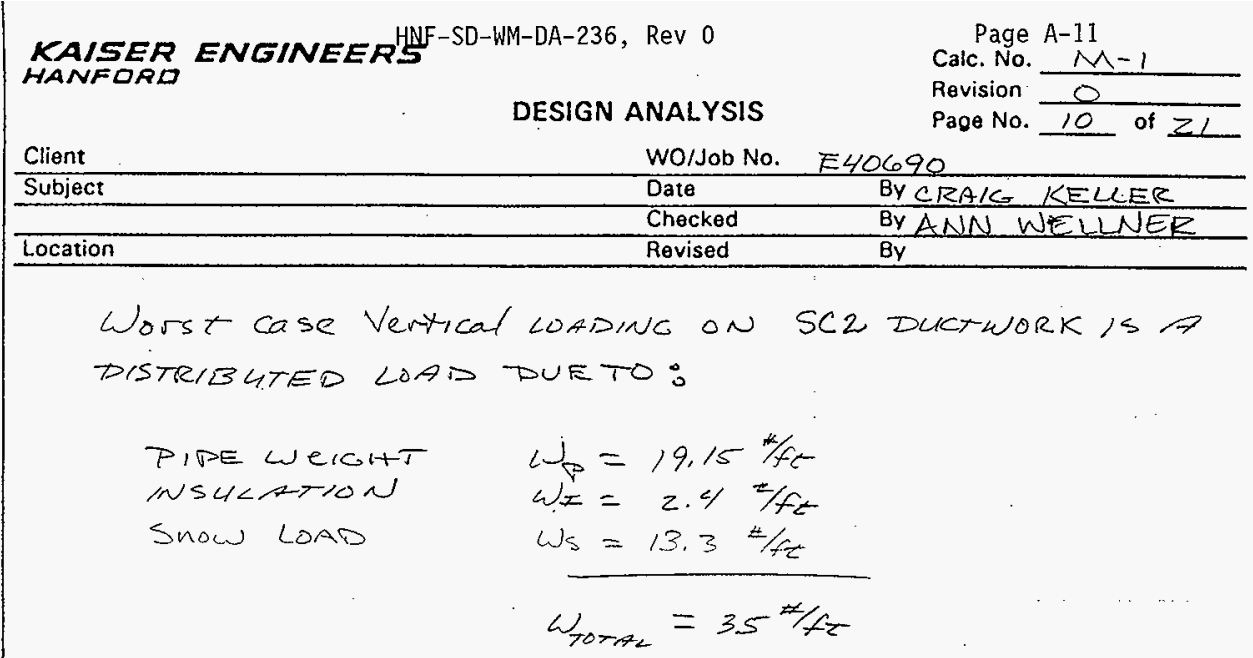

Analyze pipe as simply supported beam w/ uniform distributed load. Use pipe length of 10 '

$$
\begin{aligned}
& \dot{M}_{\text {max }}=\frac{\omega x}{2}(1-x) \quad \omega / x=l / 2 \quad \text { er. } 7 \\
& =\frac{\omega l}{4}(l-l / 2)=\frac{\omega l^{2}}{8}=\frac{(35 \mathrm{k} / \mathrm{ft})(10 \mathrm{ft})^{2}}{8}=437.5 \mathrm{ft} \cdot 16=5250 \mathrm{in} \cdot 16 \\
& I=\pi / 64\left(D_{0} 4 D_{i}{ }^{4}\right)=\pi / 64\left(6.625^{4}-6.065^{4}\right)=28 \mathrm{in}^{4} \\
& \sigma_{\text {max. }}=\frac{(5250 \mathrm{im}-16)(3.313 \mathrm{in})}{28 \mathrm{in}^{4}}=621 \mathrm{pssi}: 0 K
\end{aligned}
$$

Per B3/.3 ALCOWABLE STRESS IN DUCT PIPE is $16.7 \mathrm{KSL}$.

54-4300-037 KEH-0037.00 (06/92) 


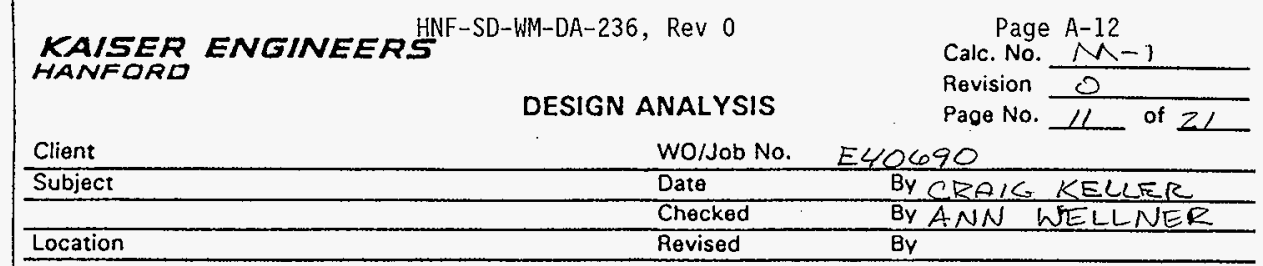

EVALUATE HORIZONTAL LOADS DUE TO WIND AND SEISMIC Seismic $=(19.15+24)\left(10^{\prime}\right)(.26)=56^{*}$

Wind: Use SC2 80 mph wind velocity

$$
\text { Area }=\left(10^{\prime}\right)\left(1 g^{\prime}\right)=8.3 f t^{2}
$$

wino $F=q_{2} G_{h} C_{f} A_{f}$ ref 6

$$
\begin{aligned}
& q_{2}=.00256 \mathrm{kz}(I V)^{2} \\
& k_{i}=.80 \\
& \text { TABLE } \\
& F^{\circ}=1.0 \\
& V=80 \mathrm{mph} \\
& g z=13.1 \\
& G_{n}=1.32 \text { TABLE } 8 \\
& C_{f}=1.2 \text { TABlE } 13 \\
& A=8.3 \\
& F=(13.1)(1.32)(1.2)(8.3)=172
\end{aligned}
$$

HORIZONTAL LOAD ON DUCTWORK IS SUPPORTED EQUALLY BY THE EXHAUSTER AND THE PIPE SUPPORT. PIPE SUPPORT LOAD IS $86^{\text {\#h }}$

$54-4300.037$ KEH-0037.00 (06/92) 


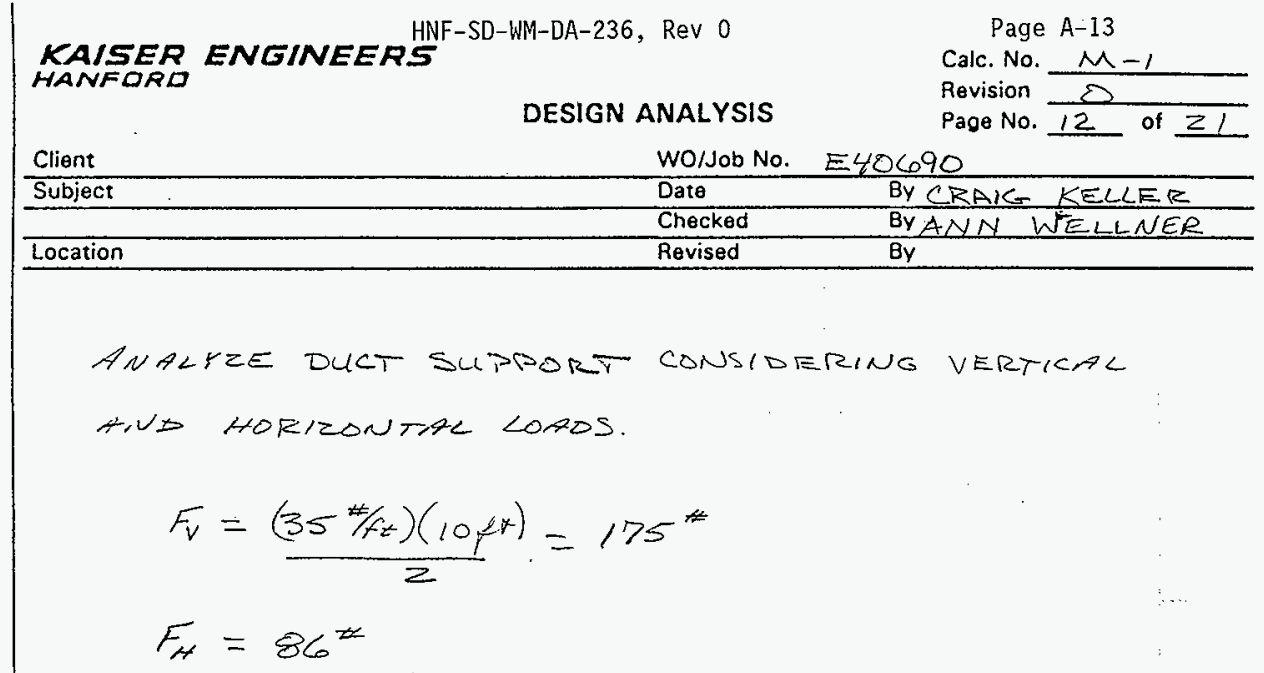

Dipe Support is GRINNELC FIG 280 Raxed Capacity 8,000 $4 "$ sch 40 pipe $45 \mathrm{~m}$ 453 48" Length $00=4.5^{\prime \prime} 20=4.03$ ".

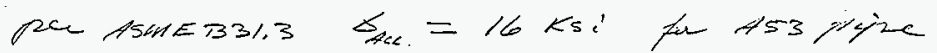

Concute footing $\begin{aligned} 30^{\prime \prime} S Q, 3000 \mathrm{psi} / & \rho=100 \% / \mathrm{H}^{3} \text { (conservative) } \\ 10^{\prime \prime} \mathrm{DP} & =521 \%\end{aligned}$ Support $\omega_{t} 50^{10 " D P}$

SO1K COAD $\frac{175^{k}+521^{*}+50^{*}}{(39 / 12)^{2}}=120$ psf OK

- Typical ALOWABLE SOIL LOAD AT HANFORD IS 2000 pSF.

EVAUIATE BENDINC STRESS INPITE

$$
\begin{aligned}
& x=\pi / 64\left(4,5^{4}-4.03^{4}\right)=2.18 \\
& \sigma=\frac{86\left(48^{\prime \prime}\right)(2.25)}{7.18}=1293 \text { psi } \text { OK }
\end{aligned}
$$

CHECK OVERTURMINC OF SUPFORT

$$
\omega t=(100 t / 1,3)(30 / 12)^{2}(1 / 12)=52, \#
$$

$54-4300.037$ KEH-0037.00 (06/92) 
KAISER ENGINEER HNF-SD-WM-DA-236, Rev 0 HANFORD

DESIGN ANALYSIS
Page A-14

Calc. No. $M-1$

Revision $\mathrm{O}$

Page No. 13 of 21
Client

Subject

Location

Location

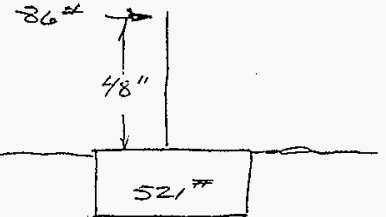

Overturnizg of support wikn wot occurn $<-30^{\prime \prime}->$

OVERTURN MOMENT $M_{0}=86.48=4 / 28$

RESTRAININC MOMENTT $M_{R}=521(15)=7815$

RasTRAING MOMENT IS CREATER THEN $O V \equiv R T U R N W N$,

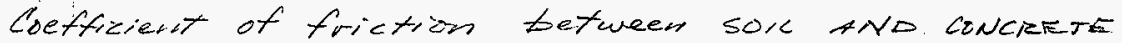
BASE IS CONSFRVATVELY ESTIMATES TO TEE . 4 S. SINCE $\therefore-$

friction coefficiant is greater HAEN HORIZONTHL SEISMIC ACLELERATION, SLIDING OF PIPE SUPPORT IS NOTA CONKRRN. 


\section{KAISER ENGINEERS HNF-SD-WM-DA-236, Rev 0 HANFORD}

\section{Client}

Subject

\section{DESIGN ANALYSIS}

Page A-15

Calc. No. $M-1$

Revision

Page No.

WO/Job No. EYOC9O

Date

BY CEAK KELLER

Checked

Revised
BY ANN WELLNER

By

EVALUATE STRESS AT EXHAUSTER INLET. INLET IS 12 "SCh lO pipe sUPPORTES BY $3 \times 2 \times 1 / 4$ CS. ANGLE AS SHOWN TBELOW.
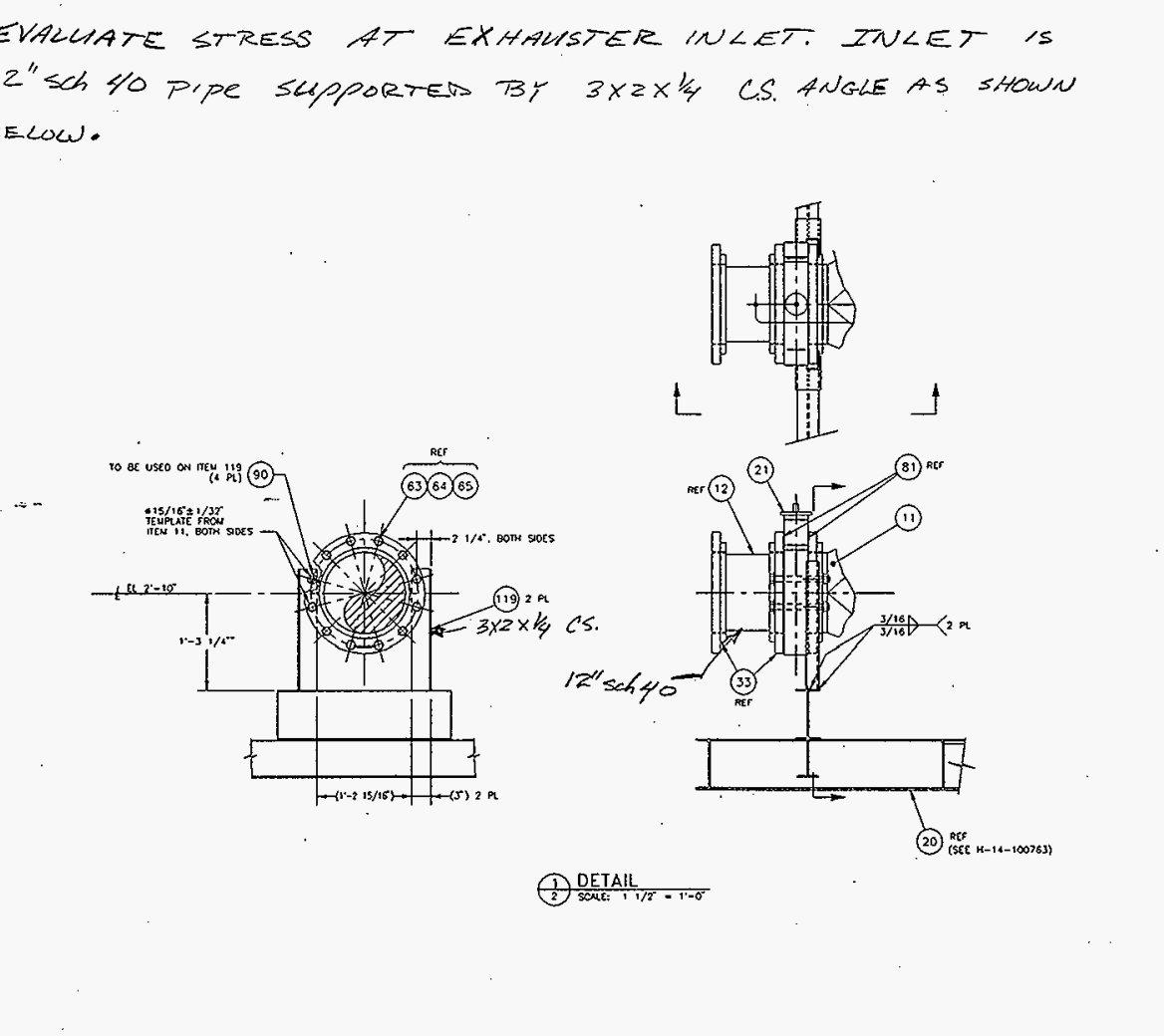

(1) DETALL

Verify. INCET SUP,PORT IS TOEQUATE FOR LOAPS IMPOSES PUE: TO SEISWIC AND TAERMAL FORCES. SUPPOHT IS "IA 36 CARBON STEEL WITH :

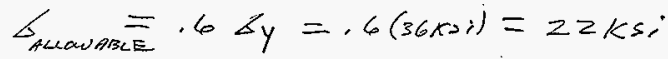

VerTICHC AND HORIONTAL LOADS ON THE WLET SUTPORT ARE NOT EVALUATED DUE TO THEIT SMALC MAGNITUDE.

$$
F_{V}=175^{*} \quad F_{H}=86^{*}
$$




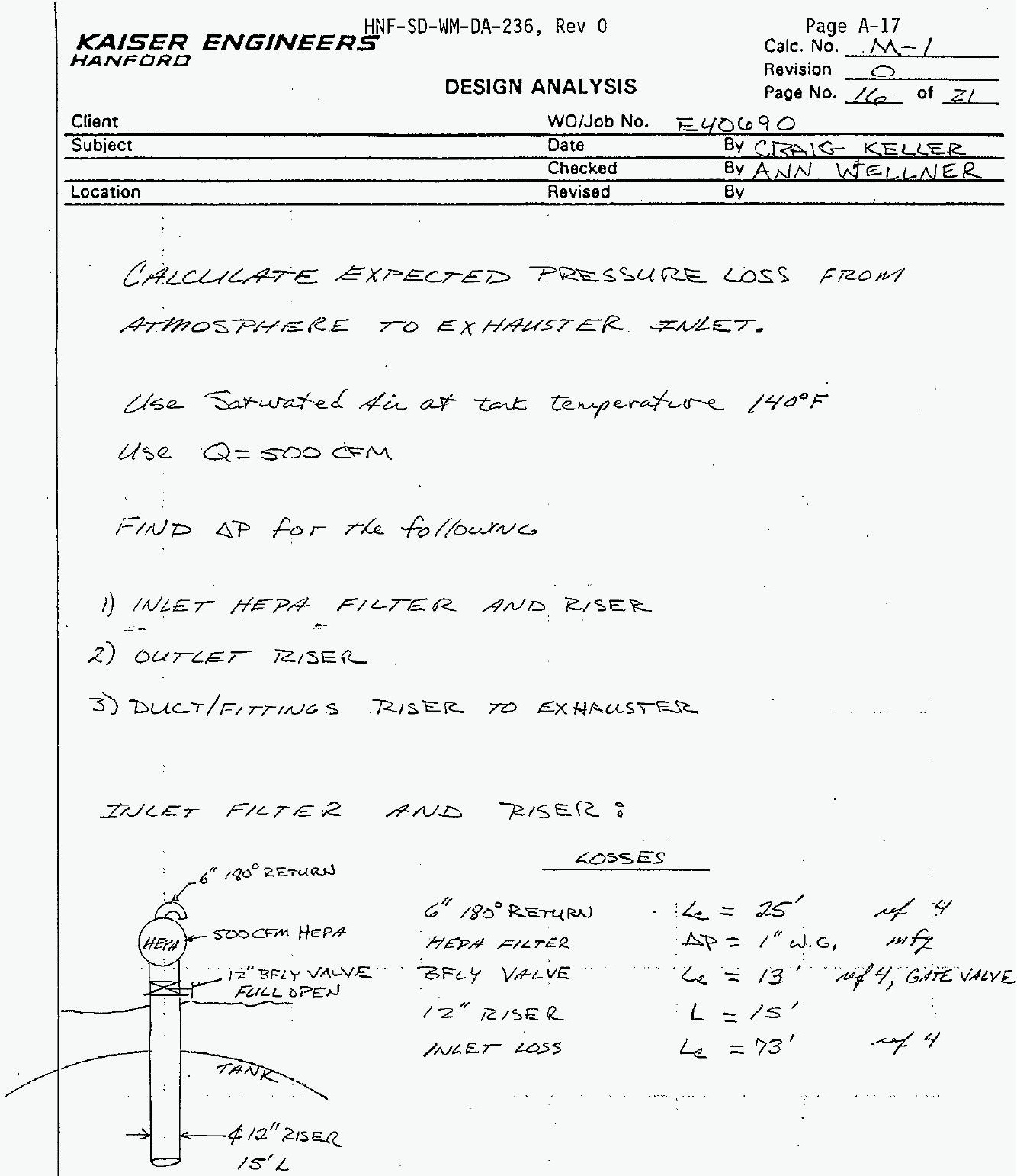


| HNF-SD-WM-DA-236, Rev 0 KAISER
HANFORD

Client

Subject

Location

OUTLET TISER:

\section{DESIGN ANALYSIS}

Page $A-18$

Calc. No. $M-1$

Revision

Page No.
WO/Job No. E40690

Date

Checked

Revised
BY CRAKC KEULER BY ANN WELNAER By

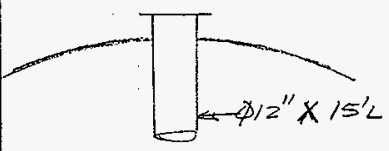

$$
\text { EX/T LOSS }
$$$$
\angle e=47^{\prime}
$$

RIDERPIPE

$L=15^{\prime}$

DUCT WORK:

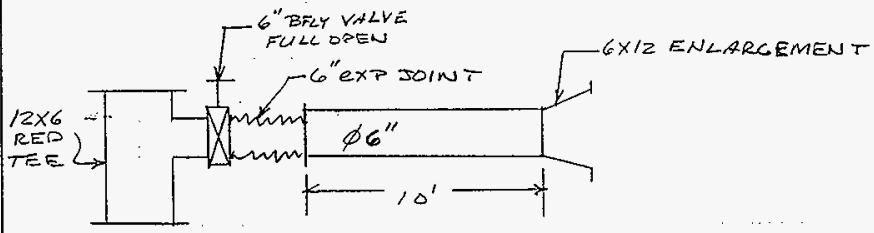

LOSS FOL SIDE OUTZET ONA IZX6 REDUCING TEE IS ASSUWIED EQUVALENT TO A IZ"SIDEOUTLET TEE FOLLOWEO BY A $12 \times 6$ CONTRACTION.

$$
\begin{aligned}
& 12^{\prime \prime} \text { SIDEOUTLET TEE Le }=60^{\prime} \text { wh } \\
& 12 \times 6 \text { CONTRACTION } L_{e}=11^{\prime} \text { ref } 4
\end{aligned}
$$

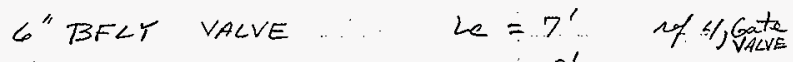

$$
\begin{aligned}
& \text { C"exp Joint } \quad L e=8^{\prime} \text { est. } \\
& 6^{\prime \prime} \times 10^{\prime} \text { DUKT } L=10^{\prime} \\
& \text { GXIZ ENCARGEMENT Le }=2 Z^{\prime} \text { eef }
\end{aligned}
$$




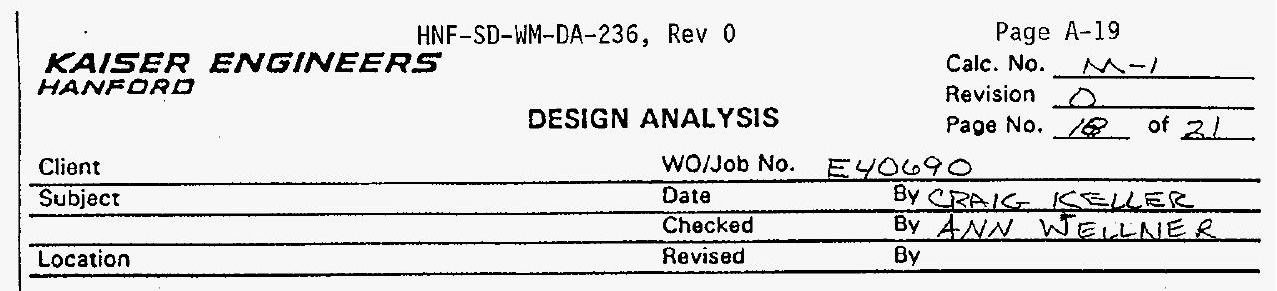

LOSS Summers:

$4^{\prime \prime}$ PIPE: $\quad 25^{\prime}+7^{\prime}+8^{\prime}+10^{\prime}+22^{\prime}=72^{\prime}$

$$
12^{\prime \prime} p_{1 p} \text { : } 13^{\prime}+15^{\prime}+73^{\prime}+47^{\prime}+15^{\prime}+60^{\prime}+1 \prime^{\prime}=234^{\prime}
$$

HEP FREER: $\triangle P=/ " W C$.

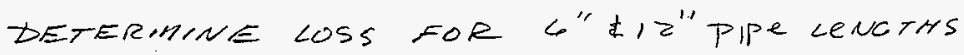

$$
\Delta p=f\left(\frac{12 L}{D}\right) \rho\left(\frac{V}{1097}\right)^{2} \text { we } 5 \rho j 32.4
$$

where: $\Delta p=$ static pressure coss (in W.C.)

$$
\begin{aligned}
& f=\text { friction factor } \\
& L=\text { Duct Length (ft) } \\
& D=\text { Duct DiAmetER (in) } \\
& v=\text { velocity (fem) } \\
& \rho=\text { Density }\left(16 \mathrm{~m} / \mathrm{fz}^{3}\right)
\end{aligned}
$$

Determine density for sari @ $140^{\circ} \mathrm{F}$

$$
\begin{aligned}
& p=(1 / v)(1+w) \\
& \text { eff } 5 \text { aq } 642 \\
& V=\frac{R_{a} T}{p-p \omega}=\text { moist ait specific volume } \\
& p=\text { rotc pressure }=14.7 \text { psi }=2117 \text { pst } \\
& P_{w}=\text { Tatial Pressure of water vapor }
\end{aligned}
$$

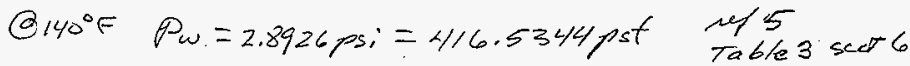

Table 3 set 6
KEH-0037.00 (06/92) 


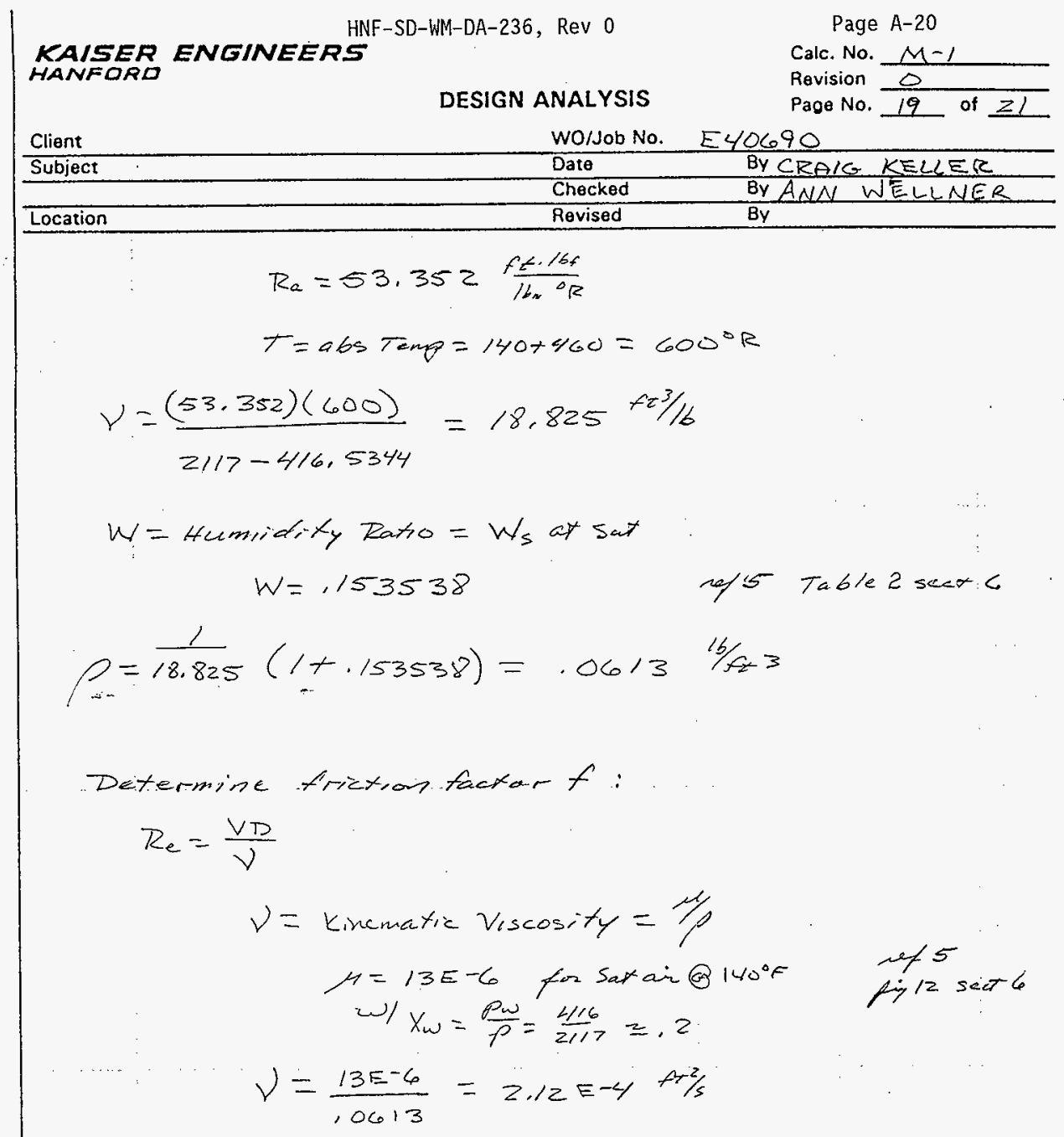

For soocfm in $\phi 6^{\prime \prime}$ sch 40 Duct

$$
\begin{gathered}
V=\frac{500}{\pi / 4\left(\frac{6.065}{12}\right)^{2}}=2492 \mathrm{fpm} \\
\operatorname{Re}=\frac{(2492 \mathrm{ft} / \mathrm{min})\left(\frac{6.065}{12} \mathrm{ft}\right)}{(2.12 \mathrm{E}-4 \mathrm{ft} / \mathrm{s})\left(\frac{60 \mathrm{sec}}{\mathrm{mm}}\right)}=9.9 \mathrm{EH} .
\end{gathered}
$$

Turbulent Flow

54-4300-037 KEH-0037.00 (06/92) 


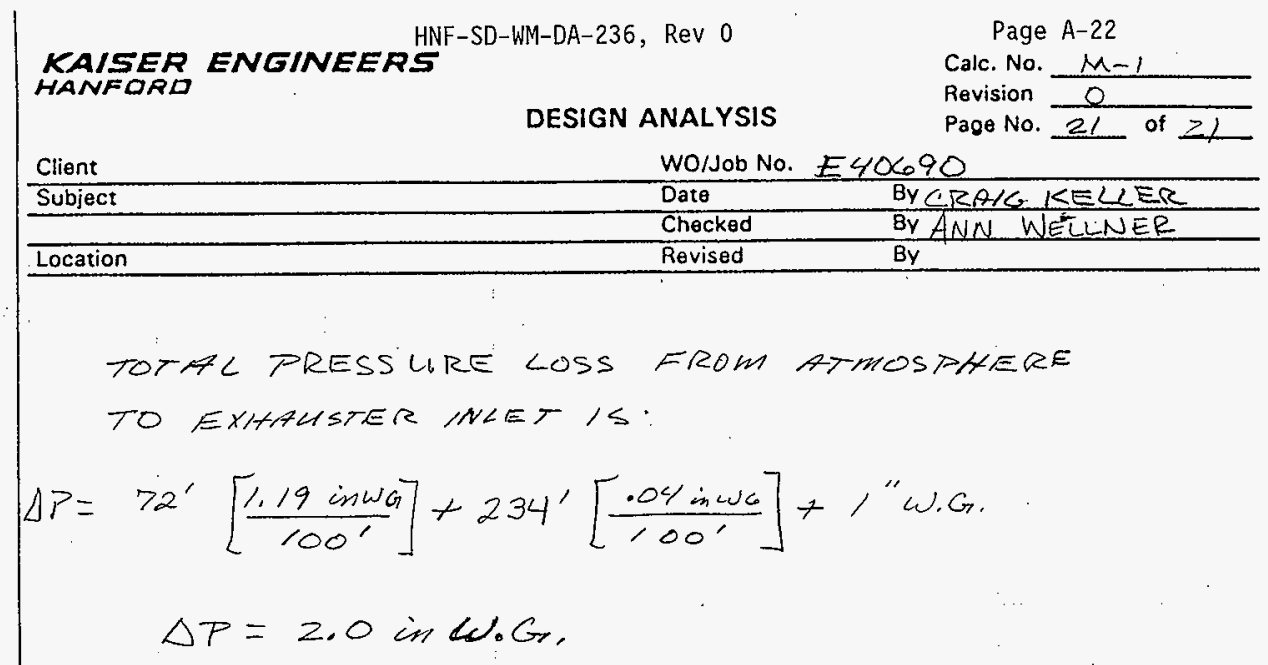


HNF-SD-WM-DA-236

Rev. 0

APPENDIX B: DESIGN CALCULATIONS FOR TANKS SX-102/103 INSTALLATION 
Fluor Daniel Northwest, Inc.

CALCULATION IDENTIFICATION AND INDEX

\begin{tabular}{|l|}
\hline Page 1 of $2 z$ \\
\hline $5 / 28 / 97$ \\
\hline
\end{tabular}

This sheet shows the status and description of the attached Design Analysis sheets.
Discipline Mechanical
No/Job No. E40690
Calculation No. $\mathrm{M}-2$

Project No. \& Name 241-SX-102/103 500 CFM Exhauster Installation

Calculation Item

These calculations apply to:

Dhg. No. ECN 618792

Rev. No.

Dwg. No.

Rev. No.

other (Study, COR)

Rev. No.

The status of these calculations is:

[] Preliminary Calculations

[X] Final Calculations

[] Check Calculations (On Calculation Dated )

[] Void Calculation (Reason voided )

$\begin{array}{lllll}\text { Incorporated in Final Drawings? } & {[]} & \text { Yes } & {[X]} & \text { No } \\ \text { This calculation verified by independent "check" calculations? } & {[]} & \text { Yes } & {[]} & \text { No }\end{array}$

Original and Revised Calculation Approvals:

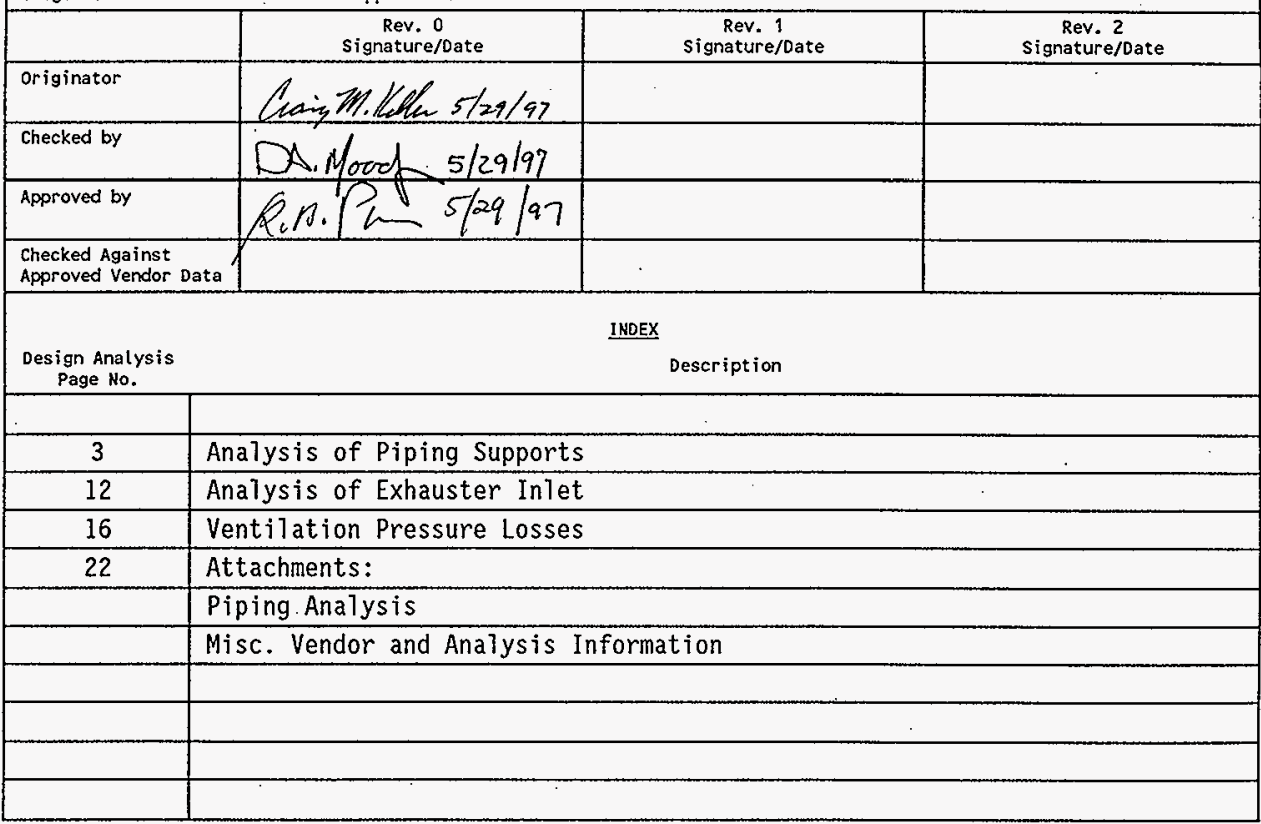


Fluor Daniel Northwest, Inc.

\section{DESIGN ANALYSIS}

client $\mathrm{LMHC}$

subject SX-102/103 Exhauster Installation

Location $241-S X-102 / 103$
Calc. No. $M-2$

Revision 0

Page No. 2 of 22

WO/Job No. E40690

Date $5 / 28 / 47$ By C. M. Keller

Checked $5 / 28 / 9>$ By D.A. Moos

Revised

By

\section{OBJECTIVE:}

Perform analysis on SX-102/103 exhauster installation (ECN 618792) to verify stresses are within acceptable values and to determine expected pressure drop in the ventilation ducting.

CRITERIA:

The exhauster and associated piping are analyzed using Safety Class 2 design loads provided in reference 1 . Piping is analyzed using criteria specified in the ASME B31.3 Process Piping Code. Structural members have been analyzed using criteria specifjed in reference 3 .

\section{ASSUMPTIONS/GIVENS:}

Piping stresses and support reactions are contained in the piping analysis provided in the Attachments section. Analysis of the exhauster condensate piping in refernce $\sigma$ is considered a governing case.

CALCULATION METHOD: Hand calculations and Autopipe analysis have been used to verify adequacy of the design.

\section{REFERENCES:}

1) Design Loads for facilities, GC-LOAD-01, Rev 1, ICF Kaiser Hanford Company

2) Minimum Design Loads for Buildings and Other Structures ASCE 7-88, A.S.C.E.

3) Manual of Steel Construction, Allowable Stress Design, 9th Edition, American Institute of Steel Construction, 1989

4) Marks Standard Handbook for Mechanical Engineers, 9th edition, McGraw-Hill

5) 1993 ASHRAE Handbook Fundamentals, American Society of Heating, Refrigeration and Air-Conditioning Engineers, Inc.

6) Installation Piping Analysis, Job \#P4H514, Calculation M-1 Rev 1, B. M. Koons, $4 / 30 / 96$

7) Mechanical Engineering Design, 4th edition, Shigley and Mitchell, McGraw-Hi1l

8) Design of Structures for Missile Impact, BC-TOP-9A Rev 2, Bechtel Power Corp.

9) Design of Expansion Anchors, GC-ANCR-01, Rev 1, ICF Kaiser Hanford Company

10) Roarks Formulas for Stress and Strain, 6th Edition, McGraw-Hi]l

11) Foundation Engineering Handbook, 2nd Edition, Hsai-Yang Fang 
HNF-SD-WM-DA-236, Rev 0

KAISER ENGINEERS

HANFORD

Client

Location

DESIGN ANALYSIS
Page $B-4$

Calc. No. $M-2$

Revision 0

Page No. 3 of 22

WO/Job No. E40690

Date $5 / 28 / 97$ By CN KELLER Checked $5 / 28 / 97$ By DA MOODY

ANALYZE STEESS ON VERTICAL PIPE SUPPORTS

SUPPORT DETALE:

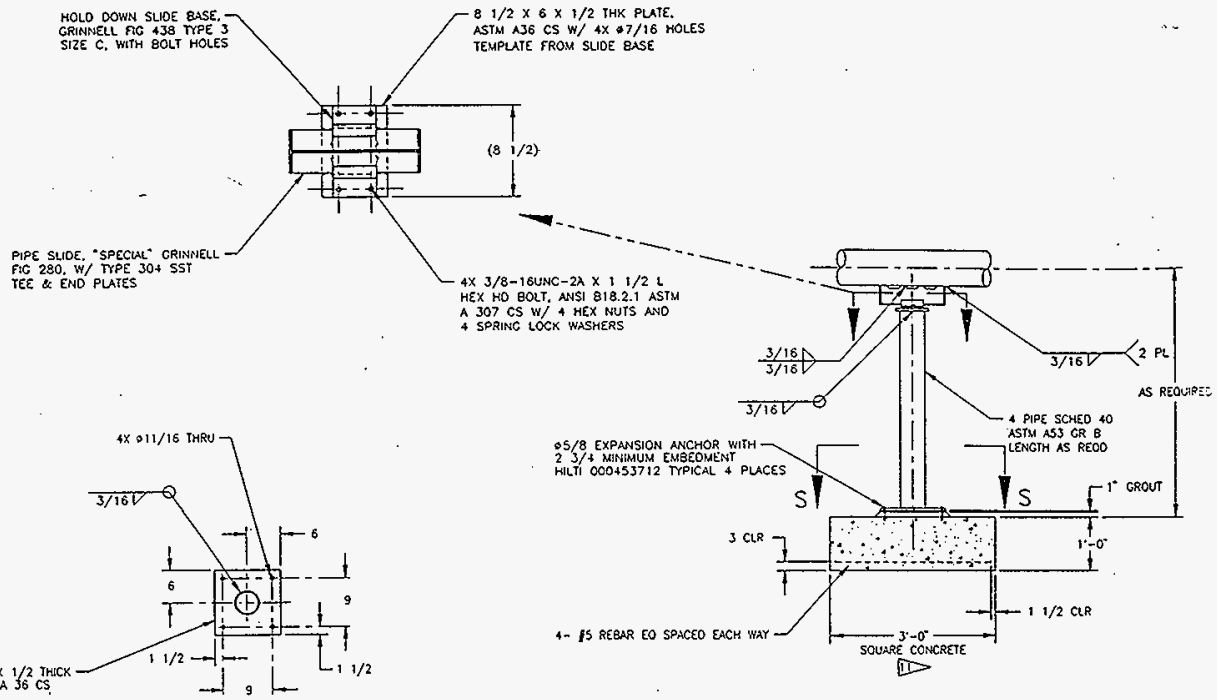

VIEW $G, 9$

SCALE: $1^{2}=\mathrm{t}^{\circ}-0^{\circ}$ (PAGES $7 * 8$ )

$\frac{\text { SECTION S-S }}{\text { SCAE: } r^{*}=1+1^{\circ}-\sigma^{\circ}}$

USF SUPPORT TEACTION FORCES FROM AUTOPIPE ANALYSIS.

WORST CASE HORIZONTAL LOADING IS SUPRORT BSO.

(NOTE: SUPPOTT BGS IS DIFFERNT TYPE ANALYZED LATER) 


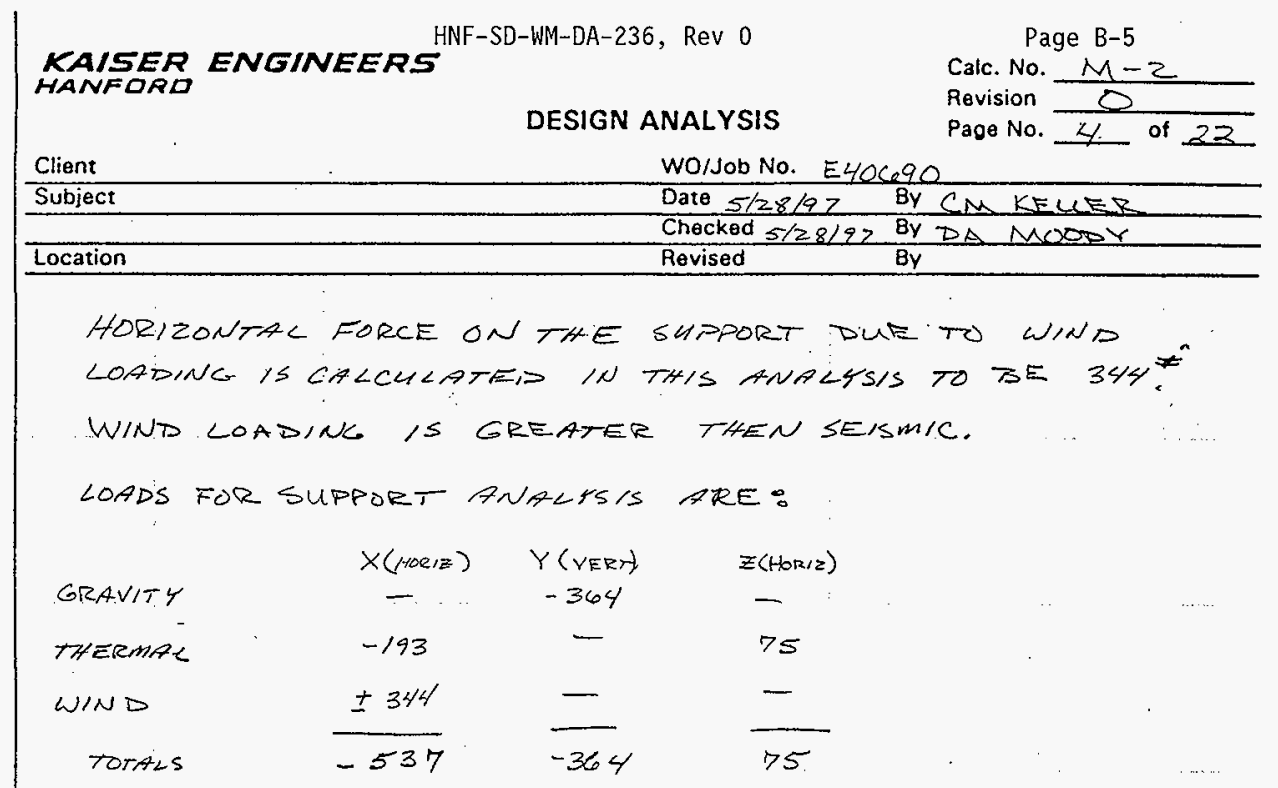

Determine support distance grade to pipe $q$ :

$G$ pipe elevation. 605.23'

Grate eleVation $661.71^{\prime}$ (AVG of 2 tankS)

Support Distance $(665,23-661,71)(12)=42 "$.

CHECK OVERTURNING OF SUPPORT:

OVERTURN MOMENT- $\leq$ Z/3 RESTORING MOMENT

$$
\Lambda_{0}=537^{*}\left(42^{\prime \prime}\right)=22554 \text { in } 16
$$

Concrete berisity $150 \% / \mathrm{ft}^{3}$

$$
\begin{aligned}
& M_{R}=\left[\left(9 f^{3}\right)\left(150^{H / t^{3}}\right)+364^{7}\right]\left(18^{\prime \prime}\right)=30852 \mathrm{in} 16 \\
& 2 / 3 M_{R}=20568 \mathrm{in} \cdot 16 \\
& 22554>20568 \text { NOT OK }
\end{aligned}
$$

$54-4300-037 \quad \mathrm{KEH}-0037.00(06 / 92)$ 


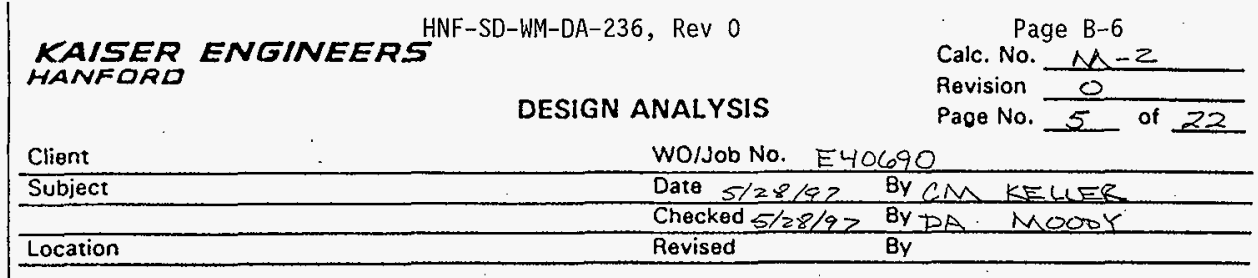

CHECK SUPPORT OVERTURN WITH CONCRETE BASE SIZE INCREASED TO 3'XY' WITH Y'PIMENSION PERPENDICULAR TO THE PIPE RUN.

$$
\begin{gathered}
M_{R}=[(12 f+3)(1507 / f \times 3)+364]\left(24^{\prime \prime}\right)=51936 \mathrm{in} 16 \\
2 / 3 M_{R}=34624 \text { in } 16 \\
22554<34624 \quad 0 K
\end{gathered}
$$

$\therefore$ SUpport Concrete base SIZE SHOULD toE NCREASED TO 3'X4'. THIS SIZE WILL PROVIDE THE MINIMUM 2/3 SAFETY MARGIN FOR SUPPORT OVERTURNING.

CHECK STRESS IN SUPPORT PIPE:

$y^{\prime \prime}$ sch 40 . ASTi 753 cS $\sigma_{y}=30$ Rsi men.

$$
\begin{aligned}
& S=3.21 \mathrm{in}^{3} \quad S_{M L L}=.6\left(\Delta_{y}\right)=18 \mathrm{ks} \cdot \\
& A=3.17 \mathrm{in}^{2} \\
& i=\frac{532}{3.17}=169 \mathrm{ps}
\end{aligned}
$$

Normal sties s DUE TO BENDING,

$$
\begin{aligned}
& M=\sqrt{537^{2}+25^{2}}\left(42^{\prime \prime}\right)=22,773 \mathrm{~m} / 4 \\
& \sigma=\frac{22773}{3.21}=2094 \mathrm{psi}<18 \mathrm{ksi} 0 \mathrm{~K}
\end{aligned}
$$

$\therefore$ SUPport pipe SIZE /S. ACLEPTPJLE.

54-4300-037 KEH-0037.00 (06/92) 


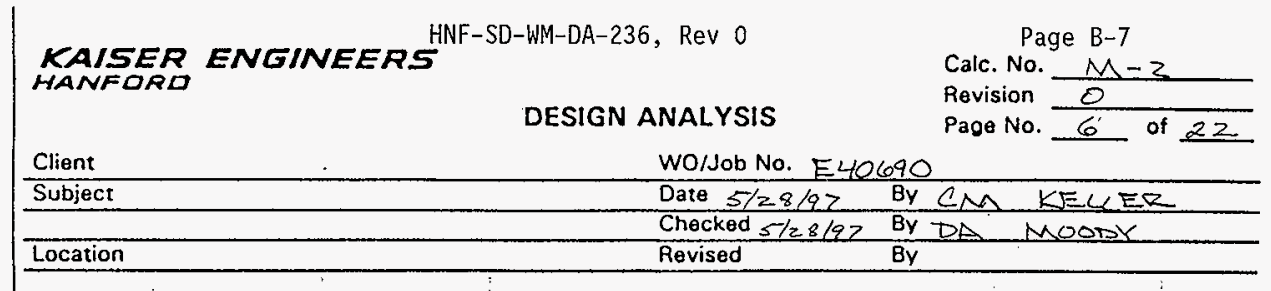

CHECK STRESS IN WELA OF TITIE TO BASE PLATE" ALOWABLE WELA STRESSES

TENSION $G_{\text {ACC }}=.6 S_{y}=06(30 \mathrm{ksi})=18 \mathrm{ksi}$

shear $7_{\text {a L }}=.4 S_{y}=.4(30 \mathrm{ksi})=12 \mathrm{ks}$.

1/4"-FULET WELT $A=.707 \mathrm{~h} \pi D=.707(1 / 4)(\pi)\left(4,5^{\prime \prime}\right)$

$A=2.50 \operatorname{in}^{2}$

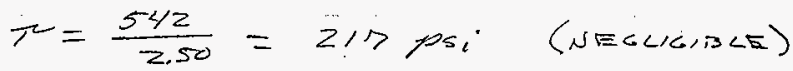

NOTMAL STRESS DUETO BENDING $\angle=\frac{M C}{1}$

$$
I=.707 \mathrm{i} I_{4} \quad \text { uft, sect } 9-4
$$

$$
I_{4}=\pi r^{3}=\pi(2.25)^{3}=35.78 \mathrm{in}^{3}
$$

$F=.707(1 / 4)(35.78)=6.32 \mathrm{in}^{3}$

$\zeta=\frac{(z 2>>3)(z .25)}{k .32}=8107 \mathrm{ps}^{\circ}<18 \mathrm{ks} \quad$ oK

$\therefore 1 / 4, \cdots$ FILLET WELA IS ACCEPTABLE

54-4300-037 KEH-0037.00 (06/92) 


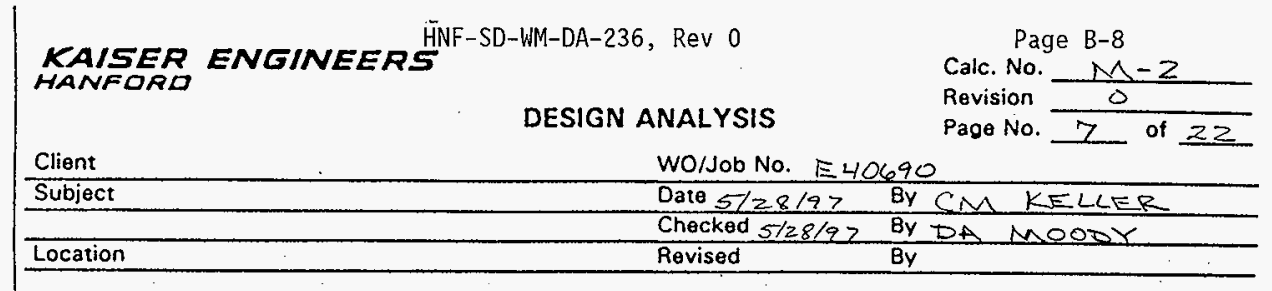

CHECK STRESS IN CONCRETE ANCHORS AND BBASE PLAFE:

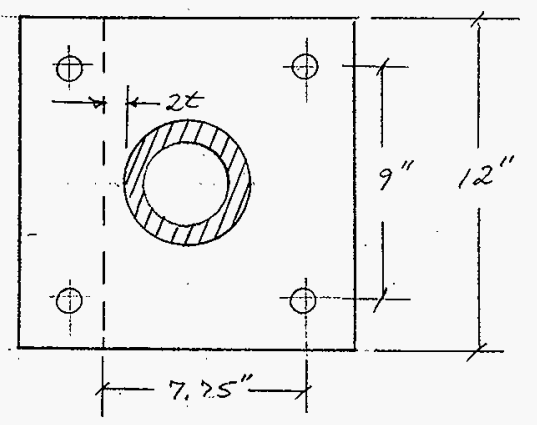

PLATE THAKNES $\tau=.5$

use conservative banding to occur a distance $2 t$ from Pinc o.D.

ANCHORS ARE $\phi 5 / 8$ HLTI KWIK-BOLTI, 23/4" EMBEOMENT, CONCRETE is 3000 psi mminum cempressive sTRENKTH MINIMUM ANCHCR SPACINC: 8.03": OK ref 9,5TD, MINIMUM EDGE DISTANCE: 8.26" OK

EMBEDWET

ALCOWABLE LOADS PERANCHCR

$$
\begin{aligned}
& T_{\text {ALC }}=1749^{7} \\
& V_{\text {AL }}=2518^{\prime 2}
\end{aligned}
$$$$
\frac{V}{V_{A L C}}+\frac{T_{1}}{T_{B L C}} \leq \% 0
$$$$
V=\frac{542}{4}=136
$$

$$
\begin{aligned}
& M=2 T\left(775^{\prime \prime}\right) \\
& 22773 \text { in } 16=2 T\left(7.75^{11}\right) \Rightarrow T=1469^{*} \\
& \frac{136}{2518}+\frac{1469}{1749}=.89<1.0 \quad 0 K
\end{aligned}
$$

ANCHORS HRE ACCEPTABLE 54.4300-037 KEH-0037.00 106/92) 


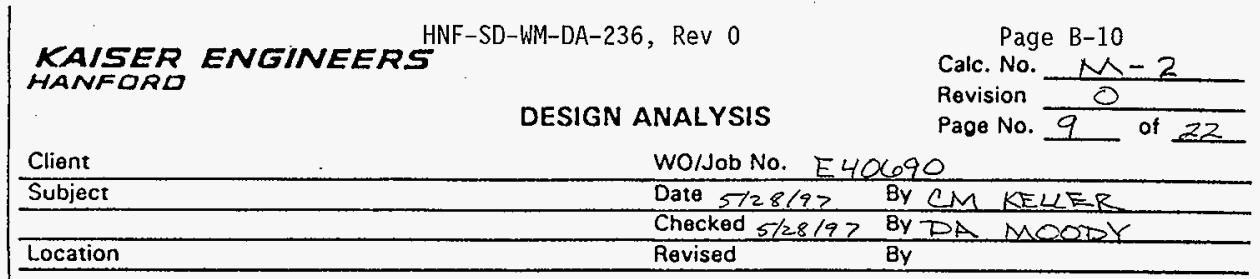

ANALYZE HORIZONTAL STRUT PIPE SUPPORT:

HORIZONTAL REACTION LOAD FROM ALTOPIPE ANALYSIS

SUPPORT B LE $\quad F_{Z}=614^{\#}$

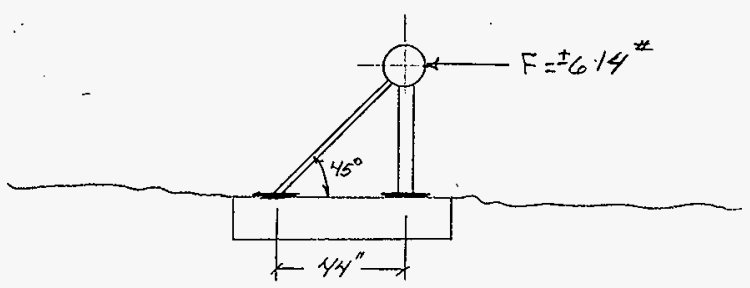

FORCE ON STRUT: $F=\frac{614}{.707}= \pm 868$

ALLOWABLE STET LOND, IS $4500^{\circ}$

$868^{*}<4500$ OK

CHECK ANCHORAGE OF SUPPORT TO CONCRETE BASE
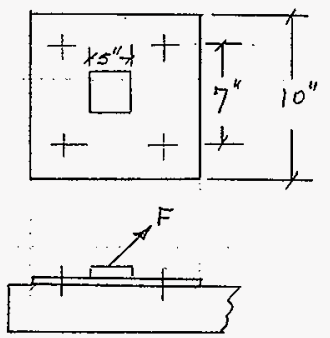

$1 / 4 "$ "THE ABC PLATE

$$
\begin{aligned}
& F=911^{*} \\
& T=.707 F=644^{k} \\
& V=.707 F=644 *
\end{aligned}
$$

BASE PLATE ANCHORED TO CONCRETE USING Ф $3 / 8$ HILT I KWIK BOLT $W / / 5 / 8 "$ EMBEDMENT.

$54-4300-037 \quad \mathrm{KEH}-0037.00(06 / 92)$ 


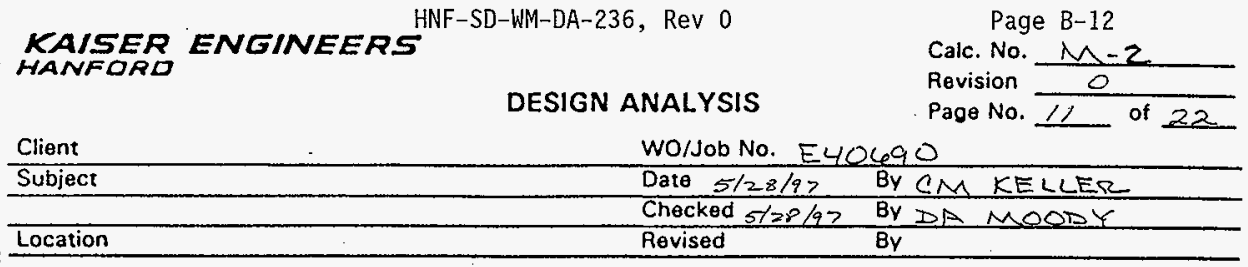

ANALYZE WIND LOADING ON DUCT WORK:

$$
\begin{aligned}
& F=q_{2} G_{h} C_{f} A_{f} \quad \text { yo } \\
& q_{z}=.00256 \quad K_{z}(I V)^{2} \\
& K_{z}=.80 \text { TABLFL } \\
& I=1,0 \\
& V=80 \mathrm{MPH} \\
& q_{z}=.00256(.80)(1.80)^{2}=13.1 \\
& G_{h}=1.32 \text { TABLE EXP, } \\
& C_{f}=1.2 \text { TAB } \\
& A=(1 \%)\left(1^{\prime \prime}\right)=.83 \mathrm{fz}^{2} \text { pen ff of insulated der } \\
& F=(13.1)(1.32)(1.2)(.83)=17.22 * / f t \text { of ere }
\end{aligned}
$$

For 2O' SPACINC BETWEEN SUPPORTS THE HORIZONTHL FORLE DUE TO WIND LOADING IS $\left(20^{\prime}\right)\left(17,22 z^{*} / t_{t}\right)=344^{*}$

DETERMINE STRESS ON $\phi 6^{\prime \prime}$ DUCT PIPING DUE TO WIND LOADING:

ANALYZE PIPE AS SIMPLY SUPPORTED BEAM SUBJECTED TO DISTRIBUTED LOADING $W=17.22 \mathrm{k} / \mathrm{fr}$

54-4300-037 KEH-0037.00 (06/92) 


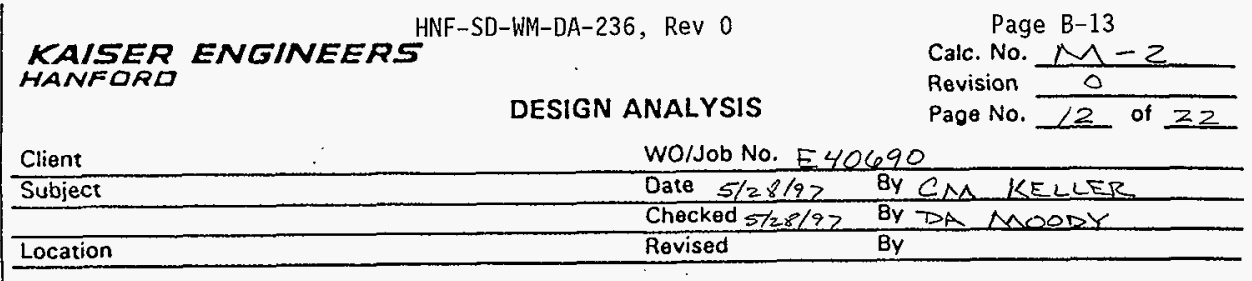

$$
\begin{aligned}
& 6 " \text { sch } 40 \text { pipe: } I=28 \text { in } \\
& 0 . D=C .625 \Rightarrow C=3.313 \\
& M=\frac{\omega l^{2}}{8}=\frac{17.22(20)^{2}}{8}=861 \text { fz.16 wm } \\
& s=\frac{M c}{I}=\frac{(861 \mathrm{ft} .13)(12 \mathrm{~m} / \mathrm{ft})(3.3131)}{28 \mathrm{in}^{\prime \prime}}=1223 \mathrm{psi} \\
& 1223<21 \times 5 i \quad 0 k
\end{aligned}
$$

$\therefore$ Pipe wILL NOT BUCKLE DUE TO WIND LOADING

$54-4300.037 \quad \mathrm{KEH}-0037.00(06 / 92)$ 
KAISER ENGINEER HNF-SD-WM-DA-236, Rev 0 HANFORD

Client

Subject
DESIGN ANALYSIS
Page B-14

Calc. No. $M-z$

Revision 0

Page No. 13 of $z 2$

Wo/Job No. E40690

Date $5 / 28 / 97$ Checked $5 / 28 / 98$

BY CM KELLER

Revised BY

EVALLIATE STRESS ON EXHAUSTER INLET

USE RESTRAINT REACTIONS FROM ALTOPIPE ARIALSIS POINT ...A.OO.N ATTACHMENT.

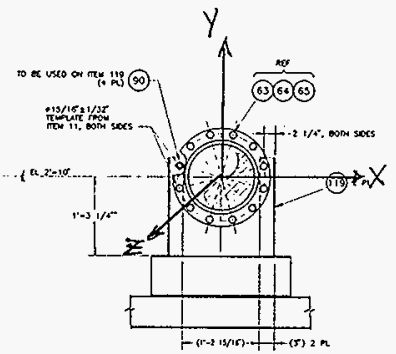

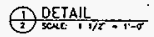

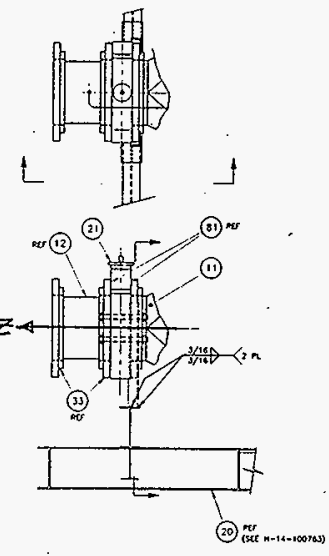

CHECK STRESS ON INLET SUPPORT: (PN 119 ABOVE). Support is $3 \times 2 \times 1 / 4$ CS ANGLE ASTM A 36 FNLLE FOPERTFS: NH 3

$$
\begin{aligned}
& A=1119 \sin ^{2} \\
& \sigma_{y}=36 k s_{\circ} \\
& I_{x x}=.392 \mathrm{is}^{4} \\
& s_{x x}=.260 \mathrm{in}^{3} \\
& I_{Z_{2}}=1.09 \text { in } 4 \\
& S_{z z}=.542 \text { in }^{3} \\
& \sigma_{p e c}=.6\left(\alpha_{y}\right)=22 k s
\end{aligned}
$$




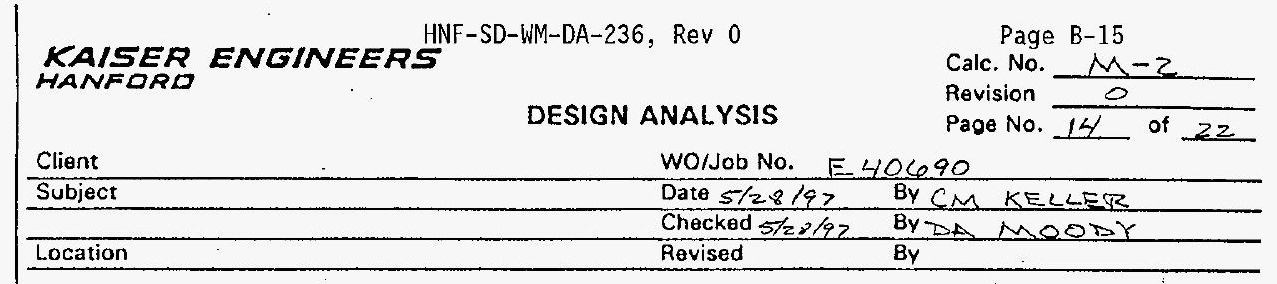

REACTIONS ON EXIPHUSTER INCET: (FROM AUTOPIPE AWALYSIS)

$$
\begin{array}{ll}
F_{x}=30 & M_{x}=10.5 \mathrm{ft} \cdot 1 \mathrm{~b} \\
F_{y}=302^{*} & M_{y}=16 \mathrm{ft} \cdot 1 \mathrm{~b} . \\
F_{z}=45 & M_{z}=115 \mathrm{ft} \cdot \mathrm{lb}
\end{array}
$$

Reackions on inlet support are as follows:

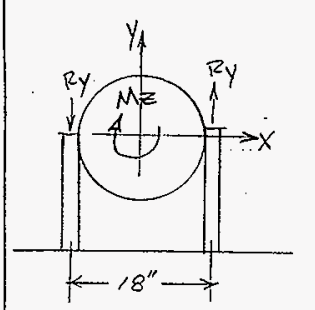

Reaction tue to $M_{z}$ :

$$
1 / 5 \cdot f \cdot 16=2 R_{y}\left(9 / 1 z^{\prime}\right) \Rightarrow R_{y}=77^{*}
$$

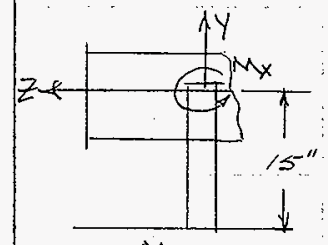

Rectorion Due to $M x$

$$
105 \mathrm{Af}_{1 / 6}=2 R_{z}\left(15 / 1^{\prime}\right) \Rightarrow R_{z}=42^{*}
$$

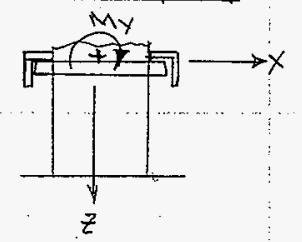

Reaction tove to My

$$
16 \text { ft:l6 }=2 R_{z}\left(\frac{18}{12} \Rightarrow R_{z}=5^{*}\right.
$$

TOTAL SUPFORT FORCES:

$$
\begin{aligned}
& F X=30^{*}+ \\
& F Y=302^{*}+77=379^{4} \\
& F Z=45^{*}+42+5=92
\end{aligned}
$$

$54-4300-037$ KEH-0037.00 (06/92) 


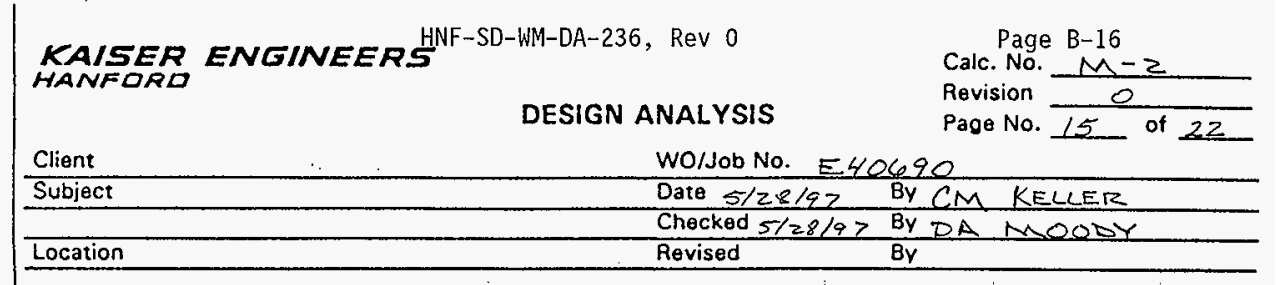

For Combined compression and bending stresses,

$$
\frac{\sigma_{a}}{s_{a}}+\frac{s_{b x}}{s_{b x}}+\frac{\sigma_{b z}}{s_{b z}} \leq 1 \quad \text { w } \frac{3}{f}+1-3
$$

when $\sigma_{a}=$ Axial Compressive Stress

$$
\begin{aligned}
& \sigma_{b_{x}}=\text { Bending stress in } x \text {-dir. } \\
& \sigma_{b z}=\text { Bering stress in } z \text {-din. } \\
& \mathrm{Sa}_{a} \mathrm{~S}_{b_{x}}, S_{b_{z}}=A \text { LLOWABLE STReSSES }=22 \mathrm{ks} \mathrm{S}^{\circ} \\
& \sigma_{a}=\frac{F Y}{A}=\frac{379^{H}}{1.19 \mathrm{~m}^{2}}=318 \mathrm{ps}: \\
& \sigma_{b_{x}}=\frac{F x\left(1 s^{\prime \prime}\right)}{\sigma_{x x}}=\frac{30(15)}{260 \mathrm{in}^{3}}=1731 \mathrm{psi} \\
& \sigma_{b_{z}}=\frac{F_{z}\left(15^{\prime \prime}\right)}{s_{z z}}=\frac{92^{*}\left(15^{\prime \prime}\right)}{.54 \mathrm{in}^{3}}=2546 \mathrm{psi} \\
& \frac{318}{22000}+\frac{1731}{22000}+\frac{2546}{22000}=.21<1.0
\end{aligned}
$$

$54-4300-037$ KEH-0037.00 (06/92) 


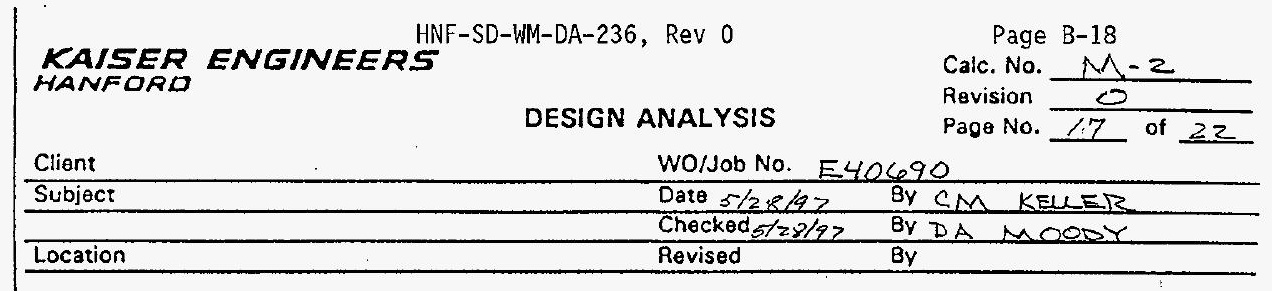

CALCULATE EXPECTED PRESSURE FROM ATMOSPHERE TO EXHAUSTER INLET FOR BOTH RUNS OF PUETWORK.

Use Saturated Air at $140^{\circ} \mathrm{F}$

USE $Q=250 \mathrm{CFM}$ PER DUCT RUN

ASSCUME " WG PreSSURE PROP FOR INLET FILTER.

DETERMINE $\triangle P$ FOR THE FOLLOWING:

1) INLET HEPA FILTER AND RISER

2) OUTLET RISER AND TEE CONNECTION

3) DUCT WORK RISER TO EXHAUSTER-LONG RUN

4) DUCT WORK RISER TO EXHHUSTER-SHORT RUN

5) EXHAUSTER INLET FITTING.

INES FILTER AND RISER (TYPICAL ARRANGEMENT)

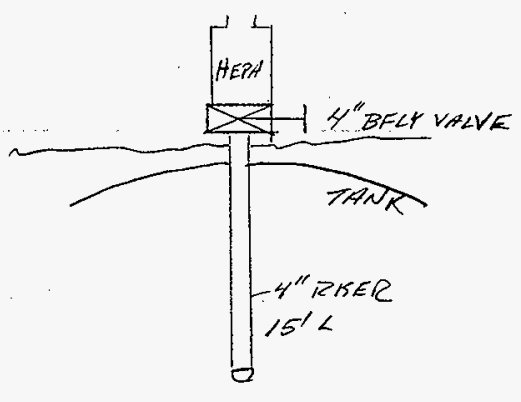

$$
\text { cosses }
$$

HERA FILTER $\triangle P=I " W L T$.

BELT VALVE $L_{e}=23^{\prime}$

TANK RISER $L=15^{\prime}$

INLET COSS Le $=20^{\prime}$

$54-4300-037$ KEH-0037.00 (06/92) 
KAISER ENGINEERS
HANFORD

Page B-23

Canc. No. $M-2$

Revision

DESIGN ANALYSIS

Page No. $\overline{22}$ of 22

Client WO/Job No. E40690

Subject Date $5 / 28 / 97$ By CM KELLER Checked s/zolar By DA Moore

Location

Revised

By

Compete $\Delta p$ for $100^{\prime}$ of 12 " pud writ $250 \mathrm{CFM}$

$$
\Delta p=.024\left(\frac{1200}{12}\right)(.0613)\left(\frac{318}{1097}\right)^{2}=.01 \text { in wh/100' }
$$

Now CALCULATE AP from ATMOSPHERE TO EXHAHSTER INLET FOR EACH RUN.

SX-1CZ LONG RUN, TANK INLET TO EXHAUSTER.

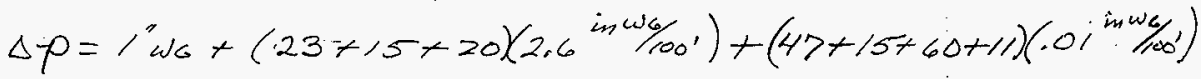

$$
\begin{aligned}
& +\left(23^{\prime}+8^{\prime}+10^{\prime}+10^{\prime}+8^{\prime}+140^{\prime}\right)\left(.35^{\text {in }} \omega_{\mathrm{kg}}^{\prime}\right)+.06 \text { in we }
\end{aligned}
$$

$\Delta p=3.28$ in war.

SX-1O3 SHORT RUN, TANK INLET TO EX HOISTER

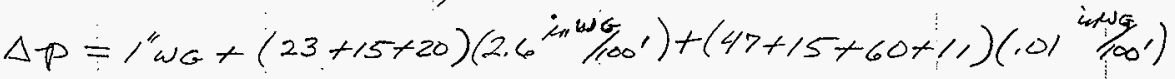

$$
\begin{aligned}
& +(23+8+10+10)\left(135 \text { in } w_{100}\right)+.02 \text { in } \omega_{6} \\
& \Delta p=2.72 \text { in } \omega / 4
\end{aligned}
$$

$\therefore$ APPROXIMATELY I/2"WG. DIFFERENCE BETWEEN LONG AND SHORT DUCT RUNS. BALANCING SHOULD NOT BE A PROBLEM. LOSS FOR TANK INLET (ATM, TO TANK DOME): Comprises THE MAJORITH OF LOSS AT 2.5 "WaG.

54-4300-037 KEH-0037.00 $106 / 92)$ 


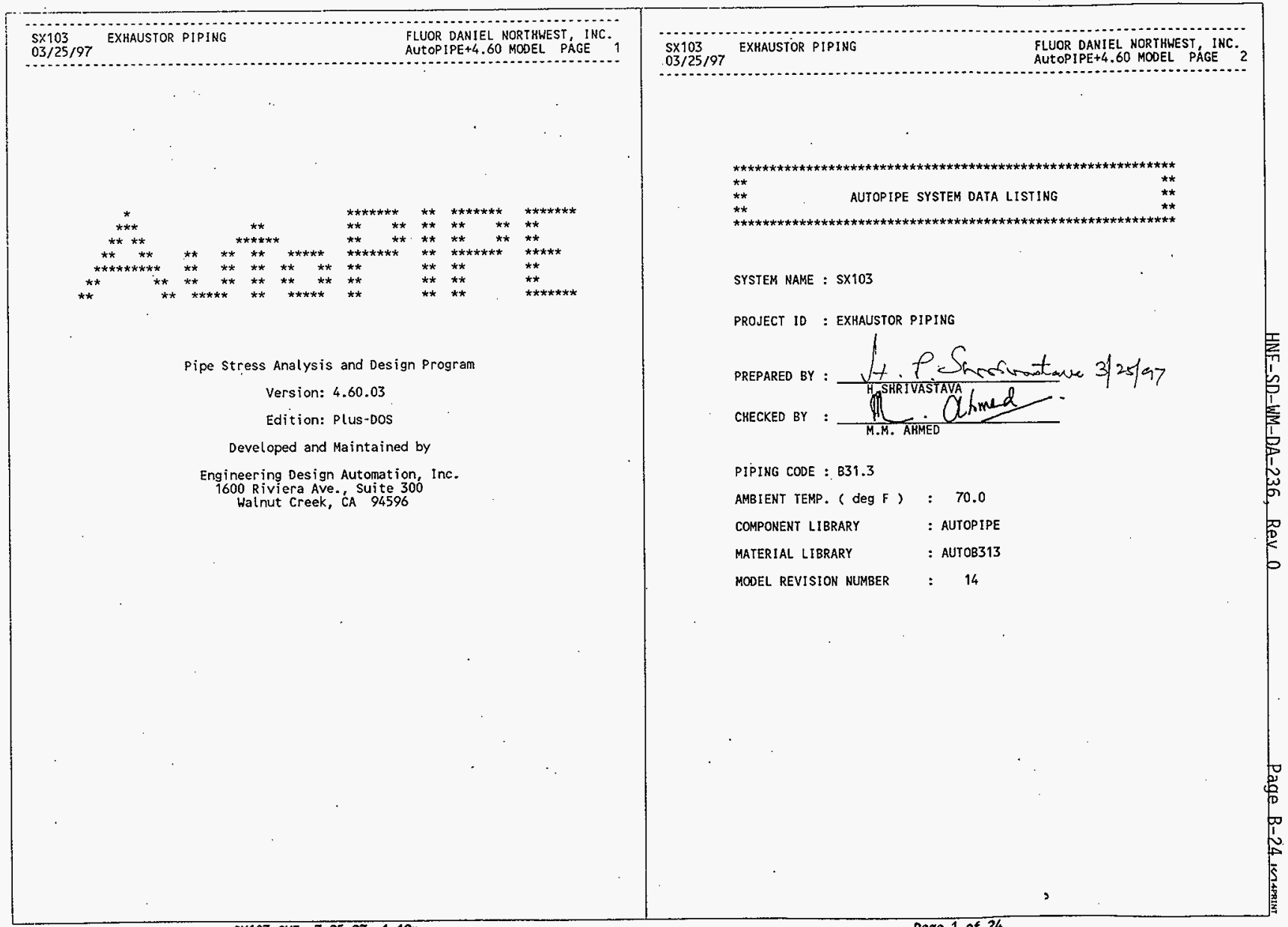




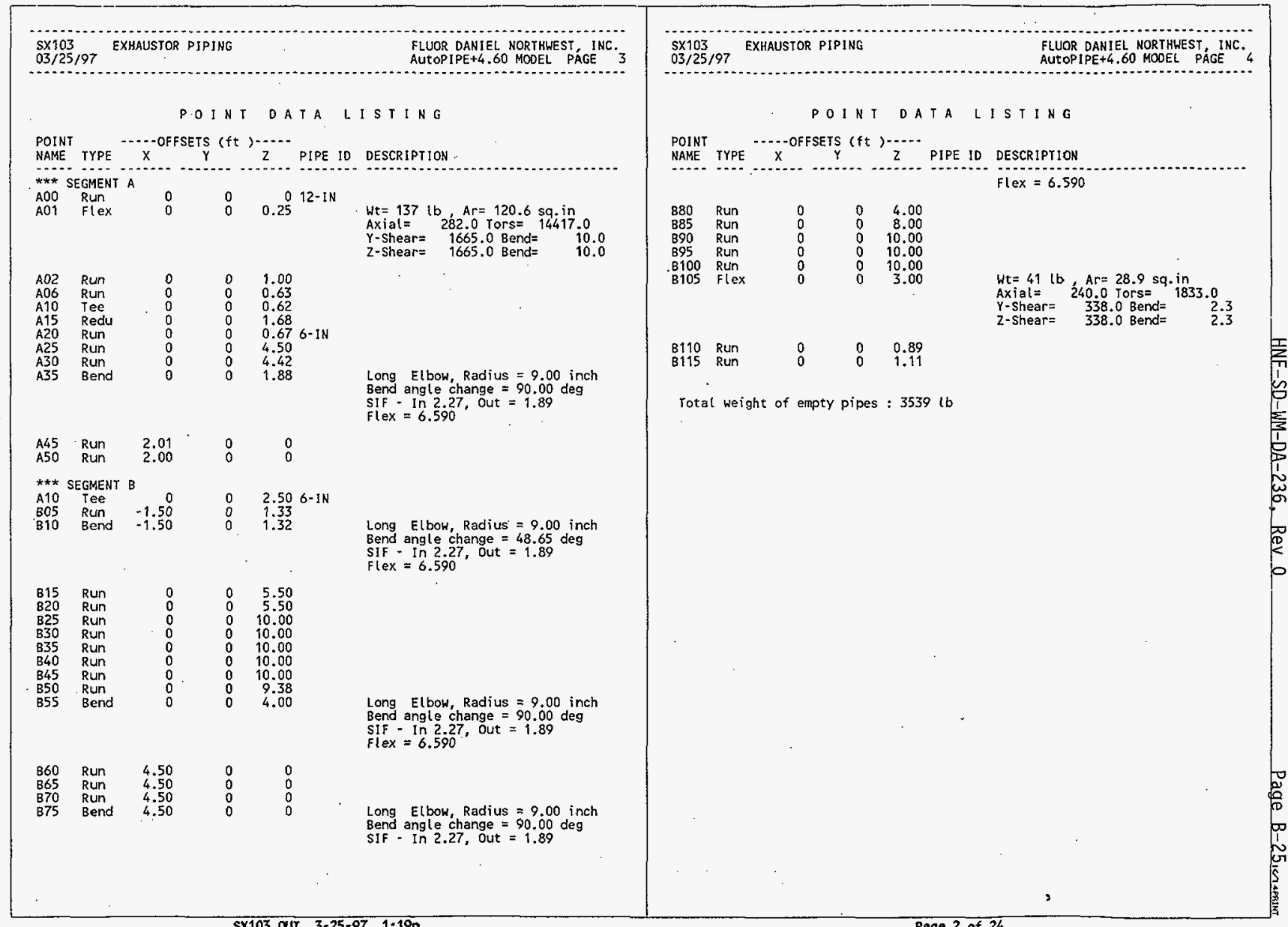




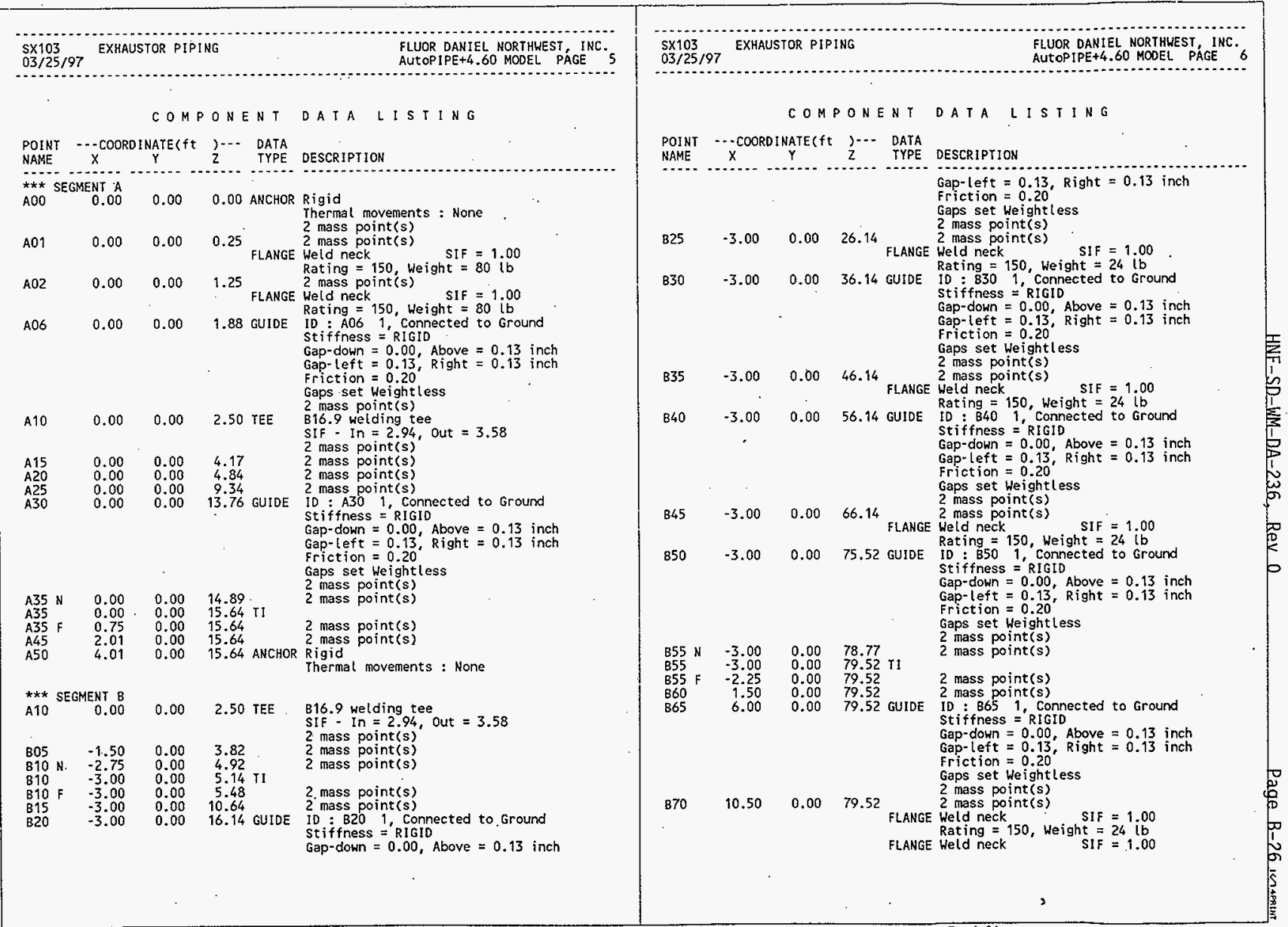




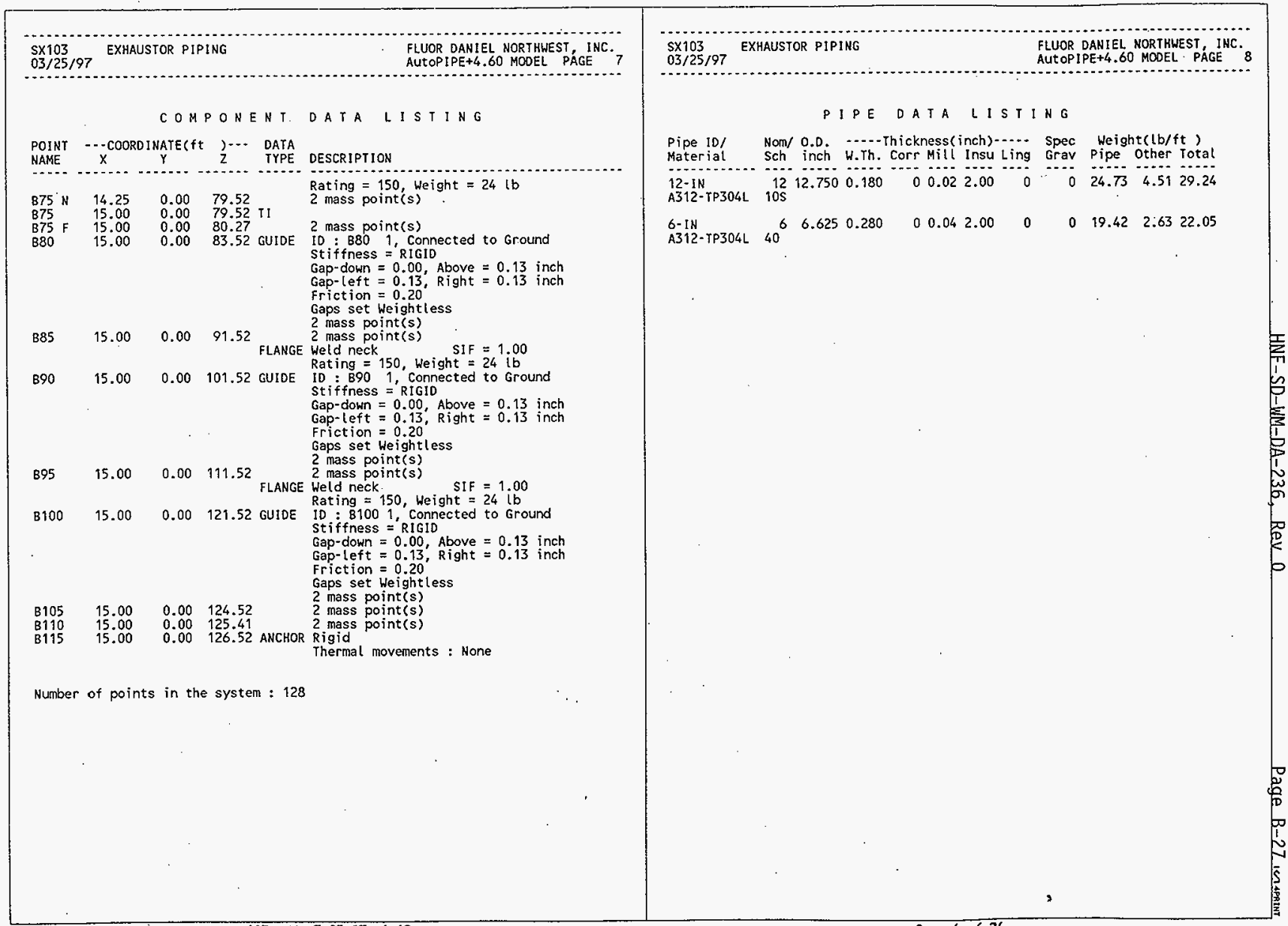




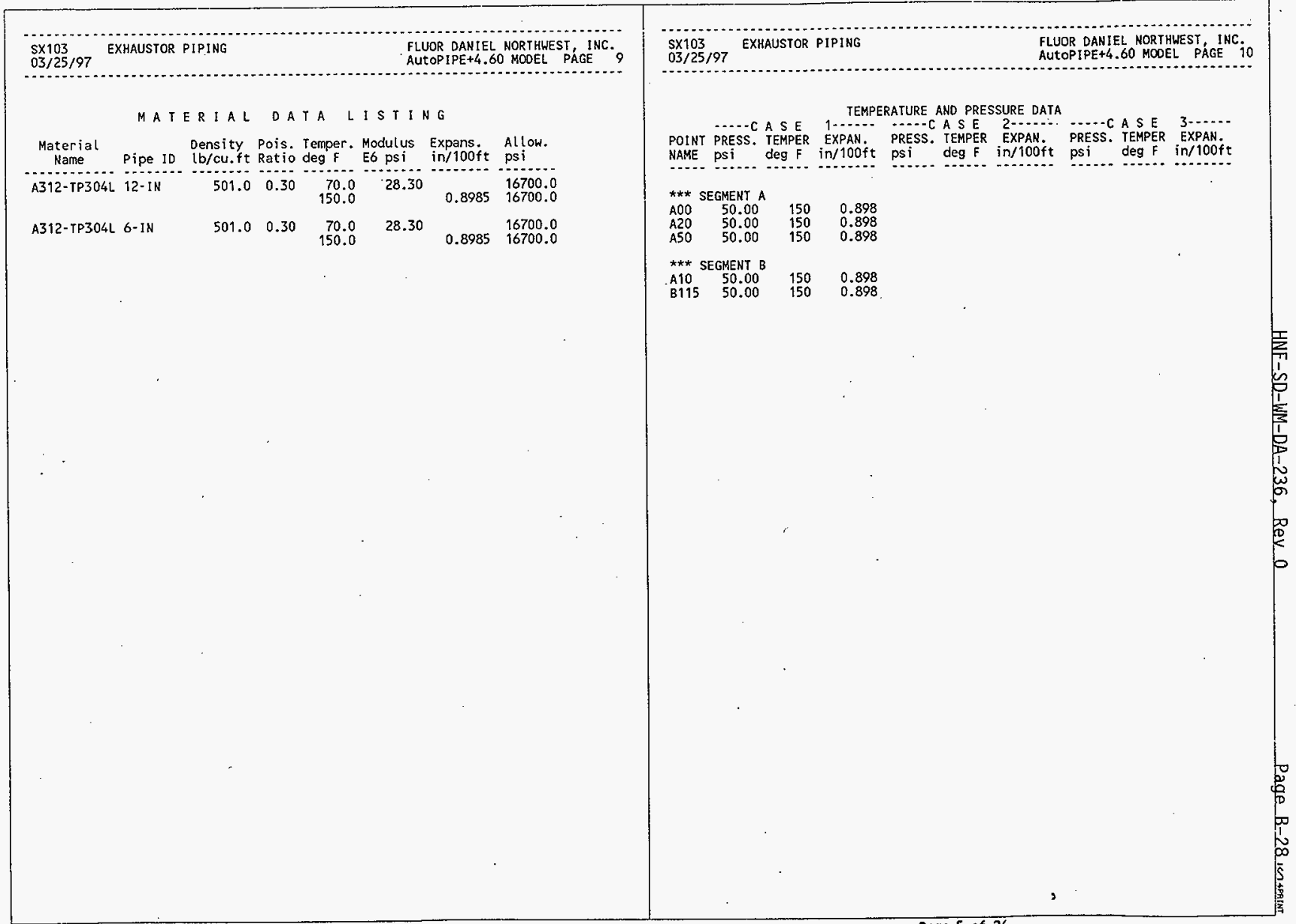




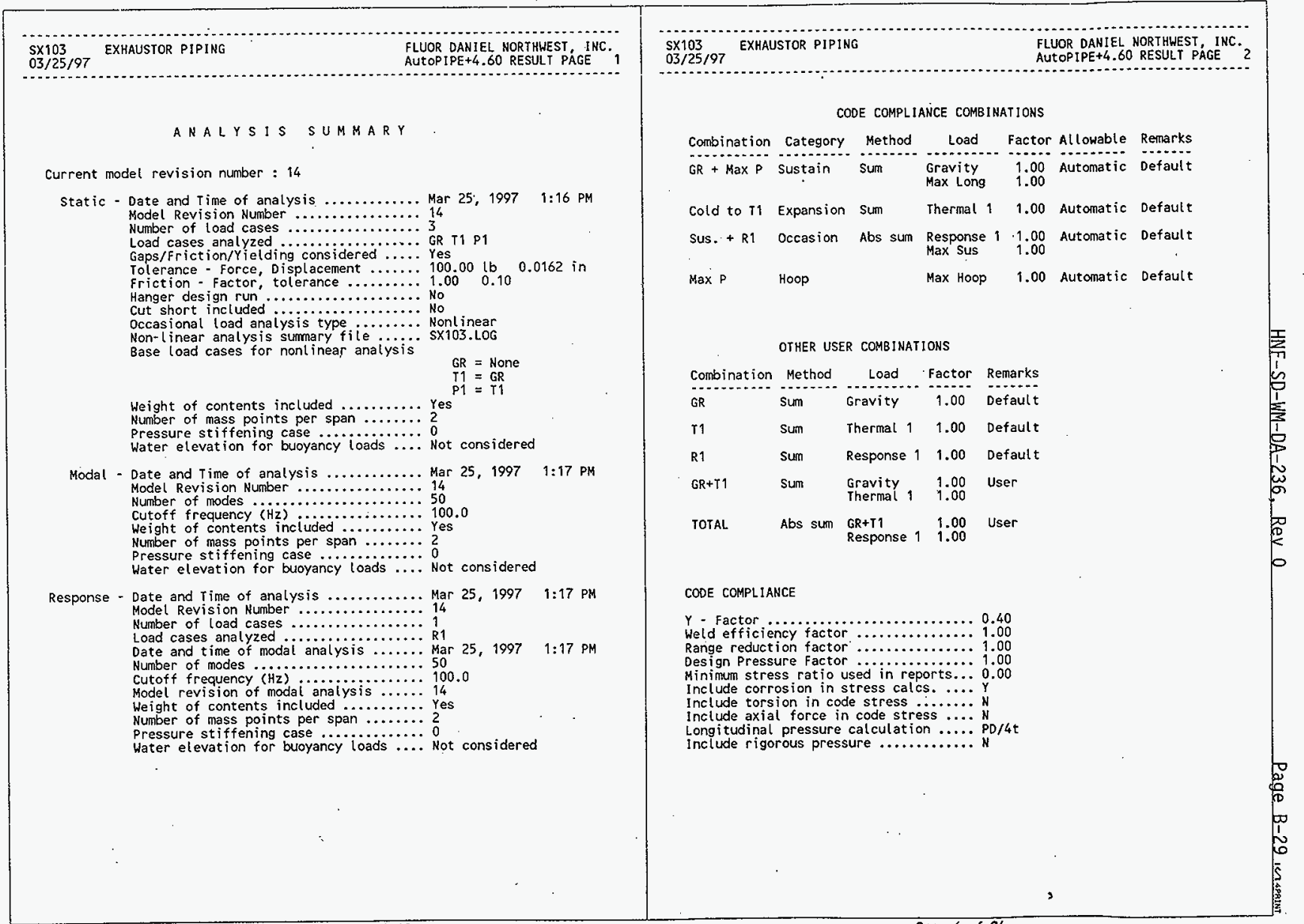




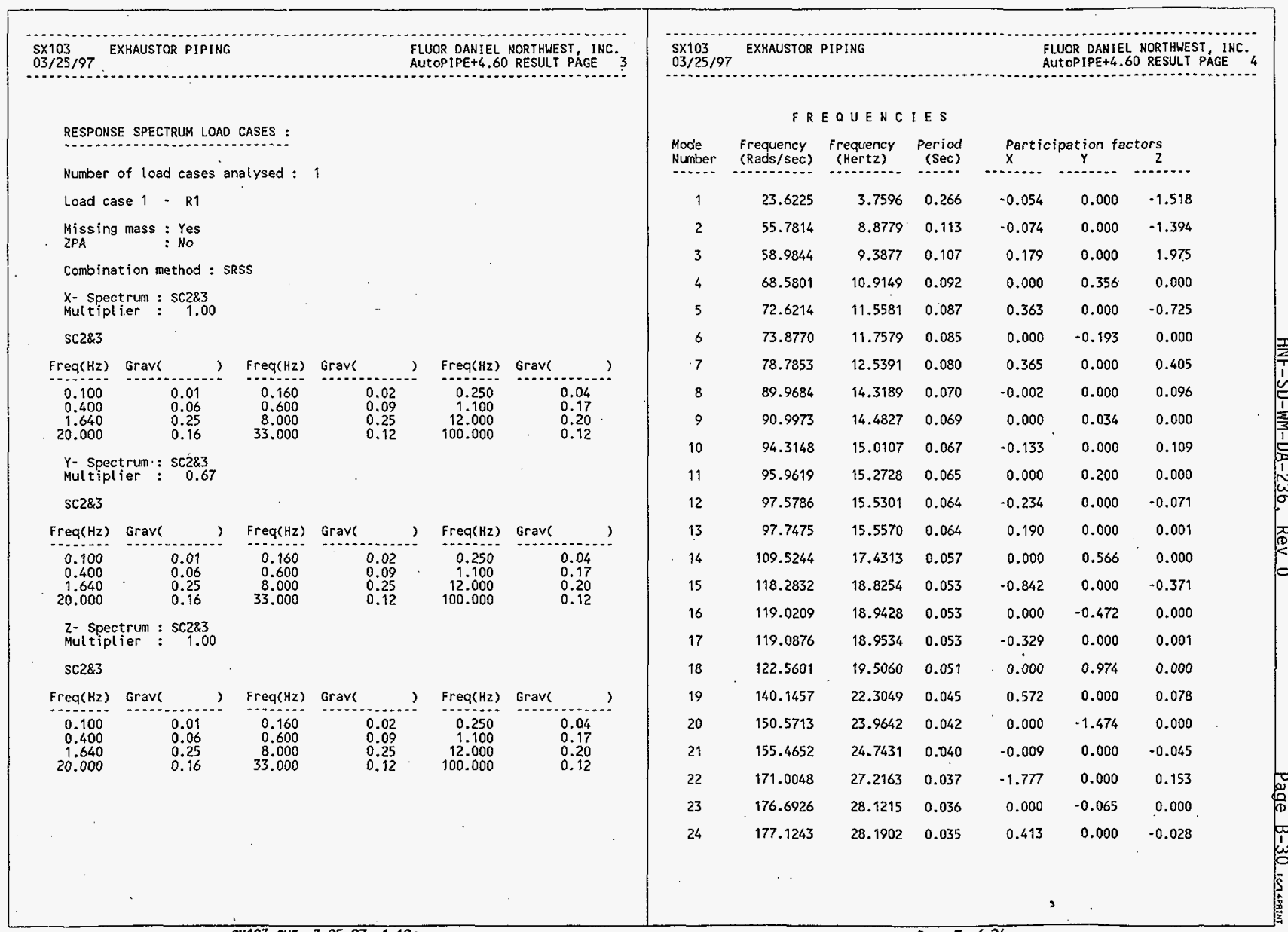




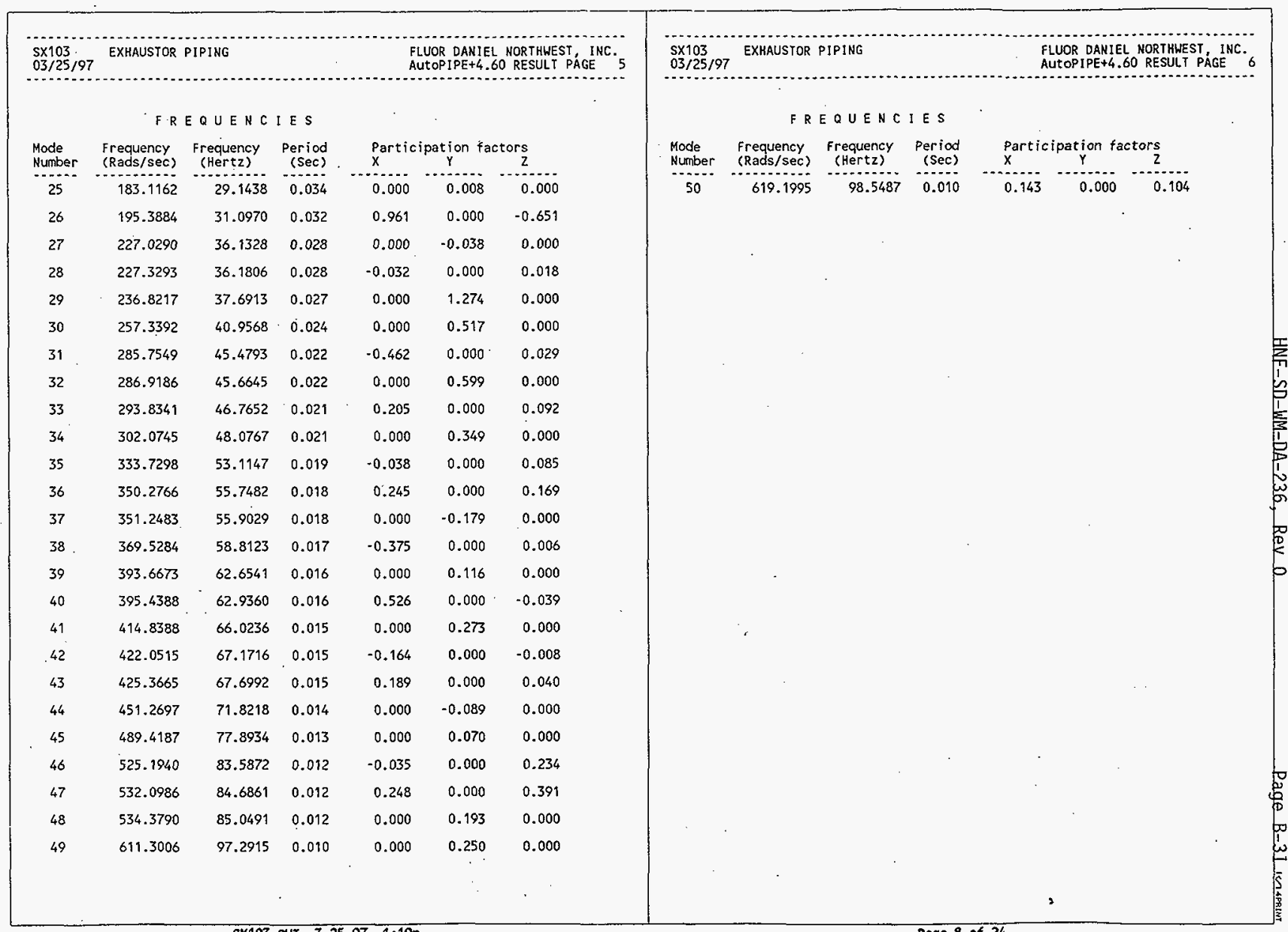




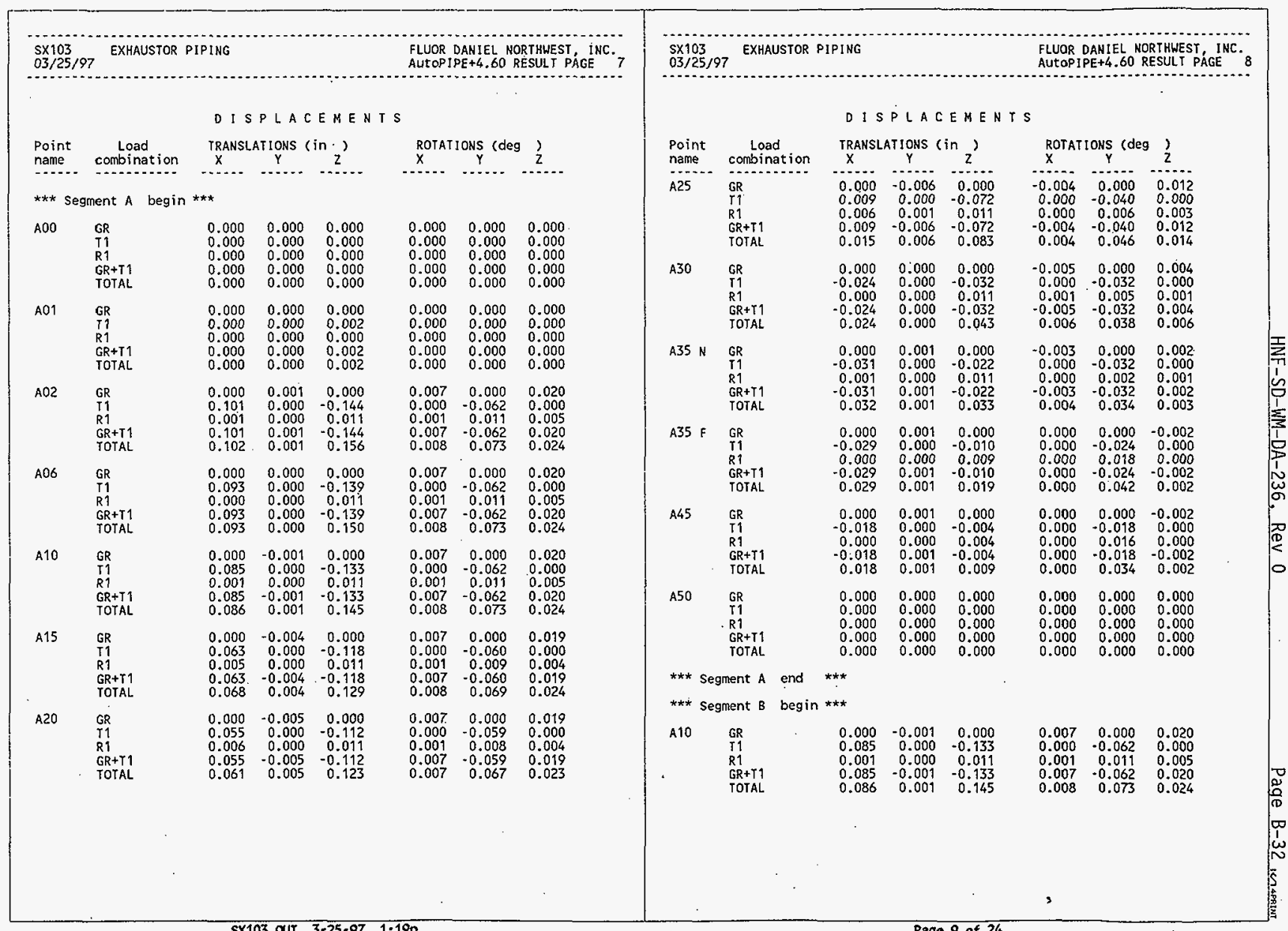




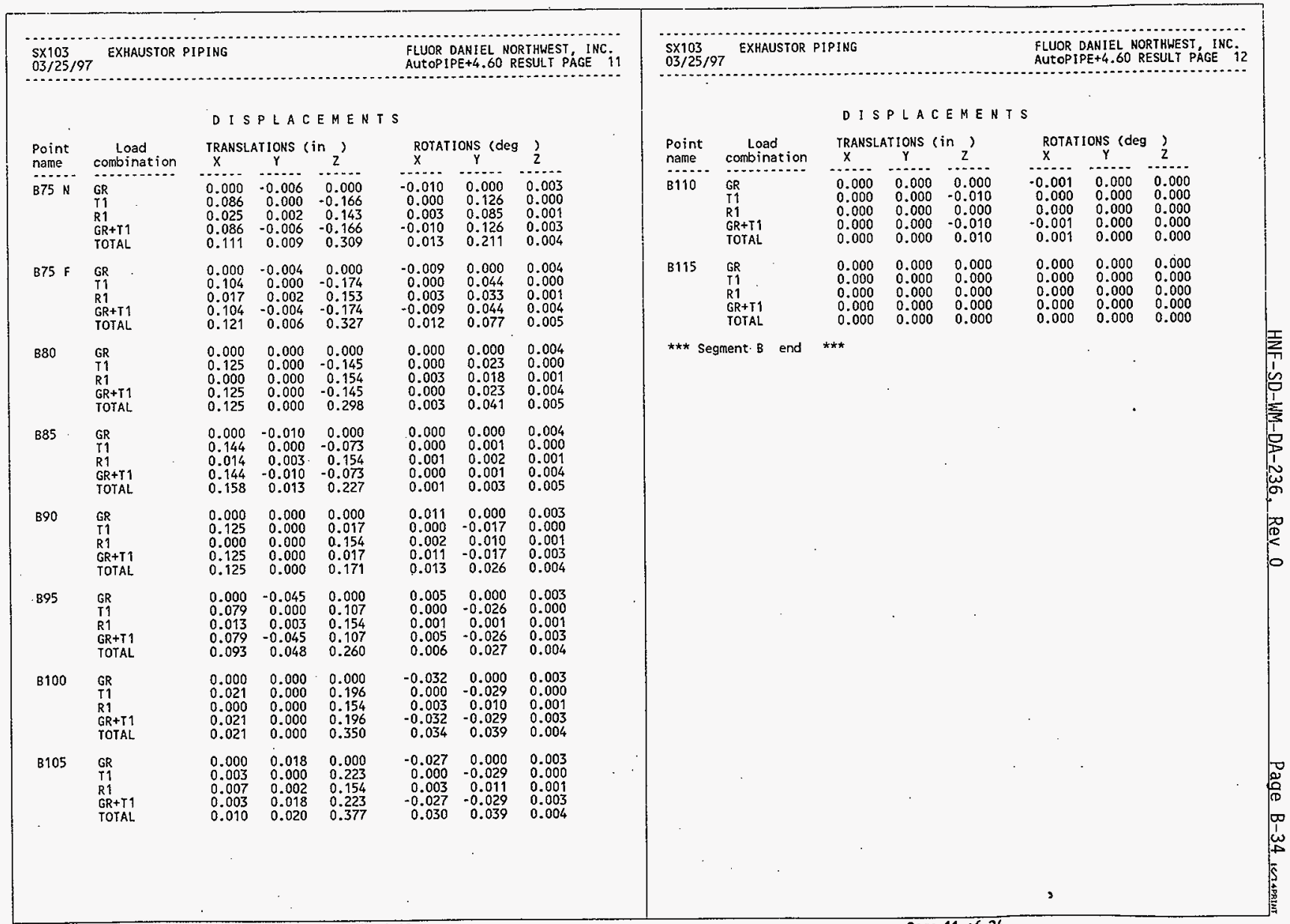




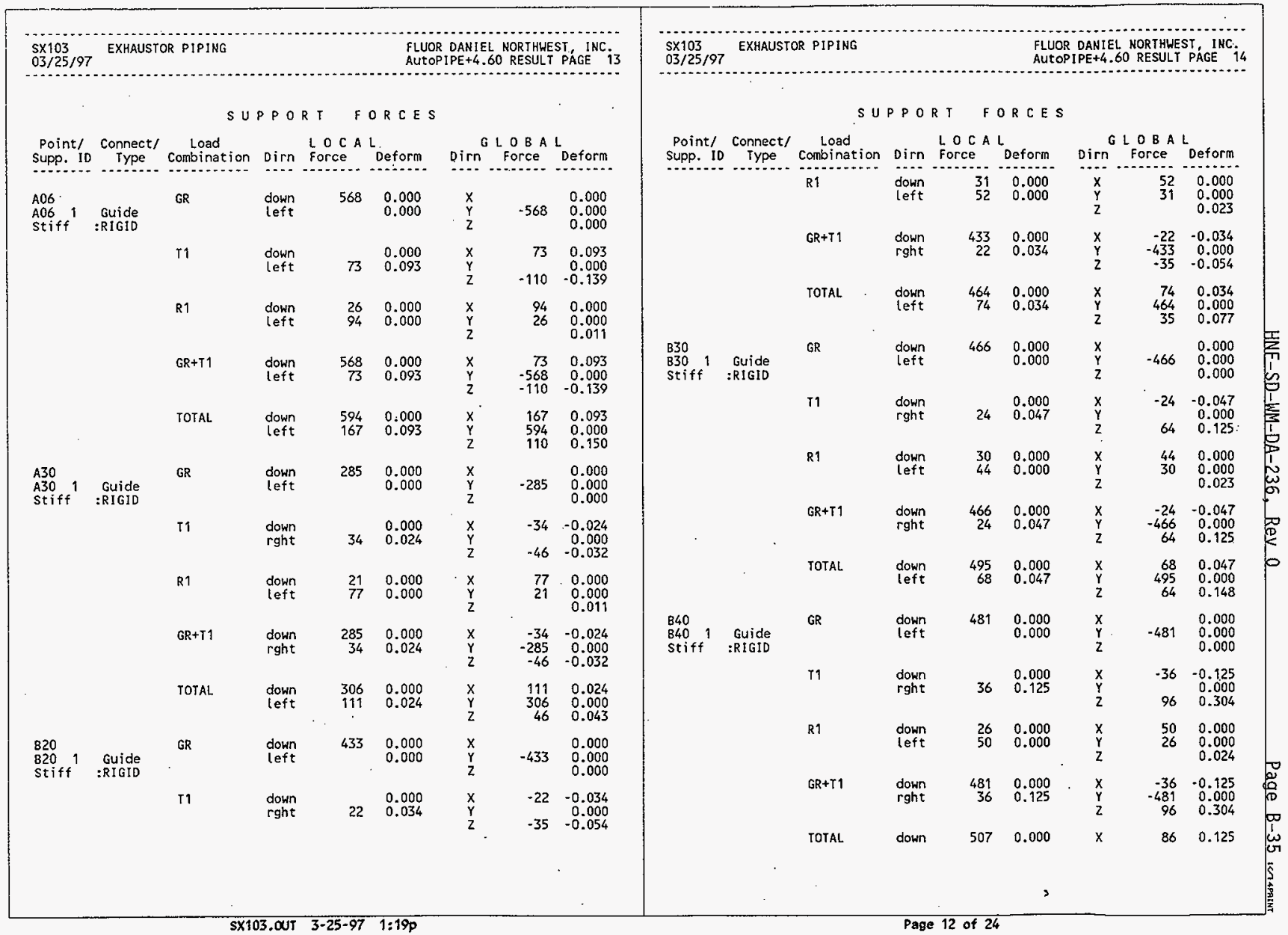




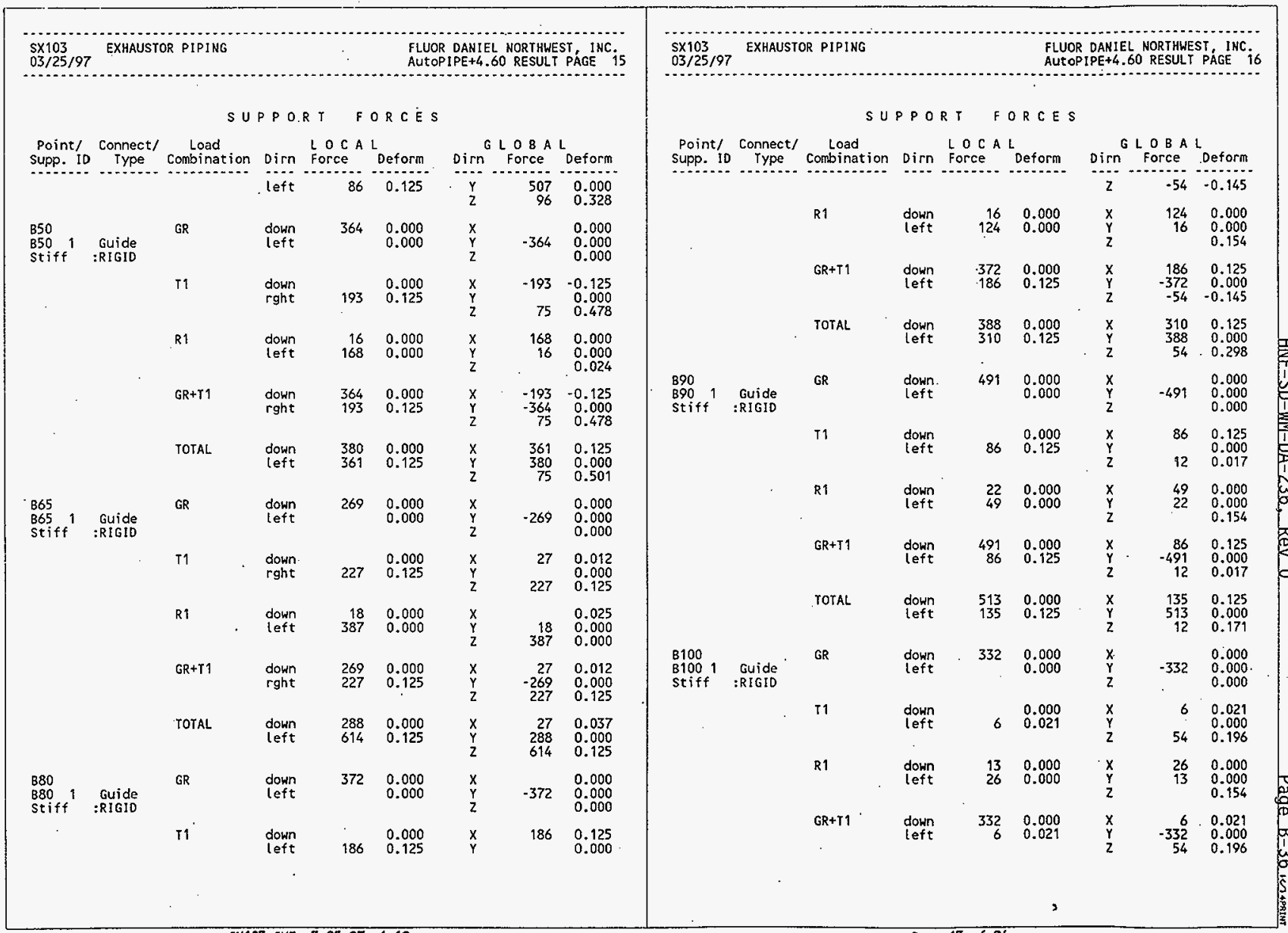




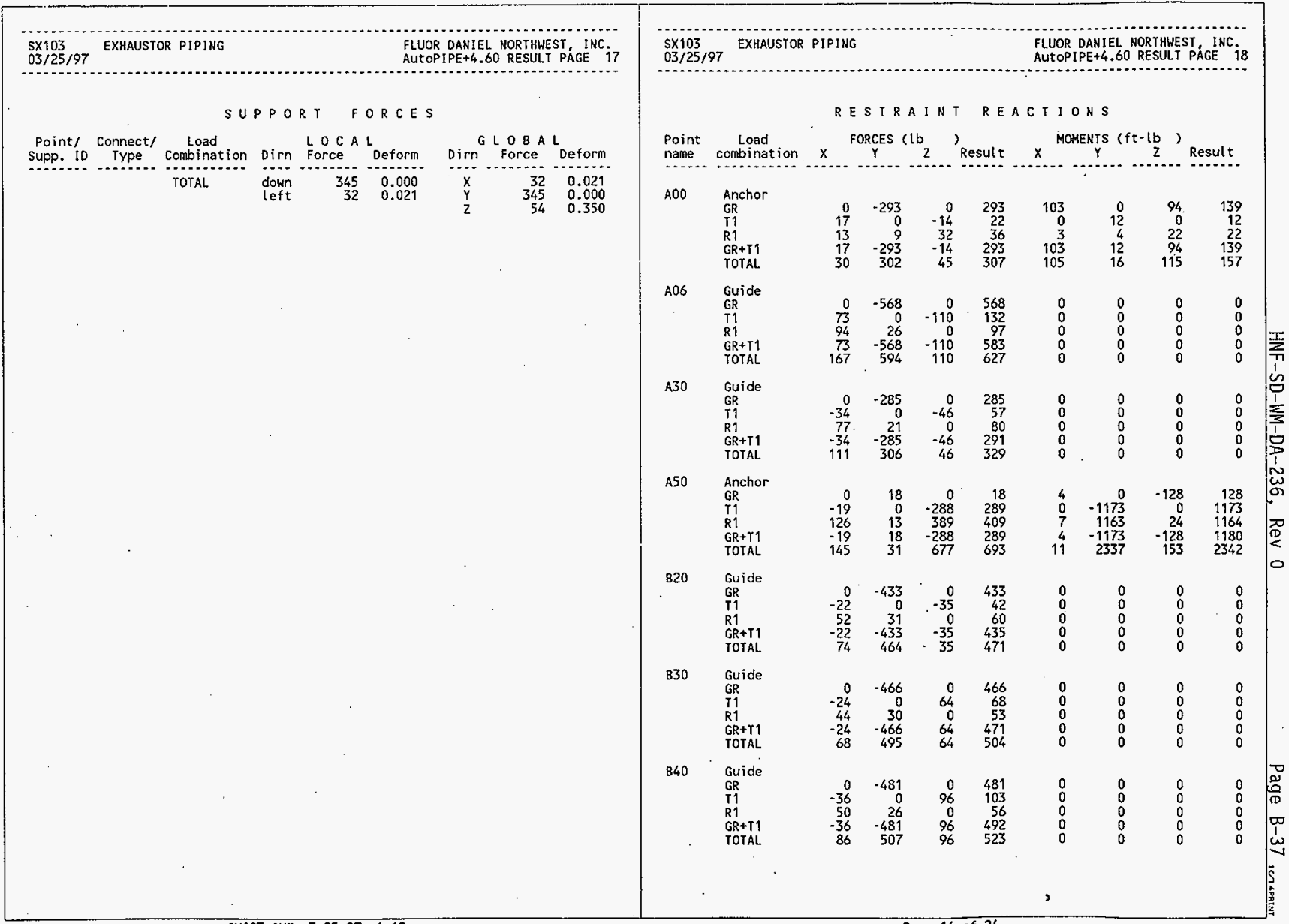




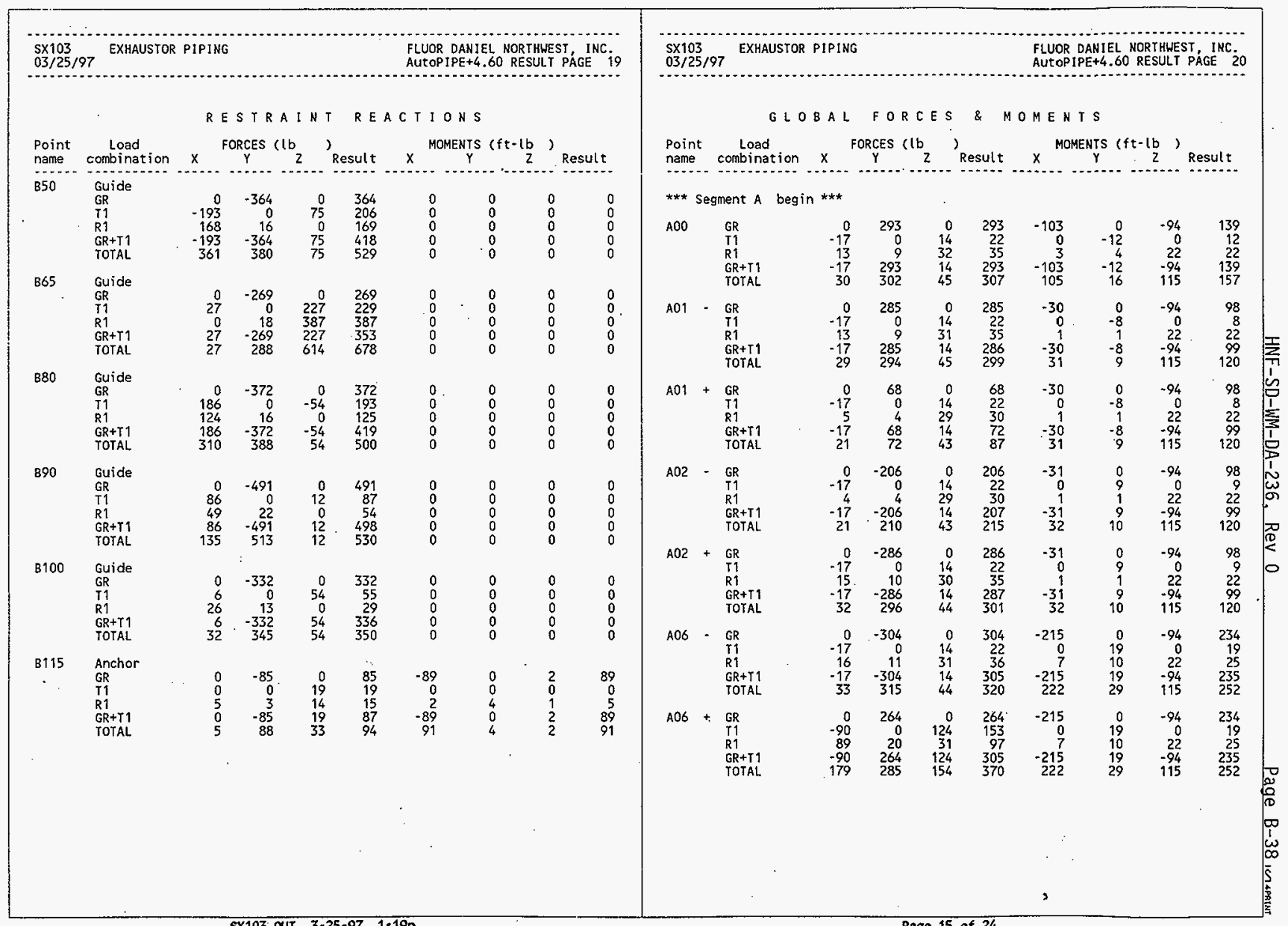




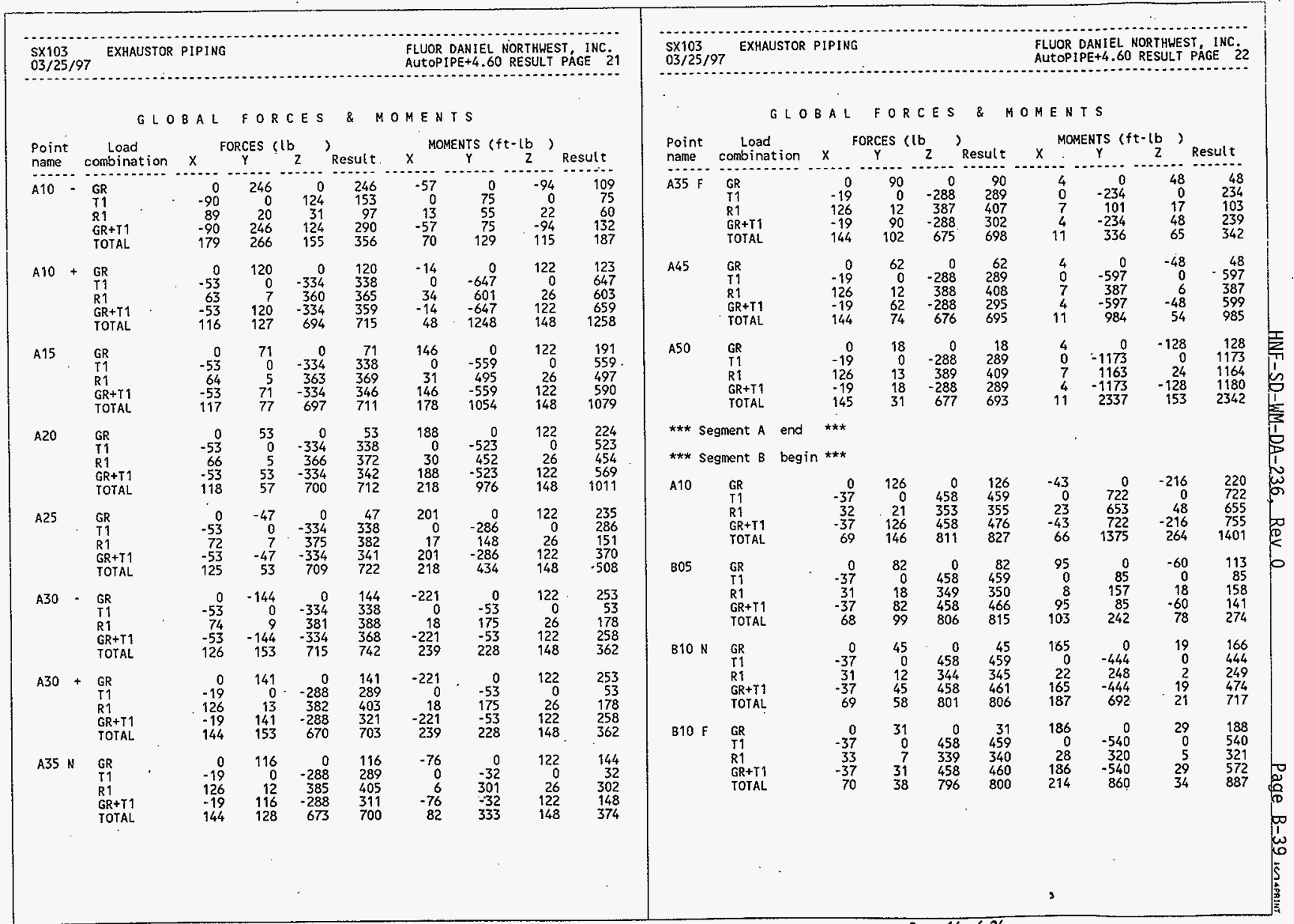




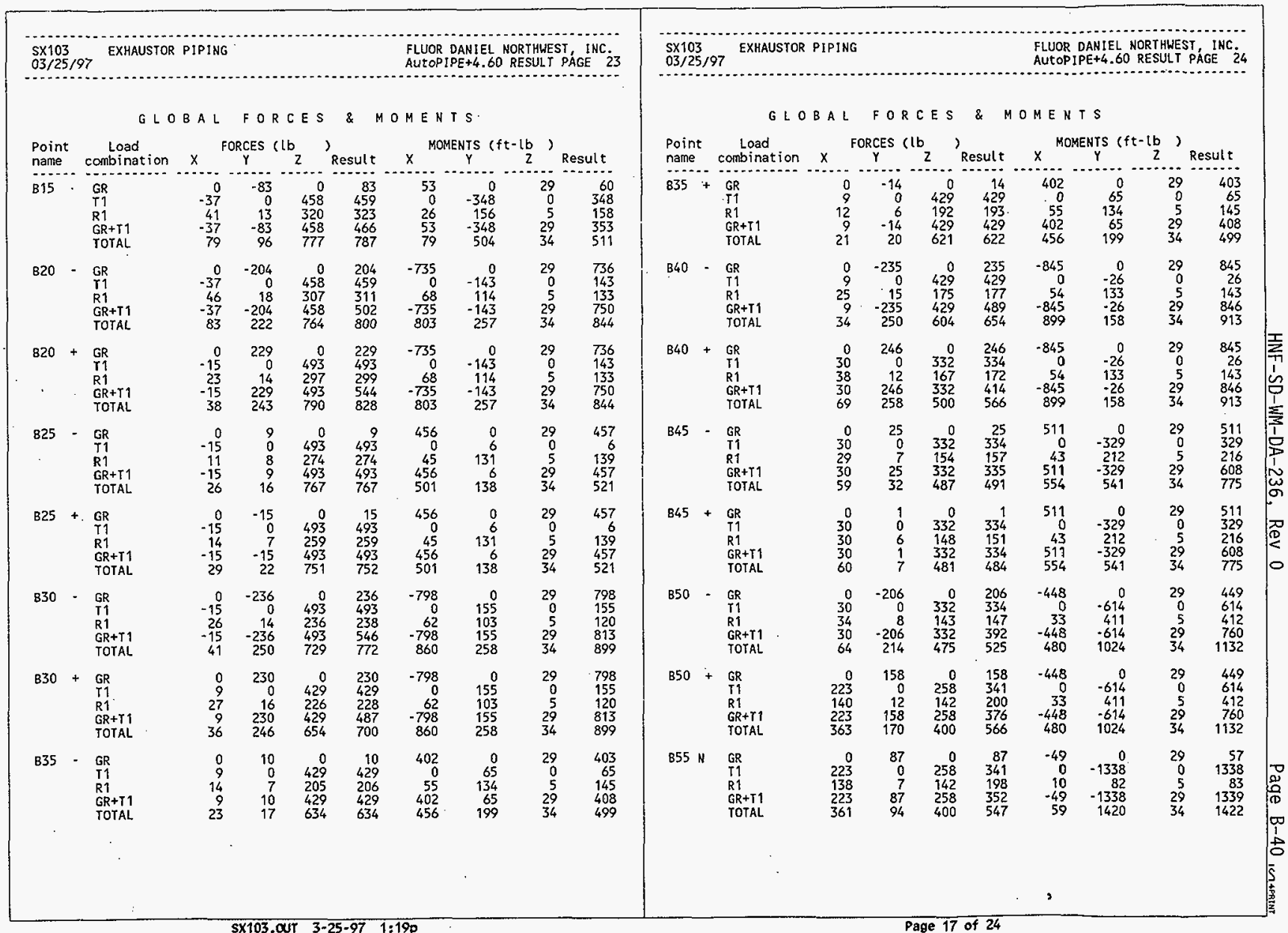




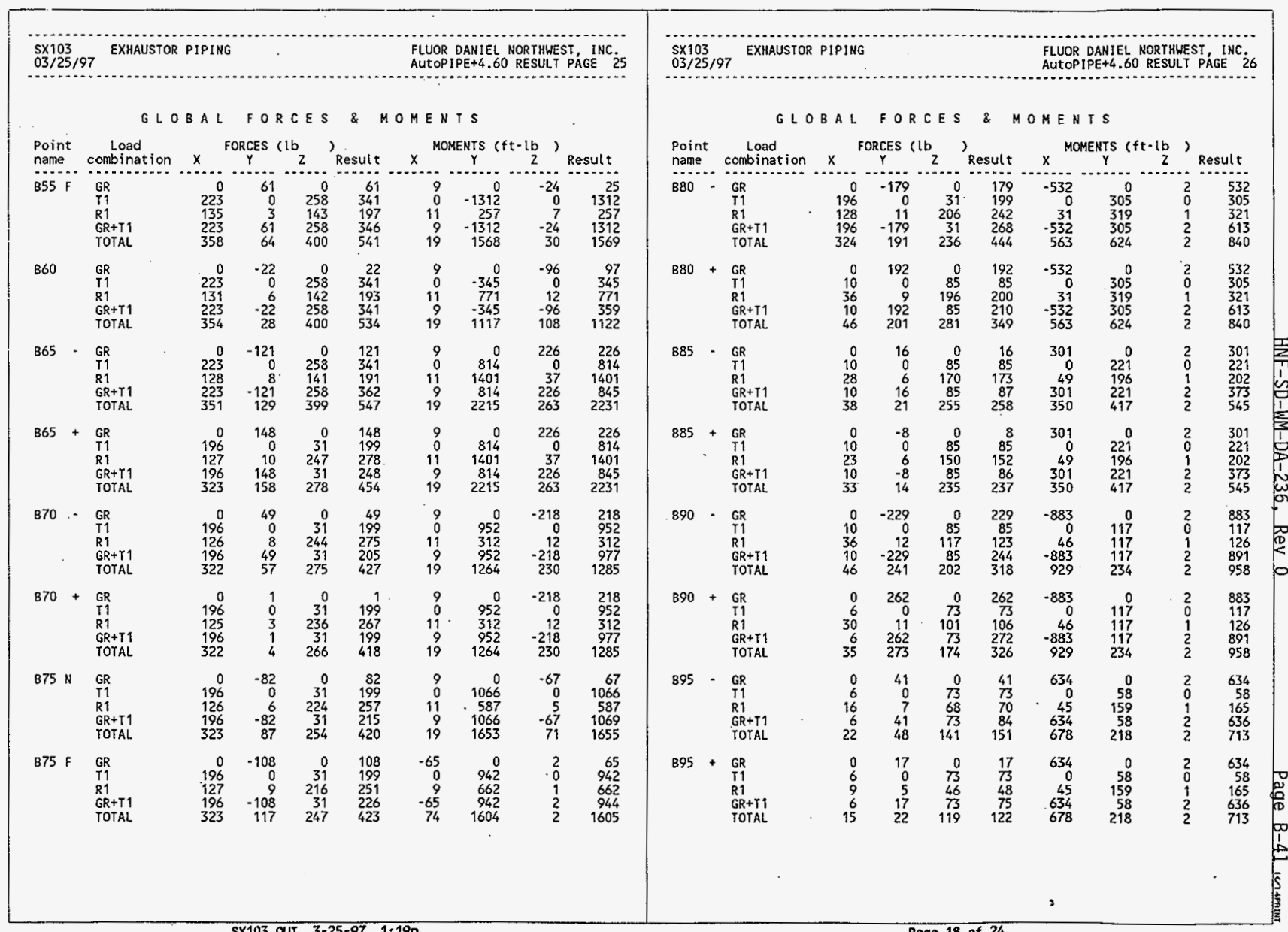




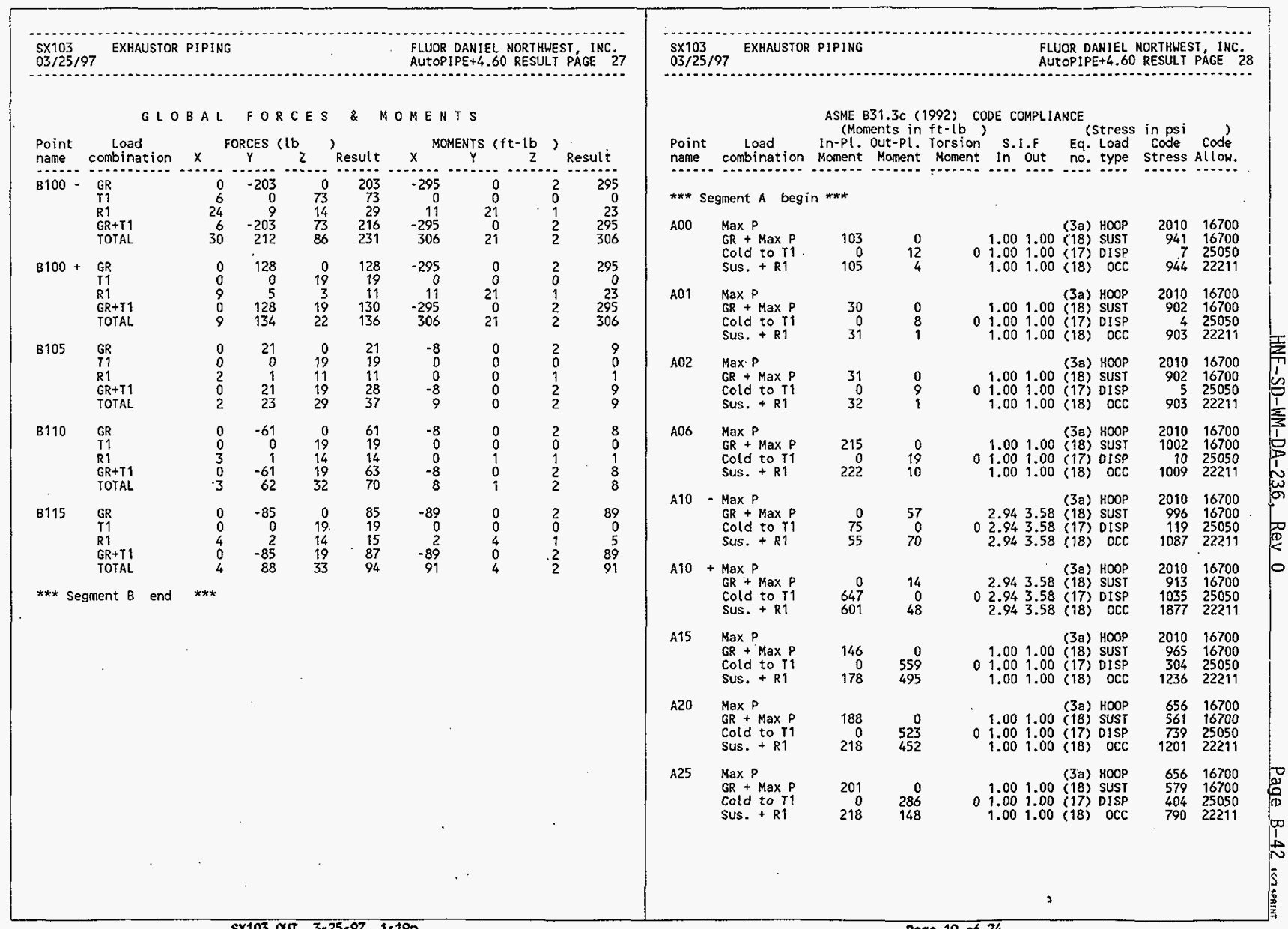




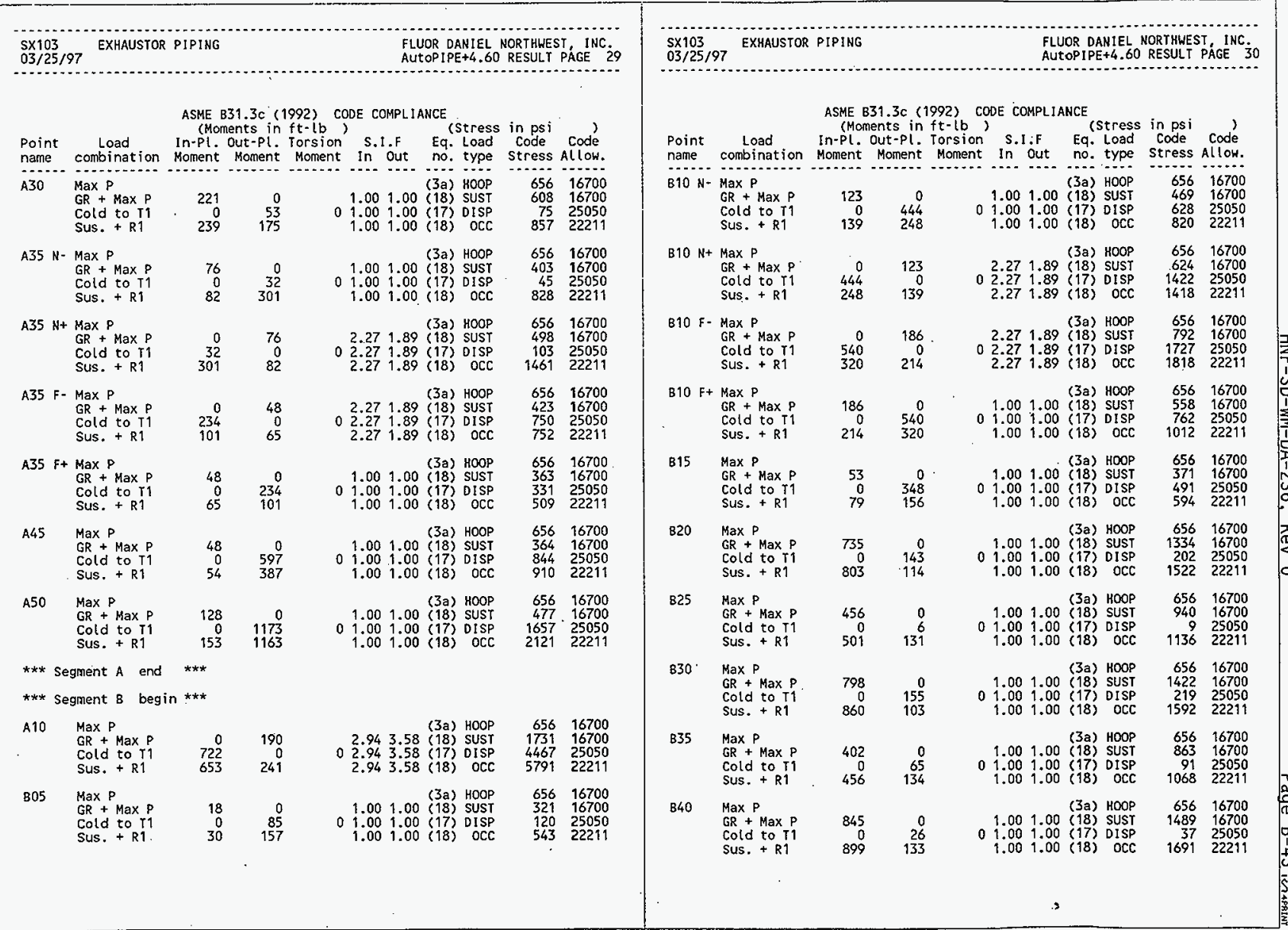




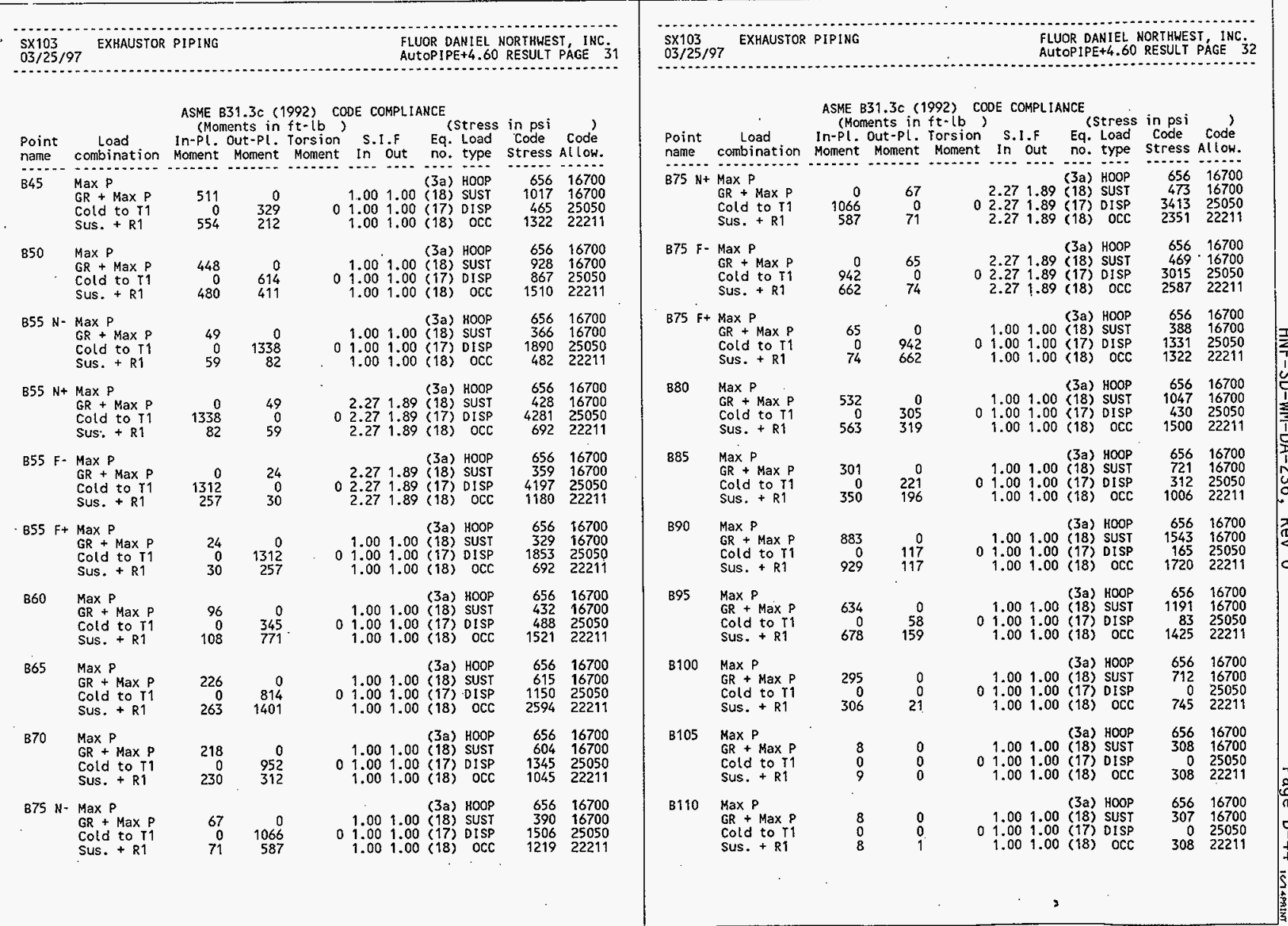




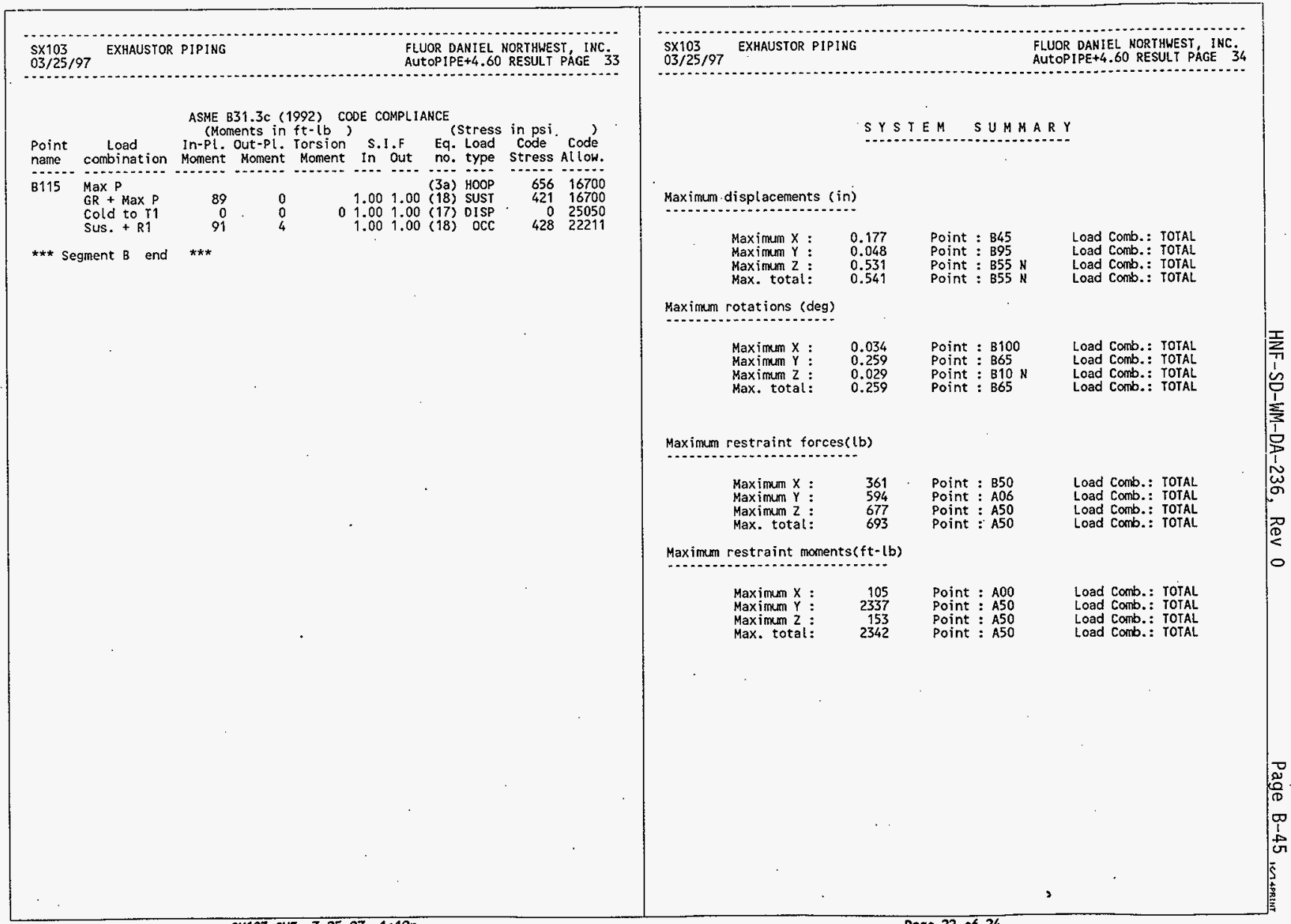




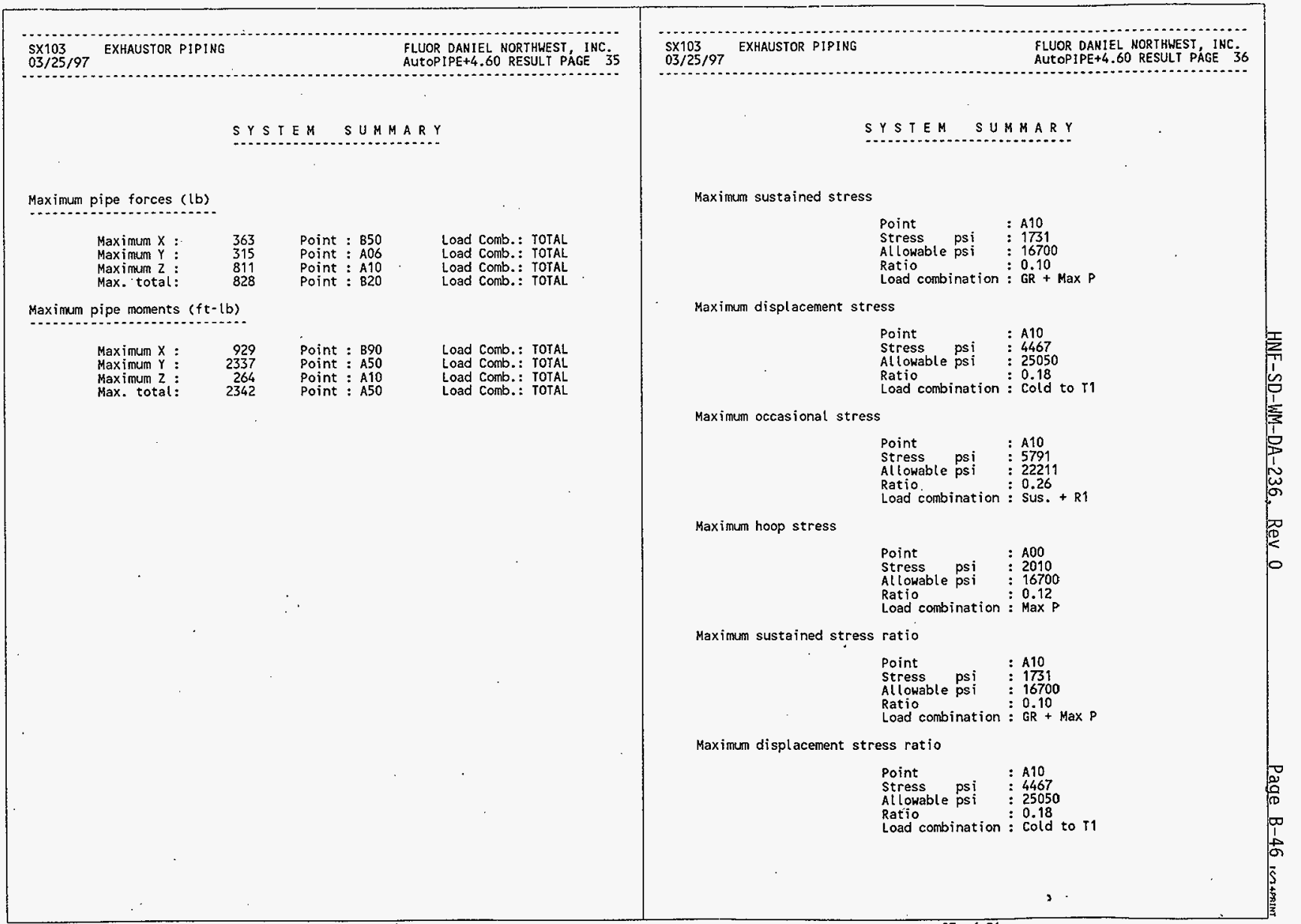




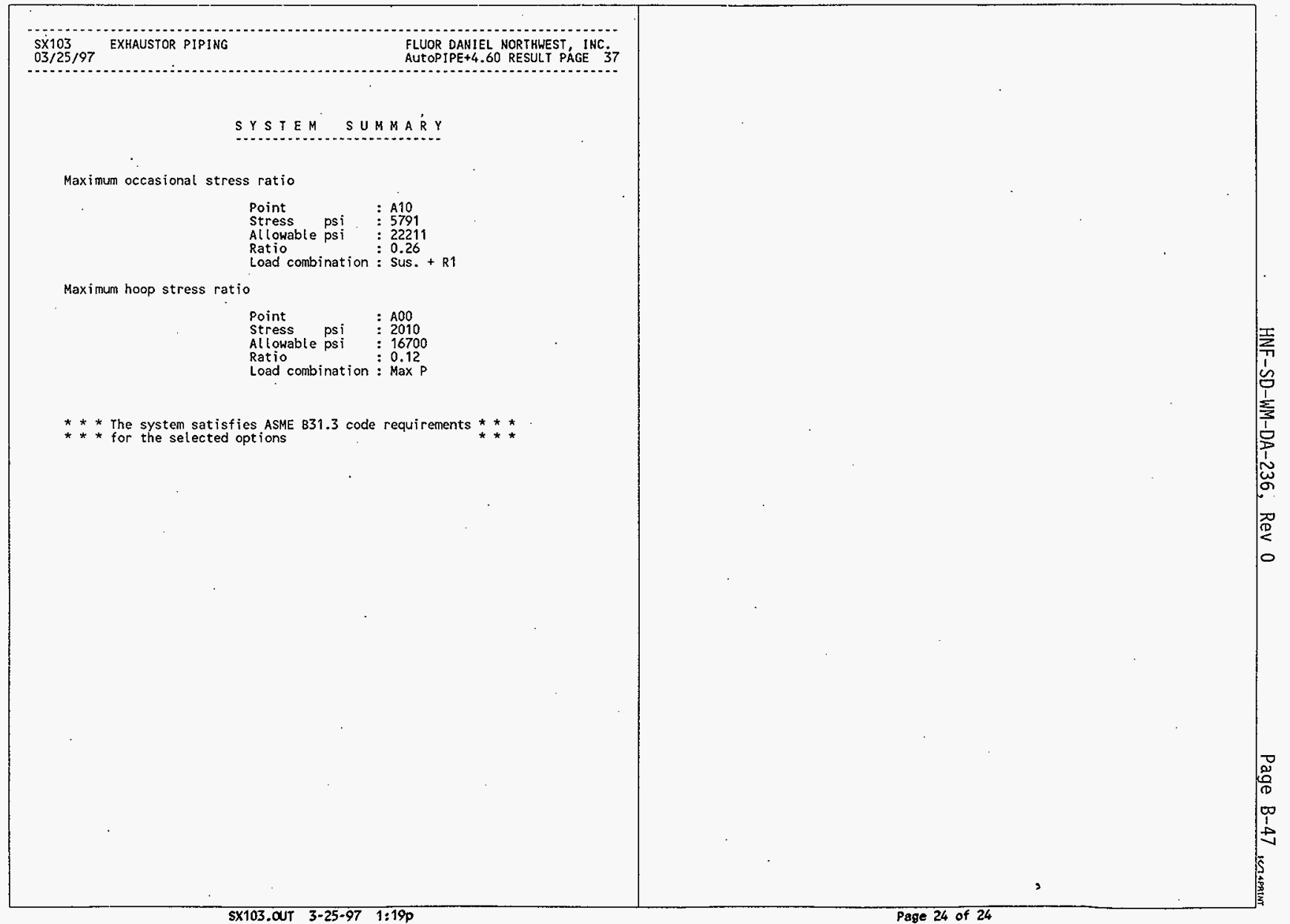




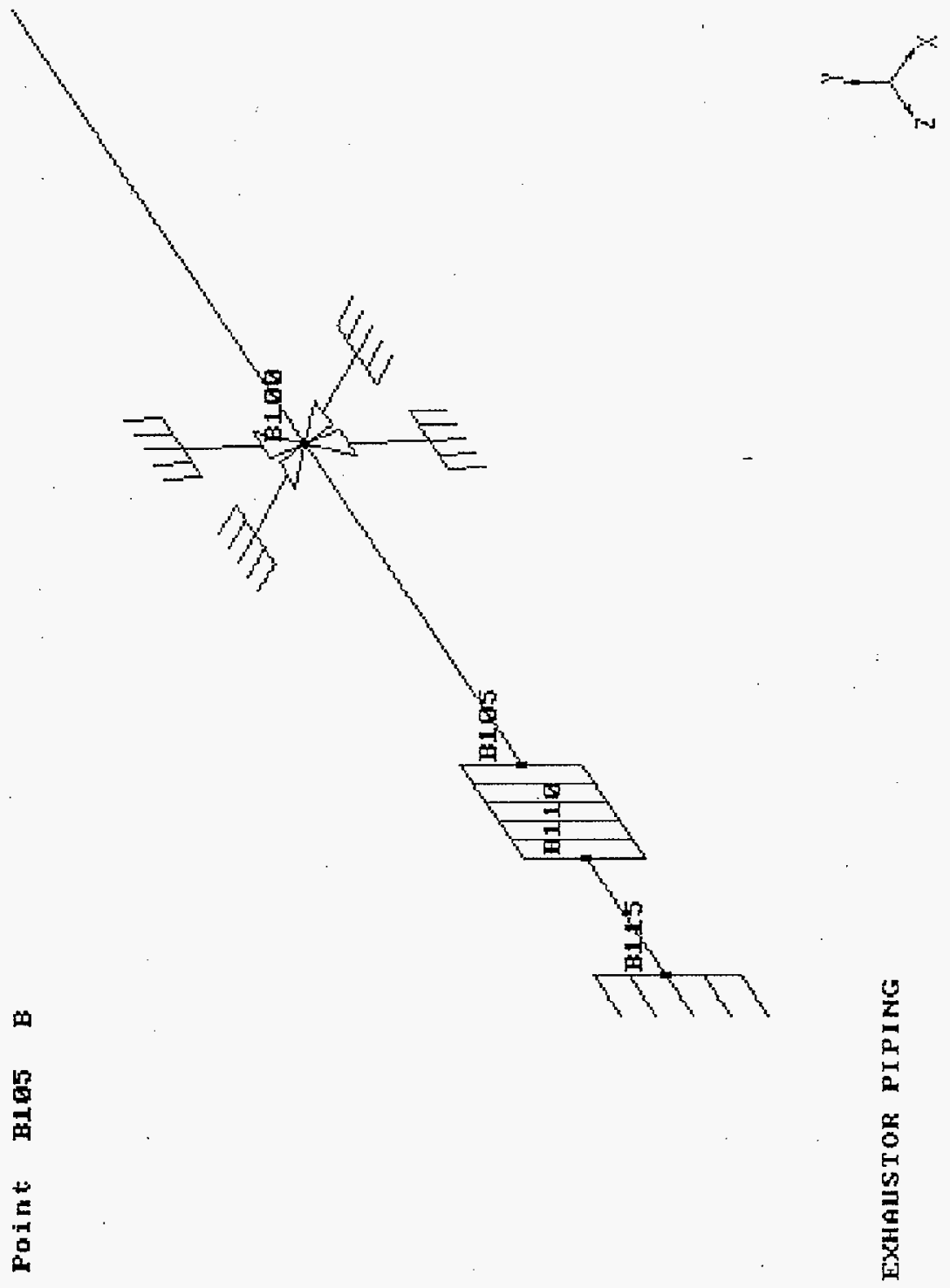



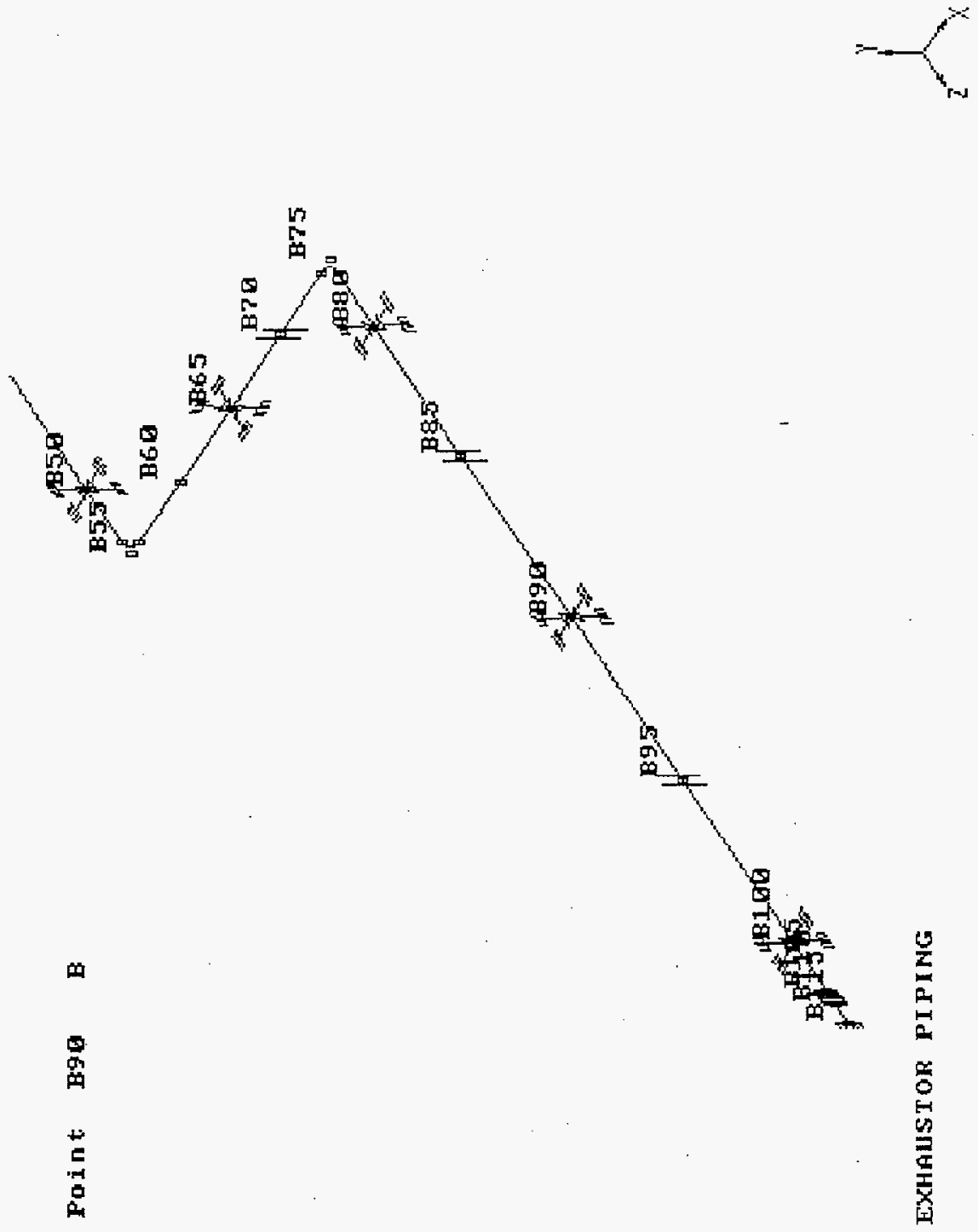


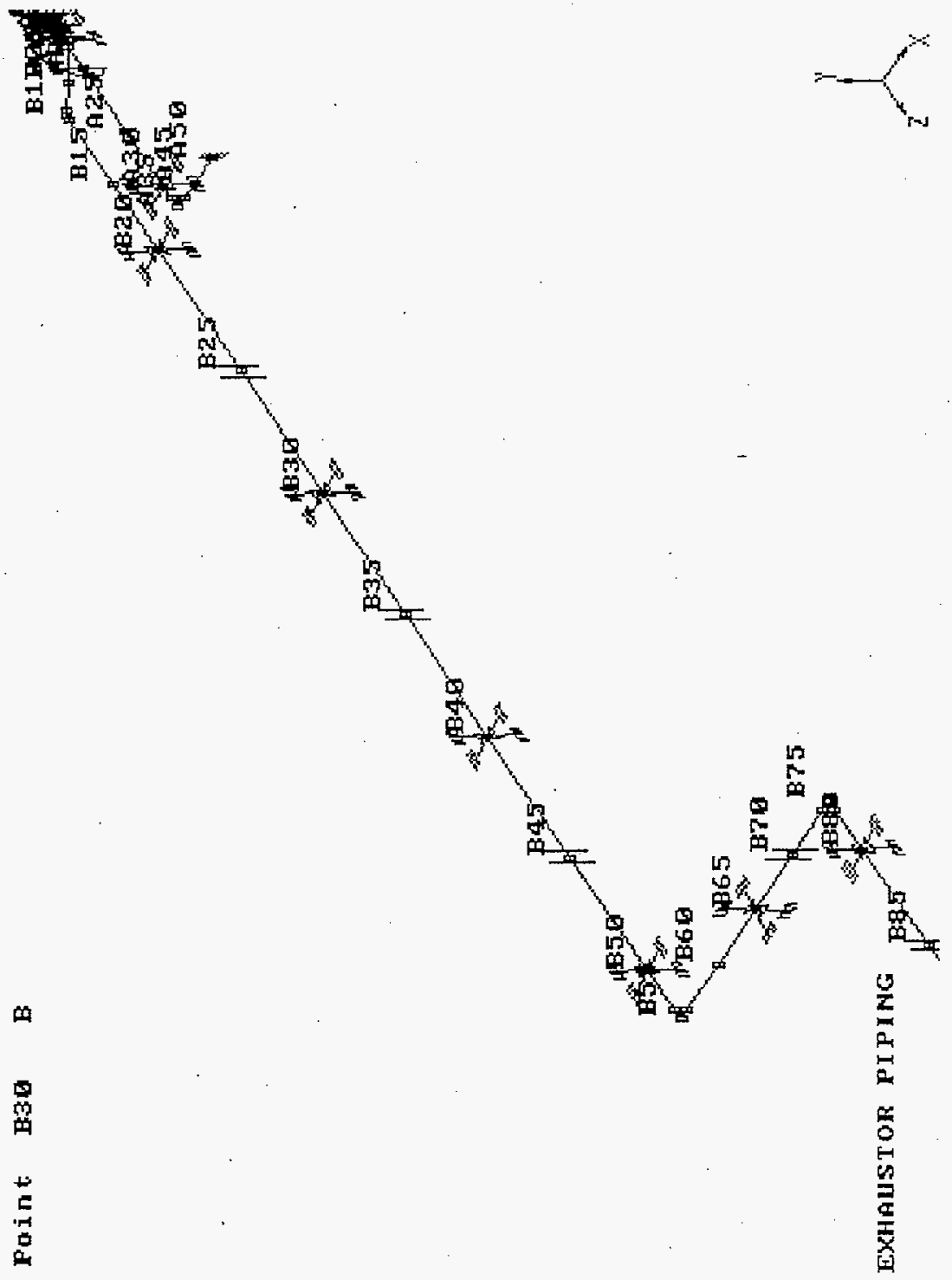



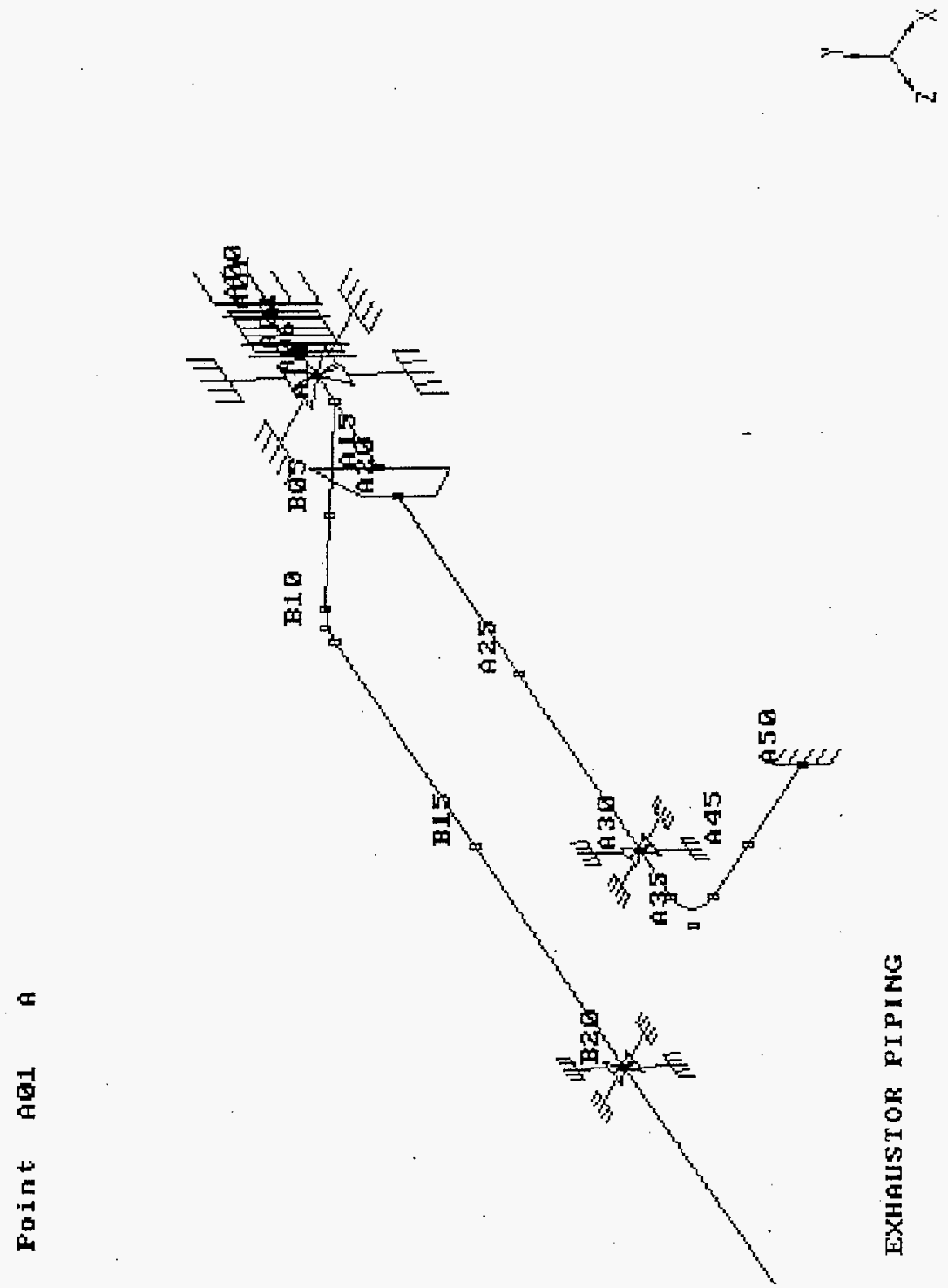
HNF-SD-WM-DA-236

Rev. 0

APPENDIX C: DESIGN CALCULATIONS FOR TANKS BY-105/106 INSTALLATION 


\section{Fluor Daniel Northwest}

\section{CALCULATION IDENTIFICATION AND INDEX}

This sheet shous the status and description of the attached Design Analysis sheets.
Discipline Mechanical
no/ Job Ho. POUXOO
Calculation No. $M-3$
Project No. \& Name 241-BY-105/106 500 CFM Exhauster Installation

Calculation Item

These calculations apply to:

Dwg. No. ECN 618793

Rev. Ho.

Dwg. No.

Rev. Ho.

other (study, CDR)

Rev. No.

The status of these calculations is:

[] Preliminary Calculations

[X] Final Calculations

[] Check Calculations con Calculation Dated )

[] Void Calculation (Reason Voided)

Incorporated in Final Drawings?

This calculation verified by independent "check" calculations? [] Yes [] No

Original and Revised Calculation Approvals:

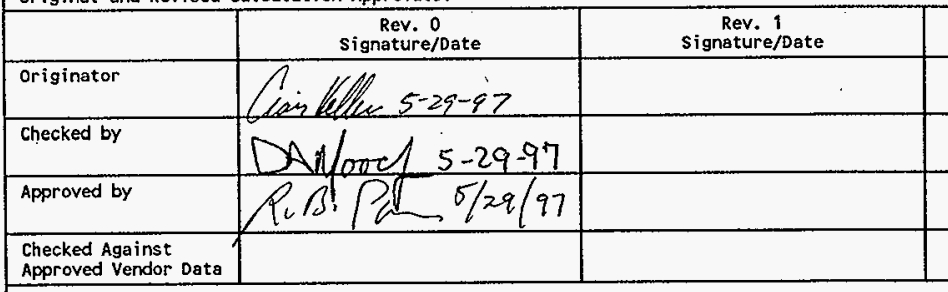

Rev. 2 Signature/Date

\begin{tabular}{|l|l|}
\hline \multicolumn{2}{|l|}{$\begin{array}{l}\text { Design Analysis } \\
\text { Page No. }\end{array}$} \\
\hline 3 & Analys is of Piping \\
\hline 3 & Analysis of Piping Support \\
\hline 5 & Exhauster Inlet Analys is \\
\hline 8 & Displacement at Expansion Joints \\
\hline 10 & Reactions at Tank Connections \\
\hline 14 & Missile Impact Analysis \\
\hline $18 / 4$ & Wind Loading Analysis \\
\hline $17 / 5$ & Ventilation Pressure Losses \\
\hline & Attachments \\
\hline & Miscellaneous Vendor and Analysis Information \\
\hline & Autopipe Analysis \\
\hline & \\
\hline
\end{tabular}


Fluor Daniel Northwest, Inc.

\section{DESIGN ANALYSIS}

client LMHC

Subject 241-BY-105/106 500 CFM Exhauster

Installation

Location 241-BY-105/106
Calc. No. $M-3$

Revision 0

Page No. 2 of 21

Wo/Job No. E40690

Date $4 / 14 / 97$

By C. M. Keller

checked 5/28/97

By D. A. Moody

Revised

By

\section{OBJECTIVE:}

Perform analysis on BY-105/106 exhauster installation (ECN 618793) to verify stresses are within acceptable values and to determine expected pressure drop in the ventilation ducting.

\section{CRITERIA:}

The exhauster and associated piping are analyzed using Safety Class 2 design loads provided in reference 1. Piping is analyzed using criteria specified in the ASME B31.3 Process Piping Code. Structural members have been analyzed using criteria specified in reference 3 .

\section{ASSUMPTIONS/GIVENS:}

Piping stresses and support reactions are contained in the piping analysis provided in the Attachments section. Analysis of the exhauster condensate piping in reference 6 is considered a governing case.

CALCULATION METHOD: Hand calculations and Autopipe analysis have been used to verify adequacy of the design.

\section{REFERENCES:}

1) Design of Expansion Anchors, GC-ANCR-01, Rev 1, ICF Kaiser Hanford Company

2) Minimum Design Loads for Buildings and other Structures ASCE 7-88, A.S.C.E.

3) Manual of Steel Construction, Allowable Stress Design, 9th Edition, American Institute of Steel Construction, 1989

4) Marks Standard Handbook for Mechanical Engineers, 9th edition, McGraw-Hi11

5) 1993 ASHRAE Handbook Fundamentals, American Society of Heating, Refrigeration and Air-Conditioning Engineers, Inc.

6) Installation Piping Analysis, Job \#E40690, Calculation M-2 Rev 0, C. M. Keller, 1997

7) Mechanical Engineering Design, 4th edition, Shigley and Mitchell, McGraw-Hill

8) Cameron Hydraulic Data, 16th Edition, Ingersol1-Rand, 1979.

9) Design Loads for facilities, GC-LOAD-01, Rev 1, ICF Kaiser Hanford Company

10) Installation Piping Analysis, Job \#P4H514, Calculation M-1 Rev 1, B. M. Koons, $4 / 30 / 96$ 


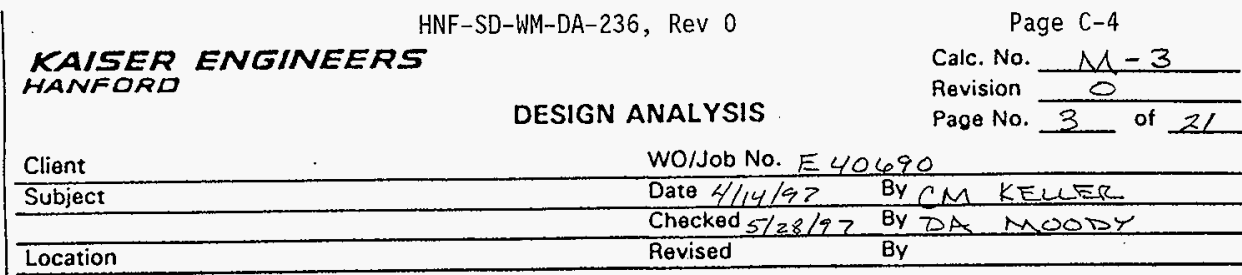

1) ATTACHED AUTOPIPE ANALYSIS DETERMINED PIPING STRESSES TO BE WITHIN ALLOWABLE PER ASHE BB/,3 PROCESS PIPING CODE.

2) ANALYZE PIDE SUTOORTS USINL REACTION FORCES FROM AUTOPIDE ANALYSIS AND WIND LOADING DETERMINES IN CALCULATION $M-Z$,

WORST CASE HORIZONTAL LOADING IS SUPPORT BG5. MAXIMUM COADING occurS DUE TO GRAVITY, THERMAL AND WIND LOADS.

VERTICAL SOU \#

HORIZONTAL SO (PARALLEL TO PIPERUN)

HORIZONTAL $16 Z^{\text {* }}$ (PERTENDICULARTO PIPERUN)

Horiz, wins $344 *$

CHECK OVERTURNING OF SUPPORT

ELEVATION AT PIPE $\mathcal{E}$ IS:

$$
653.10^{\prime}-\frac{\left(28^{\prime}+27.7^{\prime}\right)\left(1 / 8^{\prime \prime} \frac{\text { SIDE }}{f L}\right)}{12}=652.5^{\prime}
$$

AVG GRADE ELEVATION $15: 1 / 2\left(648^{\prime}+649^{\prime}\right)=648.5^{\prime}$

DISTANCE FROM GRAPE TO PIPE \& IS:

$$
\left(652.5^{\prime}-648.5^{\prime}\right) 12=48^{\prime \prime}
$$

54-4300-037 KEH-0037.00 (06/92) 


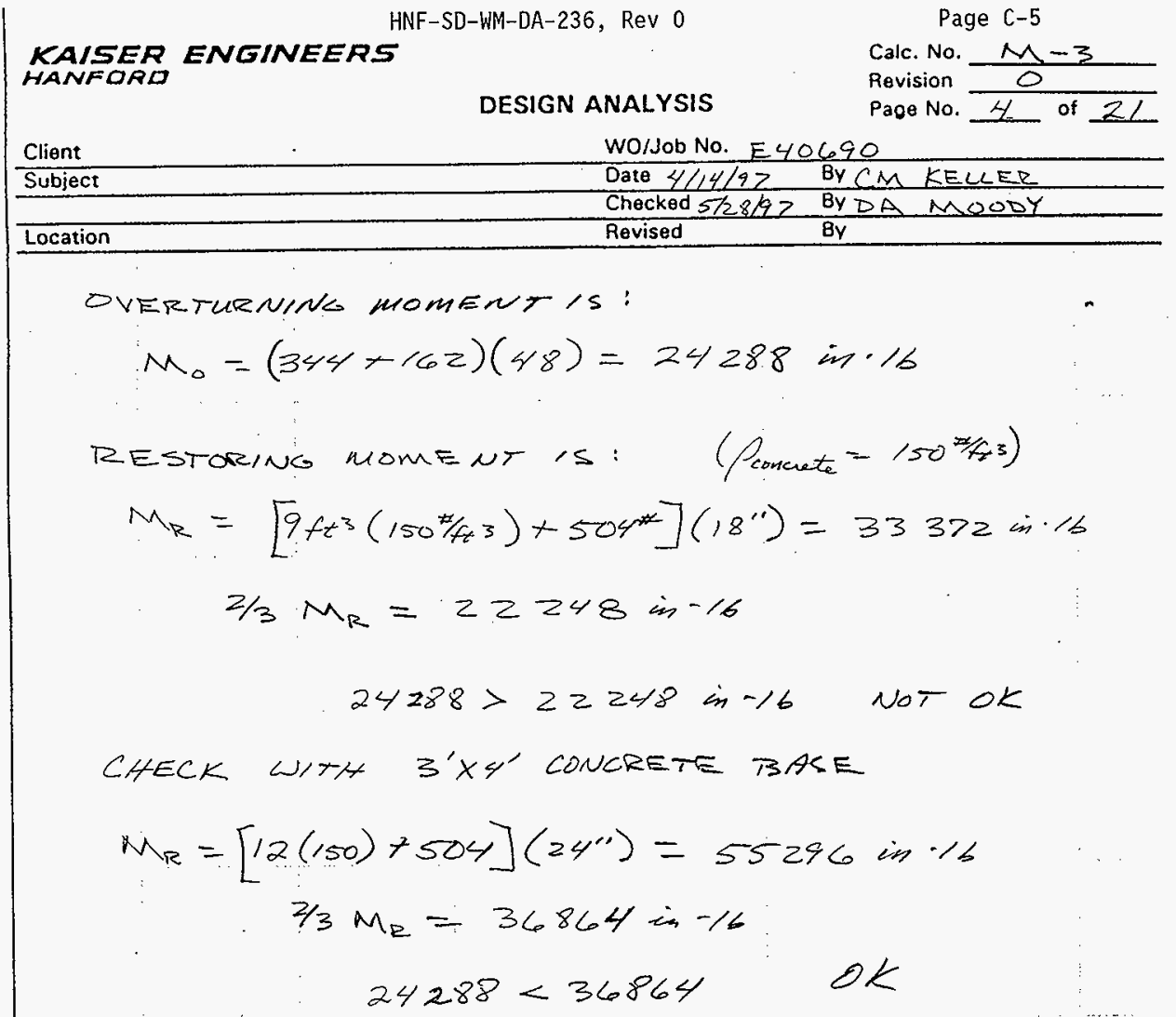

$\therefore$ Concrete base for anchors NeEdS to be INCREASED TO: $3^{\prime} X Y^{\prime}$ FOR $A=Z / 3$ SAFETY MARG For overtuRnaker,

3) MAXIMUM BENDINL MOMENT ON THE PIPE SUPPORT IS ONLY SLIGHTLY (rIO\%) GREATER THEN USED IN SUPPORT ANALYSIS FOR CALCULATION MIL, MARGIN OF SAFETY IN THAT ANALYSIS JUSTIFIES NOT ANALYZNG THE PIPE SUPPORTS FOR THIS INSTALLATION. SUPPORT DESIGN WAS DETERMINED ADEQUATE IN CALCULATION $M-2$.

54.4300.037 KEH.0037.00(06/92) 
HNF-SD-WM-DA-236, Rev 0

\section{KAISER ENGINEERS}

\section{HANFORD}

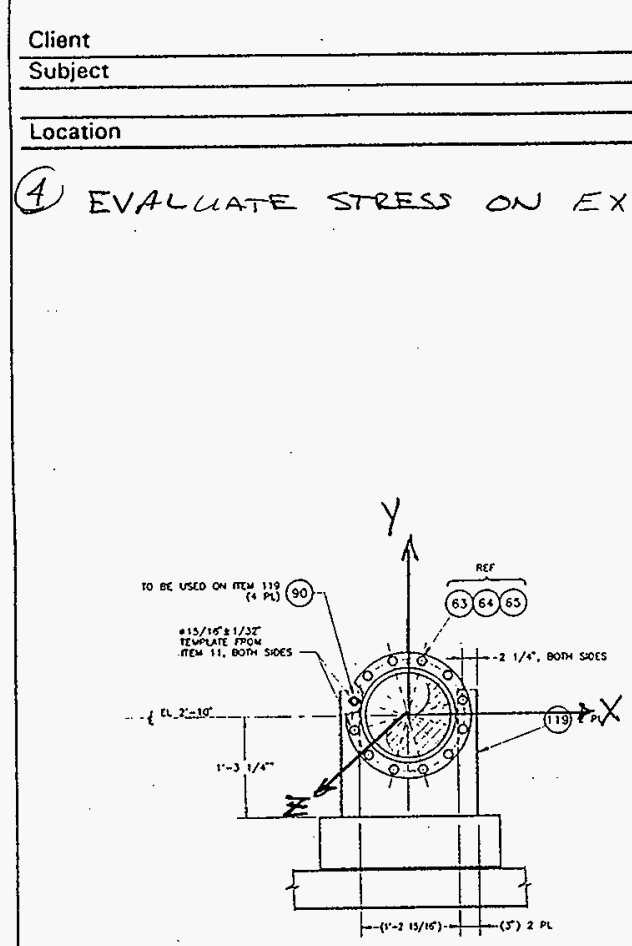

\section{Client}

Subject

DESIGN ANALYSIS

\section{Revised}

(4) EVALLLATE STRESS ON EXHAM
Page $C-6$

Calc. No. $\wedge-3$

Revision $O$

Page No. 5 of 21

Wo/Job No. E40\%90

Date $4 / 14 / 9 Z$ BYCRAK MIKELLER
Checked $s / 28 / \mathrm{s}$ By toA MOODL

By

(1)




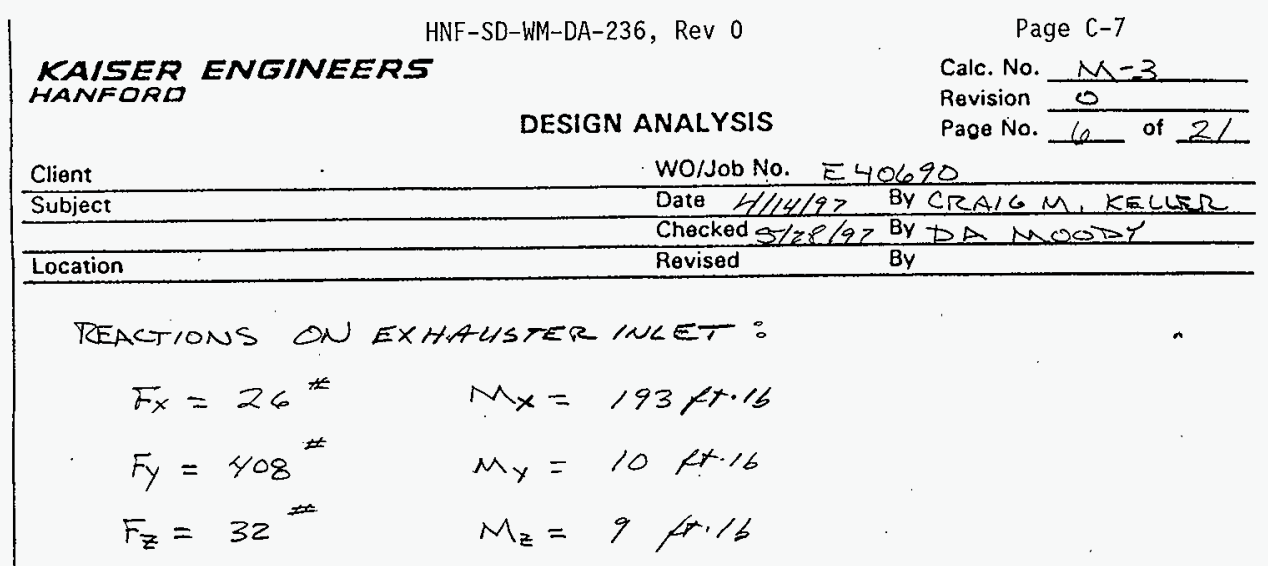

REACTIONS ON INLET SUPPORT ARE AS FOLLOWS:

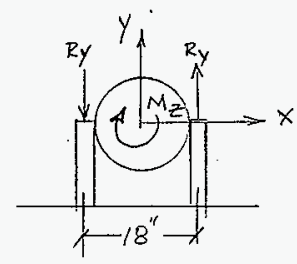

REACTION DUE TO $M_{Z}$ :

$$
9 A^{2} \cdot 16=2 R_{y}(9 / 12) \Rightarrow R_{y}=6^{*}
$$

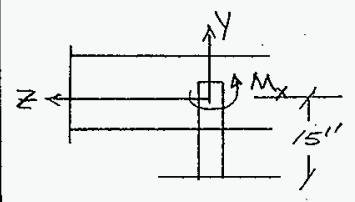

REACTION DUE TO $M_{X}$ :

$$
193 f+16=2 R_{x}\left(\text { '. }^{\prime \prime}\right) \Rightarrow R_{z}=77.2
$$

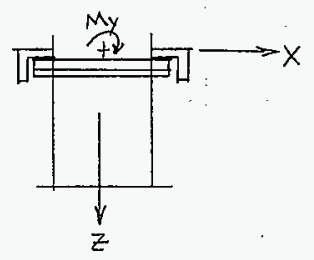

ReAction DuE to My:

$$
10 A^{x} \cdot 16=2 R_{E}\left(9 / I^{\prime \prime}\right) \Rightarrow R_{z}=6.7^{2 *}
$$

TOTAL SWTPOET FORCES:

$$
\begin{aligned}
& F_{x}=26 * \\
& F_{y}=408^{*}+6^{*}=414^{*} \\
& F_{z}=32^{*}+77.2+6.7^{* 4}=116^{*}
\end{aligned}
$$

54.4300.037 КEH-0037.00 106/921 


$$
\begin{aligned}
& \text { HNF-SD-WM-DA-236, Rev } 0 \\
& \text { Page } C-8 \\
& \text { KAISER ENGINEERS } \\
& \text { DESIGN ANALYSIS } \\
& \text { Call. No. } M-3 \\
& \text { Revision } 0 \\
& \text { Page No. } 7 \text { of } 21 \\
& \text { Subject } \\
& \text { Location } \\
& \text { WO/Job No. E40690 } \\
& \text { Date 4-14-97 By CRA/C M. KFLLEC } \\
& \text { Cracked } 5-28-97 \text { BY DA MOOSe } \\
& \text { Revised } \\
& \text { BY } \\
& \frac{\Delta_{a}}{s_{a}}+\frac{\sigma_{b x}}{s_{b x}}+\frac{\Delta_{b x}}{s_{b_{z}}} \leq 1 \text { eff } 3 \text { eq HI-3 } \\
& \sigma_{b x}=\text { Bending stress in } x \text {-dir } \\
& \sigma_{b z}=\text { Bending Stress in } z \text {-dir } \\
& S_{a}, S b_{x}, S b_{z}=\text { ALCOWHBLE STRESSES }=22 \mathrm{kS} .^{\circ} \\
& \Delta_{a}=\frac{F_{y}}{A}=\frac{414^{* 4}}{1.19 \mathrm{im}^{2}}=348 \mathrm{js}^{\circ} \\
& \Delta_{b x}=\frac{F_{x}\left(15^{\prime \prime}\right)}{S_{x x}}=\frac{26(15)}{.260}=1500 p^{\prime} \\
& \sigma_{b z}=\frac{F_{z}\left(15^{\prime \prime}\right)}{S_{z z}}=\frac{116(15)}{.542}=3210 \mathrm{ps} \\
& \frac{348+1500+3210}{22000}=.23<10 K
\end{aligned}
$$

$54-4300-037 \quad$ KEH-0037.00 (06/92) 


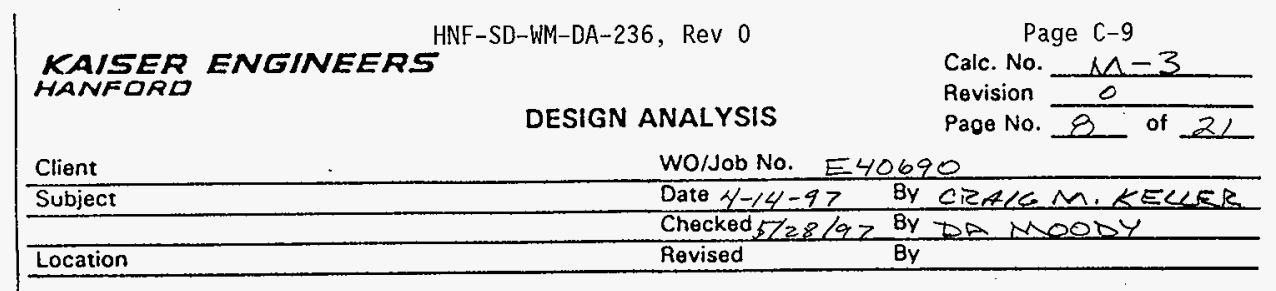

(5) CHECK DISPLACEMENT AT EXPANSION TONT LUCATION'S:

QIZ" EXPANSION SOINT, PATHWAY RCSFFSOBC-MI

ALLOWABE TDISPLACEMENTS:

AXIAL: $3.28^{\prime \prime}$
LATERAL: $0.55^{\prime \prime}$
ANGULAR: $\quad 10^{\circ}$
TORSION: $.055^{\circ}$

CALCULATED DISPLACEMENT POINTHOZ IN PIPINC QMALYSIS

TRANSCATION: ROTHTION:

$$
\begin{array}{ll}
X-.009^{\prime \prime} \text { (LATERAC) } & X-.086^{\circ} \text { (ANGULAR) } \\
Y-.059^{\prime \prime} \text { (LATERAL) } & Y-.035^{\circ} \text { (ANGULAR) } \\
Z-.05 \text { A }^{\prime} \text { (AXIAL) } & Z-.002^{\circ} \text { (TORSION) }
\end{array}
$$

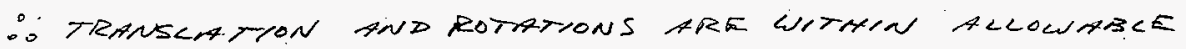
VALUES FOR $\phi I L^{\prime \prime}$ EXPANSION JOMNT AT EXHAUSTER INLET.

Q6" EXPANSION TOINT, PAYHWOY GCSFF SOIZC-MI

ALCOCUABCE DISPLACEMENTS:

Axial: $2.46^{\prime \prime}$

LATERAL: .84"

angular: $10^{\circ}$

TORSION: $.082^{\circ}$

$54-4300-037 \quad \mathrm{KEH}-0037.00(06 / 92)$ 
KAISER ENGINEERS

HANFORD

Client

Subject

Location
HNF-SD-WM-DA-236, Rev 0

\section{DESIGN ANALYSIS}

Page $C-10$

Calc. No.

Revision

Page No.

$\frac{M-3}{9}$ of 21

WO/Job No. E40690

Date $4-14-97$ BY CRALCOMUKELLES
Checked $5-28-97$ By $D A N 000 \%$ Revised By

CALCULATED DISPCACS MENTS AT POINT C7O (BYHOS INLET):

$$
\begin{aligned}
& \text { TRANSLATHON } \\
& X \quad .033^{\circ} \text { (LATERAL) } \\
& Y \quad .005^{\prime \prime}(\angle A T E R A L) \\
& Z \quad .155^{\prime \prime}(A X I A L)
\end{aligned}
$$$$
\text { Roratron }
$$$$
.010^{\circ} \text { (ANGULAR) }
$$$$
.016^{\circ} \text { (ANGUCAR) }
$$$$
.005^{\circ} \quad(\text { TORSION) }
$$

CALCULATED DISPLACEMENTS AT POINT 3175 (BY-106 MLET)

Prenas

$\times .314-\angle .222$ LATERAL

$Y .089$ (LATERAC)

$z$
.177<smiles>C1C[As]2CCC[As]2C1</smiles>

LATERAL

AXIAL

\section{$72079270 \mathrm{~N}$}

$.196^{\circ}$ (ANCtroRs)

$.098^{\circ}$ (angucar)

$.02 z^{\circ}$ (ANC troRs)

$\therefore$ TRANSLATPON ANTS ROTATIONS ARE WITHIN ALCOUALE. VALUES FOR $\phi \zeta^{\prime \prime}$ EXPANSION JOINTS AT BOTA LOCATHNS, 
HNF-SD-WM-DA-236, Rev 0

\section{KAISER ENGINEERS HANFORD}

\section{DESIGN ANALYSIS}

Client

Subject

Wo/Job No. E40690

\section{Location} Date $4-14-97$ By CRA/G M, KELER Checked $5-28-97$ By $D A$ NoODY Rovised By

Calc. No. $\mathrm{N}-3$

Revision 0

Page No. 11 of 21

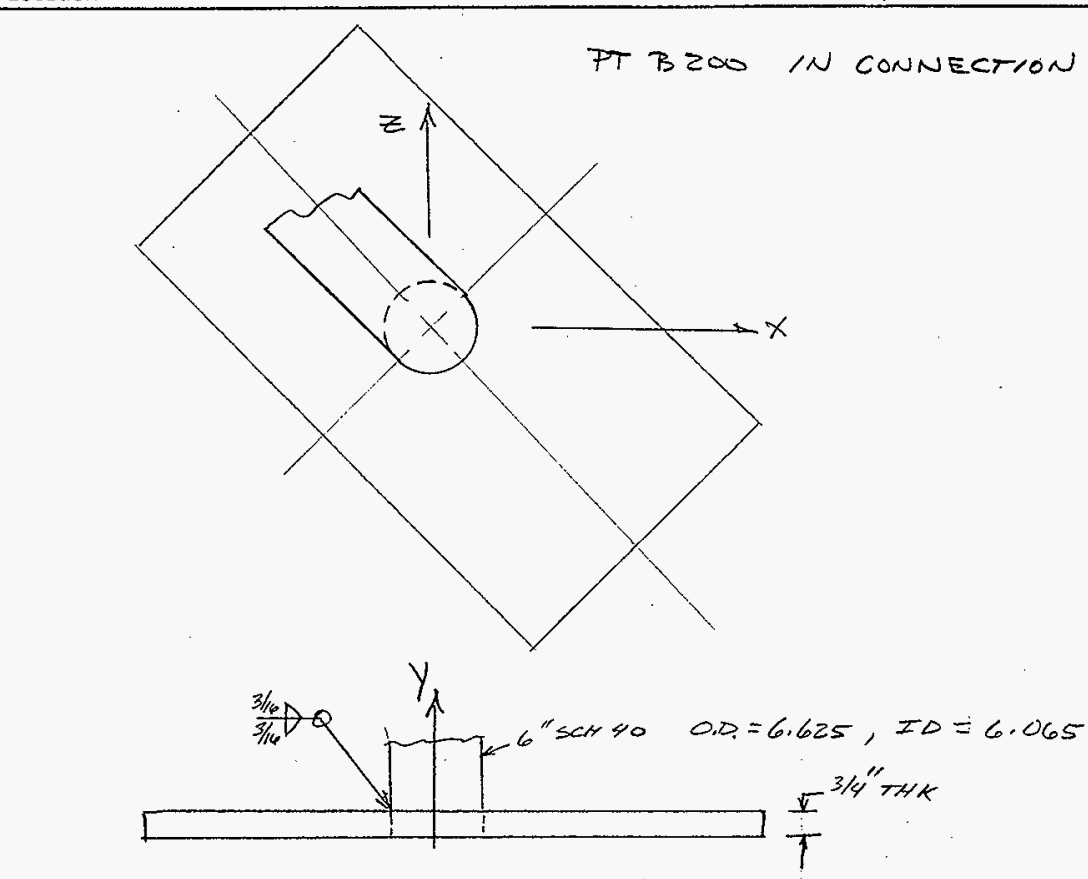

WELD LOADING

$F X=108^{2}$. HORIZONTAC SHEAR

$F_{Y}=908^{\#}$ NORMAL STRESS

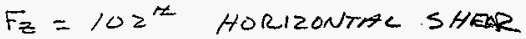

$M_{x}=935 \mathrm{ft} / \mathrm{l}$. Bending sreess

$M_{Y}=119 \mathrm{fH}^{\prime} / 6$ TORSION (PEODUCES SHEAR)

$M_{z}=827+18$ Bend.2q STRESS 


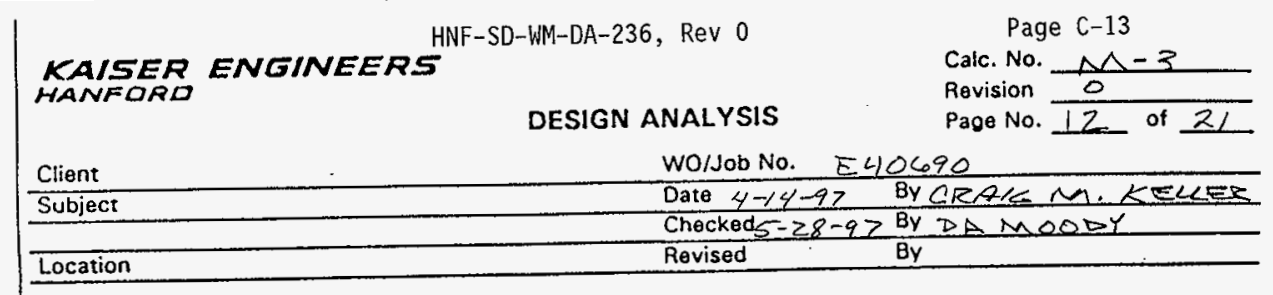

ALLOWABLE WELD STRESS IS SAMEAS PITE

pen ASME B31.3 $\sigma_{a 11}=16.7 \mathrm{ks}$

$$
\begin{aligned}
\text { WELD AREA } & =(707 h \pi D) 2 \text { BOM WELDS } \\
& =(707)(.188)(\pi)(6.625)(2)=5.53 \mathrm{in}^{2}
\end{aligned}
$$

Shean stress dweto torsion Loto My: ef 7 sect $9-3$

$$
\begin{aligned}
& T=\frac{M r}{J}
\end{aligned}
$$

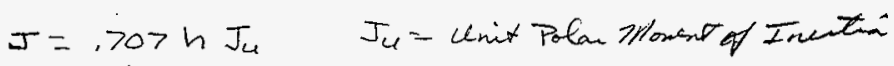

$$
\begin{aligned}
& J_{u}=2 \pi r^{3} \\
& J=.70>(.188)(2 \pi)(3.31 \mathrm{im})^{3}=30.29 \mathrm{in}^{\prime \prime} \\
& J_{\text {rorki }}=z(30,29)=60,57 \text { in } 4 \text { for sooth ehelds } \\
& T^{2}=\frac{119 \mathrm{ft} .16(12 \mathrm{im} / \mathrm{ft})\left(3.31^{\prime \prime}\right)}{60.57 \mathrm{in} 4}=178 \mathrm{psi}^{\circ}
\end{aligned}
$$

Shea stars dere to show Facas $F_{x} \neq F_{z}$ :

$$
\begin{aligned}
& p^{\prime}=\frac{F}{A}=\frac{\left(108^{2}+102^{2}\right)^{1 / 2}}{5.53 \mathrm{in}^{2}}=27 p s i \\
& \text { TothL sheon } \frac{T}{70 \%}=78+27=105 p \mathrm{~s}^{\circ}
\end{aligned}
$$

54-4300-037 KEH-0037.00 (06/92) 


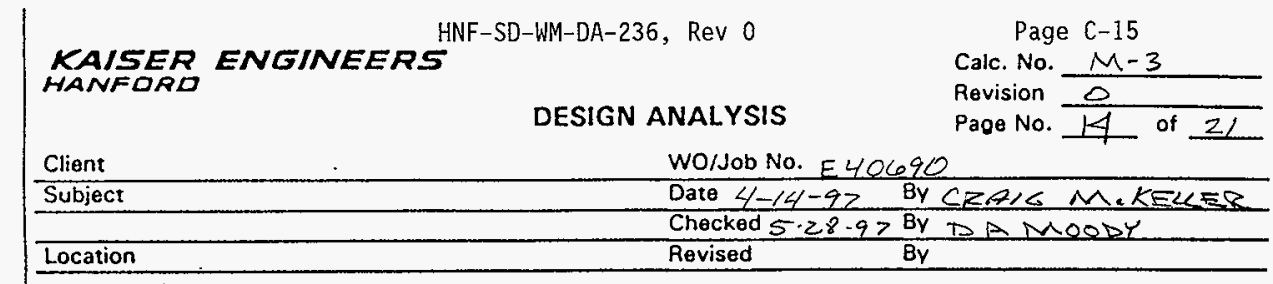

Maximum plate stress $S_{m}=k \frac{w R^{2}}{t^{2}}$ y y pqs-5iz

$$
\begin{aligned}
& R / r=\frac{60}{42}=1.4 \Rightarrow K=.487 \quad \text { T.614 } 5.2 .20
\end{aligned}
$$

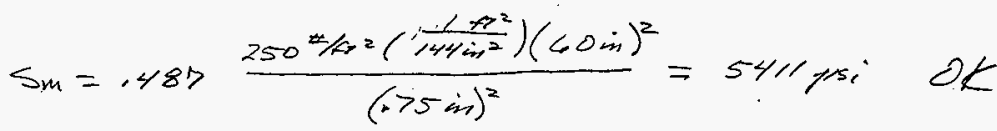

$\therefore$ Plate HICKNESS IS ADEQUATE.

(9) Analysis for missiLE ZMPACT AND WIND LOADING ON THE Ventharion DuCT WORK HAS previously beer performed in CALCULATION M-2. SATSNACTORY ReSULTS iN HAN

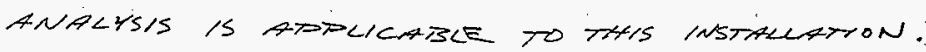

54-4300.037 KEH-0037.00 (06/92) 
HNF-SD-WM-DA-236, Rev 0

Page $\mathrm{C}-16$

ANALYTICAL CALCULATIONS

Page 15 of 21

Subject Cate $M-3$

JOB E40690

originator AF Weller Date $4-2-97$

Checker C.M. KELLER

Date $4-14-97$

(12) Calculate expected pressure from atmosphere to exhausted inlet for both nuns of ductwork.

Use Saturated air at $140^{\circ} \mathrm{F}$.

Use $Q=250$ CFM per duct run.

Assume I"wg pressure drop for inlet filter

Determine $\triangle P$ for the following:

inlet HEPA filter and riser (both tanks)

2) Outlet riser and tee connection (BY-105)

3) Ductwork riser to exhauster - long run (BY-106)

4) Ductwork riser to exhauster - short run (By-105)

5) Exhausted inlet fitting

6) Outlet riser (By-106)

1) Inlet filter $\$$ riser (Typical arrangement)

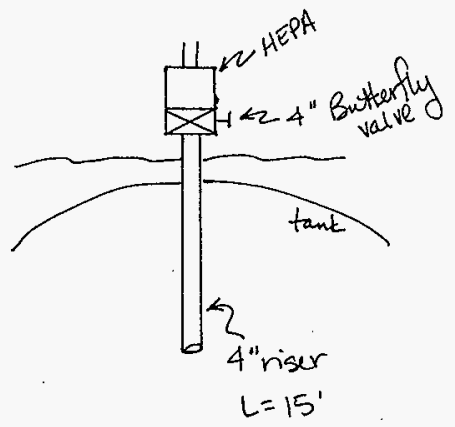

Hep filter $\Delta p=1 " w G$

Equivalent length method

$\left.\begin{array}{l}\text { Riser } L=15^{\prime} \\ \text { Entrance loss } L_{e}=20^{\prime}\end{array}\right\}$ Ref.4

4" Butter fy valve $L_{e}=15.1^{\prime}$ Ref. 8

$L_{e_{\text {inlet }}}=15+20+15.1=50.1^{\prime}$

BD-6400-060.1 (07/93) 
HNF-SD-WM-DA-236, Rev 0

Page $\mathrm{C}-17$

ANALYTICAL CALCULATIONS

subject $\mathrm{Ca} C M-3$

Originator A.F. Weller

Checker C.N. KELLER
TOB E40690

Page 16 of 21
Date $4-2-97$

Date $4-14-97$

2) Outlet Riser \& tee connection tank BY -105

Assume loss for side outlet on a $12 \times 6$ reducing tee is equivalent to a $1 z^{\prime \prime}$ side outlet tee followed by a $12 \times 6$ contraction

Use equivalent feet method: Exit loss $L_{e}=47^{\prime}$ $12^{\prime \prime}$ riser $L=15^{\prime}$ $12^{\prime \prime}$ side out tee $L_{e}=60^{\prime}$ $12^{\prime \prime} \times 6$ "contraction $L_{e}=11$ "

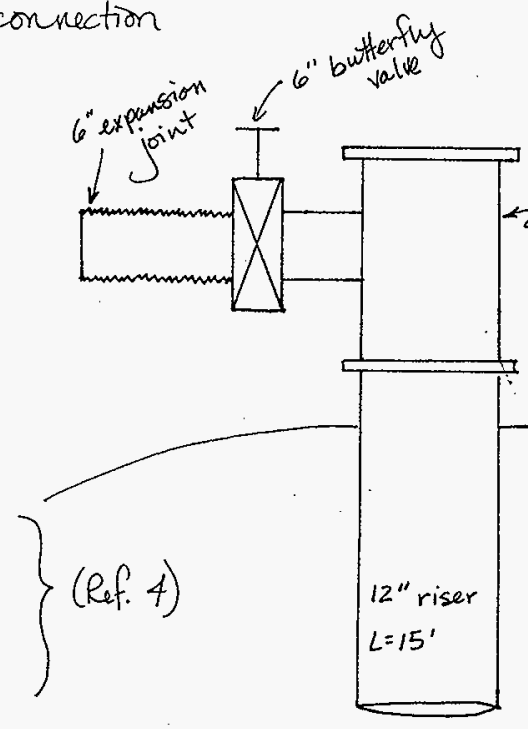

6" Butterfly valve $L_{e}=22.7^{\prime}$ (Ref. 8)

$6^{\prime \prime}$ Expansion joint $L_{e}=8^{\prime}$ estimated

Total $L_{e_{12^{\prime \prime} \text { riser }}}=47+15+60+11=133^{\prime}$

4) Duct work (BY-105) [Note 3) follows on next page, out of sequence] 26 " $45^{\circ}$ el lows $\quad L_{e}=8^{\prime}$ each

$6 "$ pipe

$$
L=7^{\prime} 3.75^{\prime \prime}+10^{\prime}+8.6=25.9^{\prime}
$$

conservative

6" butterfly value

6" expansion joint $L_{e}=22.7^{\prime}$

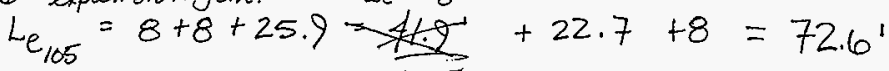

BD-6400-060.1(07/93) 
HNF-SD-WM-DA-236, Rev 0

Page $C-18$

ANALYTICAL CALCULATIONS

Page $; 1 Z$ of $2 \%$

Subject $\mathrm{CaLC} M-3$

Originator A.F. Weller

Checker C.M. KELLER
TOB EU 4.90

Date $4-2-97$

Date $4-14-8\rangle$

3) Duct work - long run (B Y-106)

$366^{\prime \prime} 45^{\circ}$ elbow $L_{e}=8^{\prime}$ each

$26^{\prime \prime} 90^{\circ}$ long radius elbow $L_{e}=10^{\prime}$ each

6 " pipe

$$
\begin{aligned}
L= & 59^{\prime} 2.5^{\prime \prime}+28^{\prime}+33^{\prime} 8.5^{\prime \prime}+10^{\prime} \\
& +12.6^{\prime}+11.1^{\prime} \\
= & 154.6^{\prime}
\end{aligned}
$$

$L_{C_{106}}=8+8+8+10+10+154.6=198.6^{\prime}$

6) Outlet riser (By-106)

Air exits the tank through a 24 "pipe. The pipe is run is short and exits into a condenser pit. This $p_{1}^{i t}$ is $3: \times 6^{\prime} \times 12^{\prime}$ (approx).

Assume the losses are negliable in this area.

$6^{\prime \prime}$ exit $L_{e}=25^{\prime}$

$6^{\prime \prime} 90^{\circ}$ LR. elbow $L_{e}=10^{\prime}>\operatorname{Ref} .4$

6 "btrfly value $L_{e}=22.7^{\prime}$ Ref. 8

6 "exp .joint $L_{e}=8^{\prime}$ estimated

Total $L_{e_{\text {low out }}}=25+10+22.7+8$

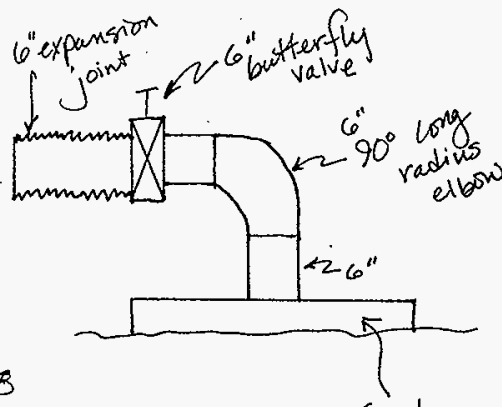

$=65.7^{\prime}$

condenser

pit

BD -6400-060.1 (07/93) 
HNF-SD-WM-DA-236, Rev 0

Page $C-19$

ANALYTICAL CALCULATIONS

Page 18 of 21

Subject $\mathrm{CalC} M-3$ TOB $E 40690$

Originator A.F. Weller

Date $4-2-97$

Checker L.M. KELLER

Date $4-14-97$

5) Exhausted inlet fitting

Use Ref. 5

Use ED 5-10 Symmetrical

Wye, $60^{\circ}$, with reduction

$$
\begin{aligned}
& A_{b_{1}}=A_{b_{2}}=\pi r^{2}=\pi 3^{2}=28.3 \mathrm{in}^{2} \\
& A_{c}=\pi 6^{2}=113 \mathrm{in}^{2} \\
& \frac{A_{b_{1}}}{A_{c}}=\frac{A_{b_{2}}}{A_{c}}=.25 \text { round to } .3 \\
& Q_{b_{1}}=Q_{b_{2}}=250 \mathrm{~cm} \\
& Q_{c}=500 \mathrm{cfm} \\
& \frac{Q_{b_{1}}}{Q_{c}}=\frac{Q_{b_{2}}}{Q_{c}}=.5 \\
& C_{b_{1}}=0.86 \\
& C_{b_{2}}=0.86
\end{aligned}
$$

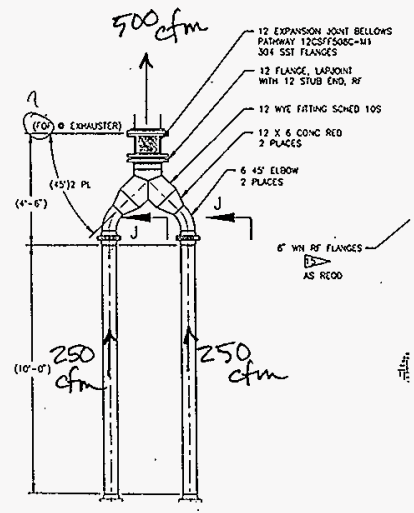

Calculate loss: $\Delta p=c p_{\nu}$

$p \cdot 32.6$, eq 29

$\Delta p=$ pressure loss (in.W.G.)

$C=$ loss coefficient

$P_{v}=$ velocity pressure (in. W.G.)

$$
\begin{array}{rl}
P_{v}=\rho\left(\frac{V}{1097}\right)^{2} & V=\text { velocity }\left(f_{\rho m}\right) \\
\rho & =\text { air density }\left(16 \mathrm{~m} / \mathrm{ft}^{3}\right)
\end{array}
$$

For saturated air @140. F $\rho=.0613 \mathrm{lbm} / \mathrm{ft}^{3} \quad$ Ref. 6

$V=.1246 \mathrm{ft} / \mathrm{min}$ for $250 \mathrm{cfm}$ in $6^{\prime \prime}$ sch. 40 pipe $V=\frac{Q}{A}$

8D-6400-060.1 (07/93) 
HNF-SD-WM-DA-236, Rev 0

Page $\mathrm{C}-20$

ANALYTICAL CALCULATIONS

Page 79 of 21

Subject $\mathrm{Calc} M-3$

Originator A.F. Weller

Checker C.M. KELLER

Jos $E 40690$

Date $4-2-97$

Date $4-14-97$

$$
\begin{aligned}
& P_{v}=.0613 \frac{\mathrm{lbm}}{\mathrm{ft}^{3}}\left(\frac{1246 \mathrm{ft} / \mathrm{min}}{1097}\right)^{2}=.0791 \mathrm{in.} \mathrm{WG} . \\
& \Delta P=0.86(.0791)=.068 \mathrm{in.} \mathrm{WG} .
\end{aligned}
$$

$\Delta p$ is same for both sides of wye exhausted inlet.

Determine pressure losses for $250 \mathrm{cfm}$ in the remaining piping system (20 inlet riser, 12"outlet, 105 duct, 106 outlet, 106 duct)

$$
\begin{aligned}
& \Delta p=f\left(\frac{12 L}{D_{n}}\right) \rho\left(\frac{V}{1097}\right)^{2} \quad p 32.4 \text { en } 19 \\
& \Delta p=\text { pressure loss (in W.G.) } \\
& f=\text { friction factor (Moody diagram) } \\
& L=\text { duct length } \\
& D=\text { duct diameter } \\
& \rho=.061316 \mathrm{~m} / \mathrm{ft}^{3} \text { (from above) } \\
& V=\text { velocity (fp) } \\
& R_{e}=\text { Reynold's number }=\frac{V D}{\nu} \\
& \nu=\text { kinematic viscosity }=2.12 E-4 \\
& V=\frac{Q}{A}=\frac{250 \mathrm{cfm}}{(\pi / 4)(\mathrm{D} / 2)^{2}} \\
& =2865 \text { fem } \quad 4^{\prime \prime} \text { duct } \\
& =1246 \text { fp 6"duct } \\
& =318 \text { nom } 12^{\prime \prime} \text { duct }
\end{aligned}
$$

BD -6400-060.1 (07/93) 
HNF-SD-WM-DA-236, Rev 0

Page $C-21$

ANALYTICAL CALCULATIONS

Page 2 2 of 21 .

subject Call $M-3$

originator A.F. Weller

Checker C.M.KELLER

$$
R_{e}=\frac{V D}{\nu}=\frac{(2865)(4 / 12)}{(2.12 E-4)(60)}
$$

Roughness $\epsilon=.00015^{\prime}$ (steel duct)

$$
\begin{array}{rlrl}
E / D=\frac{.00015}{4 / 12} & =.0005 & 4^{\prime \prime} \text { duct } \\
& =.0003 \quad 6^{\prime \prime} \text { duct } \\
& =.00015 \quad 12^{\prime \prime} \text { duct }
\end{array}
$$

Friction factors (Moody diagram)

$$
\begin{aligned}
f & =0.021 & 4^{\prime \prime} \text { duct } \\
& =0.022 & \quad 6^{\prime \prime} \text { duct } \\
& =0.024 & 12^{\prime \prime} \text { duct }
\end{aligned}
$$

Calculate $\Delta p$ for $100^{\prime}$ of duct with $250 \mathrm{cfm}$

$$
\begin{aligned}
\Delta p=f\left(\frac{12 L}{D}\right) & e\left(\frac{V}{1097}\right)^{2} \\
4^{\prime \prime} \text { duct } \Delta p & =.021\left(\frac{12(100)}{4}\right)(.0613)\left(\frac{2865}{1097}\right)^{2} \\
\Delta p_{4} & =2.6 \text { in W.G. } / 100^{\prime} \\
\Delta p_{6} & =0.35 \text { in W.G. } / 100^{\prime} \\
\Delta p_{12} & =0.01 \text { in W.G. } / 100^{\prime}
\end{aligned}
$$

BD -6400-060.1 (07/93) 
HNF-SD-WM-DA-236, Rev 0

Page $\mathrm{C}-22$

ANALYTICAL CALCULATIONS

Page $2 \%$ of 21

subject Cate $M-3$ 40690

Originator A.E. Weller Date $4-2-97$ Checker C.M. KELLER Date $4-14-97$

Calculate $\Delta p$ for each duct run from tank inlet. to exhausted inlet.

for $B ! /-105$

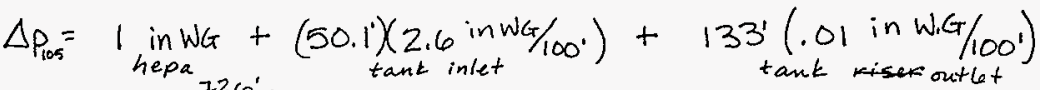

$$
\begin{aligned}
& +\begin{array}{r}
72.0^{\prime} \\
+T_{\text {duct }}^{\prime}\left(0.35 \text { in W.G. } / 100^{\prime}\right)+ \\
\text { exhausted inc. }
\end{array} \\
& \text { exhausted inlet } \\
& \Delta P_{105}=2.63 \text { in. W.G. }
\end{aligned}
$$

for $3 y-106$

$$
\begin{aligned}
\Delta P_{106}= & 1 \text { in WG }+\left(50.1^{\circ}\right)\left(2.6^{\text {inWG/100 }}\right)+\left(65.7^{\prime}\right)\left(0.35^{\mathrm{inWG/100}}\right) \\
& +\left(198.6^{\circ}\right)\left(0.35^{\circ} \mathrm{inW/100^{ \circ }}\right)+.068 \text { in WG } \\
\Delta P_{106}= & 3.29 \text { in W.G. }
\end{aligned}
$$

Note: Most of the losses are due to the tank inlet loss, which is 2.3 in.W.G.

* A difference of 0.7 in W.G. exists in the two duct runs. This is a small amount and should not cause any problems in balancing the whole system.

BD-6400-060.1 (07/93) 


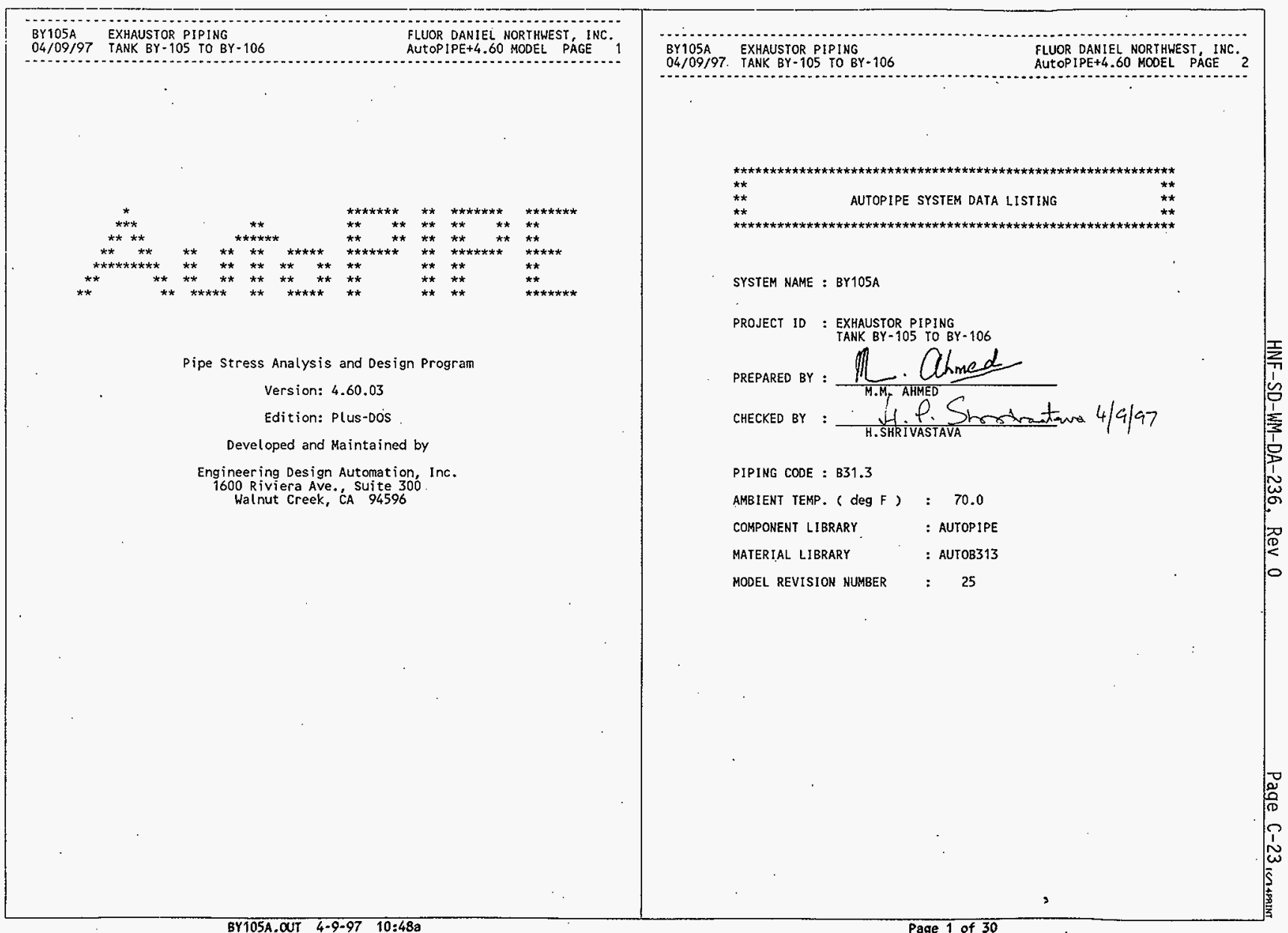




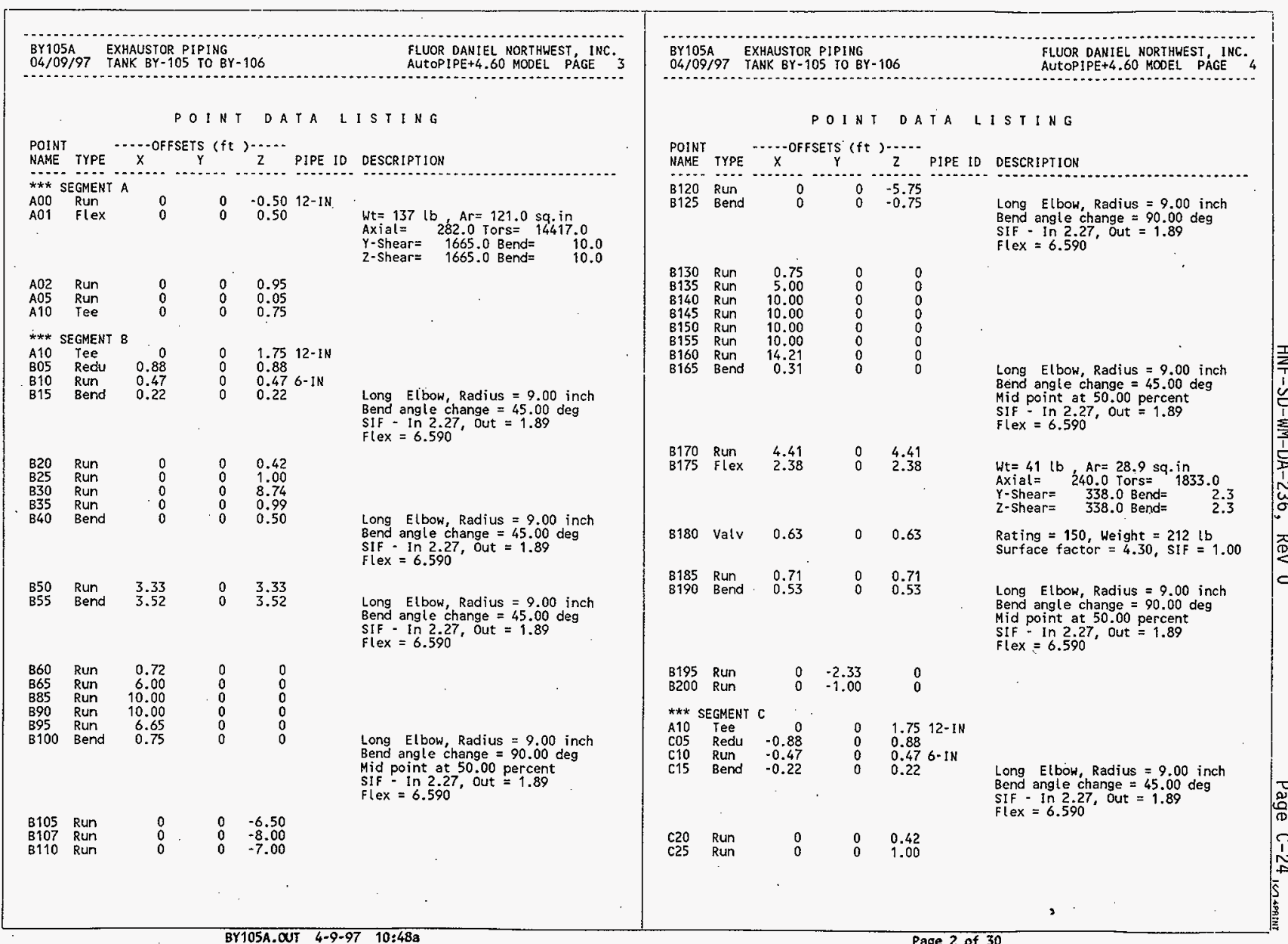




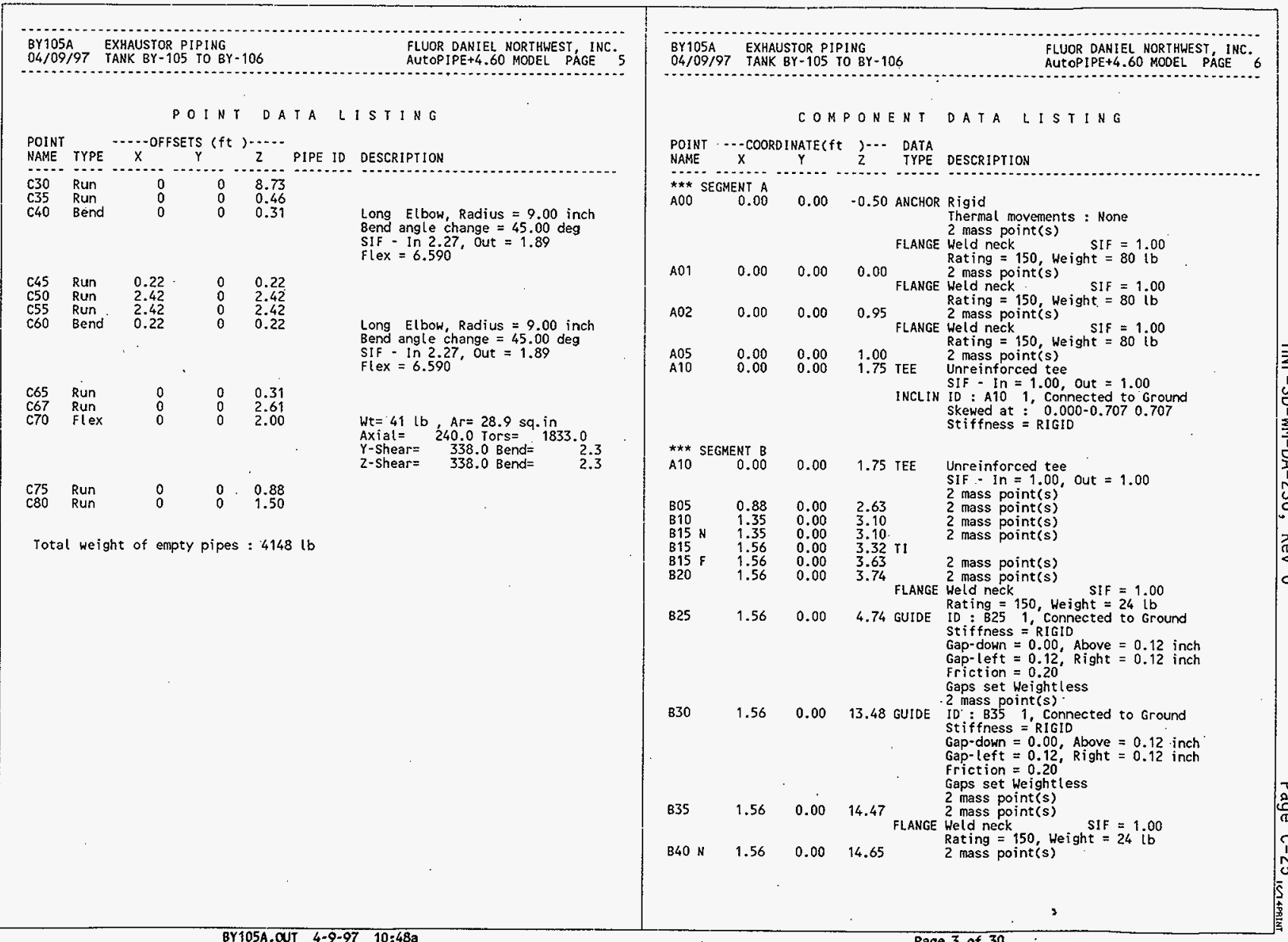




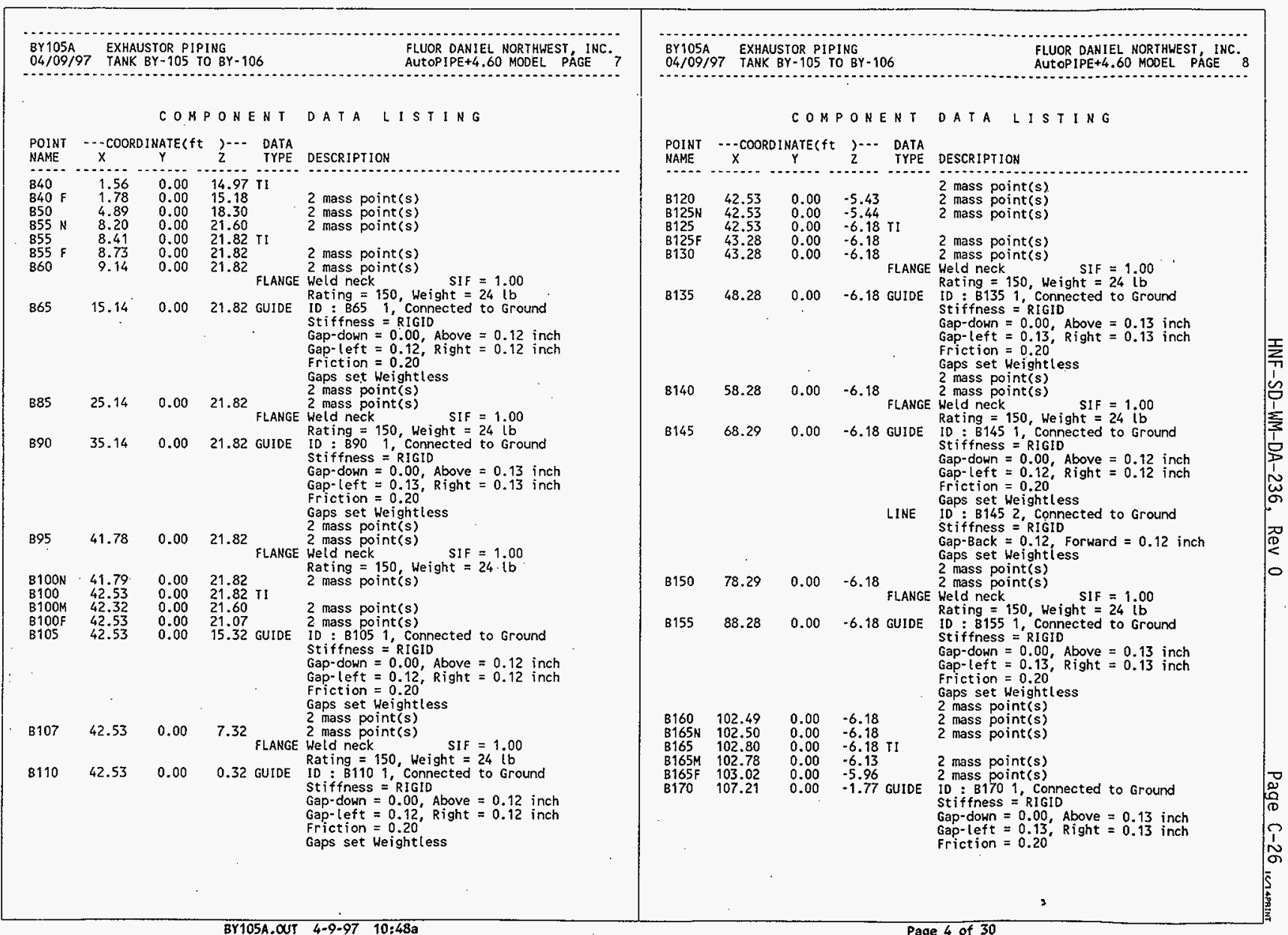




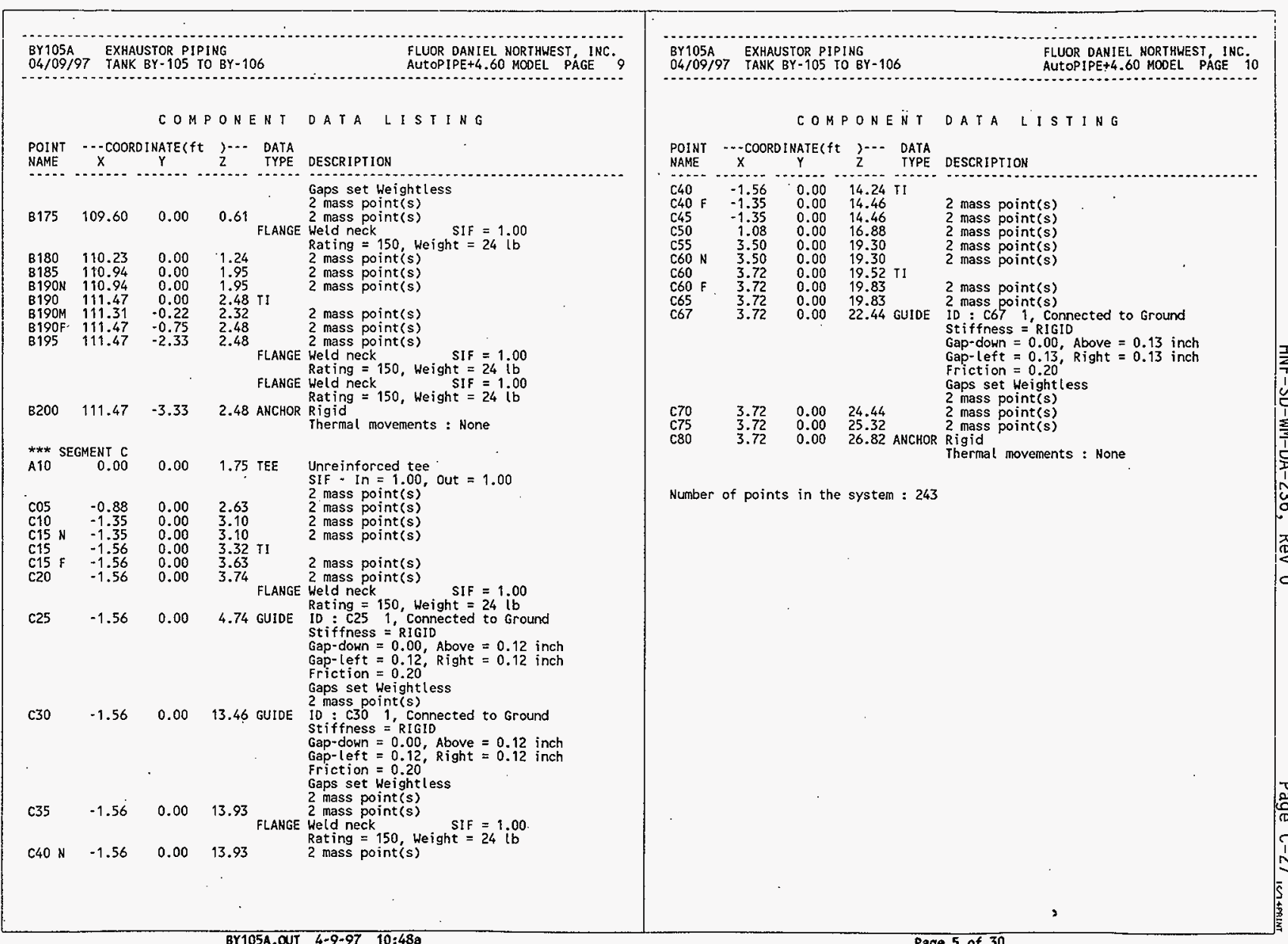




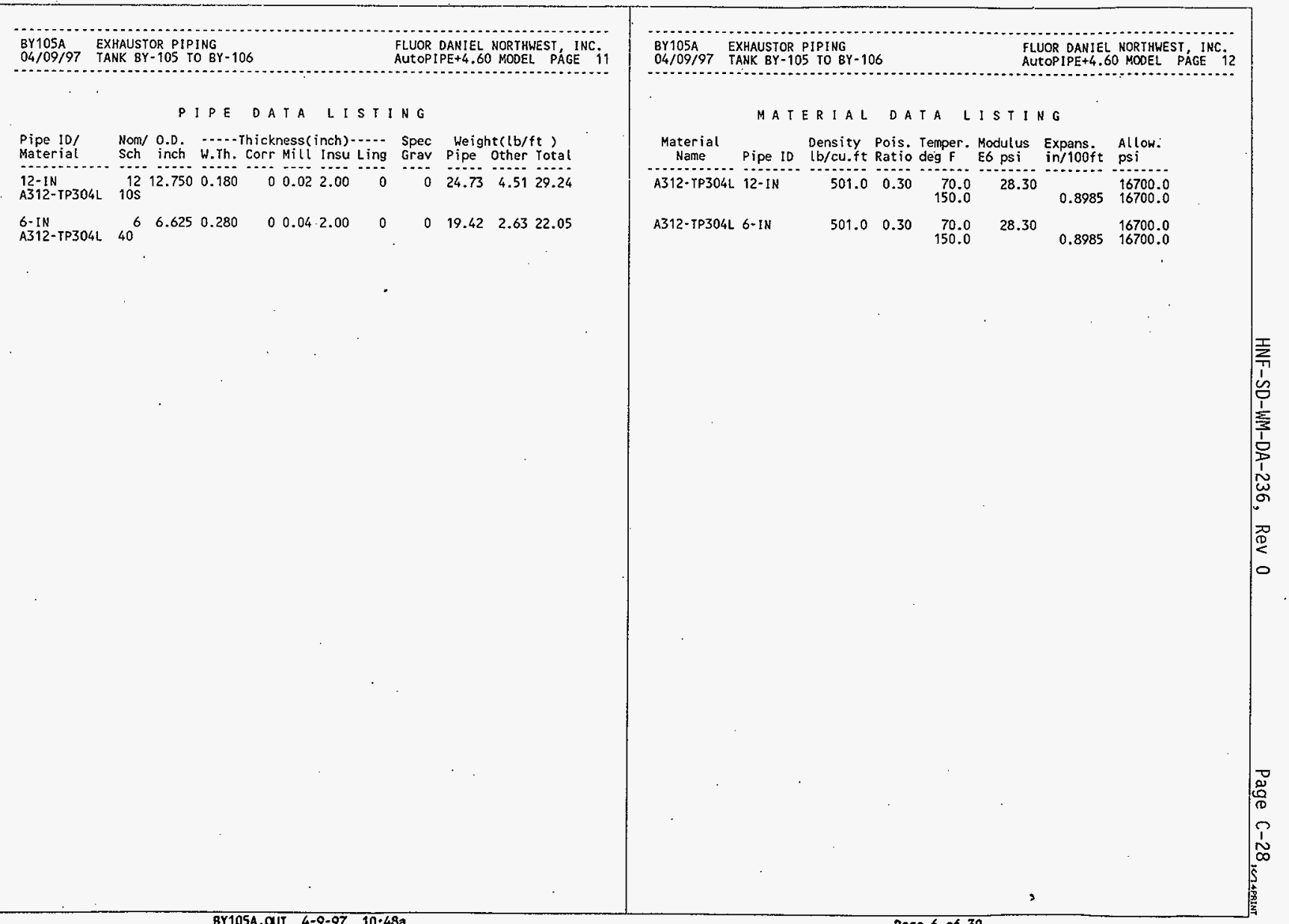




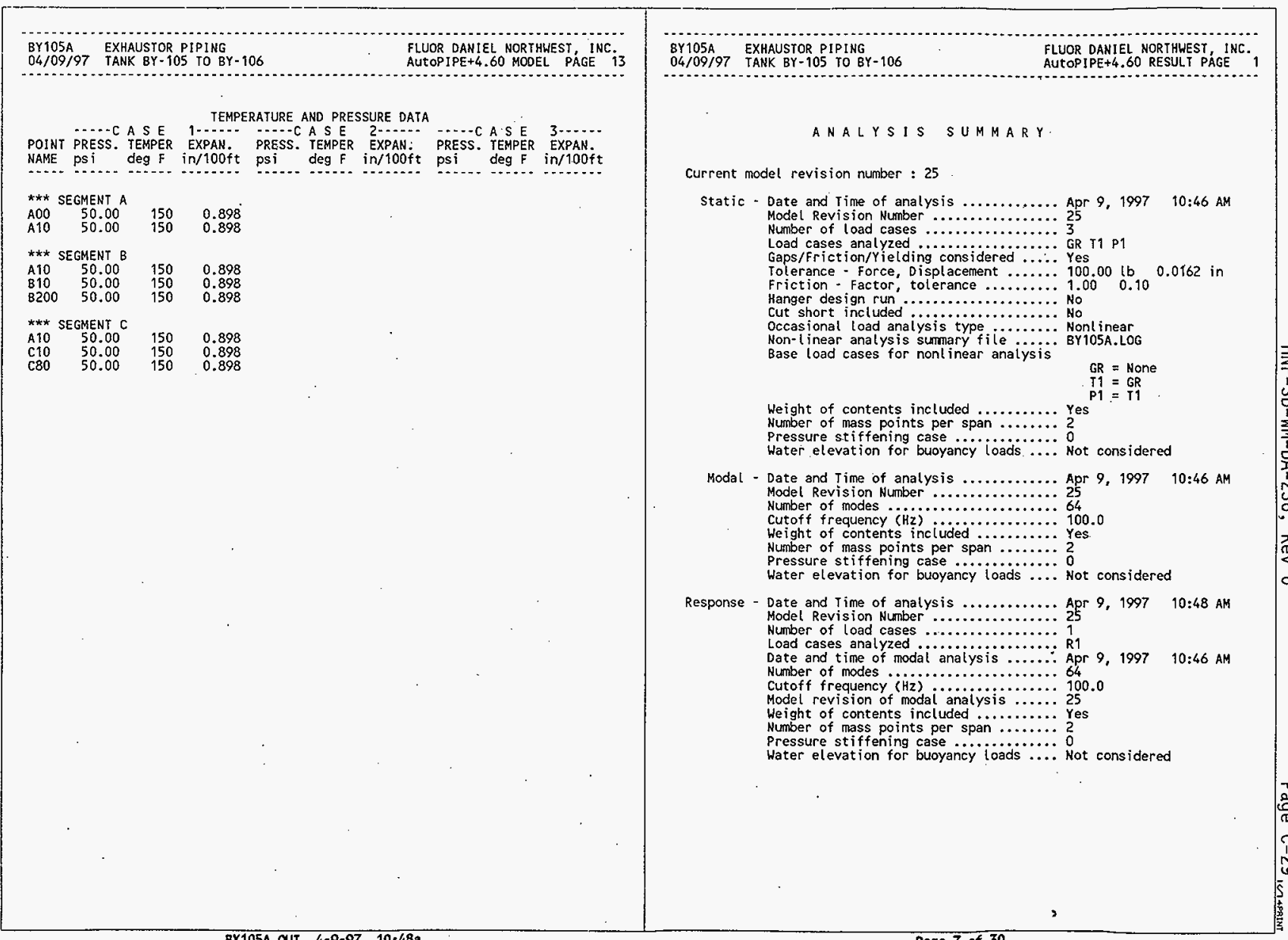




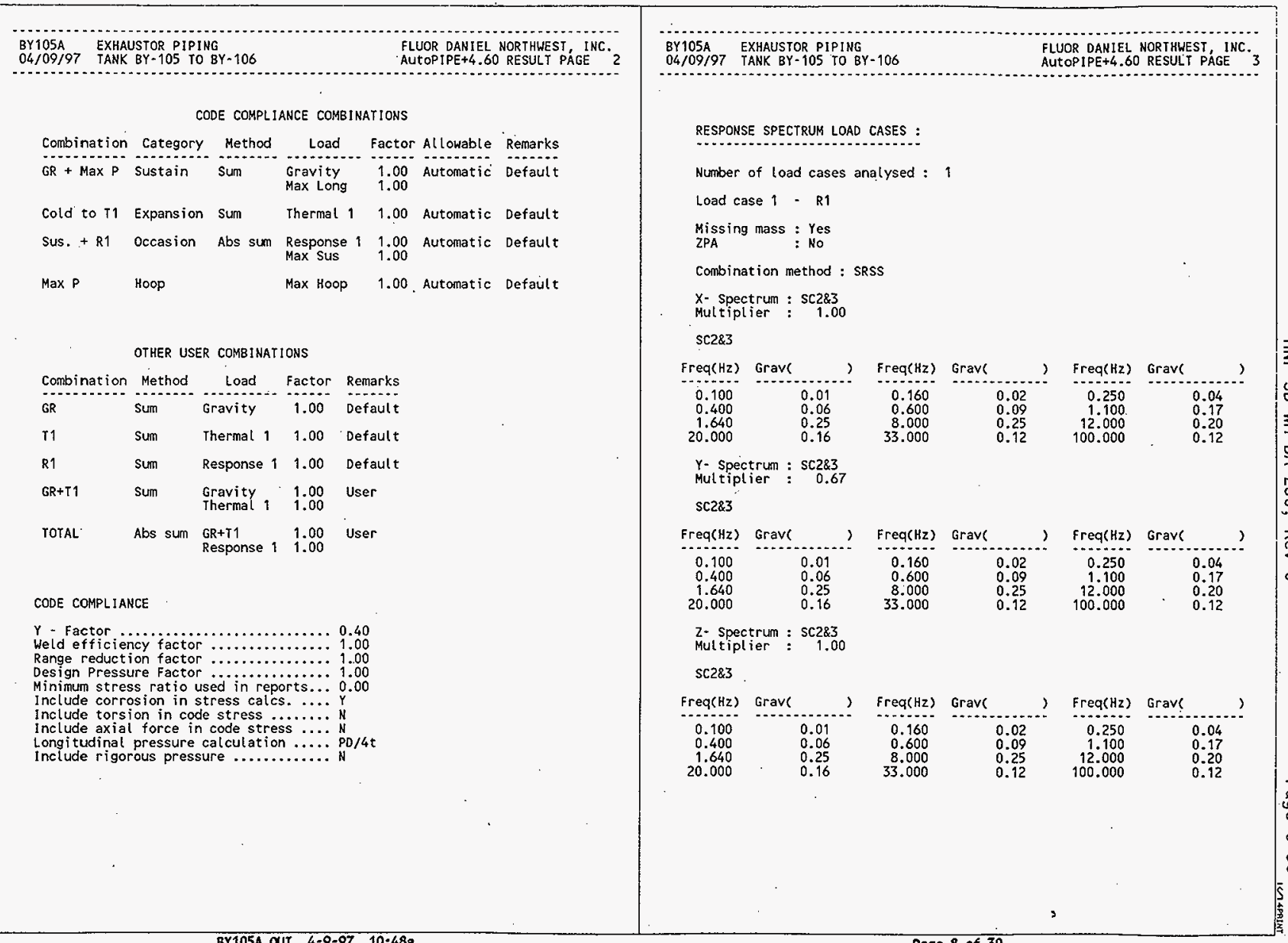




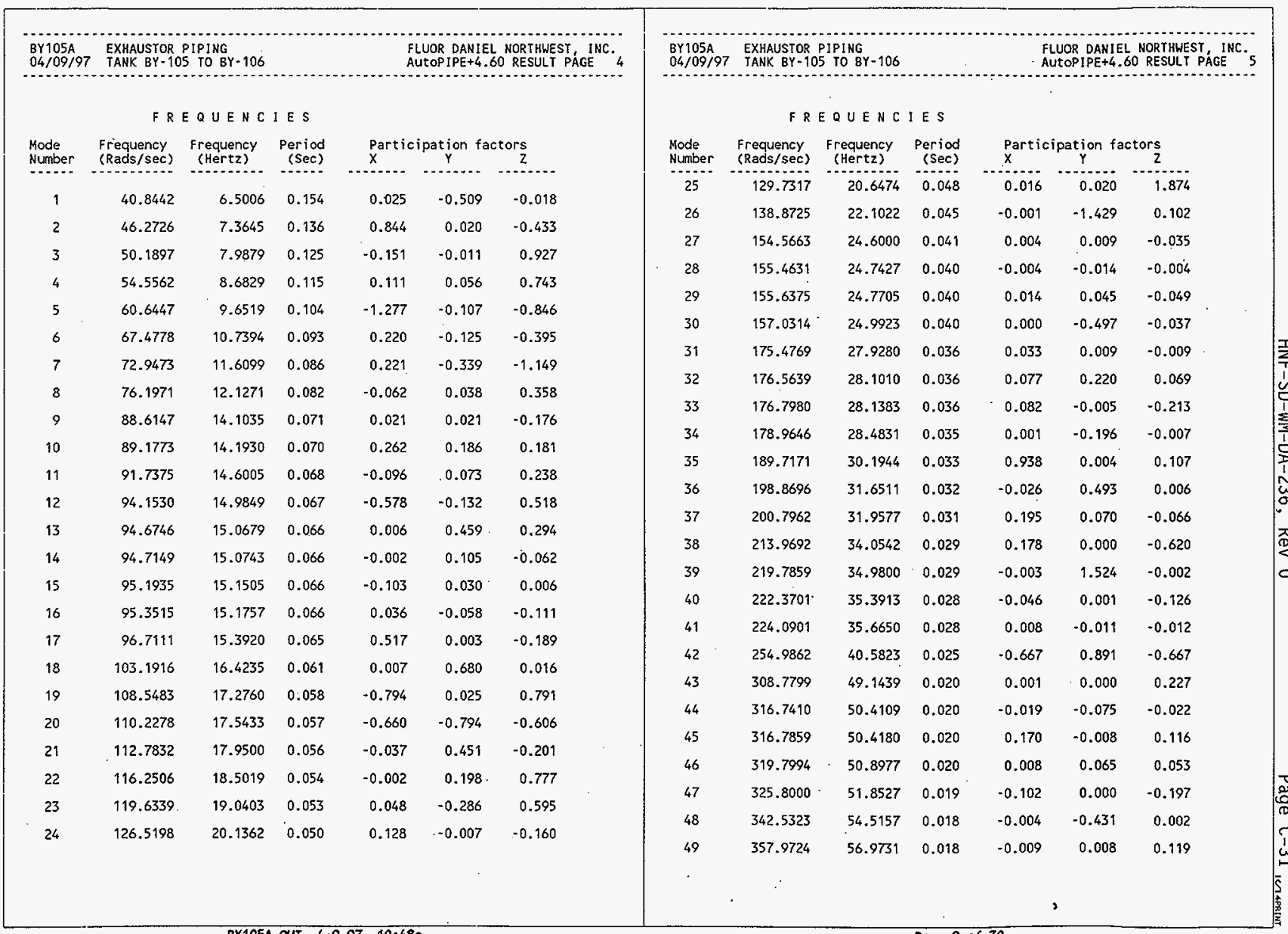




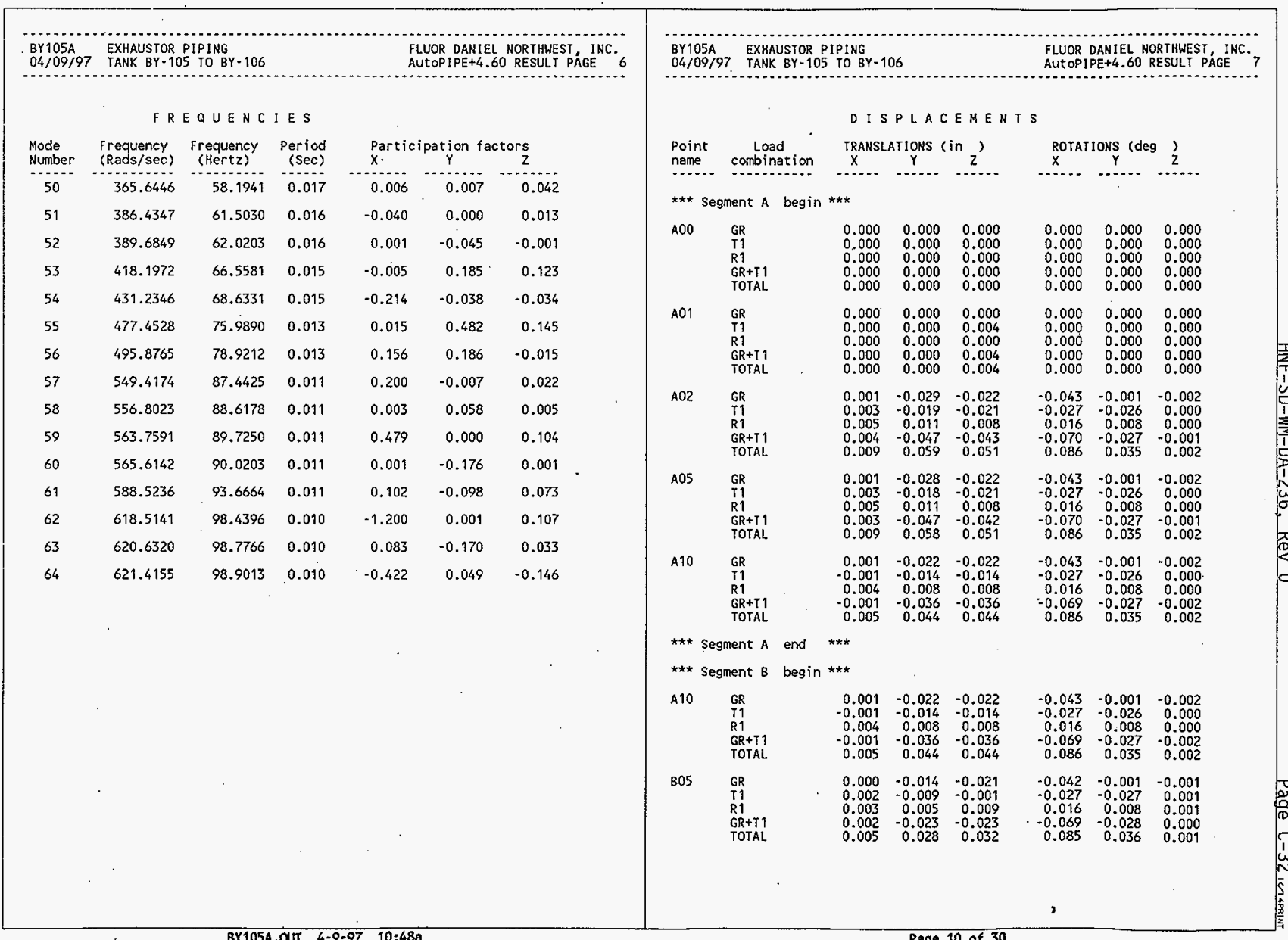




\begin{tabular}{|c|c|c|c|c|c|c|c|c|c|c|c|c|c|c|c|}
\hline \multicolumn{5}{|c|}{$\begin{array}{ll}\text { BY } 105 A & \text { EXHAUSTOR PIPING } \\
04 / 09 / 97 & \text { TANK BY-105 TO BY-106 }\end{array}$} & \multicolumn{3}{|c|}{$\begin{array}{l}\text { FLUOR DANIEL NORTHWEST, INC. } \\
\text { AUTOPIPE+4.60 RESULT PAGE } 8\end{array}$} & \multicolumn{5}{|c|}{$\begin{array}{ll}\text { BY105A } & \text { EXHAUSYOR PIPING } \\
04 / 09 / 97 & \text { TANK BY-105 to } 8 Y-106\end{array}$} & \multicolumn{3}{|c|}{$\begin{array}{l}\text { FLUOR DAN1EL NORTHHEST, IHC. } \\
\text { AutOPIPE+4.60 RESULT PAGE }\end{array}$} \\
\hline \multicolumn{8}{|c|}{ DISPLACEMENTS } & \multicolumn{8}{|c|}{ DISPLACEMENTS } \\
\hline $\begin{array}{l}\text { Point } \\
\text { name }\end{array}$ & $\begin{array}{l}\text { Load } \\
\text { combination }\end{array}$ & $\begin{array}{l}\text { TRANSLA } \\
\times\end{array}$ & ATIONS & in $z^{\prime}$ & $\begin{array}{l}\text { ROTAT } \\
x\end{array}$ & IONS c des & $9 \frac{1}{2}$ & $\begin{array}{l}\text { Point } \\
\text { name }\end{array}$ & $\begin{array}{l}\text { Load } \\
\text { combination }\end{array}$ & TRANSLA & $\underset{Y}{A T I O N S C}$ & (in ${ }_{2}^{\prime}$ & $\begin{array}{l}\text { ROIAT } \\
x\end{array}$ & I IONS (deg & 23 \\
\hline B10 & $\begin{array}{l}\text { GR } \\
T 1 \\
R 1 \\
\text { GR+T1 } \\
\text { TOTAL }\end{array}$ & $\begin{array}{l}0.000 \\
0.003 \\
0.002 \\
0.004 \\
0.005\end{array}$ & $\begin{array}{r}-0.010 \\
-0.006 \\
0.004 \\
-0.016 \\
0.020\end{array}$ & $\begin{array}{r}-0.021 \\
0.006 \\
0.009 \\
-0.016 \\
0.025\end{array}$ & $\begin{array}{r}-0.041 \\
-0.026 \\
0.016 \\
-0.068 \\
0.083\end{array}$ & $\begin{array}{r}-0.001 \\
-0.028 \\
0.008 \\
-0.029 \\
0.036\end{array}$ & $\begin{array}{r}-0.001 \\
0.001 \\
0.001 \\
0.000 \\
0.001\end{array}$ & $B 40 \mathrm{~F}$ & $\begin{array}{l}\text { GR } \\
\text { T1 } \\
\text { R1 } \\
\text { GR+T1 } \\
\text { TOTAL }\end{array}$ & $\begin{array}{r}-0.002 \\
-0.134 \\
0.004 \\
-0.137 \\
0.141\end{array}$ & $\begin{array}{r}-0.008 \\
-0.002 \\
0.002 \\
-0.010 \\
0.019\end{array}$ & $\begin{array}{r}-0.021 \\
0.117 \\
0.009 \\
0.096 \\
0.105\end{array}$ & $\begin{array}{l}0.027 \\
0.004 \\
0.005 \\
0.031 \\
0.036\end{array}$ & $\begin{array}{r}-0.001 \\
-0.032 \\
0.016 \\
-0.033 \\
0.049\end{array}$ & $\begin{array}{r}-0.004 \\
0.002 \\
0.002 \\
-0.002 \\
0.003\end{array}$ \\
\hline B15 N & $\begin{array}{l}\text { GR } \\
T 1 \\
R 1 \\
\text { GR+T1. } \\
\text { TOTAL }\end{array}$ & $\begin{array}{l}0.000 \\
0.003 \\
0.002 \\
0.004 \\
0.005\end{array}$ & $\begin{array}{r}-0.010 \\
-0.006 \\
0.004 \\
-0.016 \\
0.020\end{array}$ & $\begin{array}{r}-0.021 \\
0.006 \\
0.009 \\
.0 .016 \\
0.025\end{array}$ & $\begin{array}{r}-0.041 \\
-0.026 \\
0.016 \\
-0.068 \\
0.083\end{array}$ & $\begin{array}{r}-0.001 \\
-0.028 \\
0.008 \\
-0.029 \\
0.036\end{array}$ & $\begin{array}{r}-0.001 \\
0.001 \\
0.001 \\
0.000 \\
0.001\end{array}$ & $B 50$ & $\begin{array}{l}G R \\
T 1 \\
R 1 \\
\text { GR+T1 } \\
\text { TOTAL }\end{array}$ & $\begin{array}{r}-0.003 \\
-0.120 \\
0.015 \\
-0.123 \\
0.138\end{array}$ & $\begin{array}{r}-0.026 \\
-0.003 \\
0.005 \\
-0.029 \\
0.034\end{array}$ & $\begin{array}{r}-0.021 \\
0.159 \\
0.009 \\
0.138 \\
0.147\end{array}$ & $\begin{array}{l}0.027 \\
0.003 \\
0.006 \\
0.031 \\
0.037\end{array}$ & $\begin{array}{r}-0.001 \\
+0.010 \\
0.015 \\
-0.011 \\
0.026\end{array}$ & $\begin{array}{l}0.006 \\
0.003 \\
0.002 \\
0.009 \\
0.010\end{array}$ \\
\hline B15 F & $\begin{array}{l}\text { GR } \\
T 1 \\
\text { R1 } \\
\text { GR+T1 } \\
\text { TOTAL }\end{array}$ & $\begin{array}{l}0.000 \\
0.001 \\
0.001 \\
0.001 \\
0.002\end{array}$ & $\begin{array}{r}-0.006 \\
-0.004 \\
0.002 \\
-0.010 \\
0.012\end{array}$ & $\begin{array}{r}-0.021 \\
0.012 \\
0.009 \\
-0.010 \\
0.019\end{array}$ & $\begin{array}{r}-0.029 \\
-0.018 \\
0.011 \\
-0.046 \\
.0 .057\end{array}$ & $\begin{array}{r}-0.001 \\
-0.046 \\
0.005 \\
-0.047 \\
0.052\end{array}$ & $\begin{array}{r}-0.004 \\
-0.001 \\
0.001 \\
-0.005 \\
0.006\end{array}$ & B55 N & $\begin{array}{l}G R \\
T 1 \\
R 1 \\
G R+T 1 \\
\text { TOTAL }\end{array}$ & $\begin{array}{r}-0.004 \\
-0.090 \\
0.022 \\
-0.093 \\
0.115\end{array}$ & $\begin{array}{r}-0.034 \\
-0.003 \\
0.008 \\
-0.037 \\
0.045\end{array}$ & $\begin{array}{l}-0.020 \\
0.188 \\
0.014 \\
0.168 \\
0.182\end{array}$ & $\begin{array}{l}0.024 \\
0.003 \\
0.007 \\
0.027 \\
0.033\end{array}$ & $\begin{array}{r}.0 .001 \\
0.013 \\
0.006 \\
0.012 \\
0.018\end{array}$ & $\begin{array}{l}0.021 \\
0.003 \\
0.004 \\
0.023 \\
0.027\end{array}$ \\
\hline B20 & $\begin{array}{l}\text { GR } \\
T 1 \\
R 1 \\
\text { GR+T1 } \\
\text { TOTAL }\end{array}$ & $\begin{array}{l}0.000 \\
0.000 \\
0.001 \\
0.000 \\
0.001\end{array}$ & $\begin{array}{r}-0.005 \\
-0.003 \\
0.002 \\
-0.009 \\
0.011\end{array}$ & $\begin{array}{r}-0.021 \\
0.013 \\
0.009 \\
-0.009 \\
0.018\end{array}$ & $\begin{array}{r}-0.028 \\
-0.017 \\
0.010 \\
-0.046 \\
0.056\end{array}$ & $\begin{array}{r}-0.001 \\
-0.046 \\
0.005 \\
-0.048 \\
0.053\end{array}$ & $\begin{array}{r}-0.004 \\
-0.001 \\
0.001 \\
-0.005 \\
0.006\end{array}$ & B55 F & $\begin{array}{l}\text { GR } \\
\text { T1 } \\
R 1 \\
\text { GR+T1 } \\
\text { TOTAL }\end{array}$ & $\begin{array}{r}-0.004 \\
-0.084 \\
0.022 \\
-0.088 \\
0.110\end{array}$ & $\begin{array}{r}-0.032 \\
-0.003 \\
0.008 \\
-0.035 \\
0.043\end{array}$ & $\begin{array}{c}-0.020 \\
0.187 \\
0.014 \\
0.167 \\
0.181\end{array}$ & $\begin{array}{l}0.021 \\
0.003 \\
0.006 \\
0.024 \\
0.030\end{array}$ & $\begin{array}{r}-0.001 \\
0.031 \\
0.006 \\
0.030 \\
0.037\end{array}$ & $\begin{array}{l}0.028 \\
0.003 \\
0.005 \\
0.030 \\
0.036\end{array}$ \\
\hline B25 & $\begin{array}{l}\text { GR } \\
T 1 \\
\text { R1 } \\
\text { GR+I1 } \\
\text { JOTAL }\end{array}$ & $\begin{array}{r}0.000 \\
-0.010 \\
0.000 \\
-0.010 \\
0.010\end{array}$ & $\begin{array}{l}0.000 \\
0.000 \\
0.000 \\
0.000 \\
0.000\end{array}$ & $\begin{array}{r}-0.021 \\
0.022 \\
0.009 \\
0.000 \\
0.010\end{array}$ & $\begin{array}{r}-0.022 \\
-0.013 \\
0.008 \\
-0.035 \\
0.044\end{array}$ & $\begin{array}{r}-0.001 \\
-0.051 \\
0.005 \\
-0.052 \\
0.057\end{array}$ & $\begin{array}{r}-0.004 \\
-0.001 \\
0.001 \\
-0.005 \\
0.005\end{array}$ & 860 & $\begin{array}{l}\text { GR } \\
\text { I1 } \\
R 1 \\
\text { GR+T1 } \\
\text { TOTAL }\end{array}$ & $\begin{array}{r}-0.004 \\
-0.080 \\
0.022 \\
-0.084 \\
0.106\end{array}$ & $\begin{array}{r}-0.030 \\
-0.003 \\
0.008 \\
-0.033 \\
0.040\end{array}$ & $\begin{array}{l}-0.020 \\
0.184 \\
0.014 \\
0.164 \\
0.178\end{array}$ & $\begin{array}{l}0.021 \\
0.003 \\
0.006 \\
0.024 \\
0.030\end{array}$ & $\begin{array}{c}-0.001 \\
0.033 \\
0.007 \\
0.032 \\
0.039\end{array}$ & $\begin{array}{l}0.029 \\
0.003 \\
0.005 \\
0.031 \\
0.036\end{array}$ \\
\hline$B 30$ & $\begin{array}{l}\text { GR } \\
\text { T1 } \\
\text { R1 } \\
\text { GR+I1 } \\
\text { TOTAL }\end{array}$ & $\begin{array}{r}-0.002 \\
-0.118 \\
0.000 \\
-0.120 \\
0.120\end{array}$ & $\begin{array}{l}0.000 \\
0.000 \\
0.000 \\
0.000 \\
0.000\end{array}$ & $\begin{array}{r}-0.021 \\
0.100 \\
0.009 \\
0.079 \\
0.088\end{array}$ & $\begin{array}{l}0.017 \\
0.006 \\
0.004 \\
0.023 \\
0.027\end{array}$ & $\begin{array}{r}-0.001 \\
-0.056 \\
0.008 \\
-0.057 \\
0.066\end{array}$ & $\begin{array}{r}-0.003 \\
0.001 \\
0.001 \\
-0.001 \\
0.002\end{array}$ & B65 & $\begin{array}{l}\text { GR } \\
\text { T1 } \\
R 1 \\
\text { GR+T1 } \\
\text { TOTAL }\end{array}$ & $\begin{array}{r}-0.004 \\
-0.026 \\
0.022 \\
-0.030 \\
0.052\end{array}$ & $\begin{array}{l}0.000 \\
0.000 \\
0.000 \\
0.000 \\
0.000\end{array}$ & $\begin{array}{r}-0.019 \\
0.139 \\
0.000 \\
0.120 \\
0.120\end{array}$ & $\begin{array}{l}0.019 \\
0.002 \\
0.006 \\
0.022 \\
0.028\end{array}$ & $\begin{array}{r}-0.001 \\
0.030 \\
0.011 \\
0.030 \\
0.041\end{array}$ & $\begin{array}{l}0.007 \\
0.002 \\
0.006 \\
0.008 \\
0.015\end{array}$ \\
\hline B35 & $\begin{array}{l}\text { GR } \\
\text { T1 } \\
\text { R1 } \\
\text { GR+T1 } \\
\text { TOTAL }\end{array}$ & $\begin{array}{r}-0.002 \\
-0.129 \\
0.002 \\
-0.132 \\
0.134\end{array}$ & $\begin{array}{r}-0.004 \\
-0.001 \\
0.001 \\
-0.005 \\
0.006\end{array}$ & $\begin{array}{l}-0.021 \\
0.109 \\
0.009 \\
0.088 \\
0.097\end{array}$ & $\begin{array}{l}0.020 \\
0.006 \\
0.004 \\
0.026 \\
0.030\end{array}$ & $\begin{array}{r}-0.001 \\
-0.052 \\
0.011 \\
-0.053 \\
0.064\end{array}$ & $\begin{array}{r}-0.002 \\
0.002 \\
0.001 \\
-0.001 \\
0.002\end{array}$ & 885 & $\begin{array}{l}\text { GR } \\
\text { II } \\
\text { RI } \\
\text { GR+T1 } \\
\text { TOTAL }\end{array}$ & $\begin{array}{r}-0.004 \\
0.063 \\
0.022 \\
0.060 \\
0.082\end{array}$ & $\begin{array}{r}-0.021 \\
0.001 \\
0.009 \\
-0.020 \\
0.029\end{array}$ & $\begin{array}{c}-0.018 \\
0.117 \\
0.014 \\
0.099 \\
0.113\end{array}$ & $\begin{array}{l}0.017 \\
0.002 \\
0.006 \\
0.018 \\
0.025\end{array}$ & $\begin{array}{r}0.000 \\
-0.005 \\
0.003 \\
-0.005 \\
0.009\end{array}$ & $\begin{array}{r}-0.003 \\
0.000 \\
0.001 \\
-0.003 \\
0.004\end{array}$ \\
\hline $840 \mathrm{~N}$ & $\begin{array}{l}\text { GR } \\
\text { T1 } \\
\text { R1 } \\
\text { GR+r1 } \\
\text { TOTAL }\end{array}$ & $\begin{array}{r}-0.002 \\
-0.131 \\
0.002 \\
-0.134 \\
0.136 \\
.\end{array}$ & $\begin{array}{r}-0.005 \\
-0.001 \\
0.001 \\
-0.006 \\
0.007\end{array}$ & $\begin{array}{l}-0.021 \\
0.111 \\
0.009 \\
0.089 \\
0.099\end{array}$ & $\begin{array}{l}0.021 \\
0.005 \\
0.004 \\
0.026 \\
0.031\end{array}$ & $\begin{array}{r}-0.001 \\
-0.052 \\
0.011 \\
-0.053 \\
0.064\end{array}$ & $\begin{array}{r}-0.002 \\
0.002 \\
0.001 \\
-0.001 \\
0.002\end{array}$ & 890 & $\begin{array}{l}\text { GR } \\
\text { I1 } \\
\text { R1 } \\
\text { GR+T1 } \\
\text { TOTAL }\end{array}$ & $\begin{array}{r}-0.004 \\
0.153 \\
0.022 \\
0.149 \\
0.172\end{array}$ & $\begin{array}{l}0.000 \\
0.000 \\
0.000 \\
0.000 \\
0.000\end{array}$ & $\begin{array}{r}-0.017 \\
0.142 \\
0.000 \\
0.125 \\
0.125\end{array}$ & $\begin{array}{l}0.014 \\
0.001 \\
0.007 \\
0.015 \\
0.021\end{array}$ & $\begin{array}{r}0.000 \\
-0.015 \\
0.015 \\
-0.015 \\
0.030\end{array}$ & $\begin{array}{r}0.005 \\
-0.001 \\
0.006 \\
0.004 \\
0.010\end{array}$ \\
\hline
\end{tabular}




\begin{tabular}{|c|c|c|c|c|c|c|c|c|c|c|c|c|c|c|c|c|}
\hline \multicolumn{5}{|c|}{$\begin{array}{ll}\text { BY 105A } & \text { EXHAUSTOR PIPING } \\
04 / 09 / 97 & \text { TANK BY-105 TO BY-106 }\end{array}$} & \multicolumn{3}{|c|}{$\begin{array}{l}\text { FLUOR DANIEL NORTHWEST INC } \\
\text { AUTOPIPE+4.60 RESULT PÁGE io }\end{array}$} & \multicolumn{5}{|c|}{$\begin{array}{l}\text { BY105A } \\
04 / 09 / 97 \text { EXHAUSTOR P1PING } \\
\text { TANK BY } 105 \text { TO BY- } 105\end{array}$} & \multicolumn{3}{|c|}{$\begin{array}{l}\text { FLUOR DANIEL NORTHWEST, INC } \\
\text { AUt OPIPE+4.60 RESULT PAGE }\end{array}$} & \\
\hline \multicolumn{8}{|c|}{ DISPLACEMENTS } & \multicolumn{8}{|c|}{ DISPLACEMENTS } & \\
\hline $\begin{array}{l}\text { Point } \\
\text { name }\end{array}$ & $\begin{array}{l}\text { Load } \\
\text { combination }\end{array}$ & $\underset{X}{\text { TRANSLA }}$ & ATIONS & $\operatorname{lin}_{2}^{\prime}$ & $\begin{array}{l}\text { ROTAT } \\
x\end{array}$ & ONS cdeg & 92 & $\begin{array}{l}\text { Point } \\
\text { name }\end{array}$ & $\begin{array}{l}\text { Load } \\
\text { combination }\end{array}$ & $\underset{\mathrm{X}}{\mathrm{TRANSLA}}$ & $\underset{Y}{\text { ATIONS }} \underset{Y}{ }(i$ & $\left(\right.$ in $z^{\prime}$ & ROTAT & Ions (deg & ? & \\
\hline 895 & $\begin{array}{l}\text { GR } \\
\text { T1 } \\
R 1 \\
\text { GR+T1 } \\
\text { TOIAL }\end{array}$ & $\begin{array}{r}-0.004 \\
0.213 \\
0.022 \\
0.209 \\
0.231\end{array}$ & $\begin{array}{r}-0.010 \\
-0.001 \\
0.008 \\
-0.010 \\
0.019\end{array}$ & $\begin{array}{r}-0.017 \\
0.152 \\
0.024 \\
0.155 \\
0.159\end{array}$ & $\begin{array}{l}0.012 \\
0.001 \\
0.007 \\
0.013 \\
0.019\end{array}$ & $\begin{array}{l}0.000 \\
0.008 \\
0.016 \\
0.008 \\
0.024\end{array}$ & $\begin{array}{r}-0.010 \\
0.000 \\
0.006 \\
-0.011 \\
0.016\end{array}$ & B125N & $\begin{array}{l}\text { GR } \\
\text { II } \\
\text { RI } \\
\text { GR+II } \\
\text { rOTAL }\end{array}$ & $\begin{array}{c}-0.005 \\
-0.209 \\
0.001 \\
-0.214 \\
0.216\end{array}$ & $\begin{array}{r}-0.001 \\
0.000 \\
0.006 \\
-0.001 \\
0.007\end{array}$ & $\begin{array}{r}-0.017 \\
-0.097 \\
0.026 \\
-0.114 \\
0.140\end{array}$ & $\begin{array}{r}-0.005 \\
0.000 \\
0.006 \\
-0.005 \\
0.011\end{array}$ & $\begin{array}{l}0.000 \\
0.067 \\
0.003 \\
0.067 \\
0.070\end{array}$ & $\begin{array}{l}0.008 \\
0.000 \\
0.005 \\
0.008 \\
0.013\end{array}$ & \\
\hline B100 N & $\begin{array}{l}G R \\
T 1 \\
R 1 \\
G R+T 1 \\
\text { TOTAL }\end{array}$ & $\begin{array}{r}-0.004 \\
0.213 \\
0.022 \\
0.209 \\
0.231\end{array}$ & $\begin{array}{r}-0.010 \\
-0.001 \\
0.008 \\
-0.010 \\
0.019\end{array}$ & $\begin{array}{r}-0.017 \\
0.152 \\
0.024 \\
0.135 \\
0.159\end{array}$ & $\begin{array}{l}0.012 \\
0.001 \\
0.007 \\
0.013 \\
0.019\end{array}$ & $\begin{array}{l}0.000 \\
0.008 \\
0.016 \\
0.008 \\
0.024\end{array}$ & $\begin{array}{r}-0.010 \\
0.000 \\
0.006 \\
-0.011 \\
0.016\end{array}$ & 8125F & $\begin{array}{l}\text { GR } \\
\text { I1 } \\
R 1 \\
\text { GR+I1 } \\
\text { JOTAL }\end{array}$ & $\begin{array}{r}-0.005 \\
-0.210 \\
0.000 \\
-0.215 \\
0.215\end{array}$ & $\begin{array}{r}-0.001 \\
0.000 \\
0.006 \\
-0.001 \\
0.008\end{array}$ & $\begin{array}{r}-0.017 \\
-0.109 \\
0.024 \\
-0.126 \\
0.149\end{array}$ & $\begin{array}{r}-0.013 \\
0.000 \\
0.006 \\
-0.013 \\
0.019 .\end{array}$ & $\begin{array}{l}0.000 \\
0.013 \\
0.021 \\
0.013 \\
0.034\end{array}$ & $\begin{array}{l}0.006 \\
0.000 \\
0.006 \\
0.006 \\
0.011\end{array}$ & \\
\hline B100M & $\begin{array}{l}\text { GR } \\
T 1 \\
R 1 \\
\text { GR+I1 } \\
\text { TOTAL }\end{array}$ & $\begin{array}{l}-0.004 \\
0.216 \\
0.022 \\
0.213 \\
0.235\end{array}$ & $\begin{array}{r}-0.010 \\
-0.001 \\
0.009 \\
-0.011 \\
0.020\end{array}$ & $\begin{array}{r}-0.017 \\
0.148 \\
0.025 \\
0.131 \\
0.156\end{array}$ & $\begin{array}{l}0.012 \\
0.001 \\
0.007 \\
0.013 \\
0.020\end{array}$ & $\begin{array}{l}0.000 \\
0.035 \\
0.014 \\
0.035 \\
0.050\end{array}$ & $\begin{array}{r}-0.009 \\
0.000 \\
0.005 \\
-0.009 \\
0.014\end{array}$ & B130 & $\begin{array}{l}\text { GR } \\
\text { I1 } \\
R 1 \\
\text { GR+I1 } \\
\text { TOTAL }\end{array}$ & $\begin{array}{r}-0.005 \\
-0.210 \\
0.000 \\
-0.215 \\
0.215\end{array}$ & $\begin{array}{r}-0.001 \\
0.000 \\
0.006 \\
-0.001 \\
0.008\end{array}$ & $\begin{array}{r}-0.017 \\
-0.109 \\
0.023 \\
-0.126 \\
0.149\end{array}$ & $\begin{array}{r}-0.0 .13 \\
0.000 \\
0.006 \\
-0.013 \\
0.019\end{array}$ & $\begin{array}{l}0.000 \\
0.013 \\
0.021 \\
0.013 \\
0.034\end{array}$ & $\begin{array}{l}0.006 \\
0.000 \\
0.006 \\
0.006 \\
0.011\end{array}$ & $\begin{array}{l}\Gamma \\
1 \\
1 \\
1 \\
0 \\
1\end{array}$ \\
\hline $\mathrm{B} 100 \mathrm{~F}$ & $\begin{array}{l}\text { GR } \\
T 1 \\
R 1 \\
\text { GR+T1 } \\
\text { rOTAL }\end{array}$ & $\begin{array}{l}-0.004 \\
0.213 \\
0.020 \\
0.209 \\
0.229\end{array}$ & $\begin{array}{r}-0.009 \\
-0.001 \\
0.008 \\
-0.010 \\
0.018\end{array}$ & $\begin{array}{r}-0.017 \\
0.141 \\
0.026 \\
0.124 \\
0.150\end{array}$ & $\begin{array}{l}0.011 \\
0.001 \\
0.007 \\
0.012 \\
0.019\end{array}$ & $\begin{array}{l}0.000 \\
0.062 \\
0.017 \\
0.062 \\
0.079\end{array}$ & $\begin{array}{r}-0.008 \\
0.000 \\
0.005 \\
-0.009 \\
0.013\end{array}$ & B135 & $\begin{array}{l}\text { GR } \\
T 1 \\
R 1 \\
\text { GR+T1 } \\
\text { TOIAL }\end{array}$ & $\begin{array}{r}-0.005 \\
-0.165 \\
0.000 \\
-0.0170 \\
0.170\end{array}$ & $\begin{array}{l}0.000 \\
0.000 \\
0.000 \\
0.000 \\
0.000\end{array}$ & $\begin{array}{r}-0.017 \\
-0.108 \\
0.000 \\
-0.125 \\
0.125\end{array}$ & $\begin{array}{r}-0.026 \\
0.0000 \\
0.007 \\
-0.026 \\
0.033\end{array}$ & $\begin{array}{r}0.000 \\
-0.010 \\
0.020 \\
-0.010 \\
0.030\end{array}$ & $\begin{array}{r}-0.010 \\
0.000 \\
0.006 \\
-0.010 \\
0.016\end{array}$ & $\begin{array}{l}1 \\
1 \\
0 \\
1 \\
1 \\
w \\
0\end{array}$ \\
\hline B105 & $\begin{array}{l}\text { GR } \\
T 1 \\
R 1 \\
\text { GR+T1 } \\
\text { TOTAL }\end{array}$ & $\begin{array}{r}-0.004 \\
0.124 \\
0.000 \\
0.120 \\
0.120\end{array}$ & $\begin{array}{l}0.000 \\
0.000 \\
0.000 \\
0.000 \\
0.000\end{array}$ & $\begin{array}{r}-0.017 \\
0.090 \\
0.026 \\
0.072 \\
0.098\end{array}$ & $\begin{array}{r}-0.001 \\
0.000 \\
0.006 \\
-0.001 \\
0.006\end{array}$ & $\begin{array}{l}0.000 \\
0.078 \\
0.013 \\
0.078 \\
0.091\end{array}$ & $\begin{array}{r}-0.005 \\
0.000 \\
0.003 \\
-0.005 \\
0.008\end{array}$ & B140 & $\begin{array}{l}\text { GR } \\
T 1 \\
R 1 \\
\text { GR+T1 } \\
\text { TOTAL }\end{array}$ & $\begin{array}{r}-0.005 \\
-0.075 \\
0.000 \\
-0.080 \\
0.081\end{array}$ & $\begin{array}{r}-0.032 \\
0.000 \\
0.008 \\
-0.032 \\
0.040\end{array}$ & $\begin{array}{r}-0.017 \\
-0.073 \\
0.019 \\
-0.090 \\
0.109\end{array}$ & $\begin{array}{r}-0.052 \\
0.000 \\
0.011 \\
-0.052 \\
0.063\end{array}$ & $\begin{array}{r}0.000 \\
-0.020 \\
0.003 \\
-0.020 \\
0.023\end{array}$ & $\begin{array}{l}0.000 \\
0.000 \\
0.001 \\
0.000 \\
0.001\end{array}$ & $\begin{array}{l}\mathbb{R}_{0}^{\infty} \\
0 \\
0\end{array}$ \\
\hline B107 & $\begin{array}{l}\text { GR } \\
\text { Y } \\
R 1 \\
\text { GR+T1 } \\
\text { TOTAL }\end{array}$ & $\begin{array}{r}-0.004 \\
-0.002 \\
0.007 \\
-0.006 \\
0.013\end{array}$ & $\begin{array}{r}-0.010 \\
0.000 \\
0.004 \\
-0.010 \\
0.014\end{array}$ & $\begin{array}{r}-0.017 \\
0.018 \\
0.026 \\
0.001 \\
0.027\end{array}$ & $\begin{array}{l}0.000 \\
0.000 \\
0.001 \\
0.000 \\
0.001\end{array}$ & $\begin{array}{l}0.000 \\
0.075 \\
0.003 \\
0.075 \\
0.078\end{array}$ & $\begin{array}{l}0.000 \\
0.000 \\
0.002 \\
0.000 \\
0.003\end{array}$ & B145 & $\begin{array}{l}\text { GR } \\
\text { I1 } \\
R 1 \\
\text { GR+T1 } \\
\text { TOTAL }\end{array}$ & $\begin{array}{r}-0.005 \\
0.015 \\
0.000 \\
0.009 \\
0.009\end{array}$ & $\begin{array}{l}0.000 \\
0.000 \\
0.000 \\
0.000 \\
0.000\end{array}$ & $\begin{array}{r}-0.017 \\
-0.037 \\
0.000 \\
-0.053 \\
0.053\end{array}$ & $\begin{array}{r}-0.078 \\
-0.001 \\
0.016 \\
-0.078 \\
0.094\end{array}$ & $\begin{array}{r}0.000 \\
-0.012 \\
0.010 \\
-0.012 \\
0.022\end{array}$ & $\begin{array}{l}0.009 \\
0.000 \\
0.005 \\
0.009 \\
0.014\end{array}$ & \\
\hline$B 110$ & $\begin{array}{l}\text { GR } \\
\text { T1 } \\
R 1 \\
\text { GR+T1 } \\
\text { TOTAL }\end{array}$ & $\begin{array}{r}-0.005 \\
-0.115 \\
0.000 \\
-0.120 \\
0.120\end{array}$ & $\begin{array}{l}0.000 \\
0.000 \\
0.000 \\
0.000 \\
0.000\end{array}$ & $\begin{array}{r}-0.017 \\
-0.045 \\
0.026 \\
-0.062 \\
0.088\end{array}$ & $\begin{array}{l}0.006 \\
0.000 \\
0.005 \\
0.006 \\
0.010\end{array}$ & $\begin{array}{l}0.000 \\
0.081 \\
0.004 \\
0.081 \\
0.085\end{array}$ & $\begin{array}{l}0.004 \\
0.000 \\
0.003 \\
0.004 \\
0.008\end{array}$ & B150 & $\begin{array}{l}\text { GR } \\
T 1 \\
R 1 \\
\text { GR+T1 } \\
\text { TOTAL }\end{array}$ & $\begin{array}{l}-0.005 \\
0.104 \\
0.000 \\
0.099 \\
0.099\end{array}$ & $\begin{array}{c}-0.005 \\
0.000 \\
0.008 \\
-0.005 \\
0.013\end{array}$ & $\begin{array}{r}-0.016 \\
-0.027 \\
0.015 \\
-0.043 \\
0.058\end{array}$ & $\begin{array}{r}-0.104 \\
-0.001 \\
0.021 \\
-0.104 \\
0.125\end{array}$ & $\begin{array}{l}0.000 \\
0.000 \\
0.003 \\
0.000 \\
0.003\end{array}$ & $\begin{array}{l}0.004 \\
0.000 \\
0.001 \\
0.004 \\
0.005\end{array}$ & \\
\hline B120 & $\begin{array}{l}\text { GR } \\
\text { T1 } \\
R 1 \\
\text { GR+T1 } \\
\text { TOTAL }\end{array}$ & $\begin{array}{r}-0.005 \\
-0.209 \\
0.001 \\
-0.214 \\
0.216\end{array}$ & $\begin{array}{r}-0.001 \\
0.000 \\
0.006 \\
-0.001 \\
0.007\end{array}$ & $\begin{array}{r}-0.017 \\
-0.097 \\
0.026 \\
-0.114 \\
0.140\end{array}$ & $\begin{array}{r}-0.005 \\
0.000 \\
0.006 \\
-0.005 \\
0.011\end{array}$ & $\begin{array}{l}0.000 \\
0.067 \\
0.003 \\
0.067 \\
0.070\end{array}$ & $\begin{array}{l}0.008 \\
0.000 \\
0.005 \\
0.008 \\
0.013\end{array}$ & B155 & $\begin{array}{l}\text { GR } \\
\text { T1 } \\
\text { R1 } \\
\text { GR+I1 } \\
\text { TOTAL }\end{array}$ & $\begin{array}{r}-0.005 \\
0.194 \\
0.000 \\
0.189 \\
0.189\end{array}$ & $\begin{array}{l}0.000 \\
0.000 \\
0.000 \\
0.000 \\
0.000\end{array}$ & $\begin{array}{r}-0.015 \\
-0.027 \\
0.000 \\
-0.043 \\
0.043\end{array}$ & $\begin{array}{r}-0.130 \\
-0.001 \\
0.026 \\
-0.130 \\
0.156\end{array}$ & $\begin{array}{r}0.000 \\
-0.002 \\
0.016 \\
-0.002 \\
0.018\end{array}$ & $\begin{array}{r}-0.024 \\
0.000 \\
0.007 \\
-0.025 \\
0.032\end{array}$ & 茴 \\
\hline
\end{tabular}




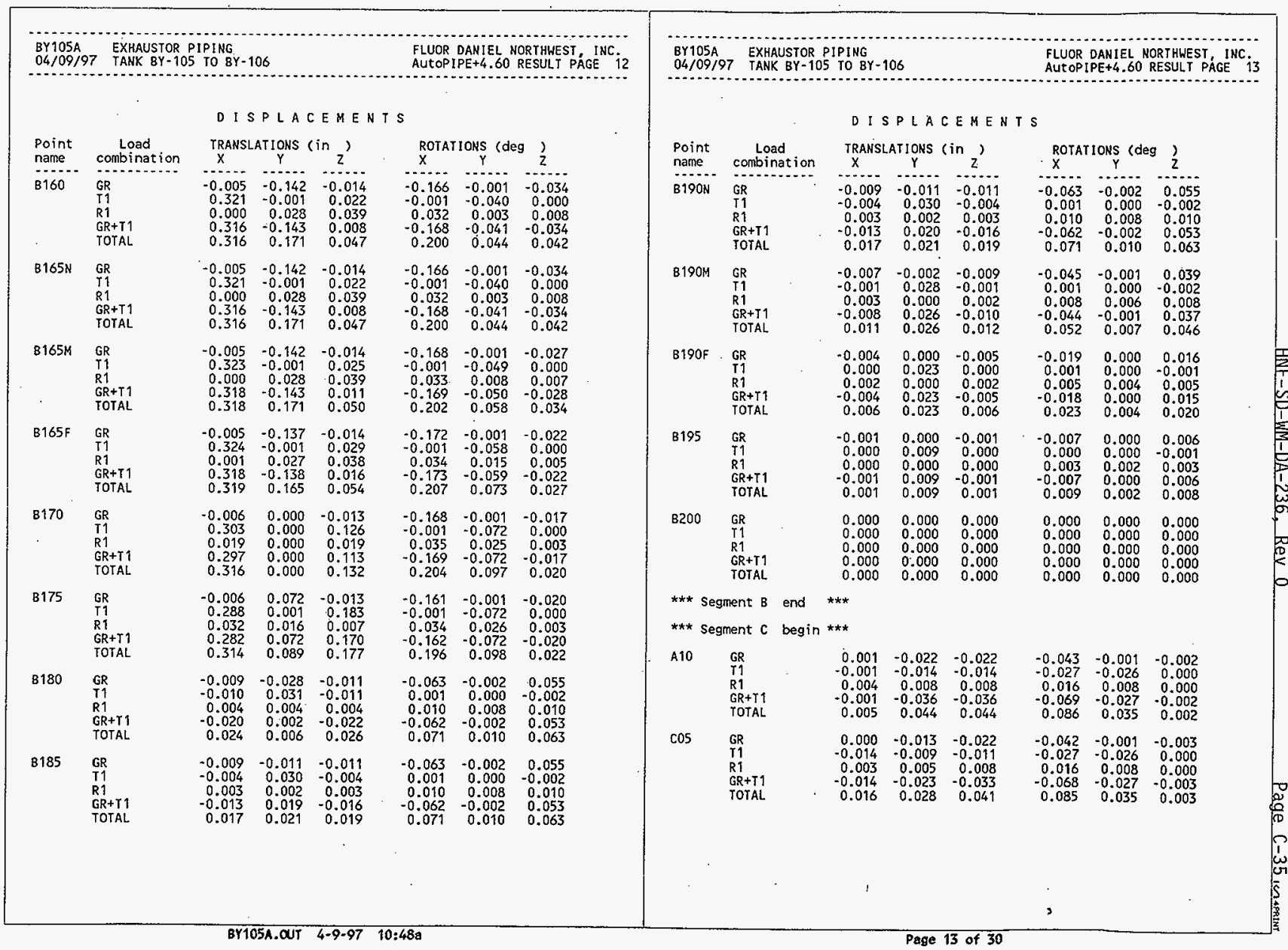




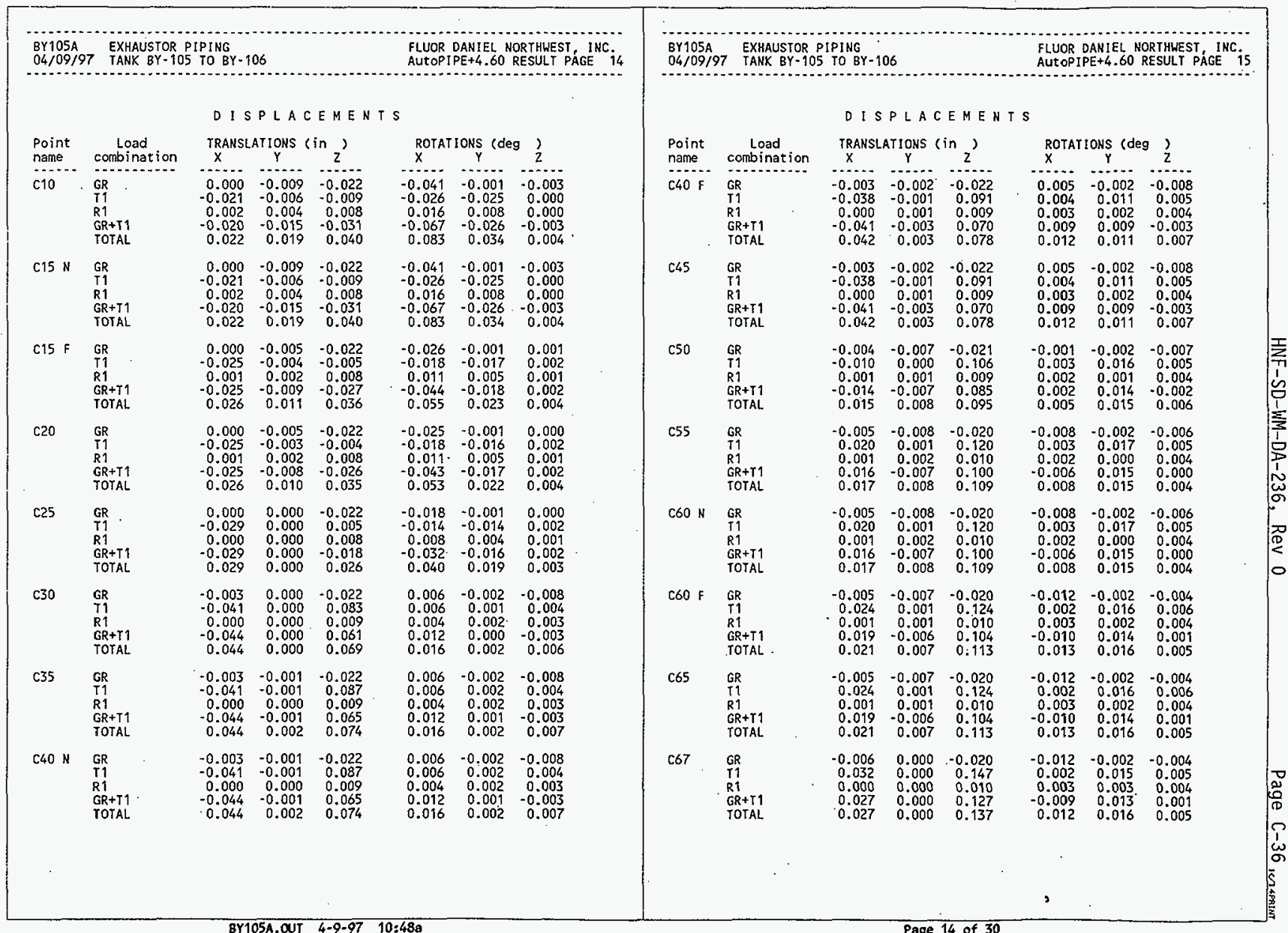




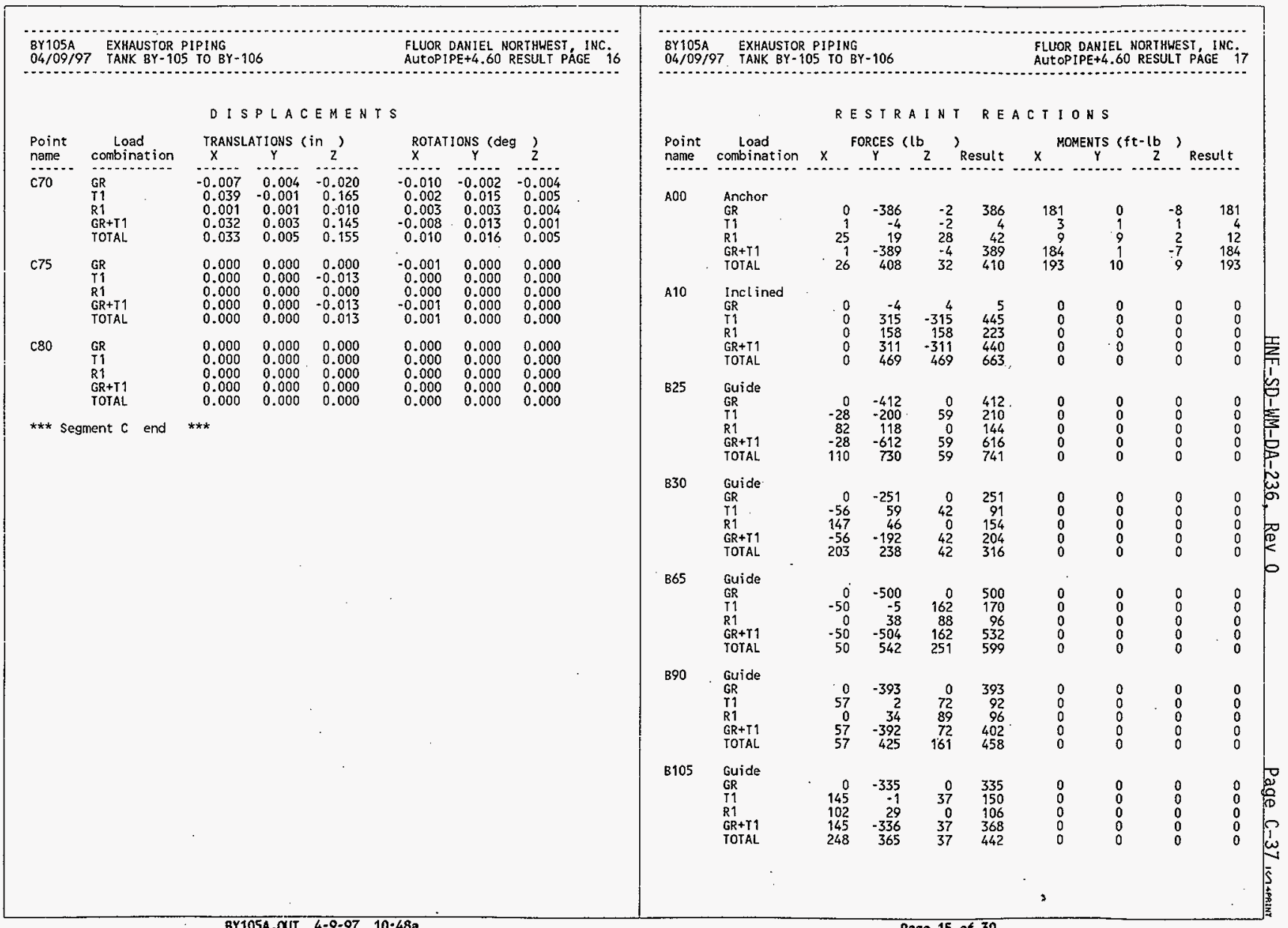




\begin{tabular}{|c|c|c|c|c|c|c|c|c|c|c|c|c|c|c|c|c|c|c|c|c|}
\hline \multicolumn{5}{|c|}{ 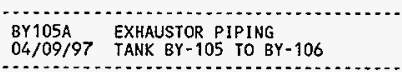 } & \multicolumn{5}{|c|}{$\begin{array}{l}\text { FLUOR DAN1EL NORY HWEST INC. } \\
\text { AUtOPIPEE } 4.60 \text { RESLLT PAGE is }\end{array}$} & \multicolumn{6}{|c|}{$\begin{array}{l}\text { BY105A } \\
04 / 09 / 97\end{array} \begin{array}{l}\text { EXHAUSTOR PIPING } \\
\text { TANK BY- } 105 \text { TO BY- } 106\end{array}$} & \multicolumn{5}{|c|}{$\begin{array}{l}\text { FLUOR DANIEL NORTHUEST, INC } \\
\text { AUTOPIPE+4.60 RESULT PAGE }\end{array}$} \\
\hline \multicolumn{10}{|c|}{ RESTRAINT REACTIONS } & \multicolumn{11}{|c|}{ RESTRAINT REACTIONS } \\
\hline $\begin{array}{l}\text { Point } \\
\text { name } \\
-. . . .\end{array}$ & $\begin{array}{l}\text { Load } \\
\text { combination }\end{array}$ & $x^{\mathrm{F}}$ & $\begin{array}{l}\text { ORCES } \\
Y\end{array}$ & $\begin{array}{l}b \\
z\end{array}$ & esult & $\mathrm{x}^{\text {MOM }}$ & $\begin{array}{l}\text { NIS }(\text { th } \\
Y . .\end{array}$ & $\begin{array}{l}-(b) \\
2 \\
2\end{array}$ & Result & $\begin{array}{l}\text { Point } \\
\text { name }\end{array}$ & $\begin{array}{l}\text { Load } \\
\text { combination }\end{array}$ & $x^{F}$ & $\begin{array}{l}\text { FORCES } \\
Y\end{array}$ & b & Result & $x^{\text {MOM }}$ & is $<\mathrm{ft}$ - & $\begin{array}{ll}\text { b } \\
z\end{array}$ & Resul & \\
\hline B110 & $\begin{array}{l}\text { Guide } \\
\text { GR } \\
\text { I1 } \\
\text { R1 } \\
\text { GR+I1 } \\
\text { TOTAL }\end{array}$ & $\begin{array}{r}0 \\
-153 \\
81 \\
-153 \\
234\end{array}$ & $\begin{array}{r}-259 \\
1 \\
32 \\
-258 \\
290\end{array}$ & $\begin{array}{r}0 \\
-38 \\
0 \\
-38 \\
38\end{array}$ & $\begin{array}{r}259 \\
158 \\
87 \\
302 \\
374\end{array}$ & $\begin{array}{l}0 \\
0 \\
0 \\
0 \\
0\end{array}$ & $\begin{array}{l}0 \\
0 \\
0 \\
0 \\
0\end{array}$ & $\begin{array}{l}0 \\
0 \\
0 \\
0 \\
0\end{array}$ & $\begin{array}{l}0 \\
0 \\
0 \\
0 \\
0\end{array}$ & $\mathrm{c25}$ & $\begin{array}{l}\text { Guide } \\
\text { GR } \\
\text { T1 } \\
\text { R1 } \\
\text { GR+T1 } \\
\text { TOTAL }\end{array}$ & $\begin{array}{r}0 \\
-59 \\
72 \\
-59 \\
131\end{array}$ & $\begin{array}{r}-435 \\
-225 \\
132 \\
-660 \\
792\end{array}$ & $\begin{array}{l}0 \\
9 \\
0 \\
9 \\
9\end{array}$ & $\begin{array}{l}435 \\
233 \\
150 \\
663 \\
803\end{array}$ & $\begin{array}{l}0 \\
0 \\
0 \\
0 \\
0\end{array}$ & $\begin{array}{l}0 \\
0 \\
0 \\
0 \\
0\end{array}$ & $\begin{array}{l}0 \\
0 \\
0 \\
0 \\
0\end{array}$ & & $\begin{array}{l}0 \\
0 \\
0 \\
0 \\
0 \\
0\end{array}$ \\
\hline B135 & $\begin{array}{l}\text { Guide } \\
\text { GR } \\
\text { T1 } \\
\text { R1 } \\
\text { GR+T1 } \\
\text { TOTAL }\end{array}$ & $\begin{array}{r}0 \\
-92 \\
0 \\
-92 \\
92\end{array}$ & $\begin{array}{r}-425 \\
-1 \\
25 \\
-426 \\
451\end{array}$ & $\begin{array}{l}-70 \\
-76 \\
-75 \\
190\end{array}$ & $\begin{array}{l}425 \\
120 \\
117 \\
442 \\
498\end{array}$ & $\begin{array}{l}0 \\
0 \\
0 \\
0 \\
0\end{array}$ & $\begin{array}{l}0 \\
0 \\
0 \\
0 \\
0\end{array}$ & $\begin{array}{l}0 \\
0 \\
0 \\
0 \\
0\end{array}$ & $\begin{array}{r}0 \\
0 \\
0 \\
0 \\
.0\end{array}$ & c30 & $\begin{array}{l}\text { Guide } \\
\text { GR } \\
\text { T1 } \\
\text { R1 } \\
\text { GR+I1 } \\
\text { TOTAL }\end{array}$ & $\begin{array}{r}0 \\
-5 \\
39 \\
-5 \\
44\end{array}$ & $\begin{array}{r}-145 \\
62 \\
42 \\
-82 \\
124\end{array}$ & $\begin{array}{c}0 \\
11 \\
0 \\
11 \\
11\end{array}$ & $\begin{array}{r}145 \\
63 \\
57 \\
83 \\
132\end{array}$ & $\begin{array}{l}0 \\
0 \\
0 \\
0 \\
0\end{array}$ & $\begin{array}{l}0 \\
0 \\
0 \\
0 \\
0\end{array}$ & $\begin{array}{l}0 \\
0 \\
0 \\
0 \\
0\end{array}$ & & $\begin{array}{l}0 \\
0 \\
0 \\
0 \\
0 \\
0\end{array}$ \\
\hline B145 & $\begin{array}{l}\text { Guide } \\
\text { GR } \\
\text { T1 } \\
\text { R1 } \\
\text { GR+Ti } \\
\text { OOIAL }\end{array}$ & $\begin{array}{r}0 \\
12 \\
0 \\
12 \\
12\end{array}$ & $\begin{array}{r}-444 \\
0 \\
29 \\
-443 \\
473\end{array}$ & $\begin{array}{r}0 \\
-31 \\
82 \\
-31 \\
113\end{array}$ & $\begin{array}{r}444 \\
34 \\
87 \\
445 \\
486\end{array}$ & $\begin{array}{l}0 \\
0 \\
0 \\
0 \\
0\end{array}$ & $\begin{array}{l}0 \\
0 \\
0 \\
0 \\
0\end{array}$ & $\begin{array}{l}0 \\
0 \\
0 \\
0 \\
0\end{array}$ & $\begin{array}{l}0 \\
0 \\
0 \\
0 \\
0\end{array}$ & C67 & $\begin{array}{l}\text { Guide } \\
\text { GR } \\
\text { T1 } \\
\text { R1 } \\
\text { GR+T1 } \\
\text { TOTAL }\end{array}$ & $\begin{array}{r}0 \\
10 \\
21 \\
10 \\
31\end{array}$ & $\begin{array}{r}-240 \\
-49 \\
19 \\
-244 \\
255\end{array}$ & $\begin{array}{r}0 \\
44 \\
04 \\
44 \\
44\end{array}$ & $\begin{array}{r}240 \\
45 \\
23 \\
248 \\
260\end{array}$ & $\begin{array}{l}0 \\
0 \\
0 \\
0 \\
0\end{array}$ & $\begin{array}{l}0 \\
0 \\
0 \\
0 \\
0\end{array}$ & $\begin{array}{l}0 \\
0 \\
0 \\
0 \\
0\end{array}$ & & $\begin{array}{l}0 \\
0 \\
0 \\
0 \\
0\end{array}$ \\
\hline 8145 & $\begin{array}{l}\text { Line Stp } \\
\text { GR } \\
\text { T1 } \\
\text { R1 } \\
\text { GRT1 } \\
\text { TOTAL }\end{array}$ & $\begin{array}{r}0 \\
0 \\
150 \\
0 \\
150\end{array}$ & $\begin{array}{l}0 \\
0 \\
0 \\
0 \\
0\end{array}$ & $\begin{array}{l}0 \\
0 \\
0 \\
0 \\
0\end{array}$ & $\begin{array}{r}0 \\
0 \\
150 \\
0 \\
150\end{array}$ & $\begin{array}{l}0 \\
0 \\
0 \\
0 \\
0\end{array}$ & $\begin{array}{l}0 \\
0 \\
0 \\
0 \\
0\end{array}$ & $\begin{array}{l}0 \\
0 \\
0 \\
0 \\
0\end{array}$ & $\begin{array}{l}0 \\
0 \\
0 \\
0 \\
0\end{array}$ & $\mathrm{c} 80$ & $\begin{array}{l}\text { Anchor } \\
\text { GR } \\
\text { T1 } \\
\text { R1 } \\
\text { GR+T1 } \\
\text { OOTAL }\end{array}$ & $\begin{array}{l}0 \\
2 \\
5 \\
1 \\
7\end{array}$ & $\begin{array}{r}-94 \\
0 \\
3 \\
-94 \\
98\end{array}$ & $\begin{array}{l}-2 . \\
14 \\
6 \\
13 \\
19\end{array}$ & $\begin{array}{c}94 \\
14 \\
9 \\
95 \\
100\end{array}$ & $\begin{array}{r}-125 \\
0 \\
3 \\
-125 \\
128\end{array}$ & $\begin{array}{r}1 \\
-3 \\
-6 \\
-3 \\
8\end{array}$ & $\begin{array}{r}-3 \\
3 \\
2 \\
1 \\
1 \\
3\end{array}$ & & $\begin{array}{r}25 . \\
5 \\
27 \\
25 \\
28\end{array}$ \\
\hline B155 & $\begin{array}{l}\text { Guide } \\
\text { GR } \\
T 1 \\
\text { R1 } \\
\text { GR+r1 } \\
\text { TOTAL }\end{array}$ & $\begin{array}{l}0 \\
90 \\
0 \\
90 \\
90\end{array}$ & $\begin{array}{r}-518 \\
-1 \\
31 \\
-518 \\
549\end{array}$ & $\begin{array}{r}-0 \\
-13 \\
-67 \\
-13 \\
80\end{array}$ & $\begin{array}{r}518 \\
91 \\
74 \\
526 \\
563\end{array}$ & $\begin{array}{l}0 \\
0 \\
0 \\
0 \\
0\end{array}$ & $\begin{array}{l}0 \\
0 \\
0 \\
0 \\
0\end{array}$ & $\begin{array}{l}0 \\
0 \\
0 \\
0 \\
0\end{array}$ & $\begin{array}{l}0 \\
0 \\
0 \\
0 \\
0\end{array}$ & & . & & & & & & & & & \\
\hline 8170 & $\begin{array}{l}\text { Guide } \\
\text { GR } \\
\text { T1 } \\
\text { R1 } \\
\text { GR+T1 } \\
\text { TOTAL }\end{array}$ & $\begin{array}{r}10 \\
.04 \\
.34 \\
104 \\
138\end{array}$ & $\begin{array}{r}-359 \\
2 \\
15 \\
-358 \\
373\end{array}$ & $\begin{array}{r}0 \\
8 \\
34 \\
8 \\
42\end{array}$ & $\begin{array}{l}359 \\
104 \\
51 \\
373 \\
400\end{array}$ & $\begin{array}{l}0 \\
0 \\
0 \\
0 \\
0\end{array}$ & $\begin{array}{l}0 \\
0 \\
0 \\
0 \\
0\end{array}$ & $\begin{array}{l}0 \\
0 \\
0 \\
0 \\
0\end{array}$ & $\begin{array}{l}0 \\
0 \\
0 \\
0 \\
0\end{array}$ & & & & & & & & & & & \\
\hline .8200 & $\begin{array}{l}\text { Anchor } \\
G R \\
T 1 \\
R 1 \\
\text { GR+T1 } \\
\text { TOTAL }\end{array}$ & $\begin{array}{r}0 \\
22 \\
87 \\
22 \\
108\end{array}$ & $\begin{array}{r}-835 \\
-1 \\
72 \\
-836 \\
908\end{array}$ & $\begin{array}{r}0 \\
18 \\
84 \\
18 \\
102\end{array}$ & $\begin{array}{l}835 \\
28 \\
141 \\
837 \\
920\end{array}$ & $\begin{array}{r}-706 \\
57 \\
286 \\
-649 \\
.935\end{array}$ & $\begin{array}{r}0 \\
-6 \\
113 \\
-66 \\
119\end{array}$ & $\begin{array}{r}600 \\
-70 \\
297 \\
.530 \\
827\end{array}$ & $\begin{array}{r}927 \\
91 \\
427 \\
838 \\
1254\end{array}$ & & & . & & & & & & & & \\
\hline & & . & & & & & & & & & & & & & & , & & & & \\
\hline
\end{tabular}




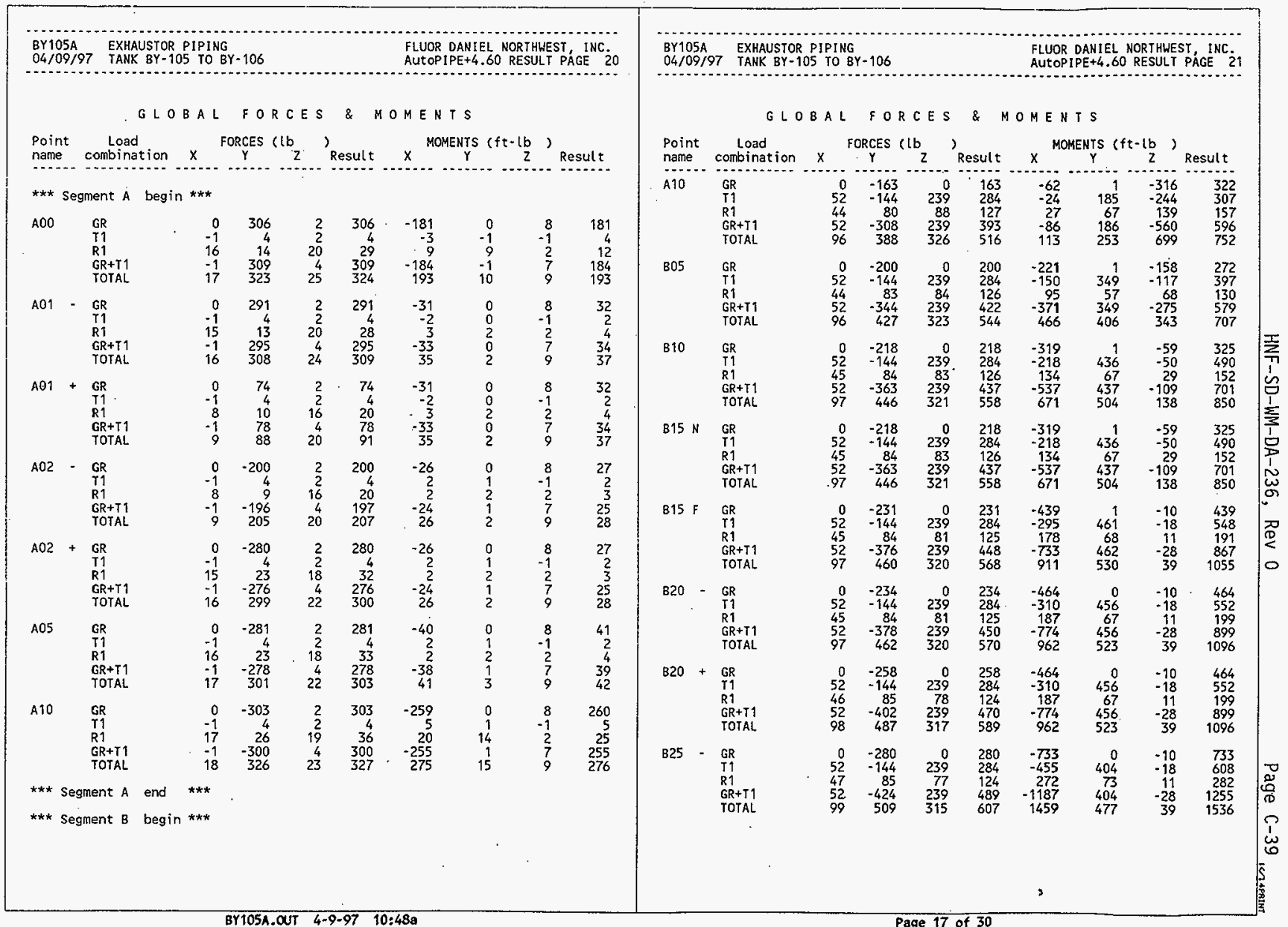




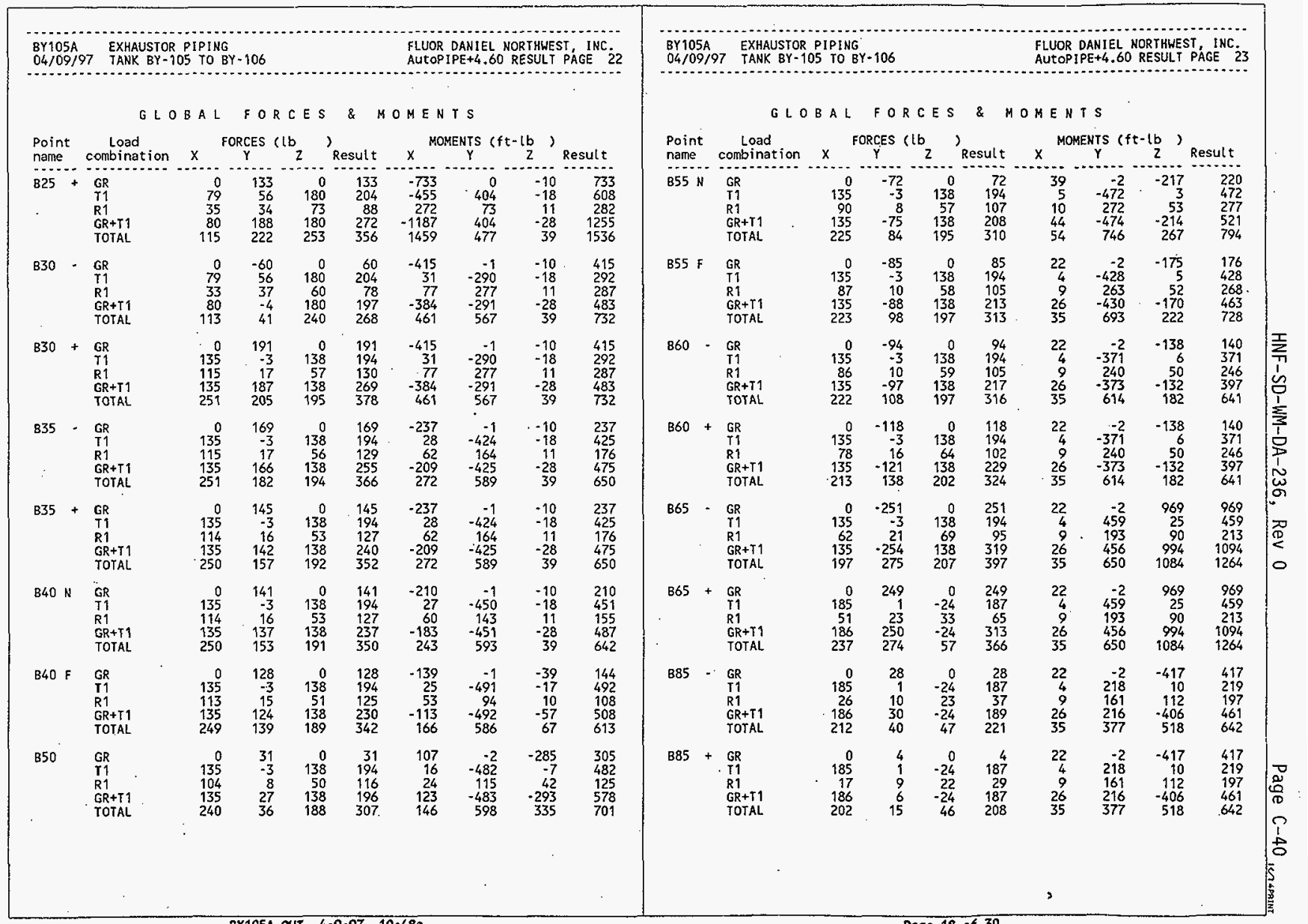




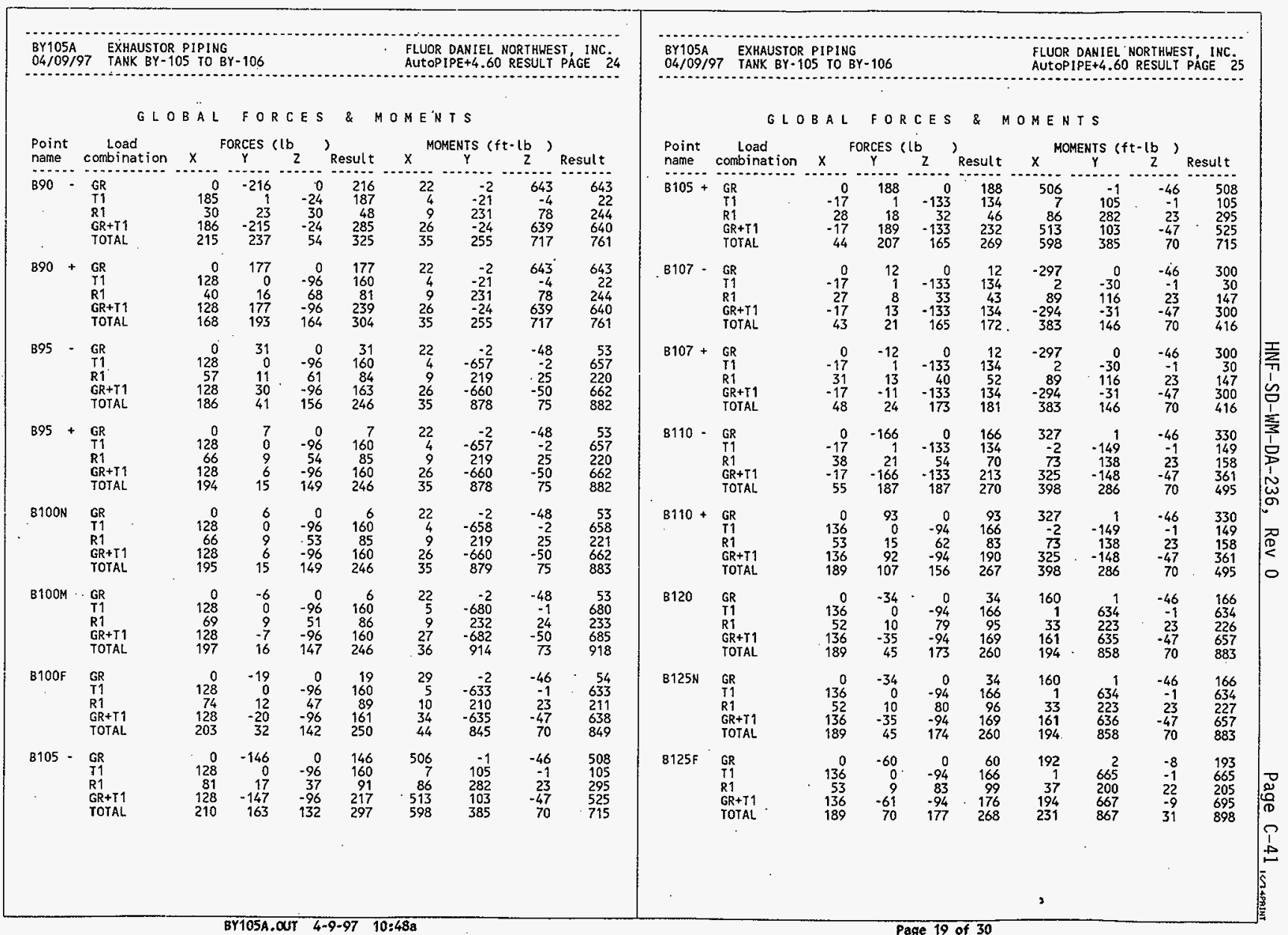




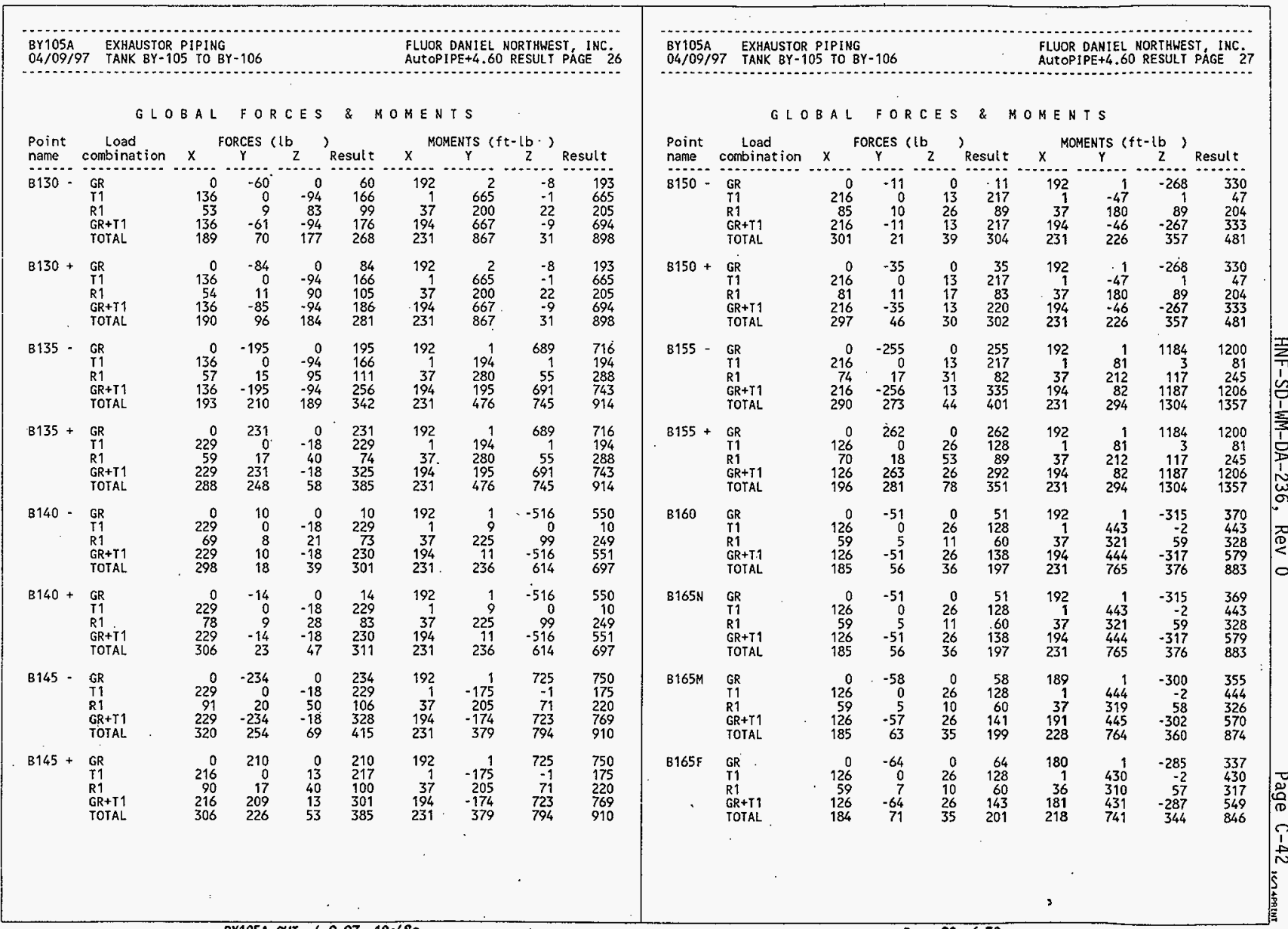




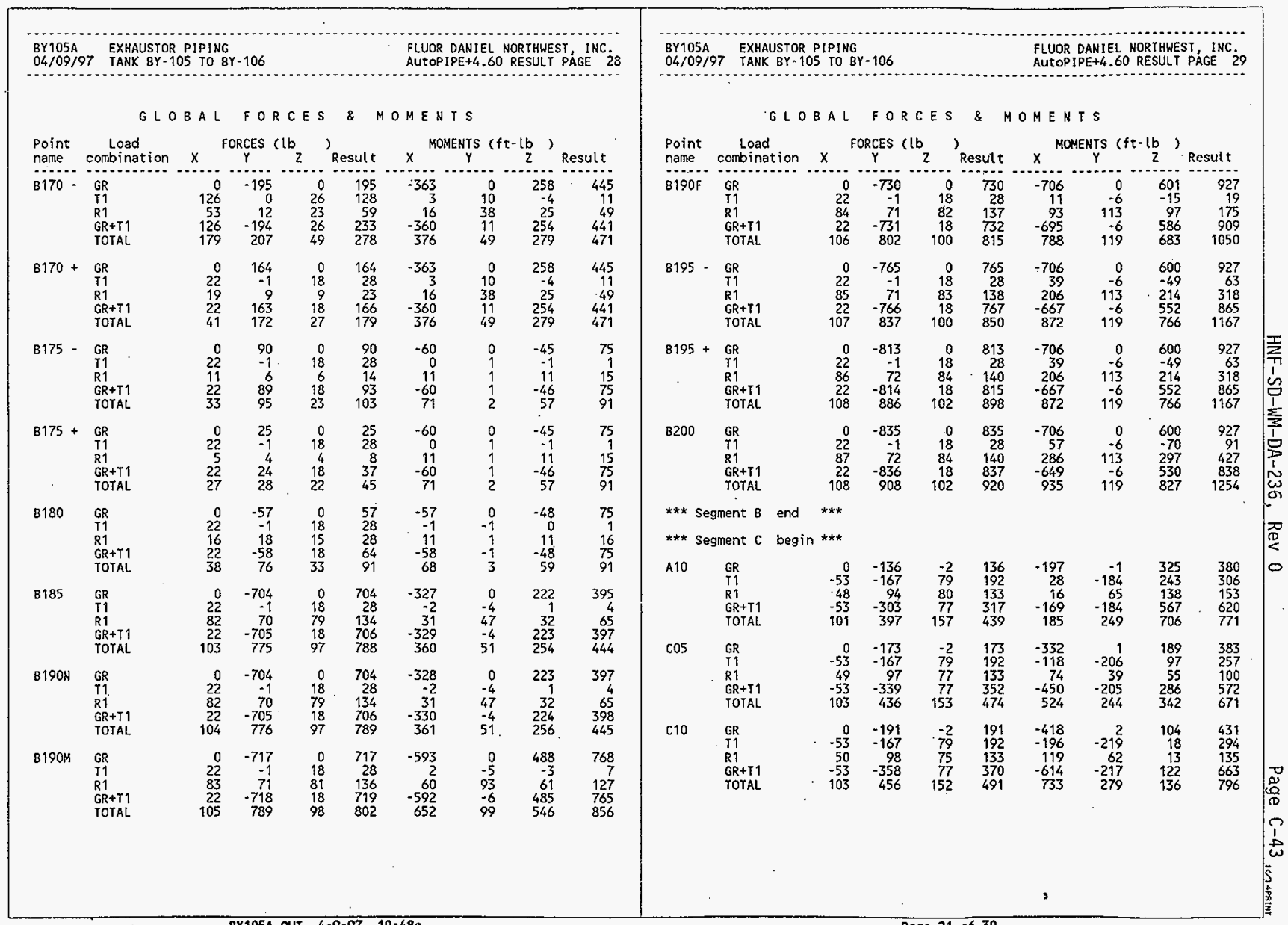




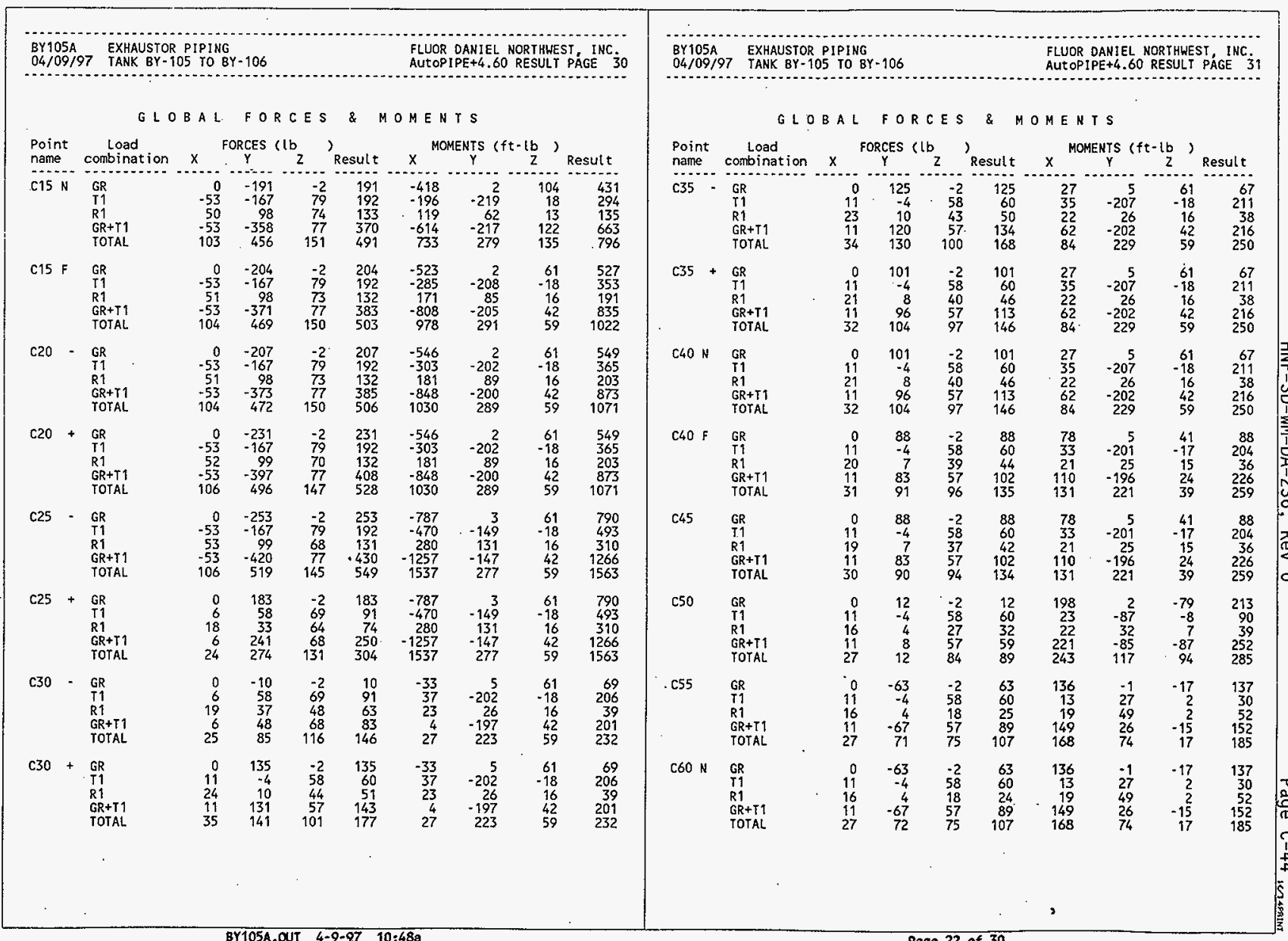




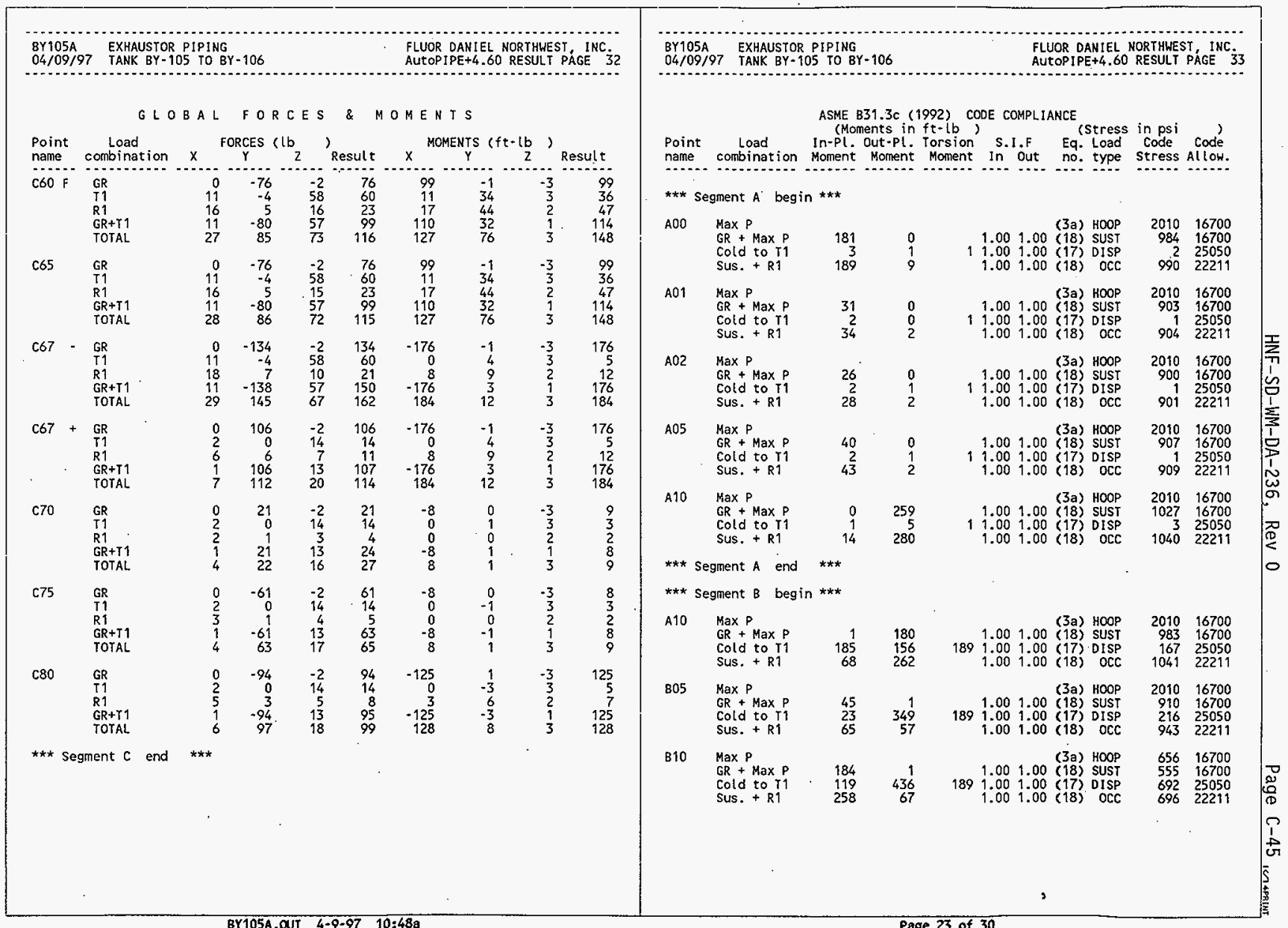




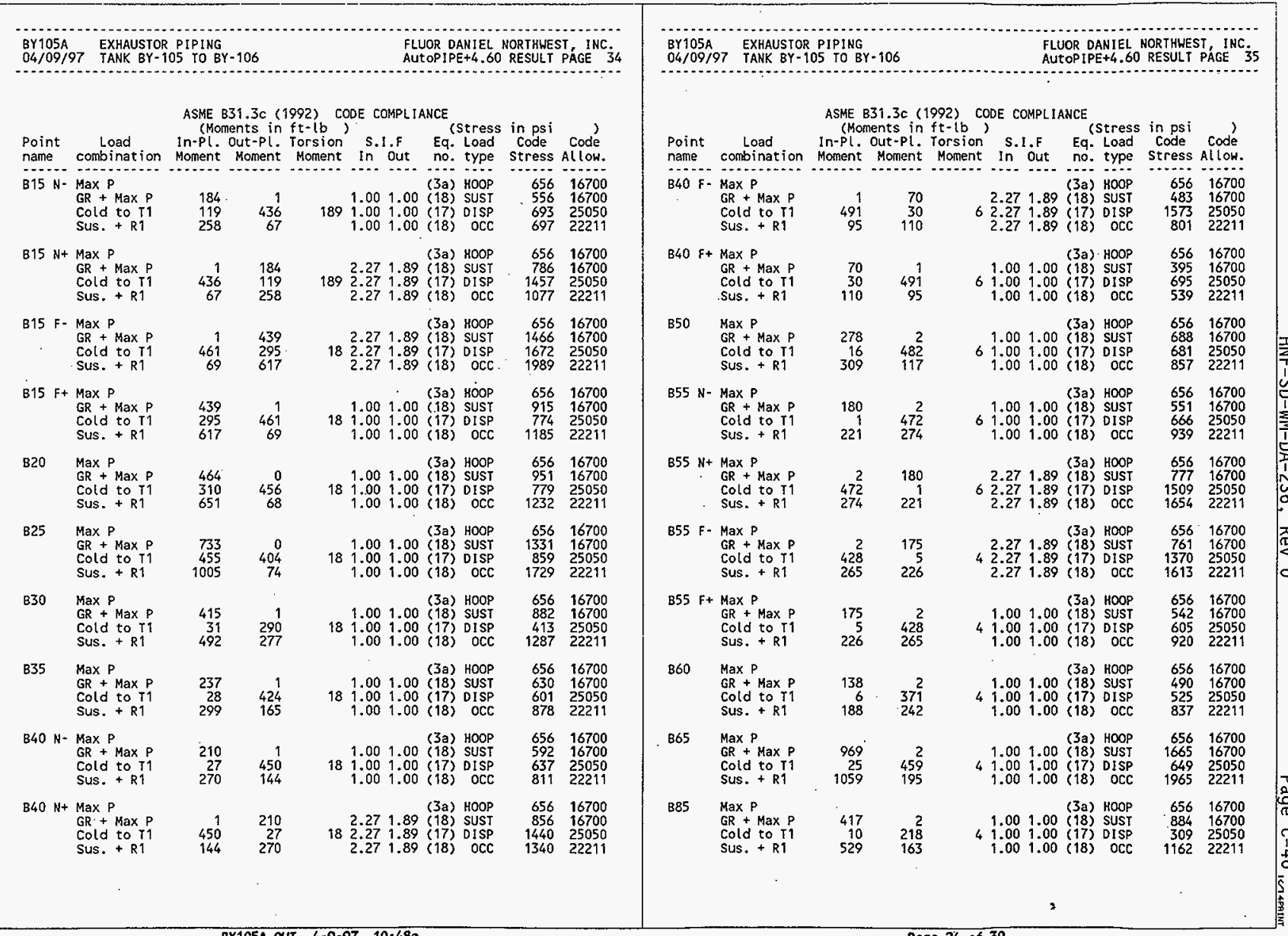




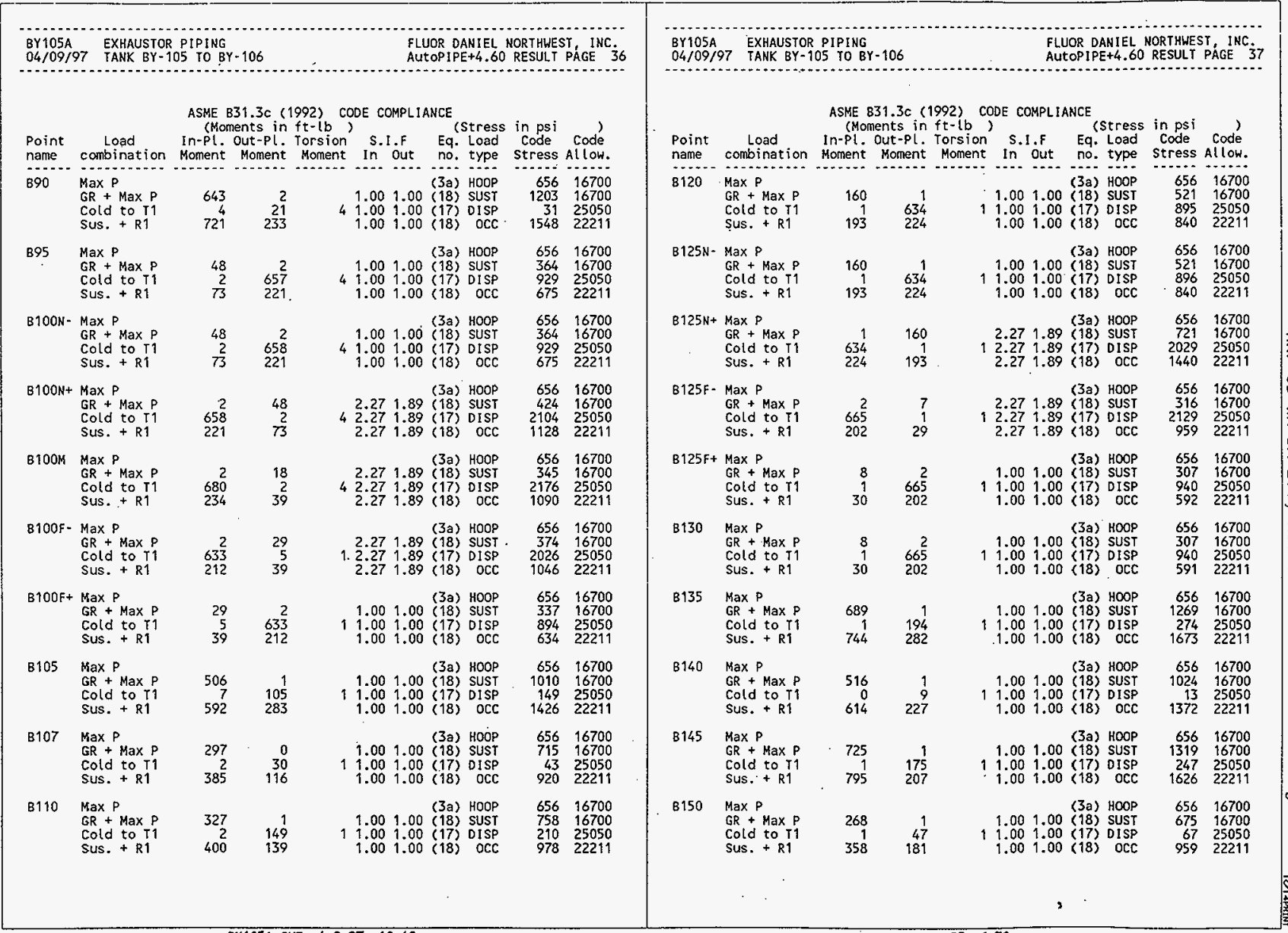




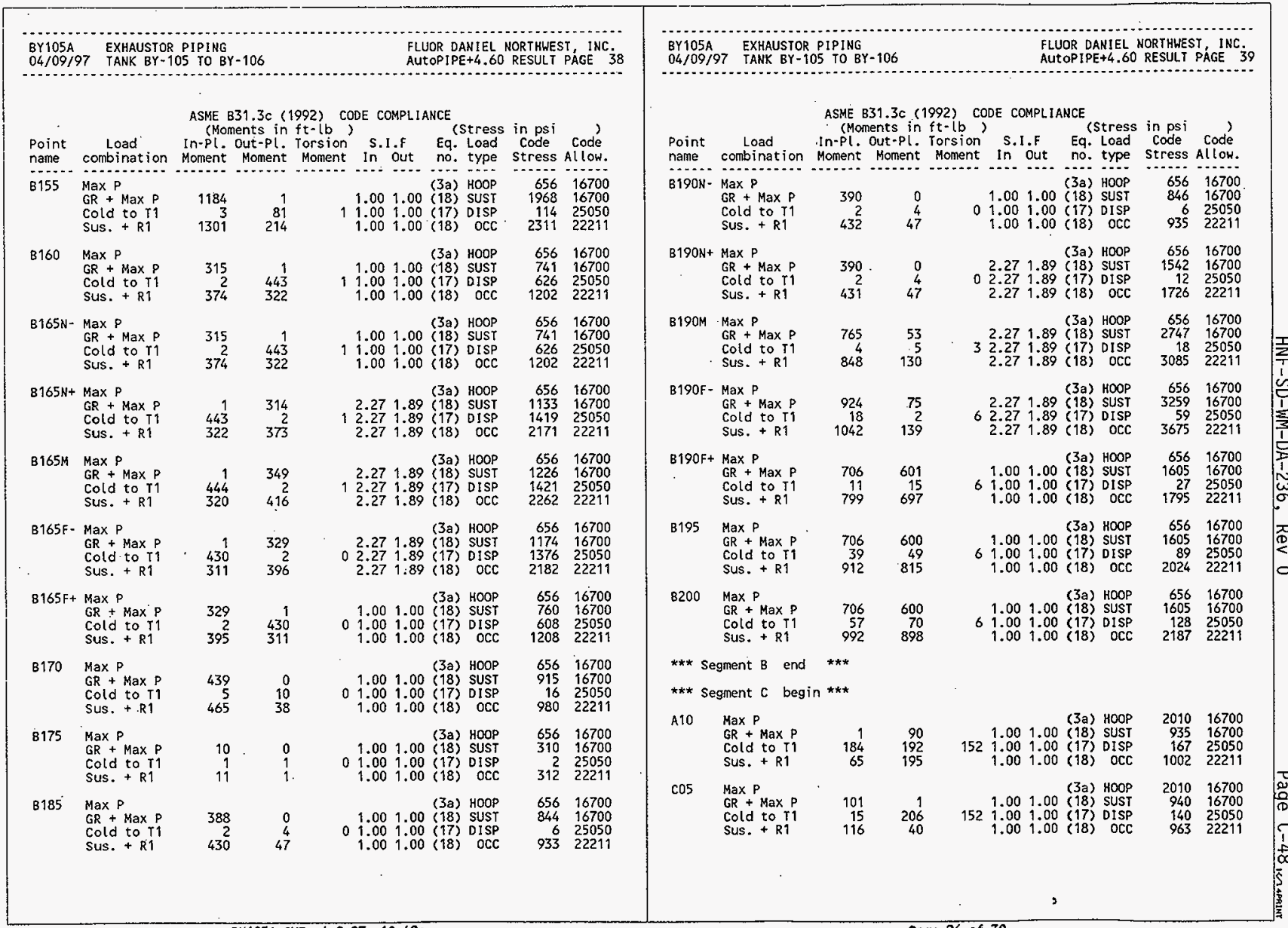




EXHAUSTOR PIPING
$04 / 09 / 97$ TANK BY-105 TO BY-106

ASME B31.3c (1992) CODE COMPLIANCE

(Moments in $\mathrm{ft}-\mathrm{lb}$ ) (Stress in psi point Load In-Pl. Out-Pl. Torsion S.1.F Eq. Load Code Code name combination Moment Moment Moment In Out no. type stress Allow. C10 Max $\begin{array}{lllllll}\text { GR + Max P } & 222 & 2 & 1.001 .00 \text { (3a) HOOP } & 65616700 \\ \text { SUST } & 609 & 16700\end{array}$ Cold to $P 11 \quad 226 \quad 219 \quad 1521.001 .00$ (17) OISP

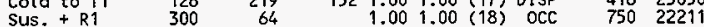

C15 N- Max $P$ $\mathrm{GR}+\operatorname{Max} \mathrm{P}$ Cold to T1 Sus. + R1

C15 $N+\operatorname{Max} P$ GR + Max P Cold to T1 Sus. + R1

C15 F- $\operatorname{Max} P$ GR + Max ? Cold.to Th Sus. + R1

C15 F+ Max $P$ $\mathrm{GR}+\operatorname{Max} P$ cold to II Sus. + R1

C20 Max P $G R+\operatorname{Max} P$ Cold to T1 Sus. $+R 1$

C25 Max P GR + Max ? Cold to $T 1$ Sus. + R1

C30 Max $P$ $\mathrm{GR}+\operatorname{Max} P$ Cold to $T 1$ sus. + R

C35 Max P $\mathrm{GR}+\operatorname{Max}$ Cold to $T 1$ Sus. + R1

C40 N- Max P GR + Max $P$ Cold to T1
sus. $+\mathrm{R} 1$

\section{(3a) HOOP}

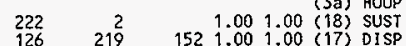
$300 \quad 64 . \quad 1.001 .00(18)$ OCC

(3a) $\mathrm{HOOP}$ $\begin{array}{rr}2 & 222 \\ 219 & 126\end{array}$ 2.271 .89 (18) SUST $\begin{array}{ll}152 & 2.27 \\ 2.27 & 1.89(17) \text { DISP }\end{array}$

(3a) HOOP $\begin{array}{rr}2 & 523 \\ 208 & 285 \\ 88 & 694\end{array}$ 2.271 .89 (18) SUST 182.271 .89 (17) $01 \mathrm{SP}$

1.001 .00 (3a) HOOP

$\begin{array}{rr}523 & 2 \\ 285 & 208 \\ 694 & 88\end{array}$ 181.001 .00 (17) DISP

1.001 .00 (3a) HOOP

$\begin{array}{rr}546 & 2 \\ 303 & 202 \\ 727 & 92\end{array}$ $181.001 .00(17)$ DISP

\section{(3a) HOOP}

$\begin{array}{rr}787 & 3 \\ 470 & 149 \\ 1067 & 134\end{array}$

$181.001 .00(18)$ SUST $1.001 .00(18)$ OCC

\section{(3a) $\mathrm{HOOP}$}

$\begin{array}{rr}33 & 5 \\ 37 & 202 \\ 56 & 32\end{array}$

1.001 .00 (18) SUST $\begin{array}{rl}18 & 1.001 .00(17) \text { OISP } \\ 1.00 & 1.00(18) \text { OCC }\end{array}$ (3a) HOOP 1.001 .00 (18) SUST $181.001 .00(17)$ DISP 1.001 .00 (18) OCC
(3a) HOOP $\begin{array}{lr}27 & 5 \\ 35 & 207 \\ 49 & 32\end{array}$ 1.001 .00 (18) SUST
1.001 .00 (17) DISP $1.001 .00(18)$ OCC
$656 \quad 16700$ 61016700 $416 \quad 25050$ $656 \quad 16700$ $\begin{array}{ll}656 & 16700 \\ 888 & 16700\end{array}$ 805
1175 22050

$656 \quad 16700$ $1691 \quad 16700$ 101025050

$656 \quad 16700$ 103516700 $\begin{array}{rr}499 & 25050 \\ 1304 & 22211\end{array}$

65616700 $1066 \quad 16700$ 51525050

65616700 140816700 $\begin{array}{rr}697 & 25050 \\ 1844 & 22211\end{array}$

65616700 34316700 $291 \quad 25050$

$656 \quad 16700$ 33516700 29825050

65616700 $\begin{array}{ll}335 & 16700\end{array}$ $\begin{array}{ll}298 & 25050 \\ 384 & 22211\end{array}$
BY105A EXHAUSTOR PIPING

$04 / 09 / 97$ TANK BY -105 TO $\mathrm{BY} \cdot 106$

FLUOR DANIEL NORTHWEST, INC. AUTOPIPE+4.60 RESULT PÁGE 41

ASME B31.3C (1992) CODE COMPLIANCE

(Moments in $\mathrm{ft}-\mathrm{lb}$ )
point Load In-Pl. Out-Pl. Torsion s.l.F Eq. Load code code name combination Moment Moment Moment in out no. type stress Allow. $\mathrm{C} 40 \mathrm{~N}+\mathrm{MaX}$ $\begin{array}{llllll}\text { Cold to T1 } & 207 & 35 & 192.27 & 1.89 \text { (17) DISP } \\ \text { Sus t R1 } & 32 & 49 & 2.271 .89 \text { (18) OCC }\end{array}$

C40 F- Max $P$ $G R+$ Max cold to T $20 \stackrel{5}{30}$ Sus. +

C40 F+ Max P $G R+\operatorname{Max} P$ Cold to $T$ Sus. + R1

C45 $\operatorname{Max} P$ $G R+\operatorname{Max} P$ Cold to $Y$
sus. +81

C50 $\operatorname{Max} P$ $G R+\operatorname{Max}$ Cold to I1

C55 Max P $G R+M a x$ Cold to I Sus. + R1

C60 N- Max P $\mathrm{GR}+\operatorname{Max}$
Cold to Sus. +

C60 N+ Max P $G R+\operatorname{Max} P$ Cold to $T 1$
Sus. $+R 1$

C60 F- Max P $\mathrm{GR}+\operatorname{Max} P$ Cold to $Y$

C60 F+ Max P $\mathrm{GR}+\operatorname{Max} \mathrm{P}$ Sus. + R1 $\begin{array}{ll}2.27 & \text { (3a) HOOP } \\ 1.89 \text { (18) SUST }\end{array}$ 102.271 .89 (17) DISP (3a) HOOP 1.001 .00 (18) SUST $\begin{array}{ll}11 & 1.001 .00(17) \text { DISP } \\ 1.001 .00(18) \text { OCC }\end{array}$

(3a) $\mathrm{HOOP}$

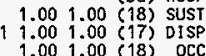
1.00 1.00 (18) (3a) HOOP 1.001 .00 (18) SUST $11.001 .00(17)$ DISP (3a) HOOP 1.001 .00 (18) SUST $1.001 .00(17)$ DISP
$1.001 .00(18)$ OCC (3a) HOOP $1.001 .00(18)$ SUST

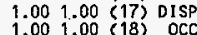
(3a) HOOP 2.271 .89 (18) SUST $\begin{array}{lll}2.27 & 1.89 & (17) \\ 2.27 & 1.89 & \text { DISP }\end{array}$ (3a) $\mathrm{HOOP}$ 2.271 .89 (18) SUST

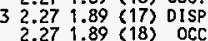
$\begin{array}{llll}1.00 & 1.00 & (3 a) & \text { HOOP } \\ 3 & 18) & \text { SUSI } \\ 1.00 & 1.00(17) & \text { DISP }\end{array}$ 1.001 .00 (18) OCC
65616700 $\begin{array}{ll}372 & 16700 \\ 671 & 25050\end{array}$ $\begin{array}{ll}671 & 25050 \\ 475 & 22211\end{array}$ $656 \quad 16700$ 36916700 $\begin{array}{ll}650 & 25050 \\ 468 & 22211\end{array}$ 65616700 33316700 $\begin{array}{ll}289 & 25050 \\ 380 & 22211\end{array}$ $656 \quad 16700$ 33316700 $380 \quad 22211$ $656 \quad 16700$ $\begin{array}{ll}573 & 16700 \\ 127 & 25050\end{array}$ 62522211 65616700 44916700 52122211

65616700 44916700 52122211

65616700 58516700 $746 \quad 22211$

$656 \quad 16700$ 55916700 70722211 65616700 $\begin{array}{rr}435 & 16700 \\ 50 & 25050\end{array}$ $502 \quad 22211$ 


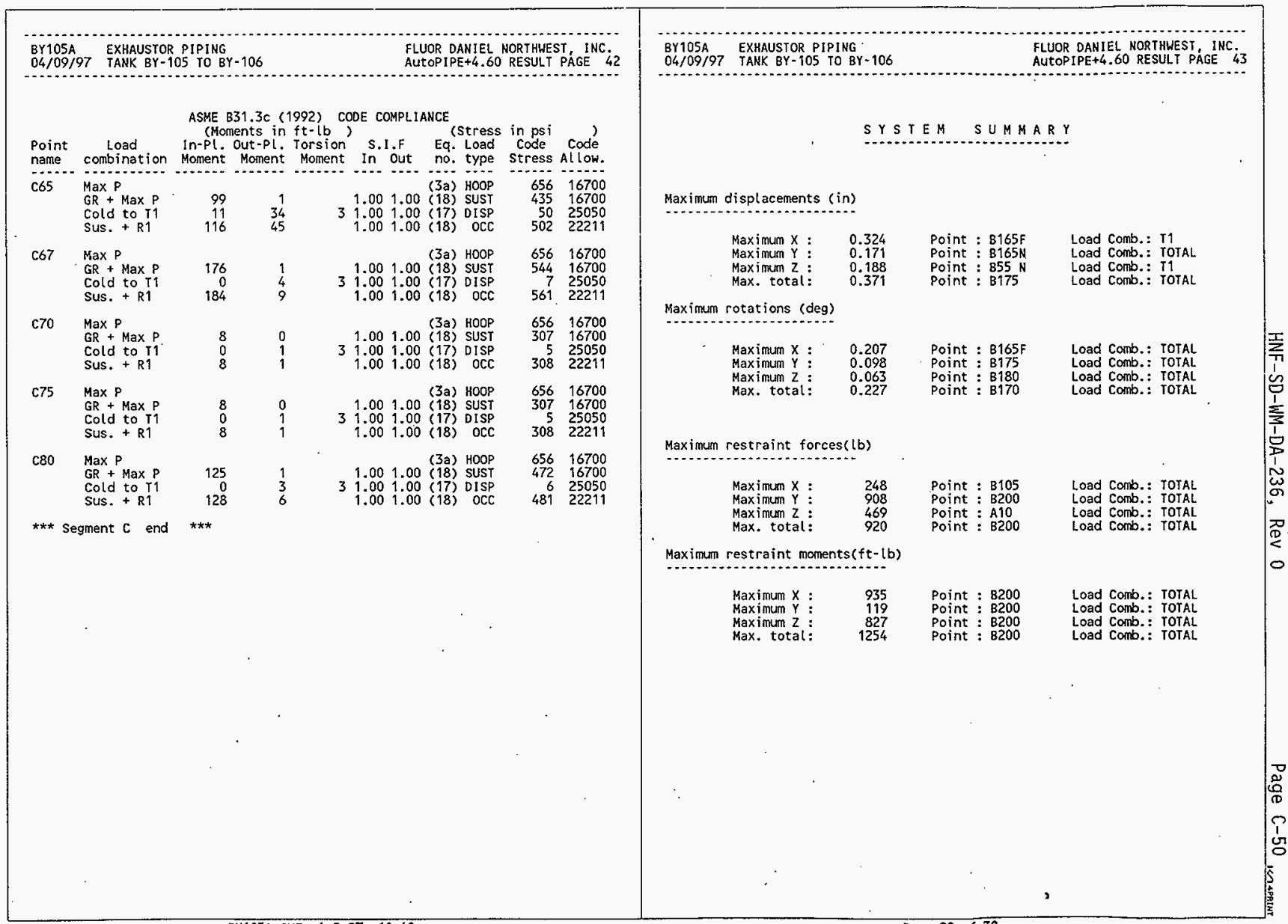




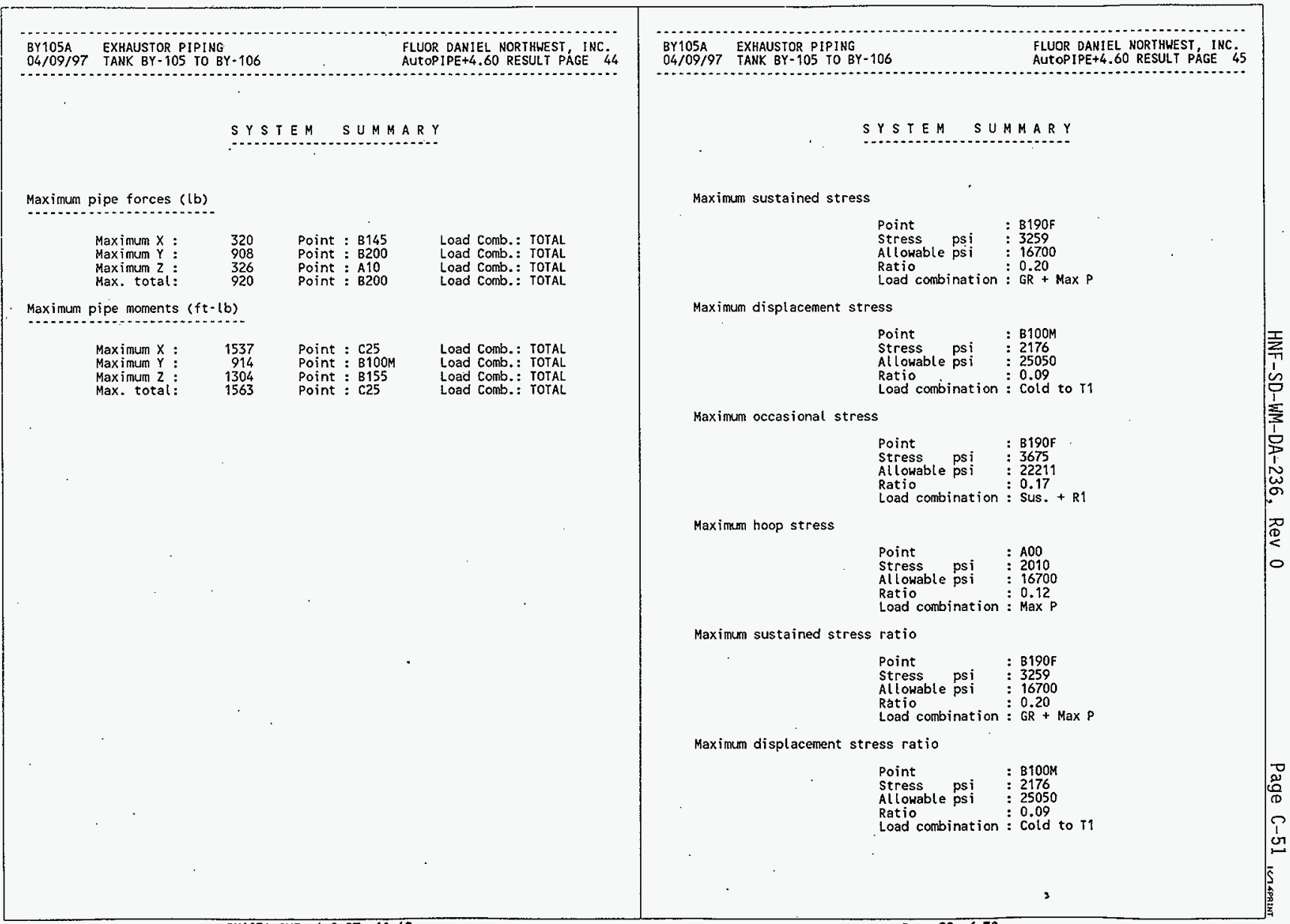




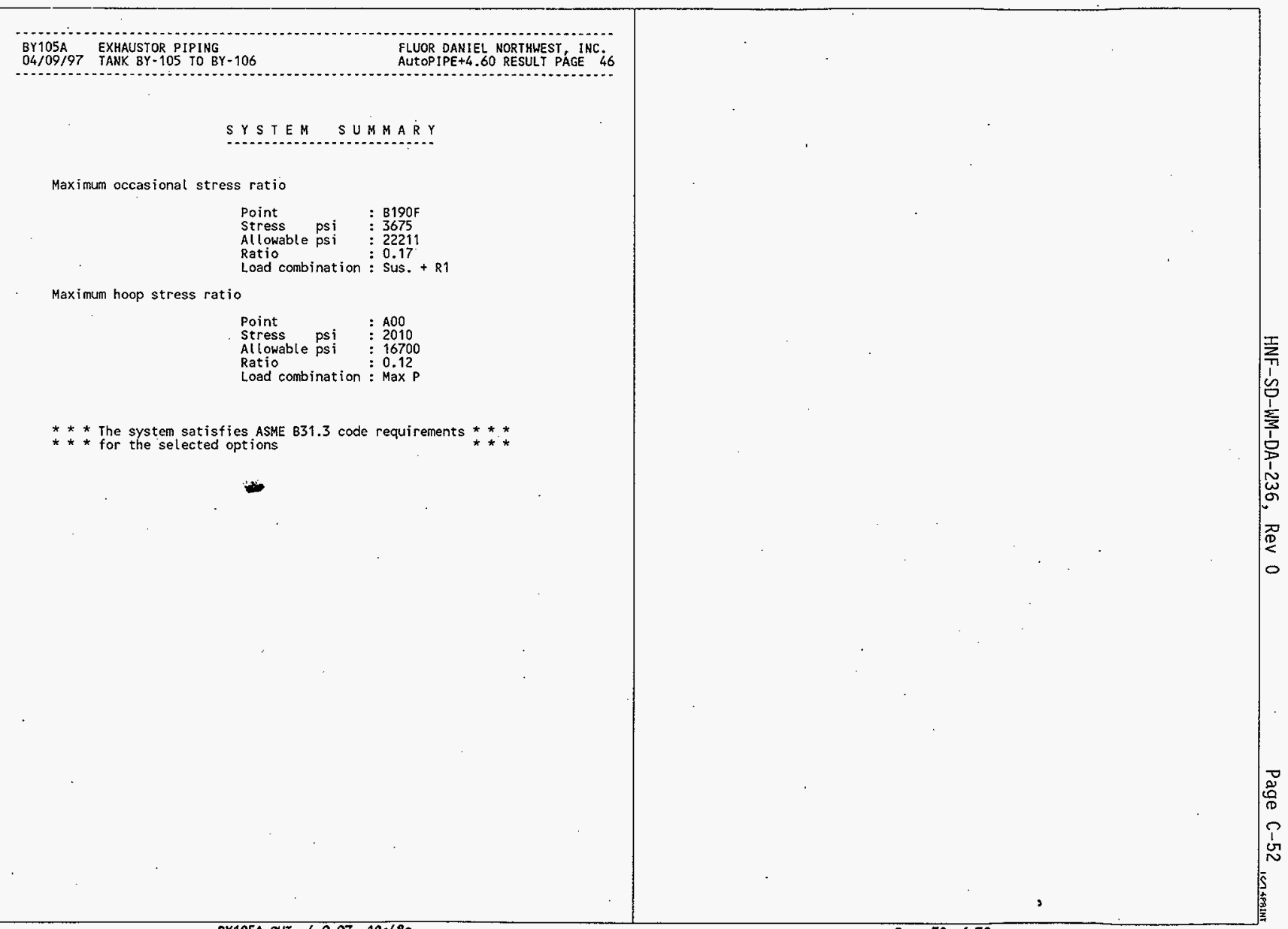



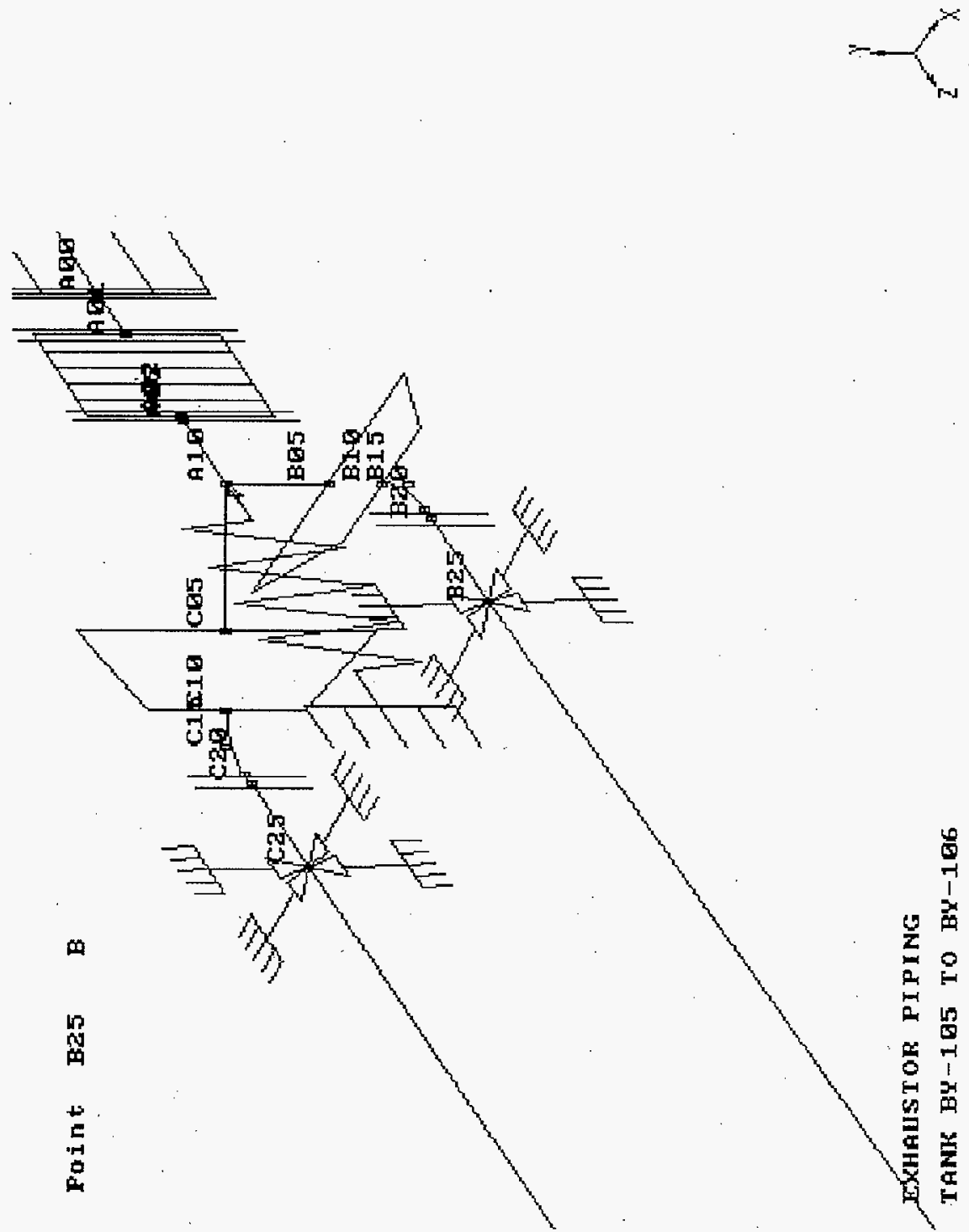


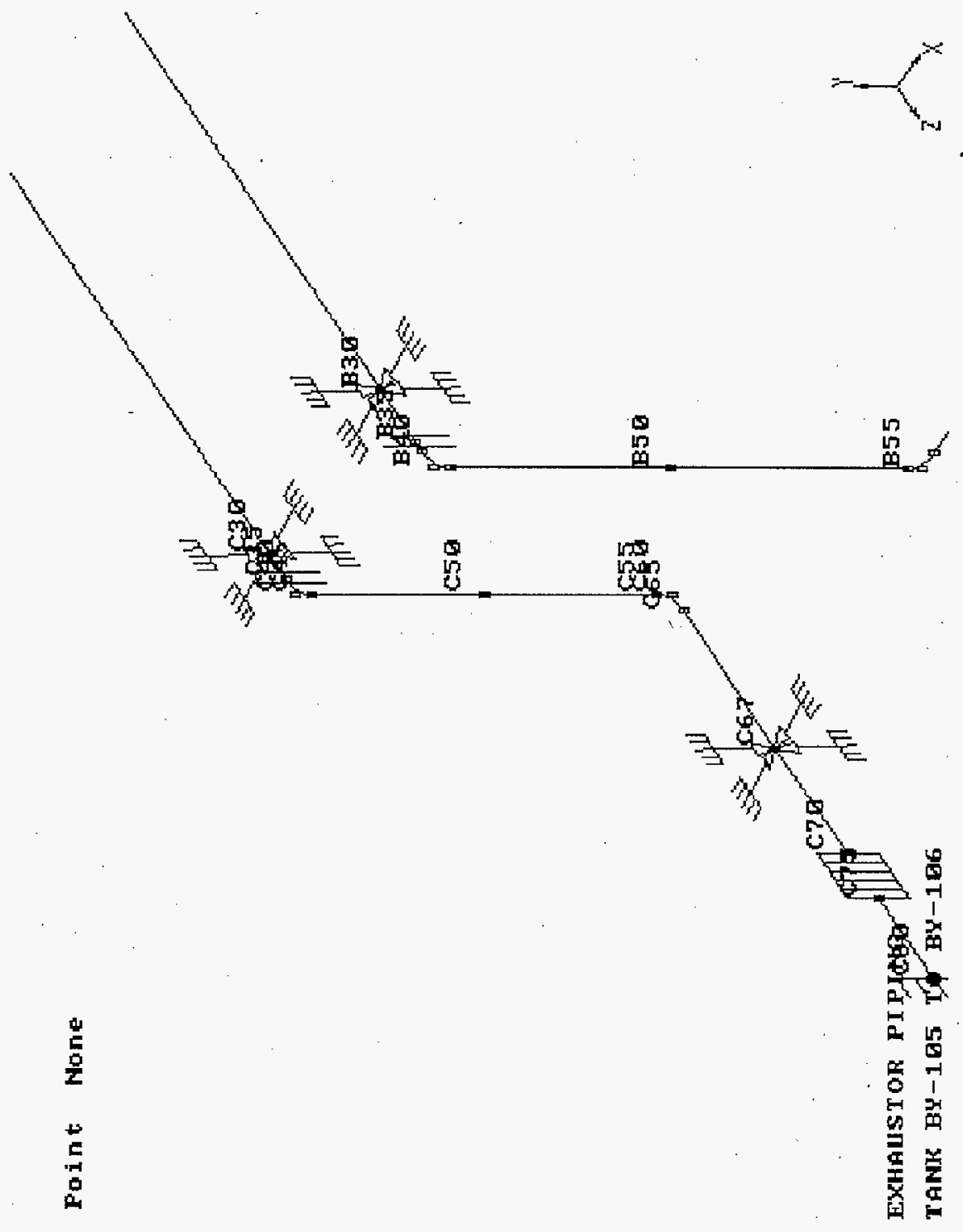



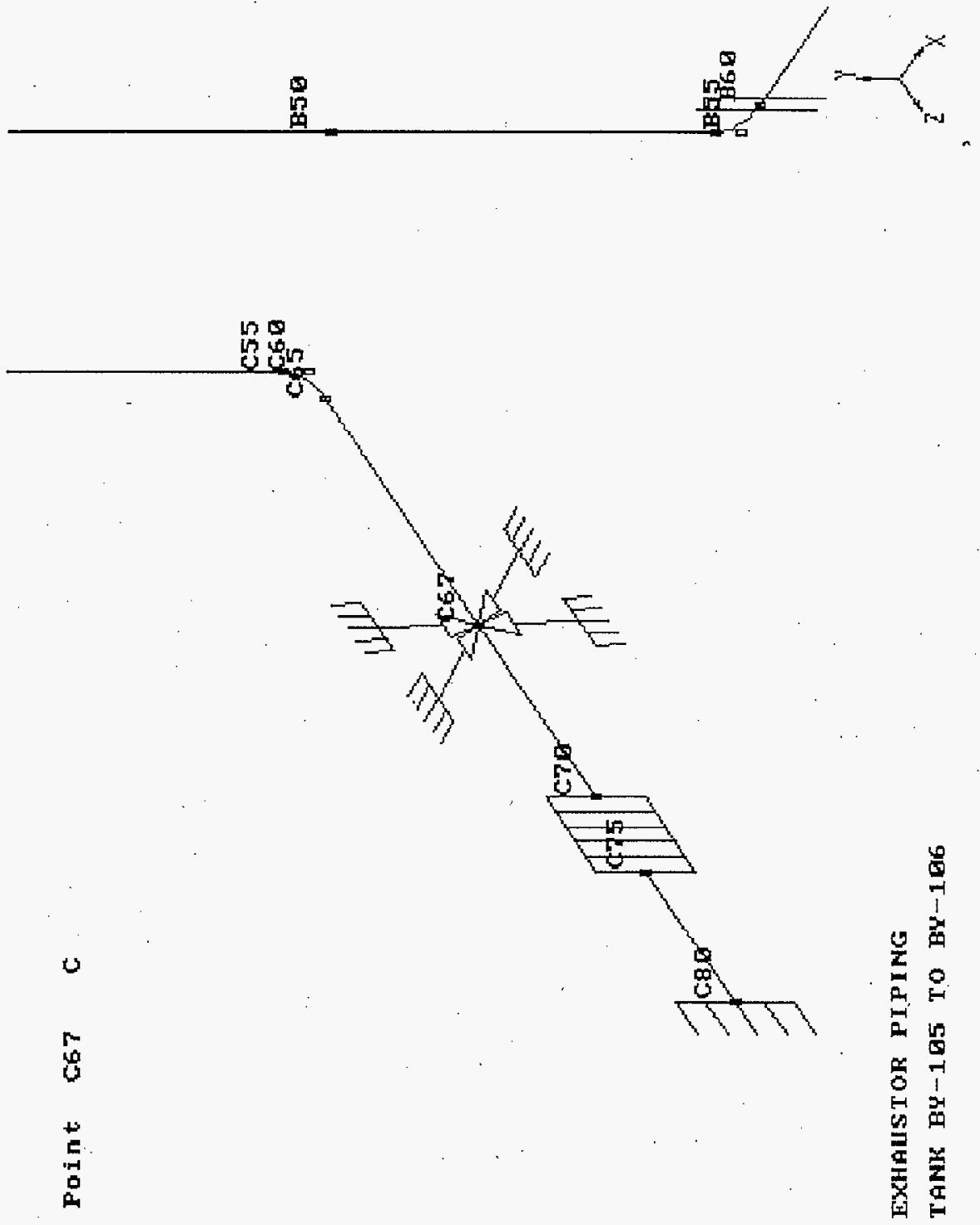

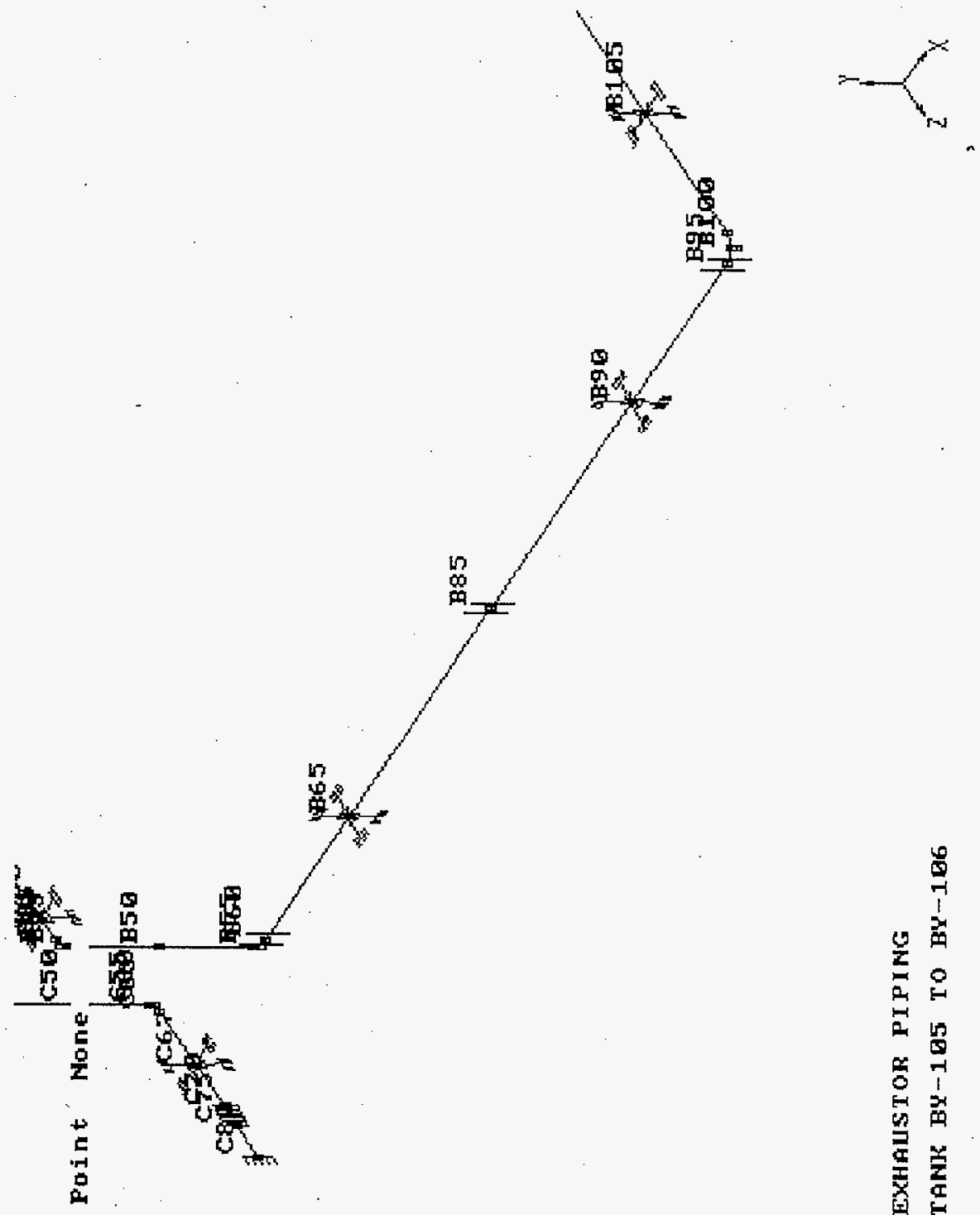

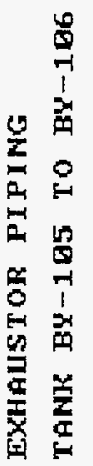




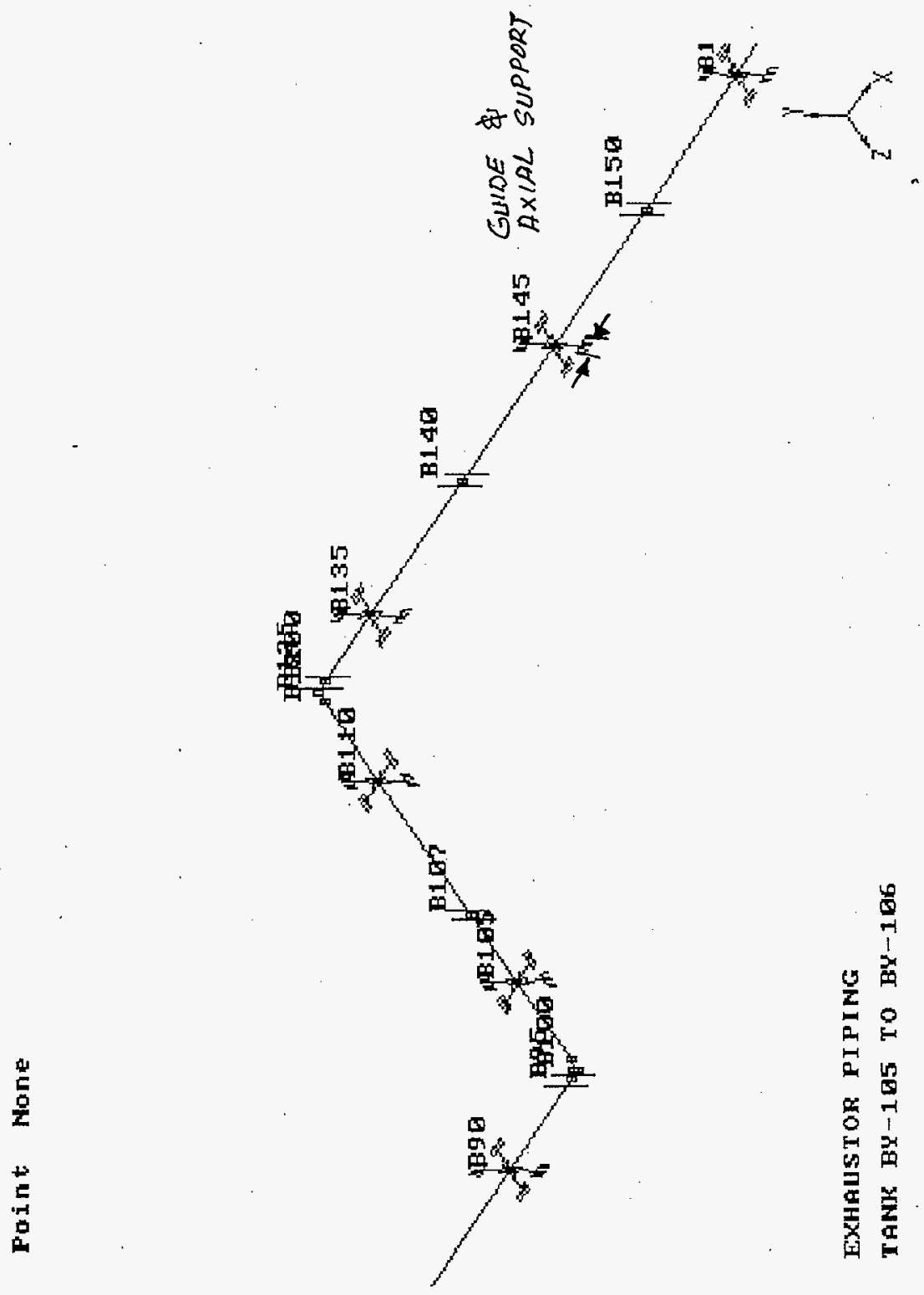



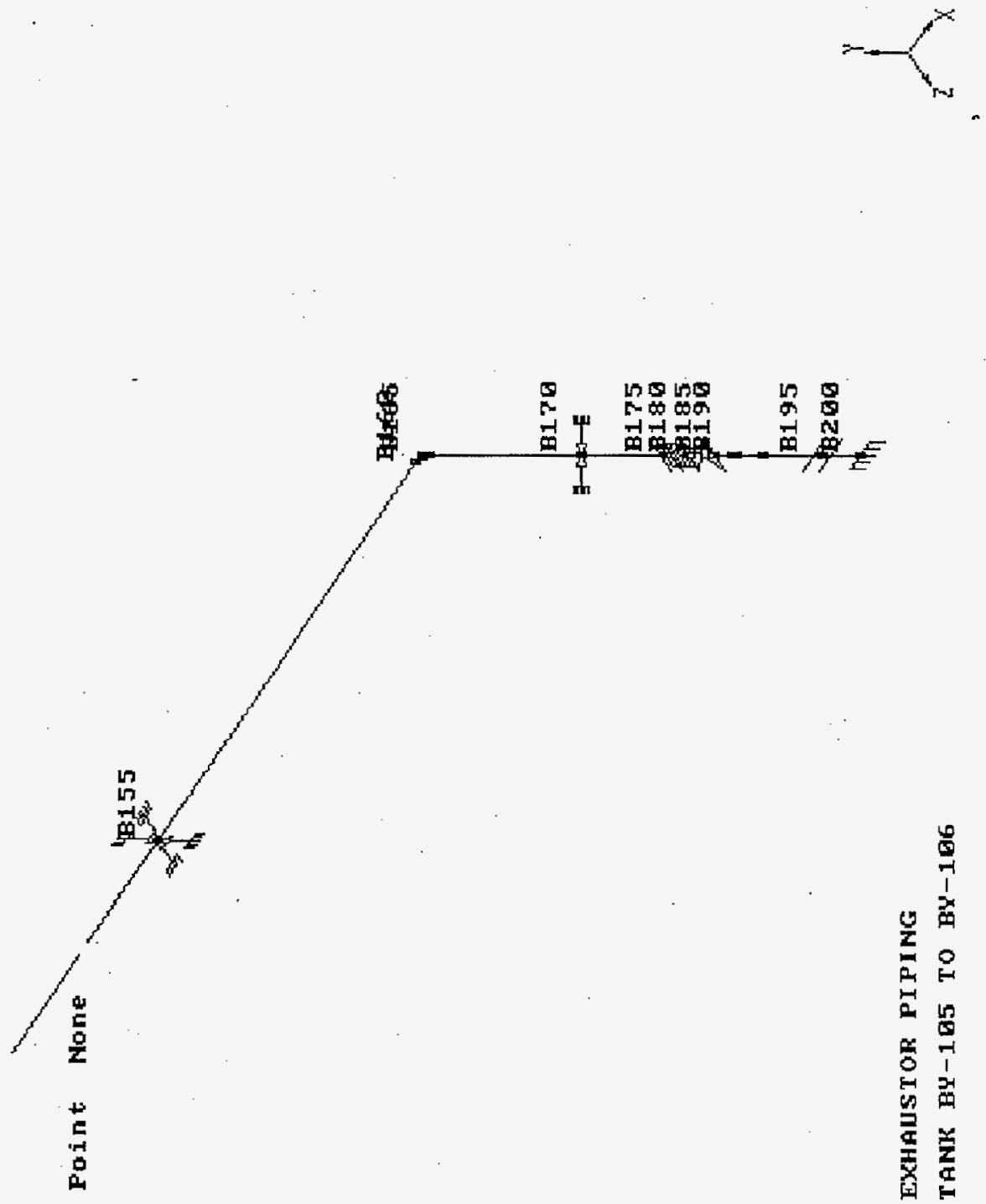
HNF-SD-WM-DA-236

Rev. 0

APPENDIX D: DESIGN CALCULATIONS FOR TANK S-107 INSTALLATION 
Fluor Daniel Northwest, Inc.

CALCULATION IDENTIFICATION AND INDEX
Page 1 of

Date

April 17, 1997

This sheet shows the status and description of the attached Design Analysis sheets.

Discipline Mechanical

wo/sob No. E40690

Calculation No. M-4

Project No. \& Name S-107 1000 CFM Exhauster Installation

Calculation Item

These calculations apply to:

Dwig. Ho. ECN 618794

Rev. No.

Dwg. No.

Rev. No.

other (Study, CDR)

Rev. No.

The status of these calculations is:

[] Preliminary Calculations

[X] Final Calculations

[] Check Calculations (On Calculation Dated)

[] Void Calculation (Reason Voided )

Incorporated in Final Drawings?

This calculation verified by independent "check" calculations?

[] Yes [X] No

[] Yes [] No

Original and Revised Calculation Approvals:

\begin{tabular}{|c|c|c|c|}
\hline & $\begin{array}{c}\text { Rev. } 0 \\
\text { Signature/Date }\end{array}$ & $\begin{array}{c}\text { Rev. } 1 \\
\text { Signature/Date }\end{array}$ & $\begin{array}{c}\text { Rev, } 2 \\
\text { Signature/Date }\end{array}$ \\
\hline Originator & & & \\
\hline \multicolumn{4}{|l|}{ Checked by } \\
\hline \multicolumn{4}{|l|}{ Approved by } \\
\hline $\begin{array}{l}\text { Checked Against } \\
\text { Approved Vendor Data }\end{array}$ & & & \\
\hline
\end{tabular}

\begin{tabular}{|c|l|}
$\begin{array}{l}\text { Design Analysis } \\
\text { Page No. }\end{array}$ & INOEX \\
\hline 3 & Analysis of piping and supports \\
\hline 3 & Analysis of tank inlet HEPA filter installation \\
\hline 4 & Analysis of exhauster inlet \\
\hline 7 & Determination of ventilation pressure losses \\
\hline & \\
\hline 10 & Attachments \\
\hline & Autopipe piping analysis results \\
\hline & \\
\hline & \\
\hline
\end{tabular}


Fluor Daniel Northwest, Inc.

\section{DESIGN ANALYSIS}

client LMHC

subject S-107 Exhauster Installation

Location $241-S-107$ calc. No. $M-4$

Revision 0

Page No. 2 of 9

wo/Job No. E40690

Date $4 /-17-9>$

checked $5728 / 97$

Revised
By C. M. Keller

By D.A. MOODK

By

\section{OBJECTIVE:}

Perform analysis of exhauster and HEPA filter installation on waste tank 241-S107 to verify stresses are within acceptable values and to determine expected pressure drop in the ventilation ducting.

\section{CRITERIA:}

Ventilation and condensate piping are considered safety significant/safety class 2. The waste tank including risers are considered safety class 1 . Design loads for analysis are specified in Design Loads for Facilities GC-LOAD-01, Rev 1. Piping is analyzed using criteria specified in the ASME B31.3 Process Piping Code.

\section{ASSUMPTIONS/GIVENS:}

Piping stresses and support loads used in the analysis were determined using Autopipe and are provided in the Attachments section. Analys is results for comparable installation designs have been applied where possible. These other installations were analyzed in calculations M-1 through M-3 of the same Job Number.

\section{CALCULATION METHOD:} design.

Hand calculations and Autopipe analysis have been used to verify adequacy of the

\section{REFERENCES:}

1) Manual of Steel Construction, Allowable Stress Design, 9th edition, American Society of Civil Engineers.

2) Marks Standard Handbook for Mechanical Engineers, 9th edition, McGraw-Hill 


\section{HNF-SD-WM-DA-236, Rev 0}

KAISER ENGINEERS HANFORD

Client

Subject
DESIGN ANALYSIS
Page D-5

Calc. No. $M-4$

Revision 0

Page No.

Wo/Job No. E 40690

Date $4 / 1>14 ?$ By CN KELLER
Checked 5/28/9? By DA NaOry Revised

3) REACTIONS ON THE CONDENSOR PIT CONERAND: EXHAUSTER PLENUM: WERE DETERMINED IN THE ATTACHEO AUTOPIPE ANALYSIS. REACTIONS ARE LESS THEN; OR ONLY SLIGATLY GREATER, THEN ANALYZED IN CALCULATION M-3. SATISFACTORY RESULTS, WITH SIGNIFICANT SAFET' MARGIN, IN THEE PREVIOUS ANACYSIS IS ATPLICABLE TO THIS INSTPOLLATON:

4) THE TANK INLET HEPA FITERE INSTALLATION DESIGN IS THE SAME AS THAT WHKH WAS ANALYZED IN. CALCULATION M-I. ANALYSIS OF THE FILTER INSTALLATION DESIGMI PROVIDED SATISFACTORY RESULTS. 
KAISER ENGINEERS

HNF-SD-WM-DA-236, Rev 0

Calc. No. Page D-6,

DESIGN ANALYSIS

Revision 0

Paps No. $\frac{5}{5}$ of 9

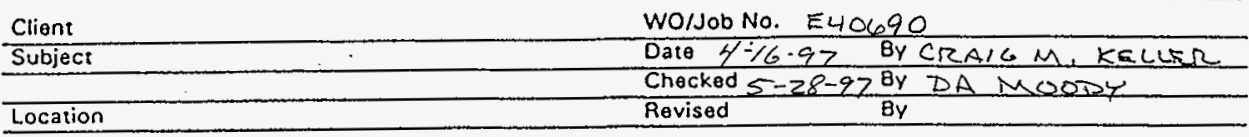

5) REAGTIONS ON EXHAHSTER MLET:

$$
\begin{array}{ll}
F_{x}=31 * & M_{x}=308 \mathrm{fH} / \mathrm{b} \\
F_{y}=320 * & M_{y}=36 \mathrm{fH} / \mathrm{b} \\
F_{z}=29 * & M_{z}=70 \mathrm{fW} / 1 \mathrm{~b}
\end{array}
$$

REACTIONS ON INLET SUPPORT ARE AS FOLLOWS:
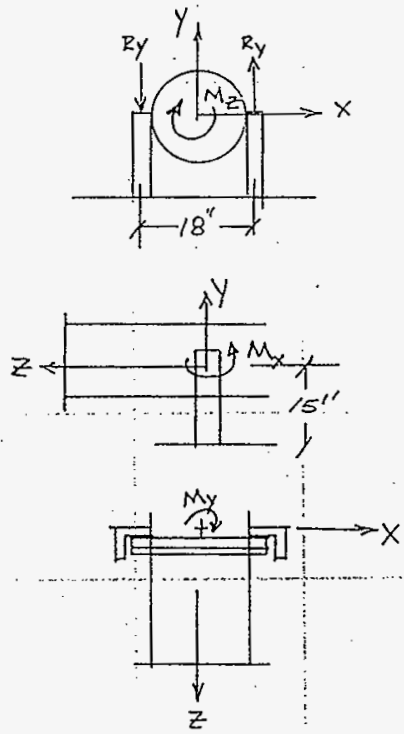

REACTION DUE TO $M_{Z}$ :

$$
\begin{aligned}
& M_{z}=2 R_{y}(9 / 12) \\
& 70=2\left(R_{y}\right)(9 / 12) \Rightarrow R_{y}=47^{*}
\end{aligned}
$$

REACTION DUE TO $M_{X}$ :

$$
\begin{aligned}
& M_{x}=2 R_{z}\left(15 / 1^{\prime}\right) \\
& 308=2\left(R_{z}\right)\left(15 / 12^{\prime}\right) \Rightarrow R_{z}=123^{*}
\end{aligned}
$$

Reaction DuE to My:

$$
\begin{aligned}
M_{y} & =2 R_{z}\left(9 / 1 z^{\prime}\right) \\
36 & =2\left(R_{z}\right)\left(9 / 1 z^{\prime}\right) \Rightarrow R_{z}=24^{*}
\end{aligned}
$$

TOTaL SUPPORT FORCES:

$$
\begin{aligned}
& F_{x}=31^{\not t} \\
& F_{y}=320+47=367^{* 2} \\
& F_{z}=29+123+24=176^{* 7}
\end{aligned}
$$

54.4300 .037 KEH.0037.00 (06/92) 


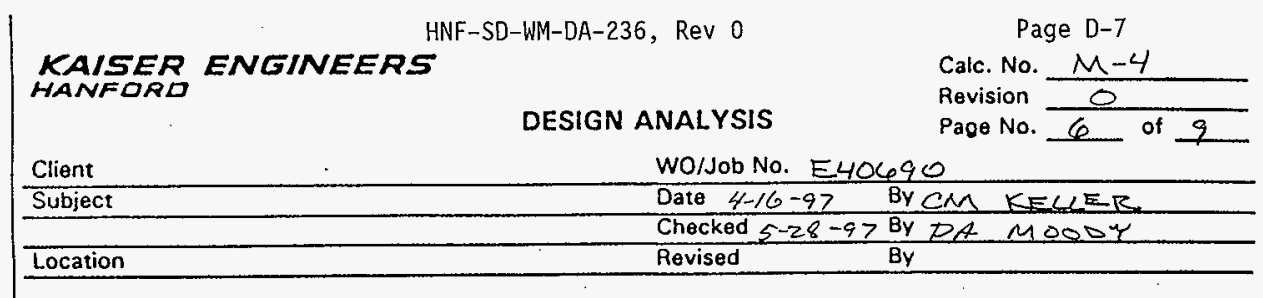

For Combined Compression and Bending strEsses,

$$
\frac{\Delta_{a}}{s_{a}}+\frac{\Delta_{b x}}{s_{b x}}+\frac{\Delta_{b z}}{s_{b z}} \leq 1 \text { rf } 1 \text { eq } H /-3
$$

where, $\Delta_{a}=$ AXIAL COMPRESSIVE stress

$\Delta_{b x}=$ Bending Stress in $x$-dir

$\Delta_{b e}=$ Bending Stress in $z$-dir

$S_{a}, S_{b_{X}}, S_{b_{z}}=$ AlLOWABLE STRESS $=22 \mathrm{ks}$.

$$
\begin{aligned}
& \Delta_{A}=\frac{F_{y}}{A}=\frac{367^{\text {th }}}{1.19 \mathrm{im}^{2}}=308 \mathrm{psi} \\
& \Delta_{b x}=\frac{F_{x}\left(15^{\prime \prime}\right)}{S_{x x}}=\frac{31(15)}{.260 \mathrm{in}^{3}}=1788 \mathrm{psi} \\
& \Delta_{b_{z}}=\frac{F_{z}\left(15^{\prime \prime}\right)}{S_{z z}}=\frac{176(15)}{.542 \mathrm{in}^{3}}=487 \mathrm{psi}
\end{aligned}
$$

$$
\frac{308}{22000}+\frac{1788}{22000}+\frac{4871}{22000}=.32<1<K
$$

$54-4300-037 \mathrm{KEH}-0037.00(06 / 92)$ 


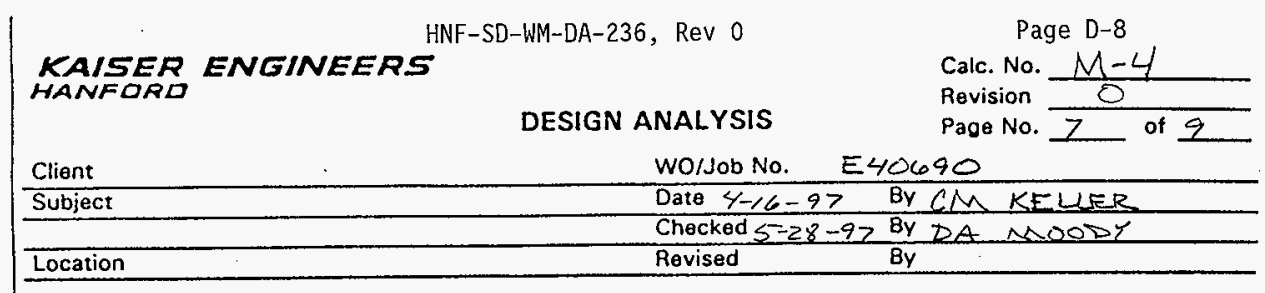

CALCULATE PRESSURE DROP FROM ATMOSPHERE . TOEXHAUSTER INLET.

saturated 4 ir (9) $140^{\circ} \mathrm{F}$

FLOWRATE $Q=500 d F M$ (LOWER RANGE OF EXHAMSTER)

ASSUME I" WIG, Pressure drop FOR INLET FILTER

DeTermine $\triangle P$ LOSS FOR THE FOLLOWING:

- tank inlet filter and riser

- TANK OUTLET

- VENTILATION PLENUM

TANK INLET FILTER AND RISER:

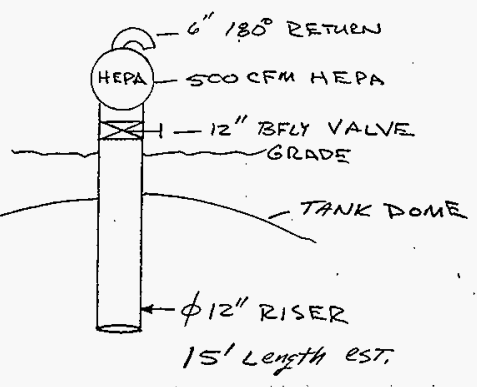

LOSSES

$6^{\prime \prime} 180^{\circ}$ RETURN $\angle \mathrm{e}=25^{\prime}$ of 2 HERA FILTER $\triangle P=1$ "WG eAt. BFLY VALVE Le $=23^{\prime} \mathrm{es}$. $12^{\prime \prime} R$ SER $L=15^{\prime}$ ref? INLET LOSS Le $=73^{\prime}$ ref 2

$54-4300.037$ KEH.0037.00 (06/92) 


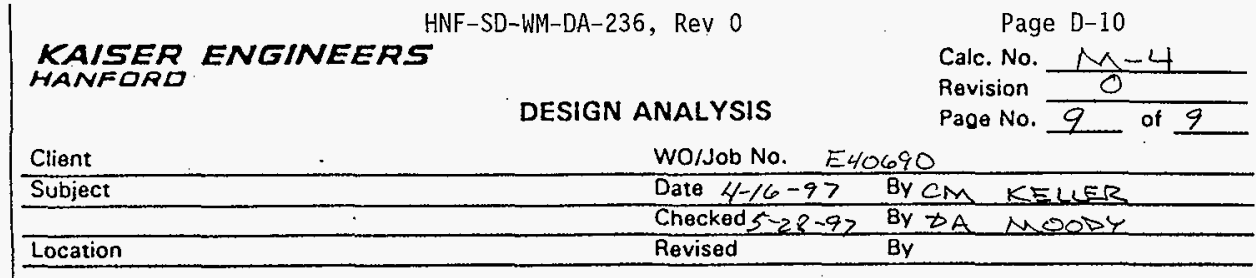

VENTILATION LOSSES FOR SOOCFM IN G" AND IL" DUCT DIAMETERS WERE DETERMINED IN CALCULATION MI

$c^{\prime \prime} \Delta_{p}=1.19$ in $w / 100^{\prime}$

$12^{\prime \prime} \quad \Delta p=.04$ in $2 y / 100^{\prime}$

TANK INLET COSS:

$$
\begin{gathered}
\Delta P_{\text {MEET }}=25^{\prime}(1119 \text { in wy /100 })+1 " \text { "Wy }+(23+15+73)\left(.04 \frac{\text { in wy }}{100^{\prime}}\right) \\
\Delta P_{\text {INLET }}=1.34 \text { in wy }
\end{gathered}
$$

VENTILATION PLENUM LOSS:

$$
\begin{aligned}
& \Delta p=(19+10+23+27+10 \times 13+22)\left(1.19 \frac{\text { in wy }}{100}\right)+\left(20^{\prime}\right)\left(.04 \frac{\text { in wy }}{100^{\prime}}\right) \\
& \Delta p_{\text {PLENum }}=1.48 \mathrm{incyg} \\
& \text { Total } \cos 5=2.82 \text { in mg } 0 K
\end{aligned}
$$

$54-4300.037 \quad \mathrm{KEH}-0037.00(06 / 92)$ 


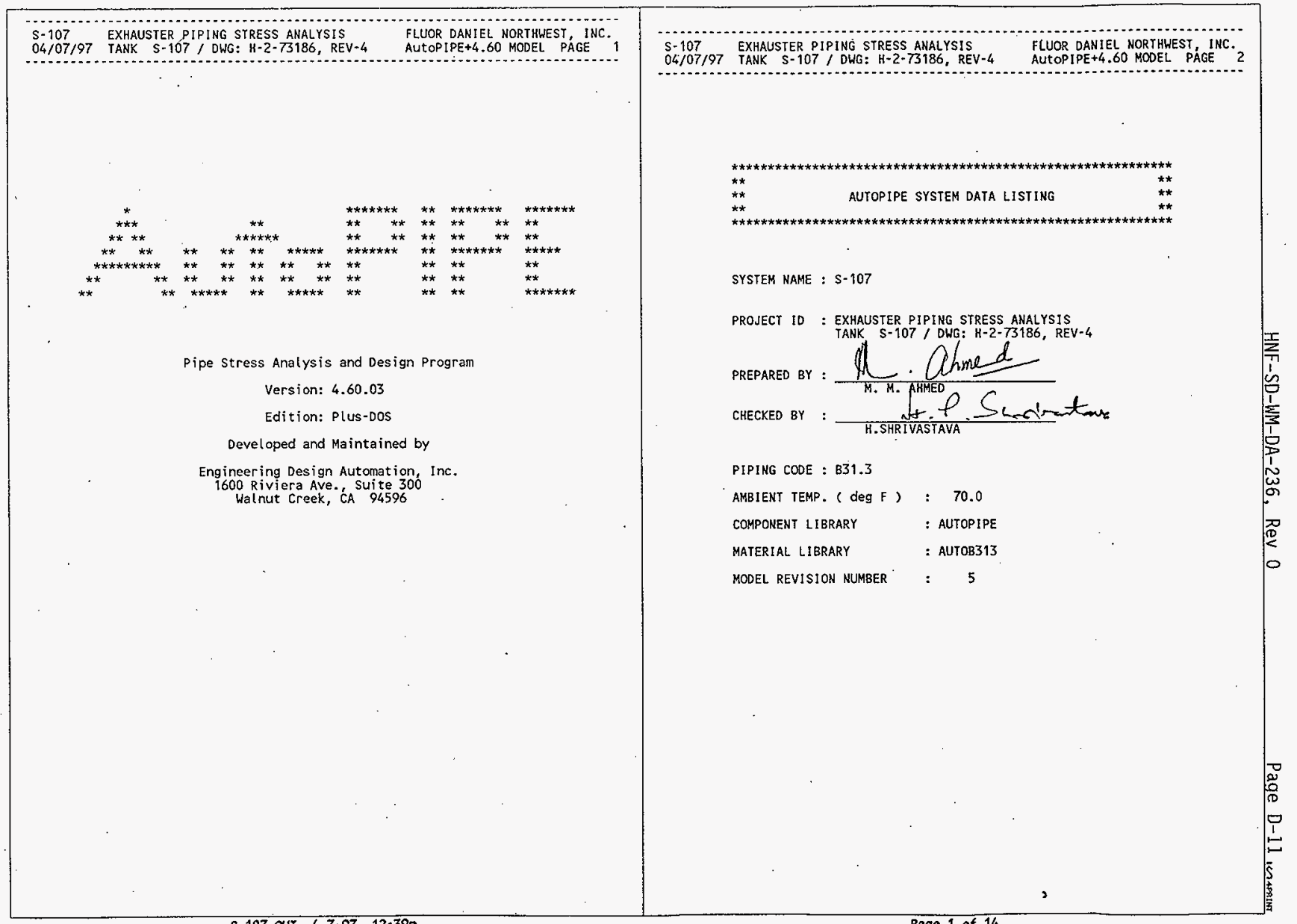




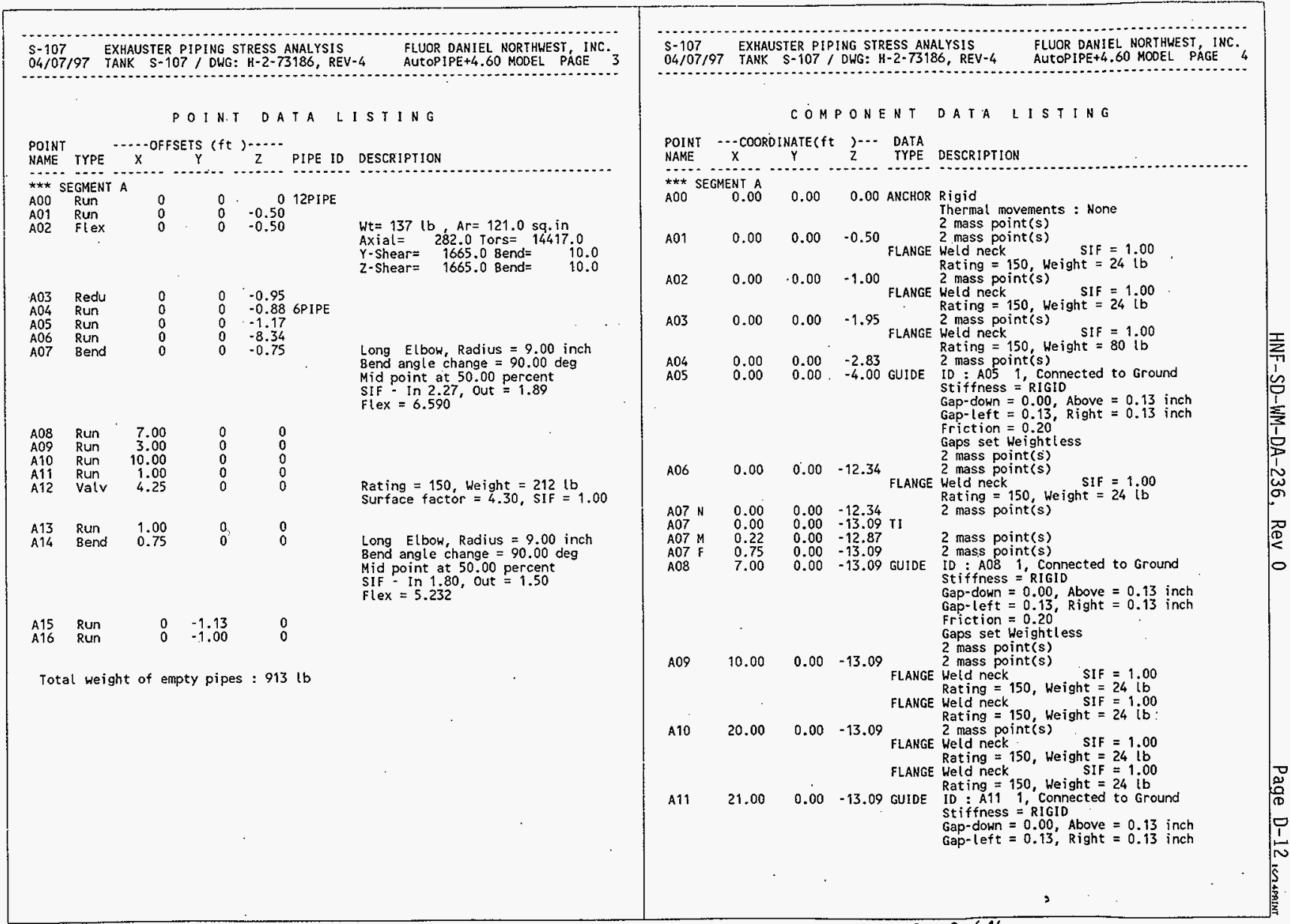




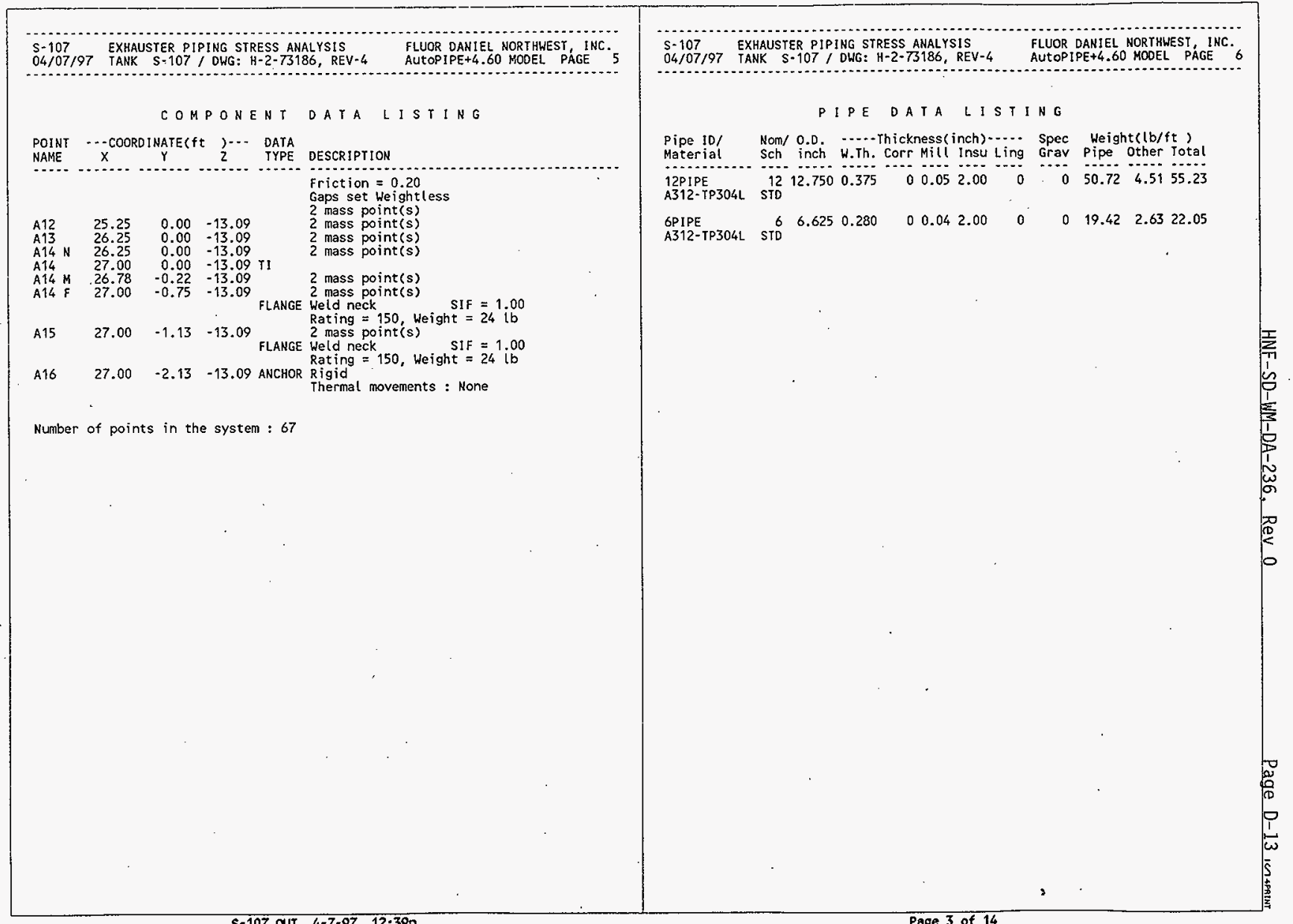




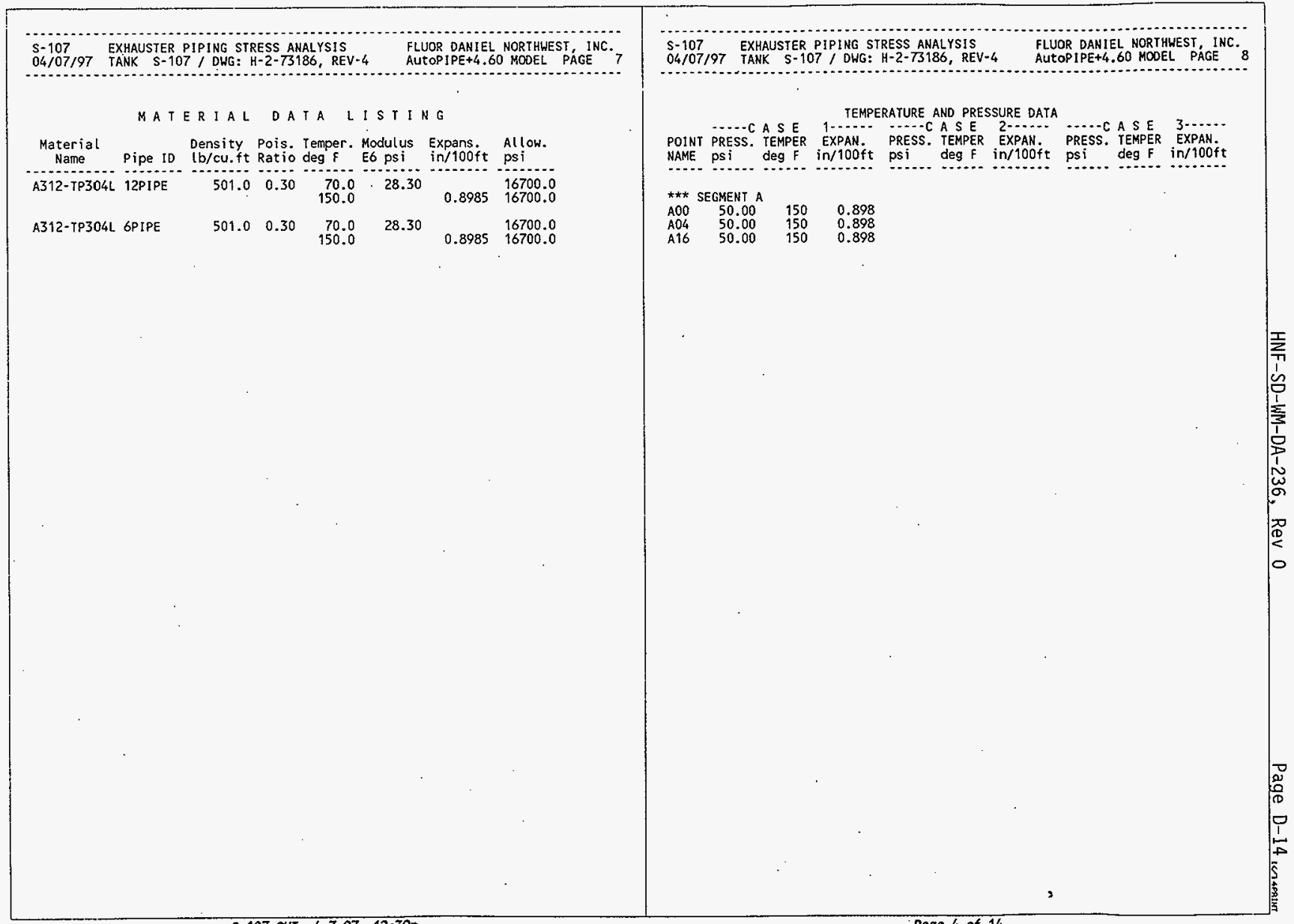


S-107 EXHAUSTER PIPING STRESS ANALYSIS FLUOR OANIEL NORTHWEST, INC. 04/07/97 TANK S-107/ DWG: H-2-73186, REV-4 AUTOPIPE+4.60 RESULT PAGE 1

ANALYSIS SUMMARY

Current model revision number : 5

Static - Date and Time of analys is Model Revision Number

Apr 7, $1997 \quad 11: 04$ AM Number of load cases

5

oad cases analyzed............. GR II

Gaps/Friction/Yielding considered ..... Yes

Tolerance - Force, Displacement ........ $100.00 \mathrm{lb}, 0.0162$ in

Friction - Factor tolerance........ $.1 .00 \quad 0.10$

Hanger design run...$\ldots \ldots \ldots \ldots \ldots \ldots \ldots$. No

Cut short included ................... No

Occasional load analysis type $\ldots \ldots \ldots$ Nonl inear

Non-linear analys is sumary file ...... s-107.LOG

Base load cases for nonlinear analys is

$$
\begin{aligned}
& \mathrm{GR}=\text { None } \\
& \mathrm{T} 1=\mathrm{GR}
\end{aligned}
$$

Weight of contents included.... Yes

Number of mass points per span ..........

Pressure stiffening case ...............

Water elevation for buoyancy ioads ...... Not considered

Modal - Date and time of analysis .......... Apr 7, 1997 11:05 AM Model Revision Number ............... 5

Number of modes $\ldots \ldots \ldots \ldots \ldots \ldots \ldots \ldots, 18$

Cutoff frequency $(\mathrm{Hz}) \ldots \ldots \ldots \ldots \ldots \ldots$.

Weight of contents included $\ldots \ldots \ldots \ldots$. Y

Pressure stiffening case .................

Water el evation for buoyancy loads ..... Not considered

Response - Date and Time of analysis ............ Apr 7, 1997 11:05 AM Model Revision Number .................. 5

Number of load cases $\ldots \ldots \ldots \ldots \ldots \ldots \ldots \ldots 1$

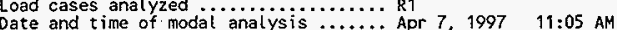

Date and time of modal analys is $\ldots \ldots \ldots$ Ap

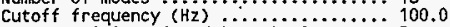

Model revision of modal analysis ...... 5

Weight of contents included ........... Yes

Number of mass points per span ........ 2

Water elevation for buoyancy loads .... Not considered

$\begin{array}{lll}\text { S }-107 & \text { EXHAUSTER PIPING STRESS ANALYSIS } & \text { FLUOR DANIEL NORTHWEST, INC. } \\ 04 / 07 / 97 & \text { TANK } 5-107 & \text { AUTOPIPE+4.60 RESULT PAGE }\end{array}$

04/07/97 TANK S-107 / OWG: H-2-73186, REV-4

UT OPIPE+4.60 RESULT PAGE

COOE COMPLIANCE COMBINATIONS

Combination Category Method Load Factor Allowable Remarks GR + Maxp sustain Sum Gravity 1.00 Automatic Default cold to T1 Expansion Sum Thermal 11.00 Automatic Defaul Sus. + R1 Occasion Abs sum Response 11.00 Automatic Default Max P Hoop Max Hoop 1.00 Automatic Default

\section{OTHER USER COMBINATIONS}

\begin{tabular}{|c|c|c|c|c|}
\hline $\begin{array}{l}\text { Combination } \\
\text { GR }\end{array}$ & $\begin{array}{l}\text { Method } \\
\text { Sun }\end{array}$ & $\begin{array}{l}\text { Load } \\
\text { Gravity }\end{array}$ & \begin{tabular}{c} 
Factor \\
\hdashline 1.00
\end{tabular} & $\begin{array}{l}\text { Remarks } \\
\text { Default }\end{array}$ \\
\hline T1 & sum & Thermal 1 & 1.00 & Default \\
\hline R1 & Sum & Response 1 & 1.00 & Defaut \\
\hline $\mathrm{GR}+\mathrm{T} 1$ & Sum & $\begin{array}{l}\text { Gravity } \\
\text { Thermal } 1\end{array}$ & $\begin{array}{l}1.00 \\
1.00\end{array}$ & User \\
\hline$G R+T 1+S E$ IS & Abs sum & $\begin{array}{l}\mathrm{GR}+\mathrm{T} 1 \\
\text { Response } 1\end{array}$ & $\begin{array}{l}1.00 \\
1.00\end{array}$ & User \\
\hline TOTAL. & Abs sum & $\begin{array}{l}\mathrm{GR}+\mathrm{T1} \\
\text { Response } 1\end{array}$ & $\begin{array}{l}1.00 \\
1.00\end{array}$ & User \\
\hline
\end{tabular}

CODE COMPLIANCE

Y - Factor ...................... 0.40 Weld efficiency factor $\ldots \ldots \ldots \ldots \ldots \ldots \ldots .1 .00$

Range reduction factor ................ 1.00

Design Pressure Factor .............. 1.00

Minimum stress ratio used in reports... 0.00

Include corrosion in stress calcs. .... Y

Include torsion in code stress ..........

Include axial force in code stress ..... N

Longitudinal pressure calculation ...... PD/4t

Include rigorous pressure ............. Not analyzed 


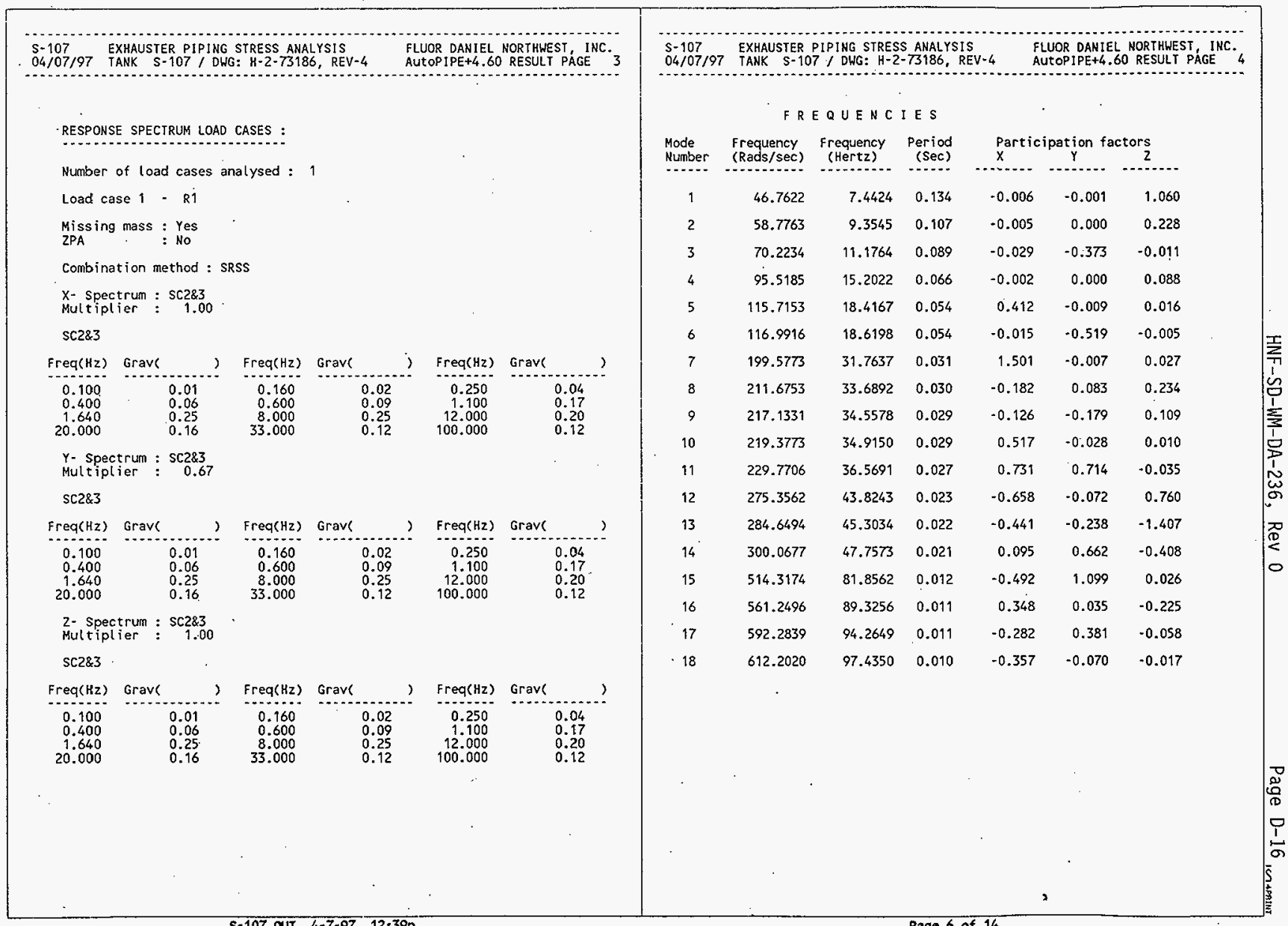




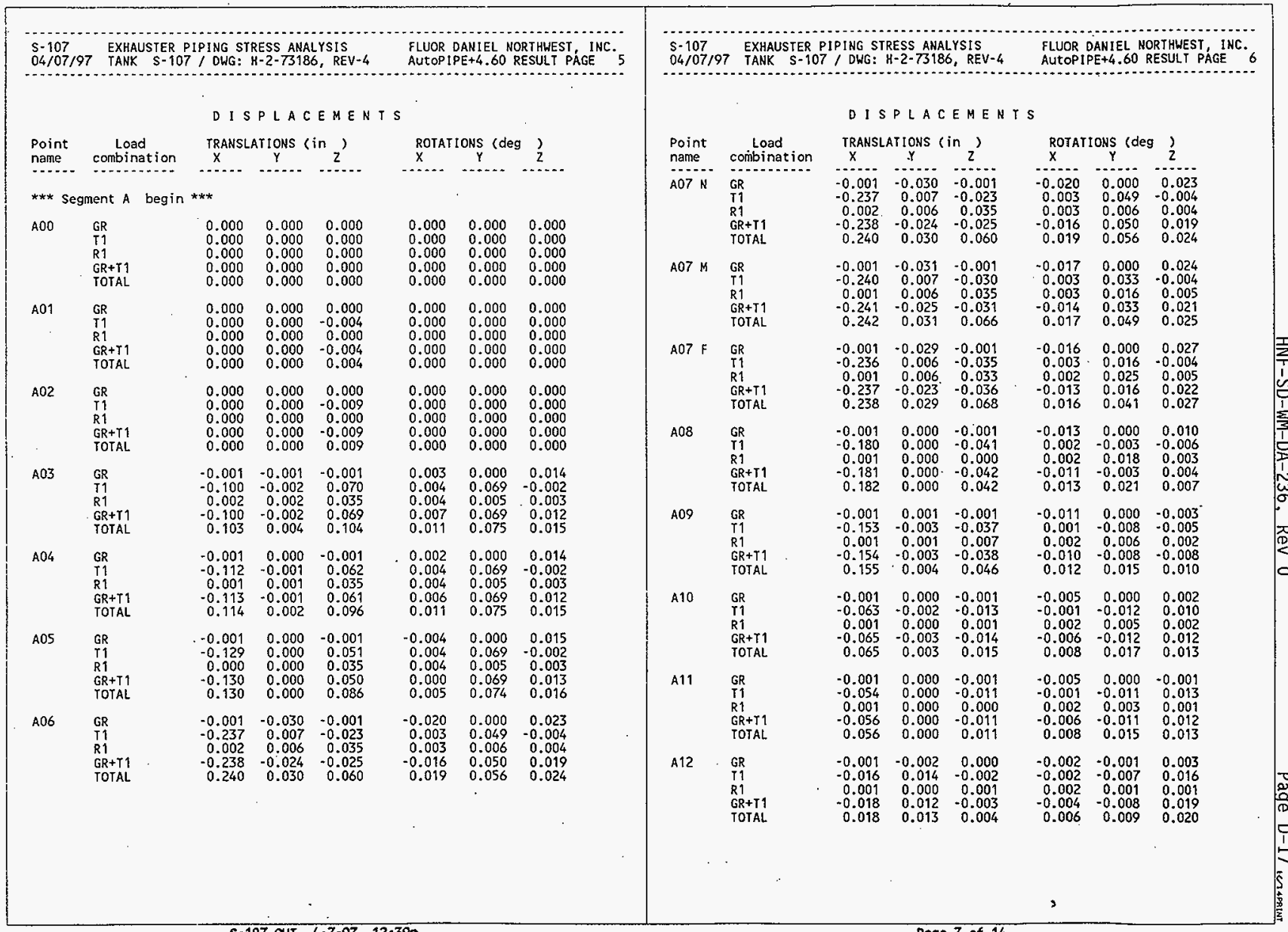




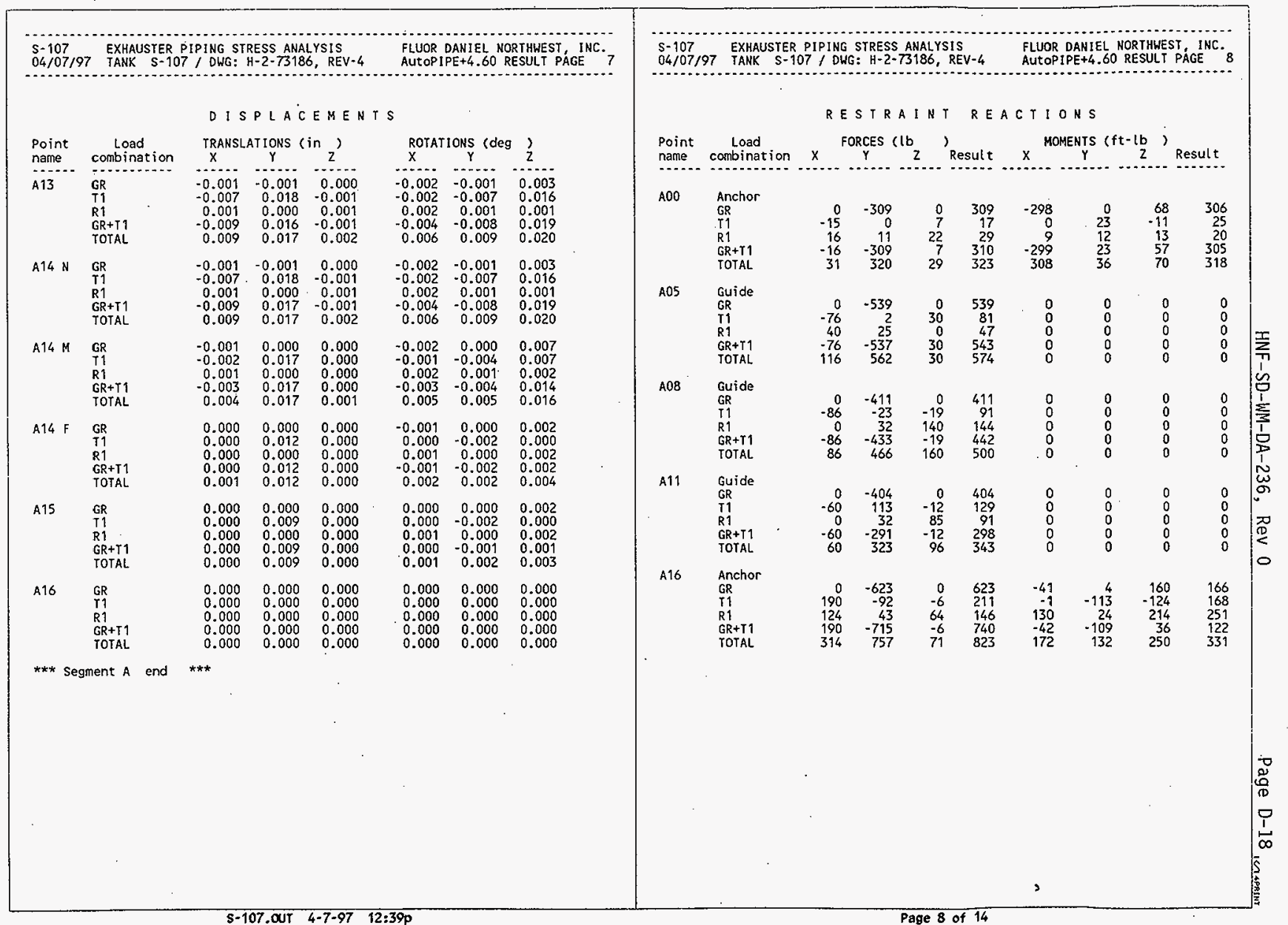




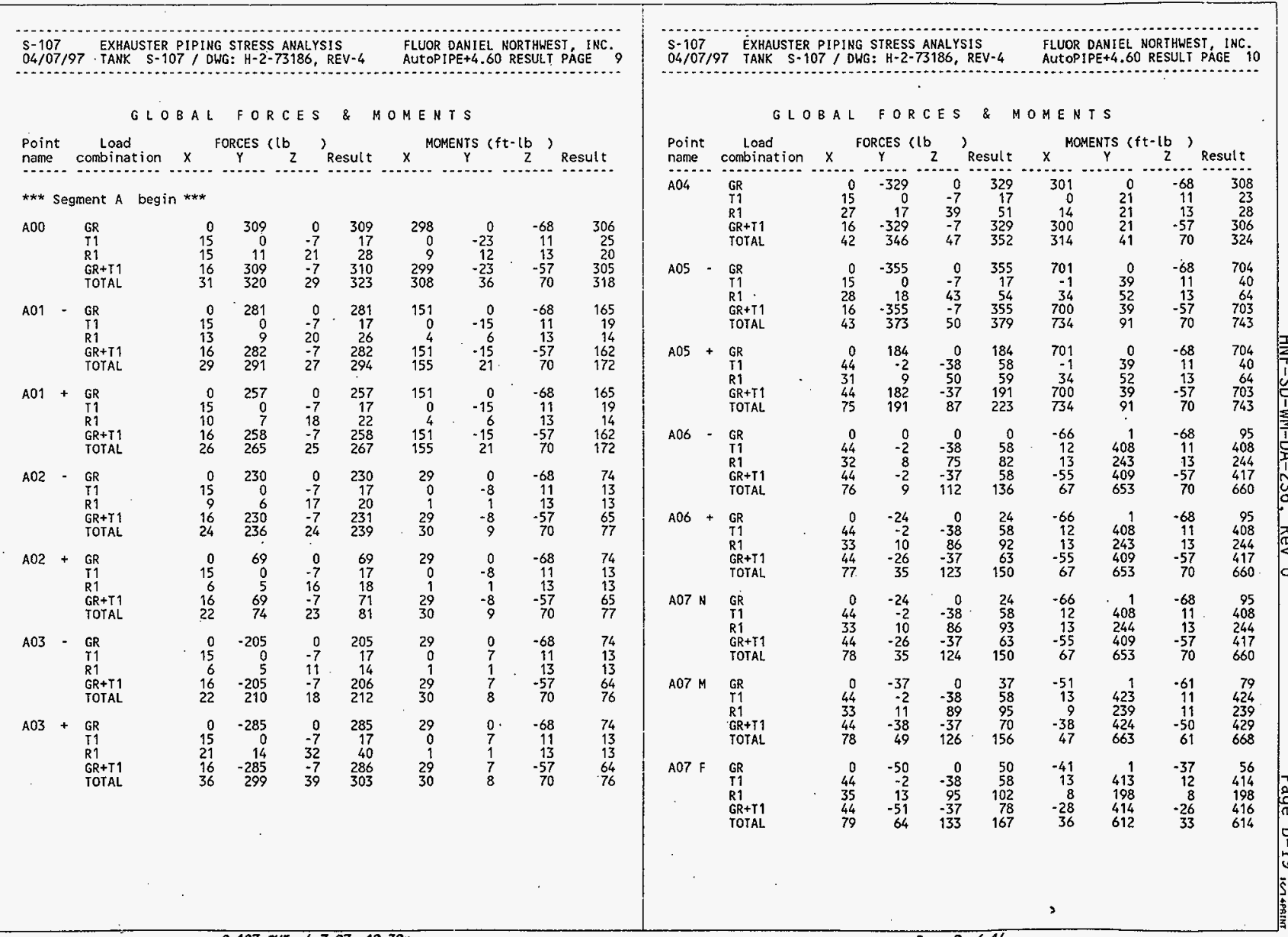




\begin{tabular}{|c|c|c|c|c|c|c|c|c|c|c|c|c|c|c|c|c|c|c|c|c|}
\hline \multirow{3}{*}{\multicolumn{2}{|c|}{$\begin{array}{l}5-107 \\
04 / 07 / 9 \\
- \\
\begin{array}{l}\text { Point } \\
\text { name }\end{array}\end{array}$}} & \multicolumn{5}{|c|}{$\begin{array}{l}\text { EXHAUSTER PIPING STRESS ANALYSIS } \\
97 \text { IANK S-107/ OWG: H-2-73186, REV-4 }\end{array}$} & \multicolumn{4}{|c|}{$\begin{array}{l}\text { FLUOR OANIEL NORTHWEST INC } \\
\text { AUTOPIPE+4.60 RESULT PAGE i1 }\end{array}$} & \multicolumn{6}{|c|}{$\begin{array}{ll}\text { S-107 } & \text { EXHAUSTER PIPING STRESS ANALYSIS } \\
04 / 07 / 97 & \text { TANK S-107/ DWG: } H-2-73186, \text { REV-4 }\end{array}$} & \multicolumn{4}{|c|}{$\begin{array}{l}\text { FLUOR DANIEL NORTHWEST, INC, } \\
\text { AUTOPIPE+4.60 RESULT PAGE I2 }\end{array}$} \\
\hline & & \multicolumn{9}{|c|}{ GLOBAL FORCES \& MOMENTS } & \multicolumn{10}{|c|}{ GLOBAL FORCES \& MOMENTS } \\
\hline & & $\begin{array}{l}\text { Load } \\
\text { combination }\end{array}$ & $x$ & RCES ( & $b_{Z} \quad{ }_{R}$ & Result & $x^{\text {MOI }}$ & ITS $(f$ & $\left.\begin{array}{rl}-1 b \\
2\end{array}\right)$ & Result & $\begin{array}{l}\text { Point } \\
\text { name }\end{array}$ & $\begin{array}{l}\text { Load } \\
\text { combination }\end{array}$ & $x$ & ORCES & z & Result & $x^{\text {MON }}$ & $\underset{Y}{\text { ENTS }}<\mathrm{ft}$ & $\left.-\begin{array}{rl}-1 b \\
Z\end{array}\right)_{R}$ & Result \\
\hline$A 08$ & - & $\begin{array}{l}G R \\
T 1 \\
R 1 \\
\text { GR+TI } \\
\text { TOTAL }\end{array}$ & $\begin{array}{l}0 \\
44 \\
38 \\
44 \\
83\end{array}$ & $\begin{array}{r}-188 \\
-2 \\
15 \\
-189 \\
205\end{array}$ & $\begin{array}{r}0 \\
-38 \\
103 \\
-37 \\
141\end{array}$ & $\begin{array}{r}188 \\
58 \\
111 \\
198 \\
262\end{array}$ & $\begin{array}{r}-41 \\
13 \\
8 \\
-28 \\
36\end{array}$ & $\begin{array}{l}179 \\
425 \\
181 \\
605\end{array}$ & $\begin{array}{r}705 \\
21 \\
80 \\
727 \\
807\end{array}$ & $\begin{array}{r}707 \\
180 \\
432 \\
749 \\
1010\end{array}$ & A12 & $\begin{array}{l}\text { GR } \\
\text { T1 } \\
\text { R1 } \\
\text { GR+T1 } \\
\text { TOTAL }\end{array}$ & $\begin{array}{r}0 \\
190 \\
80 \\
190 \\
270\end{array}$ & $\begin{array}{r}129 \\
-92 \\
13 \\
37 \\
50\end{array}$ & $\begin{array}{l}0 \\
-6 \\
31 \\
-6 \\
38\end{array}$ & $\begin{array}{r}129 \\
211 \\
87 \\
194 \\
277\end{array}$ & $\begin{array}{r}-41 \\
13 \\
8 \\
-28 \\
36\end{array}$ & $\begin{array}{r}4 \\
-102 \\
57 \\
-97 \\
155\end{array}$ & $\begin{array}{r}-430 \\
119 \\
69 \\
-311 \\
372\end{array}$ & $\begin{array}{r}432 \\
157 \\
84 \\
327 \\
404\end{array}$ \\
\hline A08 & + & $\begin{array}{l}G R \\
I 1 \\
R 1 \\
G R+r 1 \\
\text { IOTAL }\end{array}$ & $\begin{array}{r}0 \\
130 \\
40 \\
130 \\
170\end{array}$ & $\begin{array}{r}223 \\
21 \\
21 \\
244 \\
265\end{array}$ & $\begin{array}{r}0 \\
-18 \\
39 \\
-18 \\
57\end{array}$ & $\begin{array}{r}223 \\
133 \\
60 \\
277 \\
320\end{array}$ & $\begin{array}{r}-41 \\
13 \\
8 \\
-28 \\
36\end{array}$ & $\begin{array}{l}179 \\
425 \\
181 \\
605\end{array}$ & $\begin{array}{r}705 \\
21 \\
80 \\
727 \\
807\end{array}$ & $\begin{array}{r}707 \\
180 \\
432 \\
749 \\
1010\end{array}$ & A 13 & $\begin{array}{l}\text { GR } \\
\text { I1 } \\
R 1 \\
\text { GR+Y1 } \\
\text { TOTAL }\end{array}$ & $\begin{array}{l}10 \\
190 \\
120 \\
190 \\
310 .\end{array}$ & $\begin{array}{r}-518 \\
-92 \\
39 \\
-610 \\
649\end{array}$ & $\begin{array}{l}0 \\
-6 \\
60 \\
-6 \\
67\end{array}$ & $\begin{array}{l}518 \\
211 \\
140 \\
639 \\
722\end{array}$ & $\begin{array}{r}-41 \\
13 \\
8 \\
-28 \\
36\end{array}$ & $\begin{array}{r}4 \\
-108 \\
29 \\
-104 \\
133\end{array}$ & $\begin{array}{r}-236 \\
212 \\
54 \\
-24 \\
79\end{array}$ & $\begin{array}{r}239 \\
238 \\
62 \\
110 \\
159\end{array}$ \\
\hline$A 09$ & - & $\begin{array}{l}\text { GR } \\
T 1 \\
R 1 \\
\text { GR+TI } \\
\text { TOTAL }\end{array}$ & $\begin{array}{r}0 \\
130 \\
43 \\
130 \\
173\end{array}$ & $\begin{array}{r}157 \\
21 \\
19 \\
178 \\
197\end{array}$ & $\begin{array}{r}0 \\
-18 \\
39 \\
-18 \\
56\end{array}$ & $\begin{array}{r}157 \\
133 \\
60 \\
221 \\
268\end{array}$ & $\begin{array}{r}-41 \\
13 \\
8 \\
-28 \\
36\end{array}$ & $\begin{array}{l}124 \\
317 \\
127 \\
444\end{array}$ & $\begin{array}{r}135 \\
-42 \\
58 \\
94 \\
151\end{array}$ & $\begin{array}{l}141 \\
132 \\
323 \\
160 \\
471\end{array}$ & $\mathrm{~A} 14 \mathrm{~N}$ & $\begin{array}{l}\text { GR } \\
\text { I1 } \\
R 1 \\
\text { GR+T1 } \\
\text { TOTAL }\end{array}$ & $\begin{array}{l}0 \\
190 \\
120 \\
190 \\
310\end{array}$ & $\begin{array}{r}-518 \\
-92 \\
39 \\
-610 \\
649\end{array}$ & $\begin{array}{l}0 \\
-6 \\
61 \\
-6 \\
67\end{array}$ & $\begin{array}{l}518 \\
211 \\
140 \\
639 \\
723\end{array}$ & $\begin{array}{r}-41 \\
13 \\
8 \\
-28 \\
36\end{array}$ & $\begin{array}{r}4 \\
-108 \\
29 \\
-104 \\
133\end{array}$ & $\begin{array}{r}-235 \\
212 \\
54 \\
-23 \\
77\end{array}$ & $\begin{array}{r}238 \\
238 \\
62 \\
110 \\
158\end{array}$ \\
\hline$A 09$ & + & $\begin{array}{l}\text { GR } \\
\text { II } \\
R 1 \\
\text { GR+T1 } \\
\text { TOTAL }\end{array}$ & $\begin{array}{r}0 \\
130 \\
48 \\
130 \\
179\end{array}$ & $\begin{array}{r}109 \\
21 \\
11 \\
130 \\
141\end{array}$ & $\begin{array}{r}0 \\
-18 \\
41 \\
-18 \\
59\end{array}$ & $\begin{array}{r}109 \\
133 \\
64 \\
185 \\
235\end{array}$ & $\begin{array}{r}-41 \\
13 \\
8 \\
-28 \\
-36\end{array}$ & $\begin{array}{l}122 \\
317 \\
127 \\
444\end{array}$ & $\begin{array}{r}135 \\
-42 \\
58 \\
94 \\
151\end{array}$ & $\begin{array}{l}141 \\
132 \\
323 \\
160 \\
471\end{array}$ & A14 M & $\begin{array}{l}\text { GR } \\
\text { T1 } \\
\text { R1 } \\
\text { GR+I1 } \\
\text { TOTAL }\end{array}$ & $\begin{array}{r}0 \\
190 \\
121 \\
190 \\
311\end{array}$ & $\begin{array}{r}-531 \\
-92 \\
39 \\
-623 \\
663\end{array}$ & $\begin{array}{l}0 \\
-6 \\
62 \\
-6 \\
68\end{array}$ & $\begin{array}{l}531 \\
211 \\
142 \\
652 \\
735\end{array}$ & $\begin{array}{r}-41 \\
12 \\
11 \\
-30 \\
40\end{array}$ & $\begin{array}{r}4 \\
-111 \\
15 \\
-107 \\
122\end{array}$ & $\begin{array}{r}42 \\
219 \\
24 \\
261 \\
285\end{array}$ & $\begin{array}{r}59 \\
246 \\
30 \\
284 \\
313\end{array}$ \\
\hline A10 & $\cdot$ & $\begin{array}{l}G R \\
T 1 \\
81 \\
\text { GR+T1 } \\
\text { TOTAL }\end{array}$ & $\begin{array}{r}0 \\
130 \\
58 \\
130 \\
188\end{array}$ & $\begin{array}{r}-112 \\
21 \\
16 \\
-91 \\
107\end{array}$ & $\begin{array}{r}0 \\
-18 \\
47 \\
-18 \\
65\end{array}$ & $\begin{array}{r}112 \\
133 \\
77 \\
160 \\
226\end{array}$ & $\begin{array}{r}-41 \\
13 \\
8 \\
-28 \\
36\end{array}$ & $\begin{array}{r}3 \\
-56 \\
120 \\
-53 \\
172\end{array}$ & $\begin{array}{r}148 \\
-251 \\
29 \\
-104 \\
133\end{array}$ & $\begin{array}{l}153 \\
258 \\
124 \\
120 \\
221\end{array}$ & A14 F- & $\begin{array}{l}\text { GR } \\
\text { T1 } \\
\text { R1 } \\
\text { GR+I1 } \\
\text { TOTAL }\end{array}$ & $\begin{array}{r}0 \\
190 \\
122 \\
190 \\
312\end{array}$ & $\begin{array}{r}-544 \\
-92 \\
40 \\
-636 \\
676\end{array}$ & $\begin{array}{l}0 \\
-6 \\
62 \\
-6 \\
68\end{array}$ & $\begin{array}{l}544 \\
211 \\
142 \\
664 \\
748\end{array}$ & $\begin{array}{r}-41 \\
8 \\
42 \\
-33 \\
75\end{array}$ & $\begin{array}{r}4 \\
-113 \\
24 \\
-109 \\
132\end{array}$ & $\begin{array}{r}160 \\
138 \\
45 \\
299 \\
344\end{array}$ & $\begin{array}{r}166 \\
179 \\
66 \\
320 \\
376\end{array}$ \\
\hline $\mathrm{A} 10$ & + & $\begin{array}{l}\text { GR } \\
T 1 \\
R 1 \\
\text { GR+T1 } \\
\text { TOTAL }\end{array}$ & $\begin{array}{r}0 \\
130 \\
64 \\
130 \\
194\end{array}$ & $\begin{array}{r}-160 \\
21 \\
22 \\
+139 \\
160\end{array}$ & $\begin{array}{r}0 \\
-18 \\
49 \\
-18 \\
67\end{array}$ & $\begin{array}{r}160 \\
133 \\
83 \\
191 \\
261\end{array}$ & $\begin{array}{r}-41 \\
13 \\
8 \\
-28 \\
36\end{array}$ & $\begin{array}{r}3 \\
-56 \\
120 \\
-53 \\
172\end{array}$ & $\begin{array}{r}148 \\
-251 \\
-29 \\
-104 \\
133\end{array}$ & $\begin{array}{l}153 \\
258 \\
124 \\
120 \\
221\end{array}$ & $\mathrm{~A} 14 \mathrm{Ft}$ & $\begin{array}{l}\text { GR } \\
\text { I1 } \\
\text { R1 } \\
\text { GR+I1 } \\
\text { TOTAL }\end{array}$ & $\begin{array}{r}0 \\
190 \\
123 \\
190 \\
313\end{array}$ & $\begin{array}{r}-568 \\
-92 \\
41 \\
-660 \\
701\end{array}$ & $\begin{array}{l}0 \\
-6 \\
63 \\
-6 \\
69\end{array}$ & $\begin{array}{l}568 \\
211 \\
144 \\
687 \\
771\end{array}$ & $\begin{array}{r}-41 \\
8 \\
42 \\
-33 \\
75\end{array}$ & $\begin{array}{r}4 \\
-113 \\
24 \\
-109 \\
132\end{array}$ & $\begin{array}{r}160 \\
138 \\
45 \\
299 \\
344\end{array}$ & $\begin{array}{r}166 \\
179 \\
66 \\
320 \\
376\end{array}$ \\
\hline A11 & - & $\begin{array}{l}\text { GR } \\
\text { II } \\
R 1 \\
\text { GR+TI } \\
\text { TOTAL. }\end{array}$ & $\begin{array}{r}0 \\
130 \\
65 \\
130 \\
195\end{array}$ & $\begin{array}{r}-182 \\
21 \\
23 \\
-161 \\
183\end{array}$ & $\begin{array}{r}0 \\
-18 \\
49 \\
-18 \\
67\end{array}$ & $\begin{array}{r}182 \\
133 \\
85 \\
208 \\
276\end{array}$ & $\begin{array}{r}-41 \\
13 \\
8 \\
-28 \\
36\end{array}$ & $\begin{array}{r}4 \\
-74 \\
167 \\
-70 \\
238\end{array}$ & $\begin{array}{r}318 \\
-272 \\
46 \\
46 \\
92\end{array}$ & $\begin{array}{r}321 \\
283 \\
174 \\
89 \\
258\end{array}$ & A15 & $\begin{array}{l}\text { GR } \\
\text { T1 } \\
\text { R1 } \\
\text { GR+TI } \\
\text { IOTAL }\end{array}$ & $\begin{array}{r}0 \\
190 \\
123 \\
190 \\
313\end{array}$ & $\begin{array}{r}-576 \\
-92 \\
41 \\
-669 \\
709\end{array}$ & $\begin{array}{l}0 \\
-6 \\
63 \\
-6 \\
70\end{array}$ & $\begin{array}{l}576 \\
211 \\
144 \\
695 \\
779\end{array}$ & $\begin{array}{r}-41 \\
6 \\
66 \\
-35 \\
101\end{array}$ & $\begin{array}{r}4 \\
-113 \\
24 \\
-109 \\
132\end{array}$ & $\begin{array}{r}160 \\
66 \\
90 \\
227 \\
317\end{array}$ & $\begin{array}{l}166 \\
131 \\
114 \\
254 \\
358\end{array}$ \\
\hline A11 & + & $\begin{array}{l}\text { GR } \\
\text { T1 } \\
\text { R1 } \\
\text { GR+T1 } \\
\text { TOTAL }\end{array}$ & $\begin{array}{r}0 \\
190 \\
67 \\
190 \\
257\end{array}$ & $\begin{array}{l}223 \\
-92 \\
18 \\
131 \\
149\end{array}$ & $\begin{array}{r}0 \\
-6 \\
37 \\
-6 \\
44\end{array}$ & $\begin{array}{l}223 \\
211 \\
78 \\
231 \\
300\end{array}$ & $\begin{array}{r}-41 \\
13 \\
8 \\
-28 \\
36\end{array}$ & $\begin{array}{r}4 \\
-74 \\
167 \\
-70 \\
238\end{array}$ & $\begin{array}{r}318 \\
-272 \\
46 \\
46 \\
92\end{array}$ & $\begin{array}{r}321 \\
283 \\
174 \\
89 \\
258\end{array}$ & A15 + & $\begin{array}{l}\text { GR } \\
T 1 \\
R 1 \\
\text { GR+TI } \\
\text { TOTAL }\end{array}$ & $\begin{array}{r}0 \\
190 \\
124 \\
190 \\
314\end{array}$ & $\begin{array}{r}-600 \\
-92 \\
42 \\
-693 \\
735\end{array}$ & $\begin{array}{l}0 \\
-6 \\
64 \\
-6 \\
70\end{array}$ & $\begin{array}{l}600 \\
211 \\
146 \\
718 \\
802\end{array}$ & $\begin{array}{r}-41 \\
6 \\
66 \\
-35 \\
101\end{array}$ & $\begin{array}{r}4 \\
-113 \\
24 \\
-109 \\
132\end{array}$ & $\begin{array}{r}160 \\
66 \\
90 \\
227 \\
317\end{array}$ & $\begin{array}{l}166 \\
131 \\
114 \\
254 \\
358\end{array}$ \\
\hline & & & & & & & & & & & & & & & & & ? & & & \\
\hline
\end{tabular}




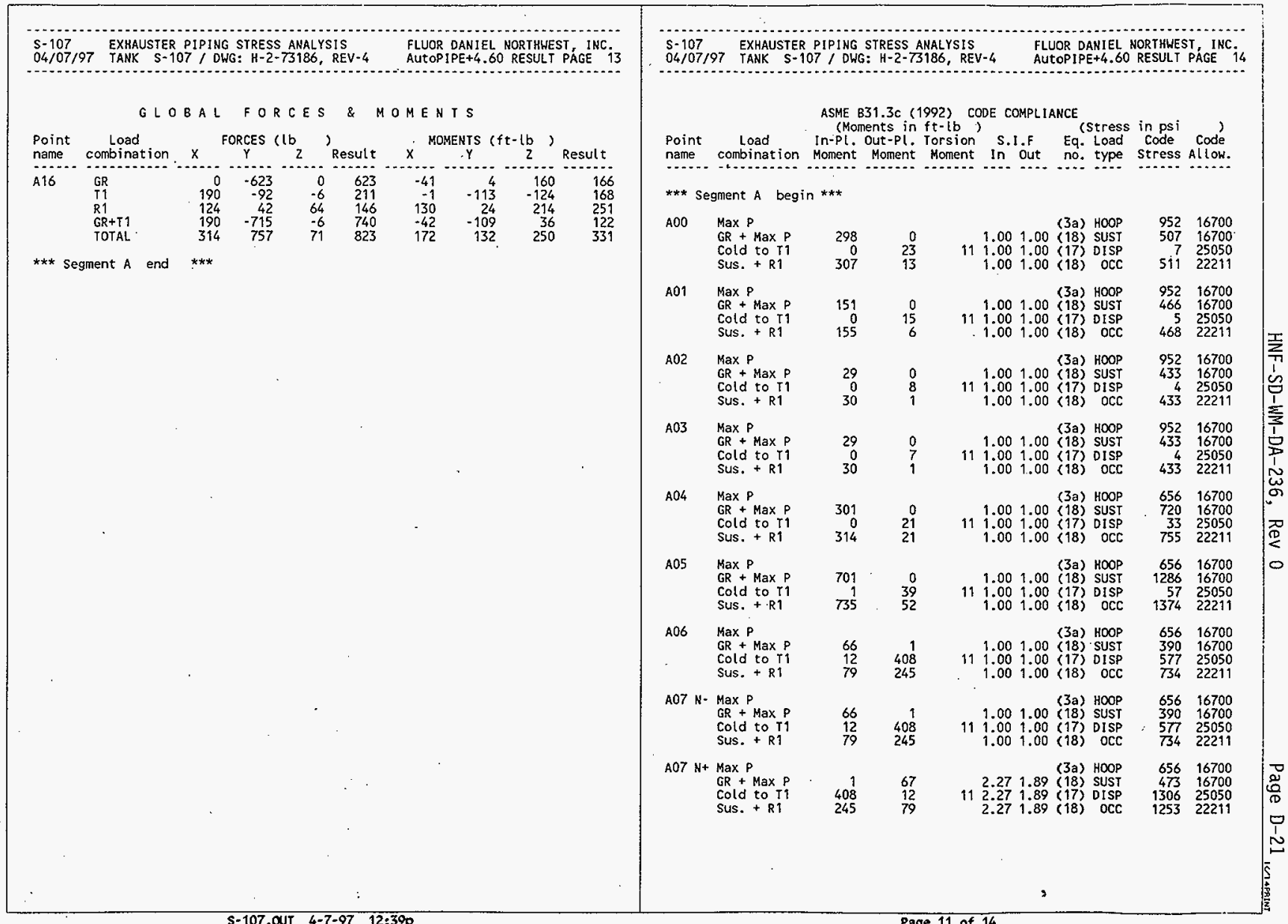




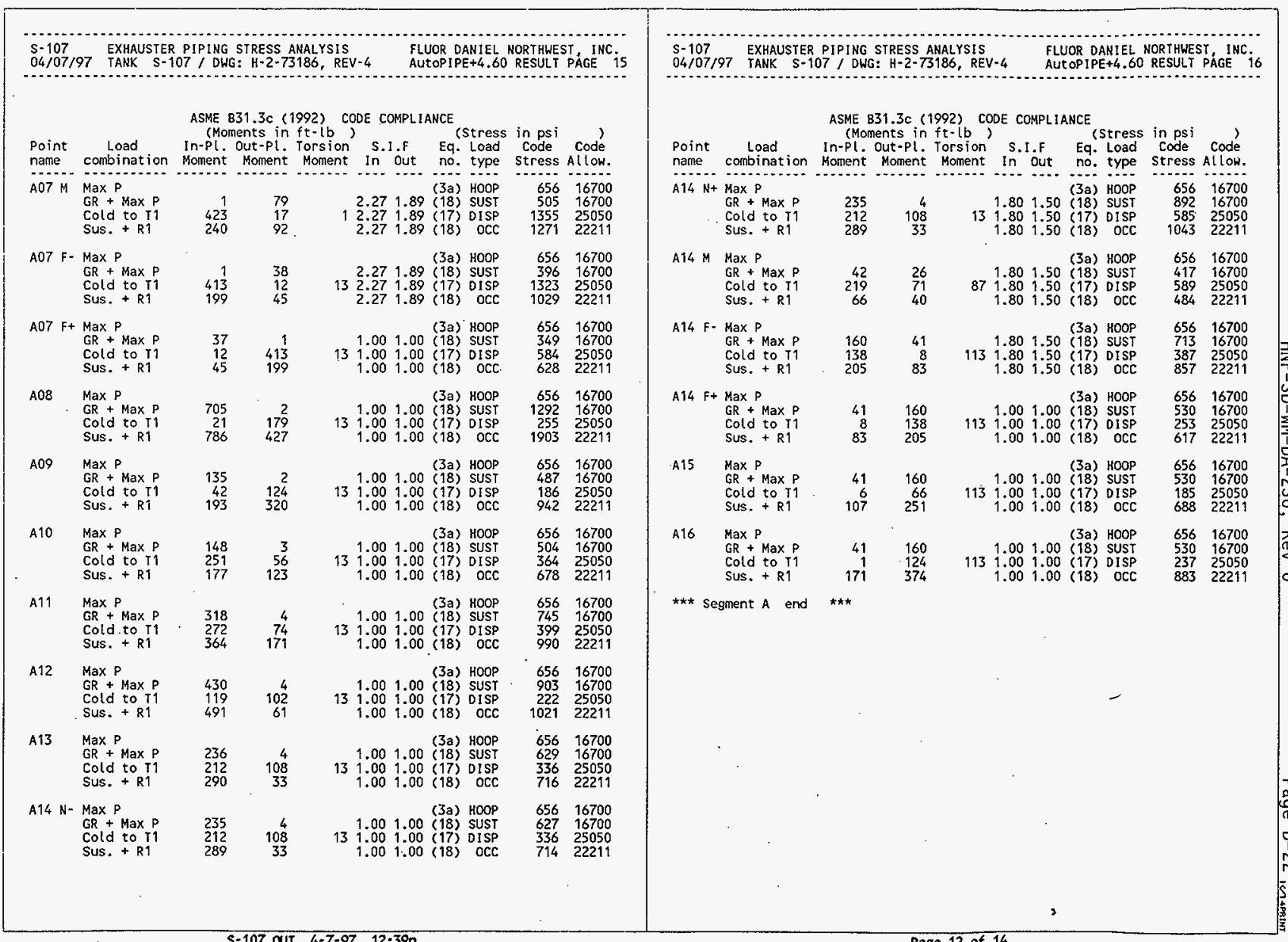




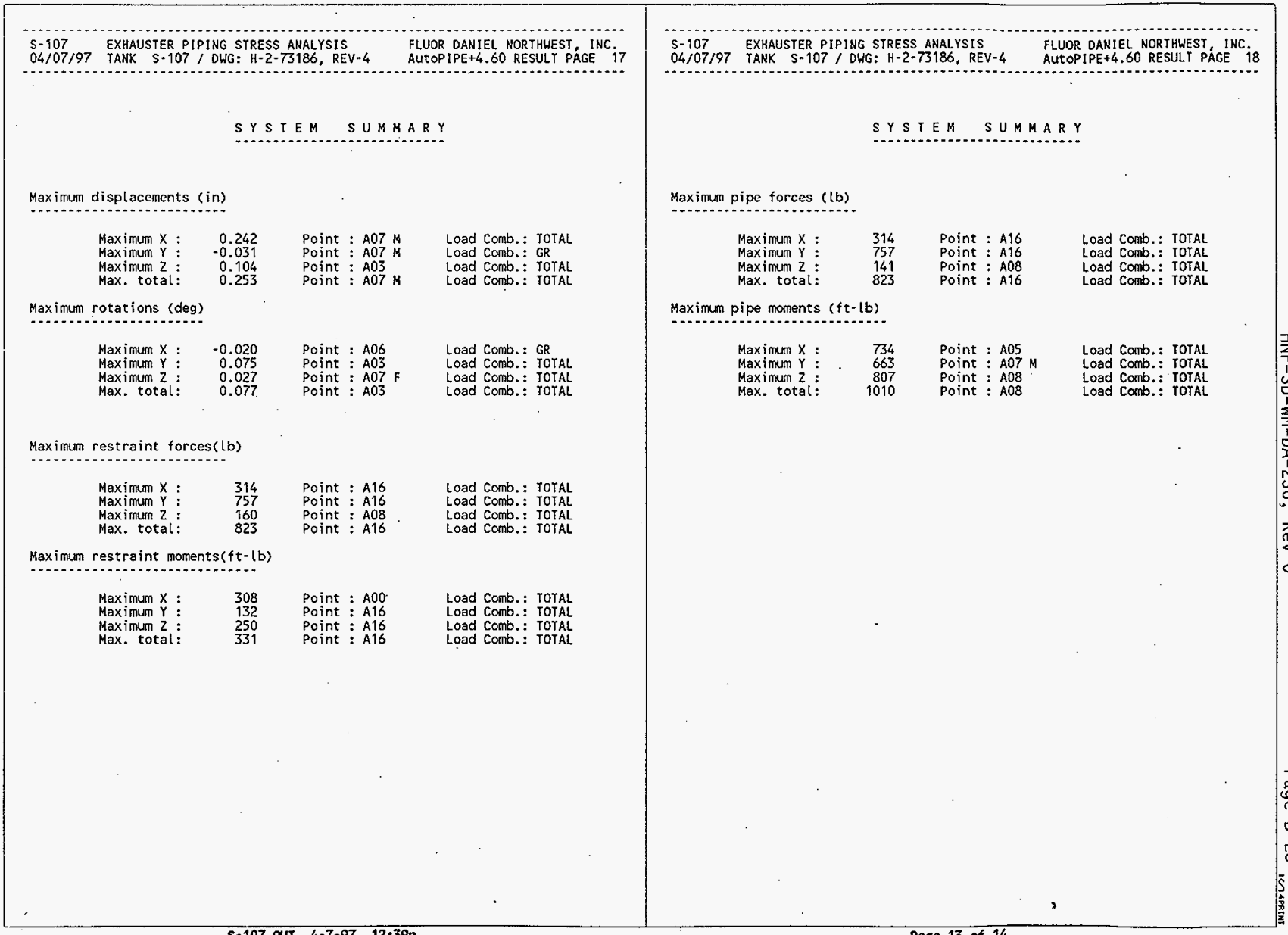




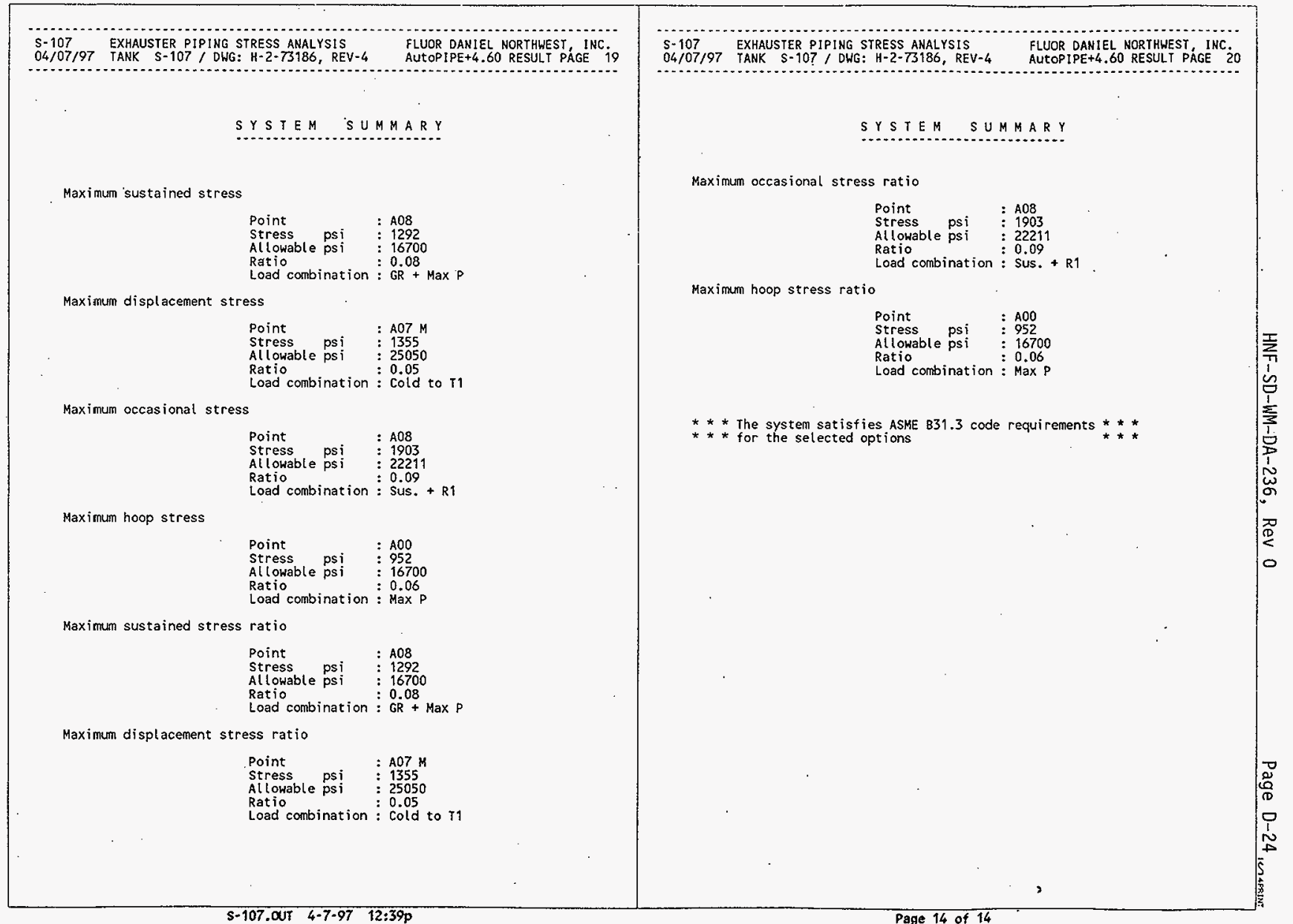


HNF-SD-WM-DA-236

Rev. 0

APPENDIX E: DESIGN CALCULATIONS FOR TANKS S-101/102 INSTALLATION 
Fluor Daniel Northwest, Inc.

CALCULATION IDENTIFICATION AND INDEX
Page 1 of 12

Date

May 7, 1997

This sheet shows the status and description of the attached Design Analysis sheets.

Discipline Mechanical

wo/Job No. E40690

Calculation No. M-5

Project No. \& Name 241-S-101/102 1000 CFM Exhauster Installation

Calculation Item

These calculations apply to:

Dwg. No. ECN 618795

Rev. No.

Dwg. No.

Rev. No.

other (Study, COR)

Rev. No.

The status of these calculations is:

[] Preliminary Calculations

[X] Final Calculations

[] Check Calculations (On Calculation Dated) .

[] Void Calculation (Reason Voided)

Incorporated in Final Drawings?

[] Yes [X] No

This calculation verified by independent "check" calculations?

[] Yes [] No

Original and Revised Calculation Approvals:

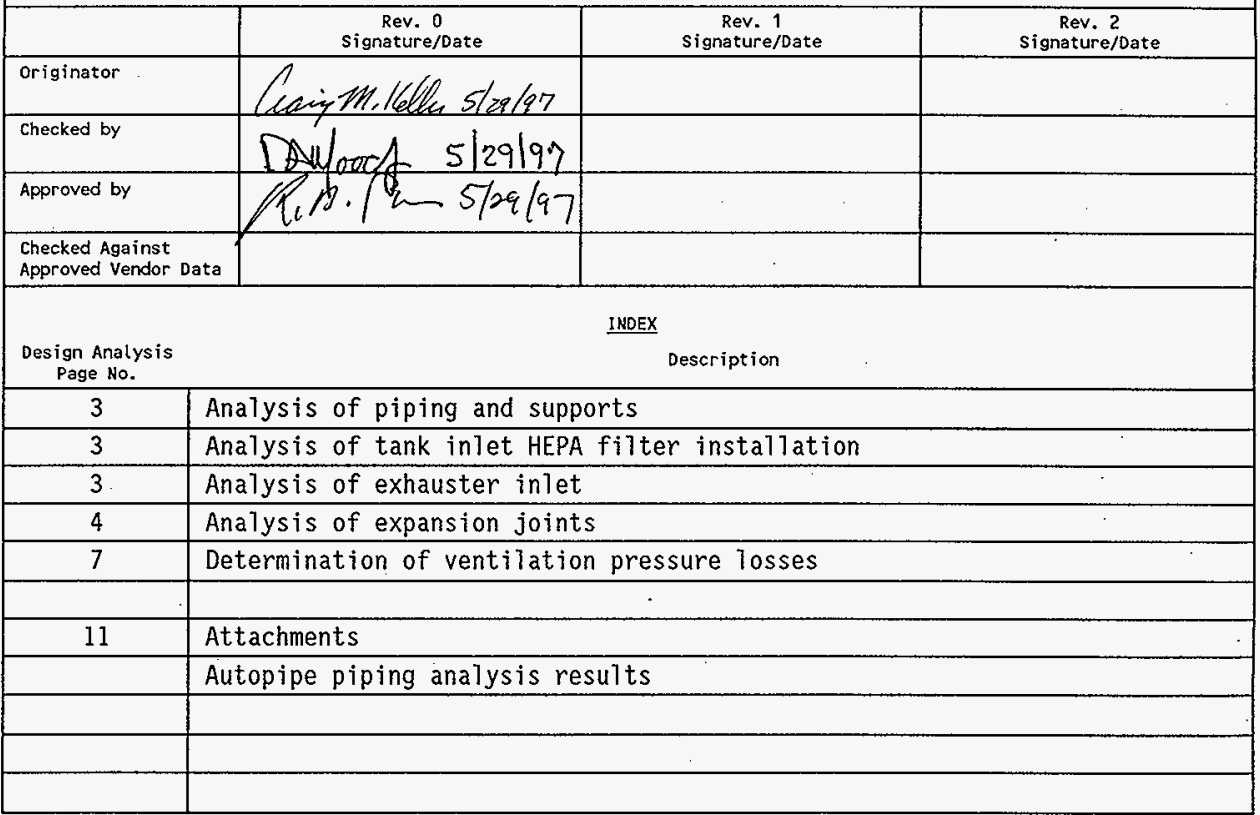


Fluor Daniel Northwest, Inc.

\section{DESIGN ANALYSIS}

ctient LMHC

subject S-101/102 Exhauster Installation

Location $241-5-101 / 102$
Calc. No. $M-5$

Revision 0

Page No. 2 of 10

\section{OBJECTIVE:}

Perform analysis of exhauster and HEPA filter installation on waste tanks 241-S101/102 to verify stresses are within acceptable values and to determine expected pressure drop in the ventilation ducting.

\section{CRITERIA:}

Ventilation and condensate piping are considered safety significant/safety class 2. Design loads for analysis are specified in Design Loads for Facilities GC-LOAD-01, Rev 1. Piping is analyzed using criteria specified in the ASME B31.3 Process Piping Code.

\section{ASSUMPTIONS/GIVENS:}

Piping stresses and support loads used in the analysis were determined using Autopipe and are provided in the Attachments section. Analysis results for comparable installation designs have been applied where possible. These other installations were analyzed in calculations $M-1$ through $M-4$ of the same Job Number.

\section{CALCULATION METHOD:} design.

Hand calculations and Autopipe analysis have been used to verify adequacy of the

\section{REFERENCES:}

1) 1993 ASHRAE Handbook Fundamentais, American Society of Heating, Refrigeration and Air-Conditioning Engineers, Inc. 
KAISER ENGINEERS

HANFORD

Client

Subject

Location
HNF-SD-WM-DA-236, Rev 0

DESIGN ANALYSIS
Page E-5

Calc. No. $\wedge-5$

Revision $s$

Page No. $y$ of $\angle 0$

WO/Job No. E 40690

Date $5-7-97$ By CM KELLER

Checked $5-28-92$ By $D A$ MooDs

WIND COADING ANO MISSILE IMPACT HAZARTS HAVE. BEEN SATISFACTRILY ANALYZES IN CALCULATION M-Z, FURTHER ANALSSIS FOR THIS INSTALLATTON IS NOT... JUSTIFIED.

CHECK DISPLACEMENTS FT EXTANSION JUINT LOCPTONS: EXtRUSTER NLET

EXP. TOINT - PATHUAAY IZCSFFSOBC-MI $\begin{aligned} \text { ALLOWABLE DISPLACEMENTS: } A X I A< & 3.28^{\prime \prime} \\ \text { LATERAL } & .55^{\prime \prime} \\ \text { ANKULAR } & 10^{\circ} \\ \text { TORSIONAL } & 173^{\circ}\end{aligned}$

ACTUAL TPISPLACEMENTS AT TOINT BOZ

\begin{tabular}{|c|c|c|c|c|}
\hline TTEANSLATIONS & $x$ & $.0 z z^{\prime \prime}$ & (LATERAL) & $O K$ \\
\hline & y & $.015^{\prime \prime}$ & ( $\angle A T E R A L)$ & ok \\
\hline & $z$ & $.046 "$ & $(A X / A L)$ & $O K$ \\
\hline ROTATIONS & $x$ & $.0244^{\circ}$ & (ANCULAR) & OK \\
\hline & $y$ & $.045^{\circ}$ & (ANCUULAR) & $D$ \\
\hline & $z$ & $.020^{\circ}$ & (TORSIONAL) & $\Delta K$ \\
\hline
\end{tabular}

$\therefore$ ACOUAC DISPLACEMENTS FOR EXPANSION TOINT AT EXHAUSTER WLET ARE WITHW ALCOWABLES. 


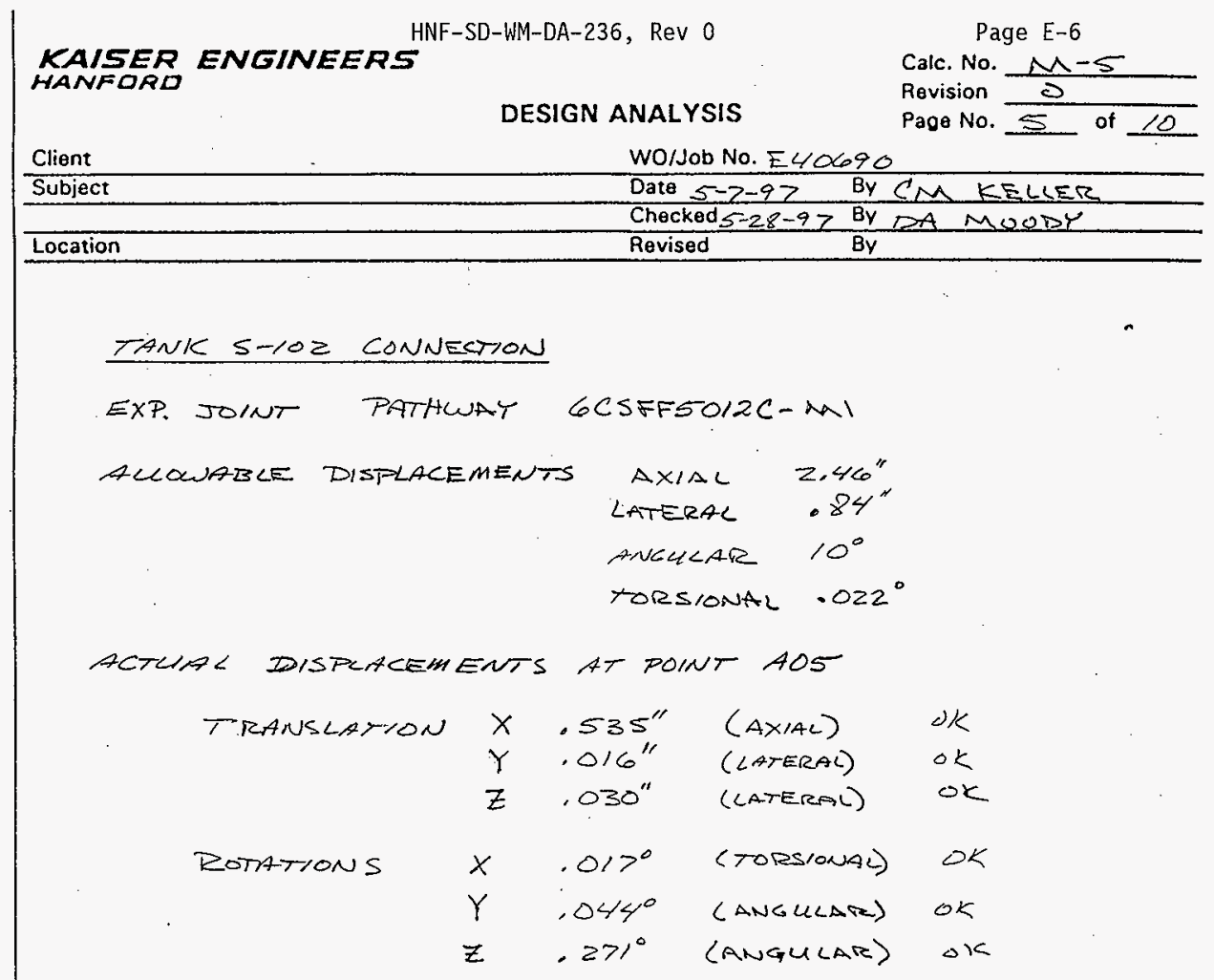

$\therefore$ ACTUAC DISPLACEMENTS FOR EXTANSION JOINTAT TANK SHOZ CONNECTION ARE WITHIN ALLOWABLES.

TANK S-1O/ CONNECTION

EXP TOINT IS SAME AS S-102 CONNECTION

ACTUAL DISFLACEMENTS AT DOINT AZZ

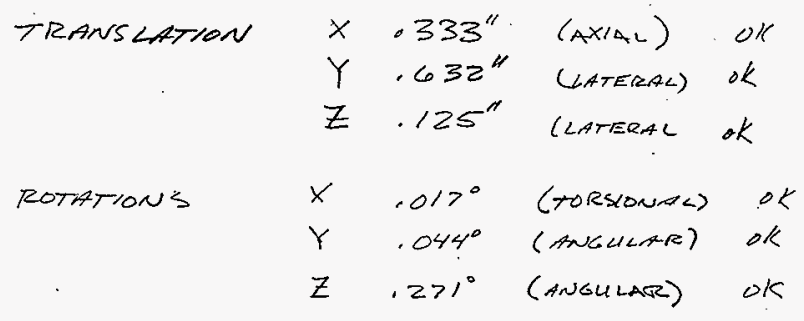

54.4300-037 KEH-0037.00 (06/92) 


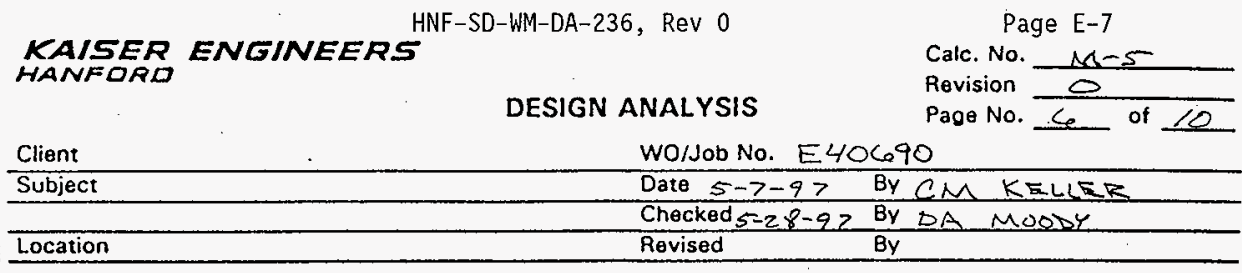

DISPLACEIIENTS FOR THE EXPANSION JOINT AT THNK 5-10, CONNECTION ARE WITHIN ALCOWABSLES AS ANALYZED, FLOWING COMPLETION OF THE AUTOPIPE ANALYSIS. AN ADDITIONAL SUPPORT WAS ADDED TO REDUCE VERTICAL. DISPLACEMENTS. ACTUAL VERTICAL DISPLACEMENTS ARE GONE TO BE LESS AND ARE EXPECTED TO BE SIMILAR TO VALUES FOE THE TANK SHOD CONNECTION, THE ADDED SUPPORT HAS NO OTHER SIGNIFICANT IMPACT ON THEE ANALYSIS RESULTS.

$54-4300-037 \quad \mathrm{KEH}-0037.00(06 / 92)$ 
KAISER ENGINEERS

HANFORO

HNF-SD-WM-DA-236, Rev 0

DESIGN ANALYSIS
Page E-8

Calc. No. M-S

Revision $\Delta$

Page No: $z$ of 10
Client

Subject

Location
WO/Job No. Estoc90

Date $5-7-97$ By CM KELCER

Checked 5-28-97 8y ph $4000 \%$

By

DETERMINE VENTILATHON PRESSURE DROF FROM ATMOSPHERE TO EXHAUSTER INLET. ASSUME PRESSURE

DROP OF, "WIG, ACROSS TANK MNLT HEPA FILTER. USE FLOW RATE OF SDO CFM PER TANK, USE SATHRATED AIE AT 140'F AS THE MEDIUM TO BE EXHAUSTED. CONSIDER THE FOLLOWINK LOSSES.

A) LOSS FROM ATMOSPHERE TO THWK DOME SPACE:

$$
\Delta P_{\text {NUET }}=1.34 \text { in ars naf Calculation m-4 }
$$

B) LOSS FROM TANK DOME SFACE TO 6"PLENUM:

THE TRUK IS EXHALSTEO THROUGH A $\phi$ ZU"

DPENINL INTO A $3^{\prime} \times \sigma^{\prime}$ CONDENSOR PIT. LOSSES

FOE SUCH LARGE OPENINCS ARE NEGLIGIBLE FOR THE

SOO FM FLOW ZATES AND WIL THEREFORE BE

NEGLECTED.

C) LOSS IN $\not 6^{\prime \prime}$ PLENUM FROM TANK S-10,

ENTRANKE: LOSS $L_{c}=19^{\prime}$

$6^{\prime \prime} \angle R E L B O W \quad \angle e=10^{\prime}$

$6^{\prime \prime} B F L Y$ VALVE Le $=23^{\prime}$ eST

$6 "$ PIPE, 27'LENGTH $L=27^{\prime}$

6"X12" EXPANSION $L_{e}=2 z^{\prime}$

$707 A<L_{e}=10 \prime^{\prime}$ 


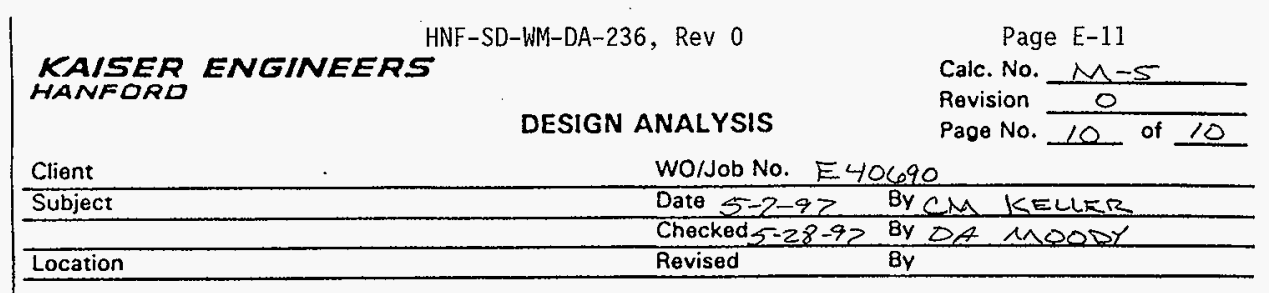

TOTAL PRESSURE LOSS FROM ATMOSTHERE TO EXHAUSTER INLET FOR TANK $S-10$, IS:

$$
\begin{aligned}
& \Delta p=1,34 \text { in wot }(10, x) 1,19 \text { incog/100 })+(60)\left(.04 \text { in } \frac{w 6}{100}\right)+(301)(.15) \text { in w } 100
\end{aligned}
$$

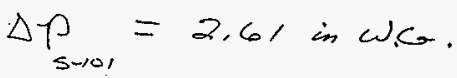

PTRESSURE LOSS FOR THNK S MOZ is:

$$
\begin{gathered}
\Delta p=1.34+\left(149^{\prime}\left(1.19 / 100^{\prime}\right)+\left(60^{\prime}\right)\left(.04 / 100^{\prime}\right)+\left(30^{\prime}\right)\left(.157 / 100^{\prime}\right)\right. \\
\Delta p_{\text {soz }}=3.18 \text { in w.G. }
\end{gathered}
$$

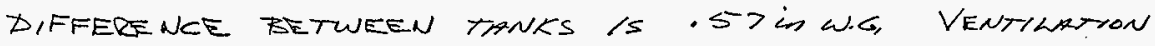
BALANCING BETWEEN TIONKS SHOWLD WOT BER PROBLEM.

54-4300-037 KEH-0037.00 106/92) 


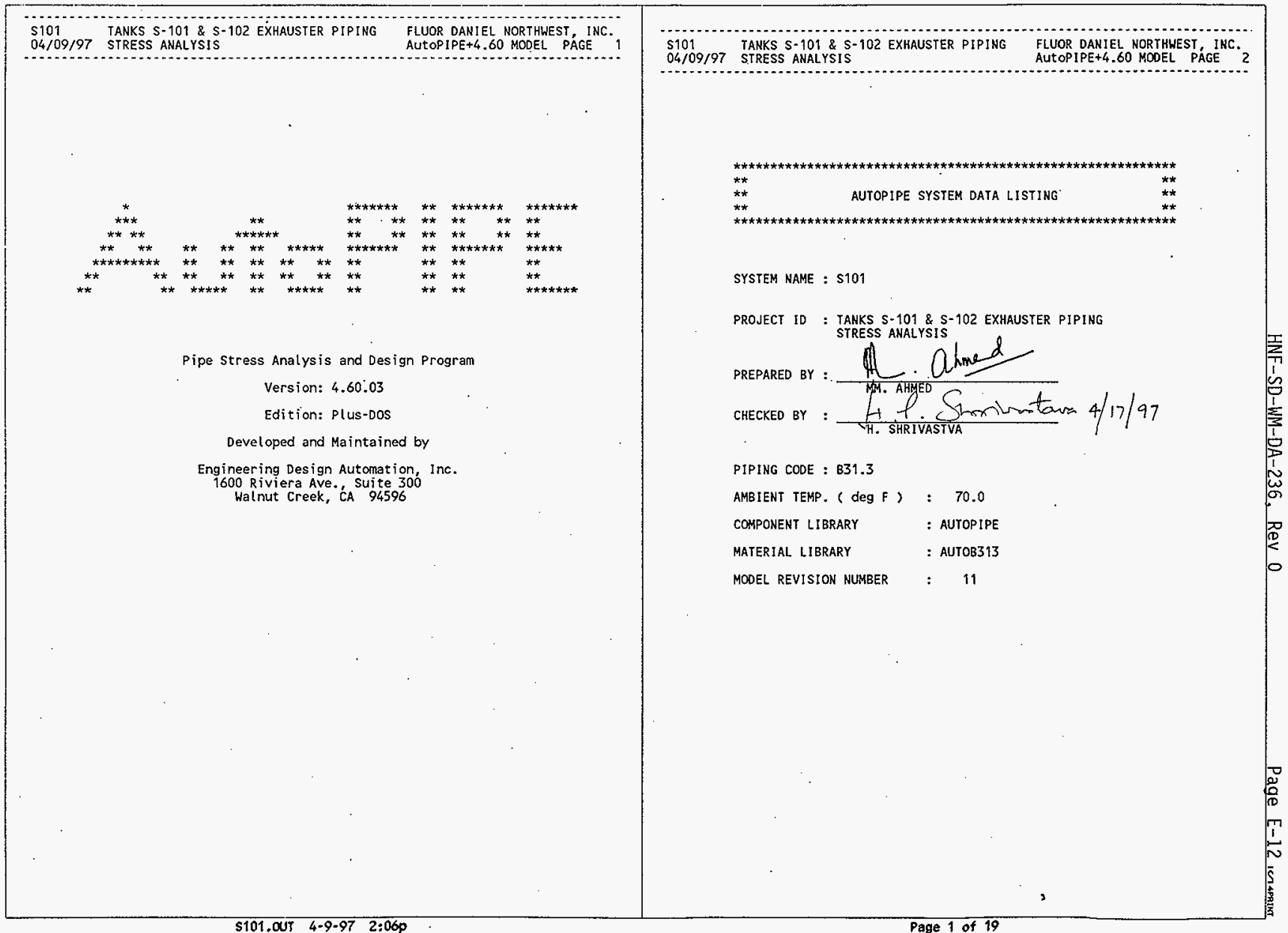




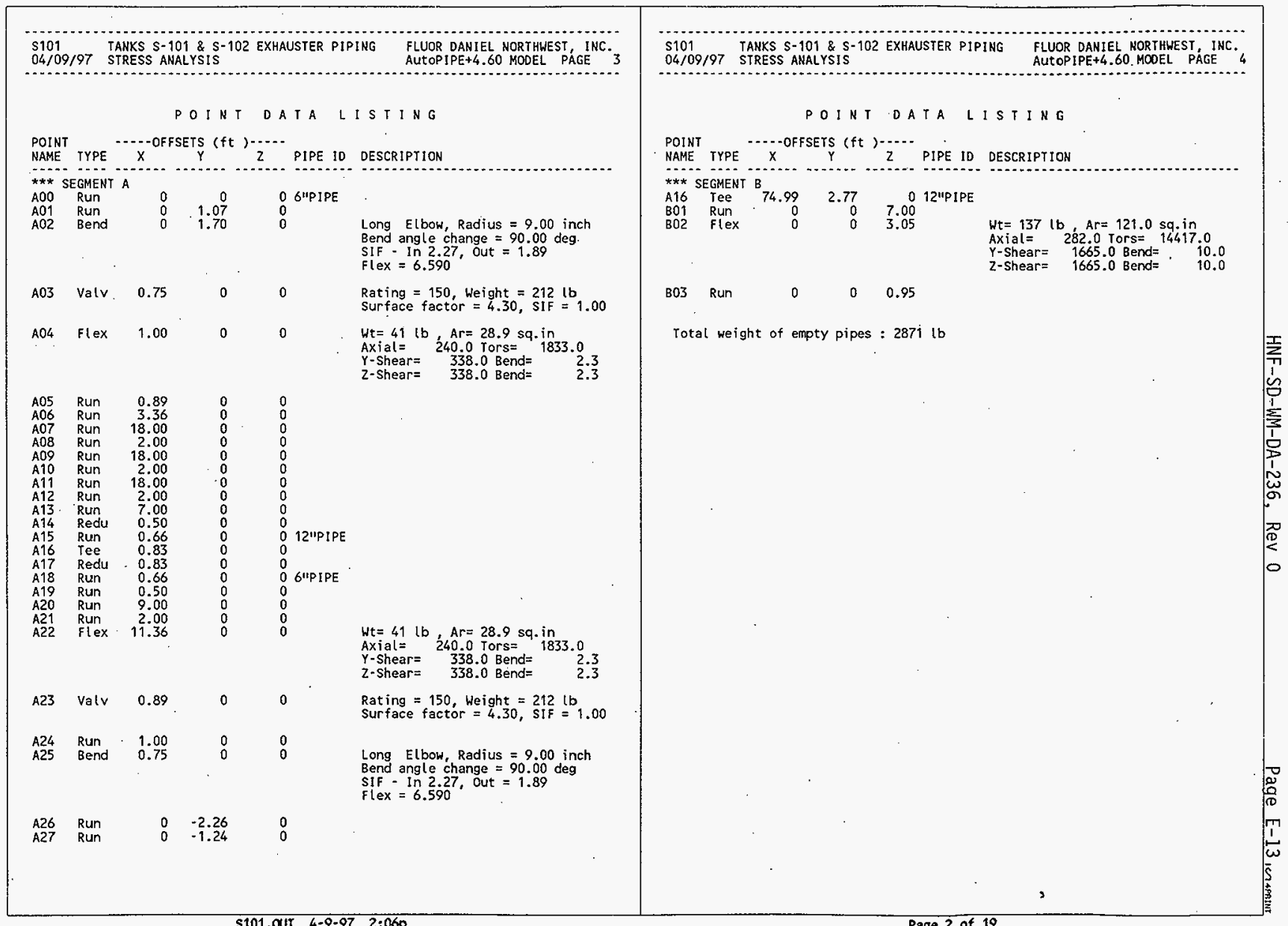




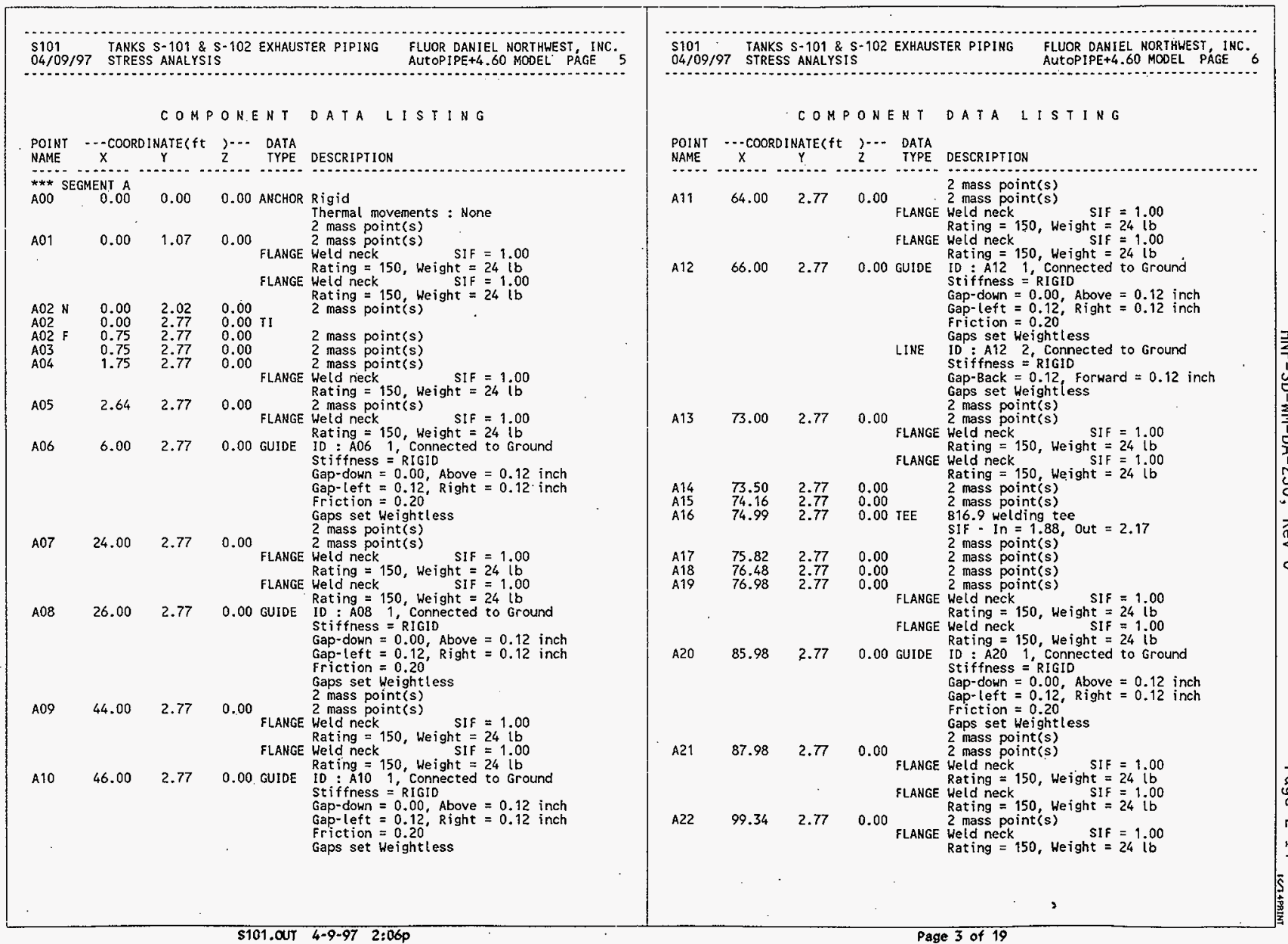




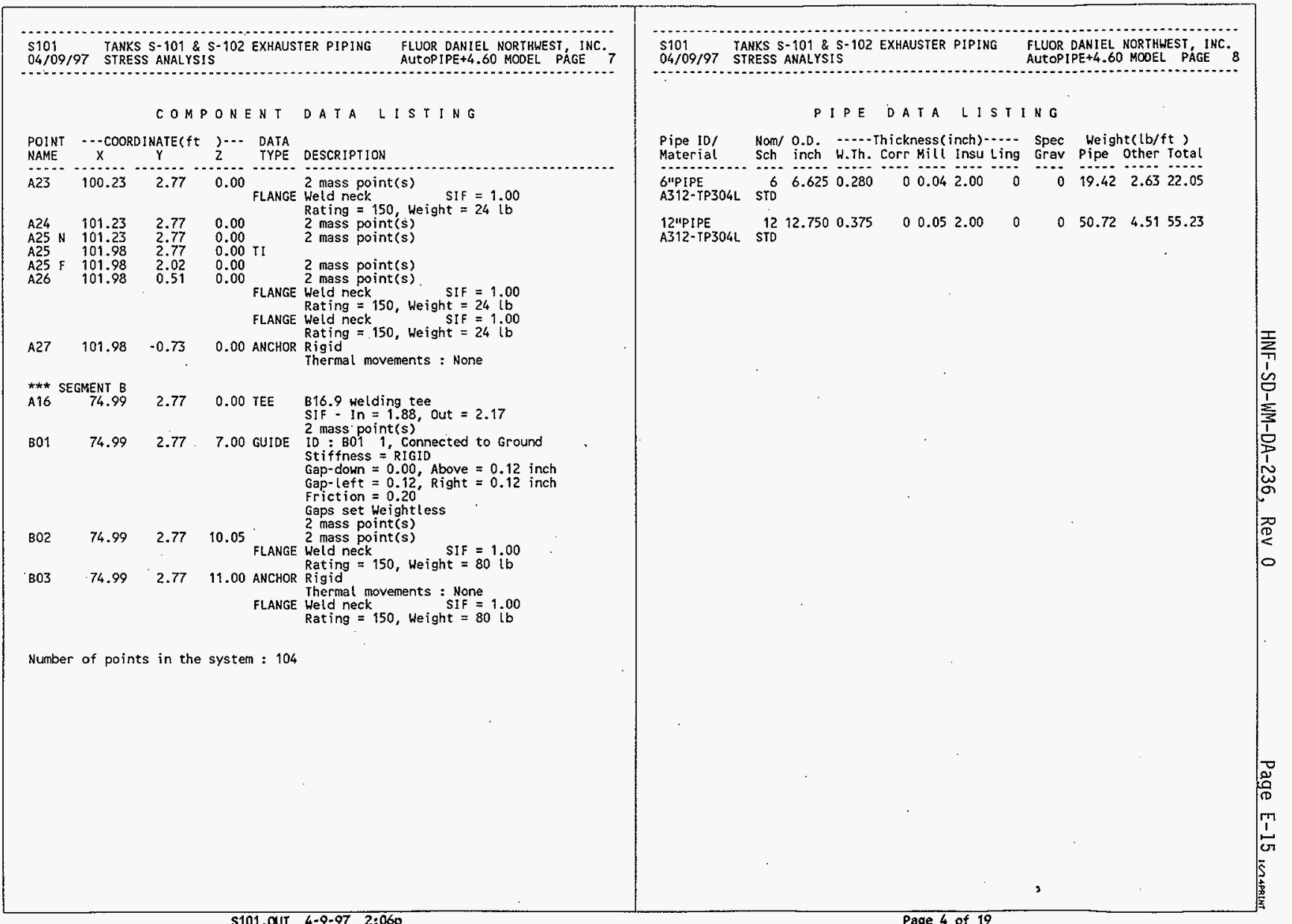




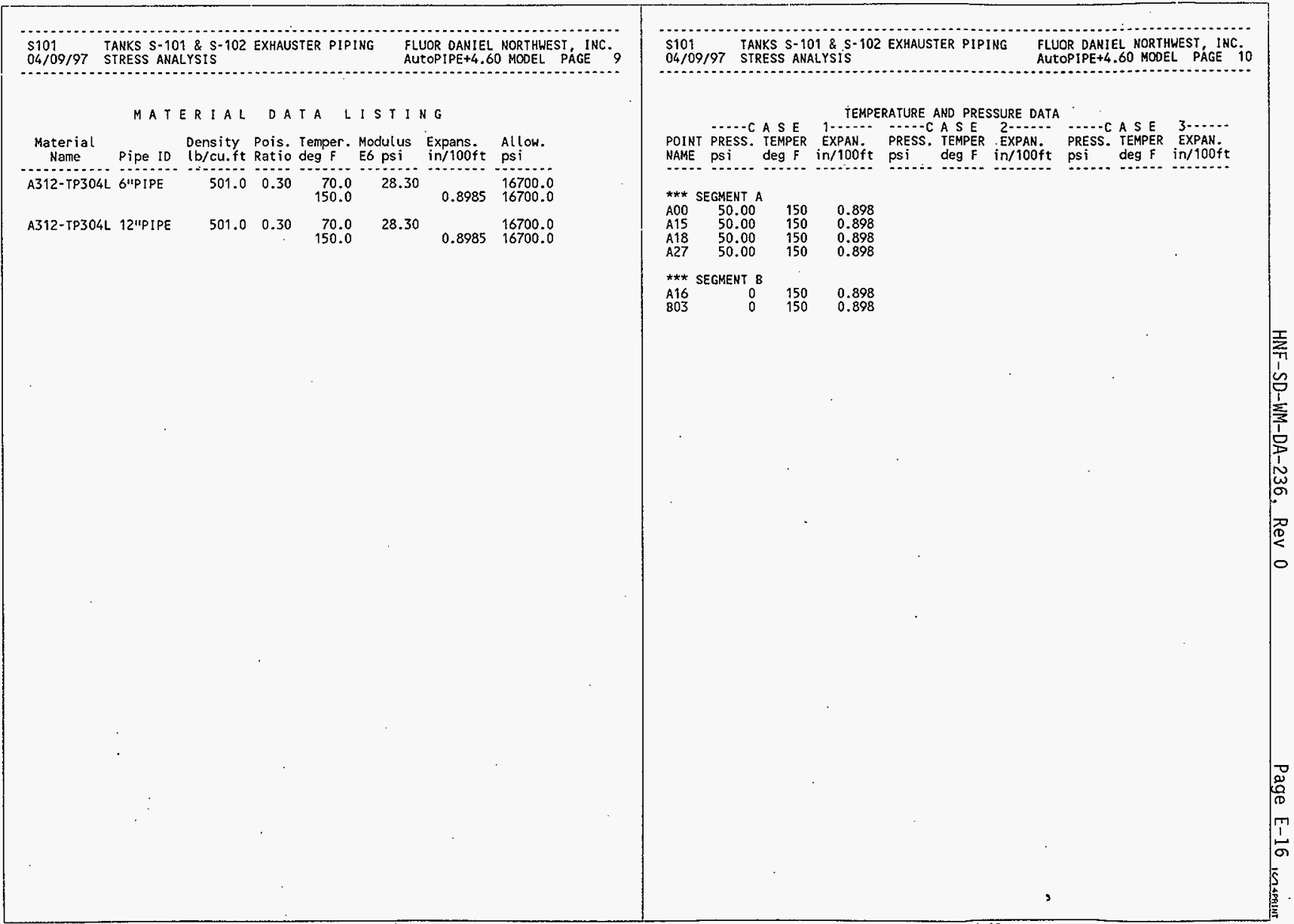




$\begin{array}{ll}\$ 101 & \text { TANKS } S-101 \& S-102 \text { EXHAUSTER PIPING } \\ 04 / 09 / 97 & \text { FLUOR DANIEL NORTHWEST INC. } \\ \text { AUTOPIPE+4.60 RESULI PÁGE }\end{array}$

STRESS ANALYSIS

\section{A NALYSIS SUMMARY}

Current model revision number : 11

Static - Date and Time of analysis Model Revision Number

Number of load cases

Apr 9, $1997 \quad 2: 05$ PH (1)

GR T1

rolerance - Force, Displacement ......... 100.00 lb 0.0162 in

Friction - Factor, tolerance ............ $1.00 \quad 0.10$

Hanger design run'.................... No

Cut short included ................... No

occasional load analysis type .......... Nonl inear

Non-linear analysis sumary file ....... s101.LoG

Base load cases for nonlinear analys is

Weight of contents included.

$G R=N$ one

Number of mass points per span.......... Yes

Pressure stiffening case ..............

Water elevation for buoyancy loads ..... Not considered

Modal - Date and Time of analysis ............. Apr 9, 1997 2:05 PM Model Revision Number .................. Apr 11

Number of modes $\ldots \ldots \ldots \ldots \ldots \ldots \ldots \ldots, 52$

Cutoff frequency (Hz) $\ldots \ldots \ldots \ldots \ldots$. 100.0

Weight of contents included ........... Yes

Number of mass points per span ......... 2

Pressure stiffening case $\ldots \ldots$.........
Water elevation for buoyancy loads $\ldots$... Not considered

Response - Date and $\mathrm{T}$ ime of analysis ............ Apr 9, 1997 2:06 PM Model Revision Number ................... 11

Number of load cases $\ldots \ldots \ldots \ldots \ldots \ldots \ldots 1$

Load cases analyzed Date and time of modal anaiysis........... R1 Apr 9, $1997 \quad 2: 05$ PM

Number of modes ...................... 52

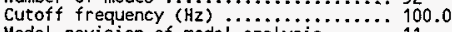

Model revision of modal analysis ....... 11

Weight of contents included ........... Yes

Number of mass points per span ........?

Pressure stiffening case ..............

Water elevation for buoyancy loads .... Not considered
S101 TANKS $S-101 \&$ S-102 EXHAUSTER PIPING

04/09/97 STRESS ANALYSIS FLUOR DANIEL NORT
AUTOPIPE+4.60 RESULT PAGE 2

CODE COMPLIANCE COMBINATIONS

\begin{tabular}{|c|c|c|c|c|c|c|}
\hline Combination & Category & Method & Load & Factor & Allowable & Remarks \\
\hline$G R+\operatorname{Max} P$ & Sustain & sum & $\begin{array}{l}\text { Gravity } \\
\text { Max Long }\end{array}$ & $\begin{array}{l}1.00 \\
1.00\end{array}$ & Automatic & Default \\
\hline Cold to T1 & Expansion & Sum & Thermal 1 & 1.00 & Automat ic & Default \\
\hline Sus. + R1 & accasion & Abs sum & $\begin{array}{l}\text { Response } 1 \\
\text { Max Sus }\end{array}$ & $\begin{array}{l}1.00 \\
1.00\end{array}$ & Automat ic & Default \\
\hline $\operatorname{Max} P$ & Hoop & & Max Hoop & 1.00 & Automat ic & Defau \\
\hline
\end{tabular}

OTHER USER COMBINATIONS

$\begin{array}{lllll}\text { Combination } & \text { Method } & \text { Load } & \text { Factor } & \text { Renarks } \\ \text { GR } & \text { Sum } & \text { Gravity } & 1.00 & \text { Default } \\ T 1 & \text { Sum } & \text { Thermal } 1 & 1.00 & \text { Default } \\ \text { R1 } & \text { Sum } & \text { Response } 1 & 1.00 & \text { Default } \\ \text { GR+T1 } & \text { Sum } & \begin{array}{l}\text { Gravity } \\ \text { Thermal } 1\end{array} & 1.00 & \text { User } \\ & \text { Abs sum } & \text { GR+T1 } & 1.00 & \text { User } \\ \text { TOTAL } & \text { Abs } & & \end{array}$

\section{CODE COMPLIANCE}

$Y=$ Factor $\ldots \ldots \ldots \ldots \ldots \ldots \ldots \ldots \ldots \ldots . .40$ Weld efficiency factor .............. 1.00 Range reduction factor $\ldots \ldots \ldots \ldots \ldots \ldots .1 .00$ Design Pressure Factor ...............1.00 Minimum stress ratio used in reports... 0.00 Include corrosion in stress calcs. .... Y include axial force in code stress .....

longi tudinal pressure calculation ...... PO/4t

Include rigorous pressure ............. Not analyzed 


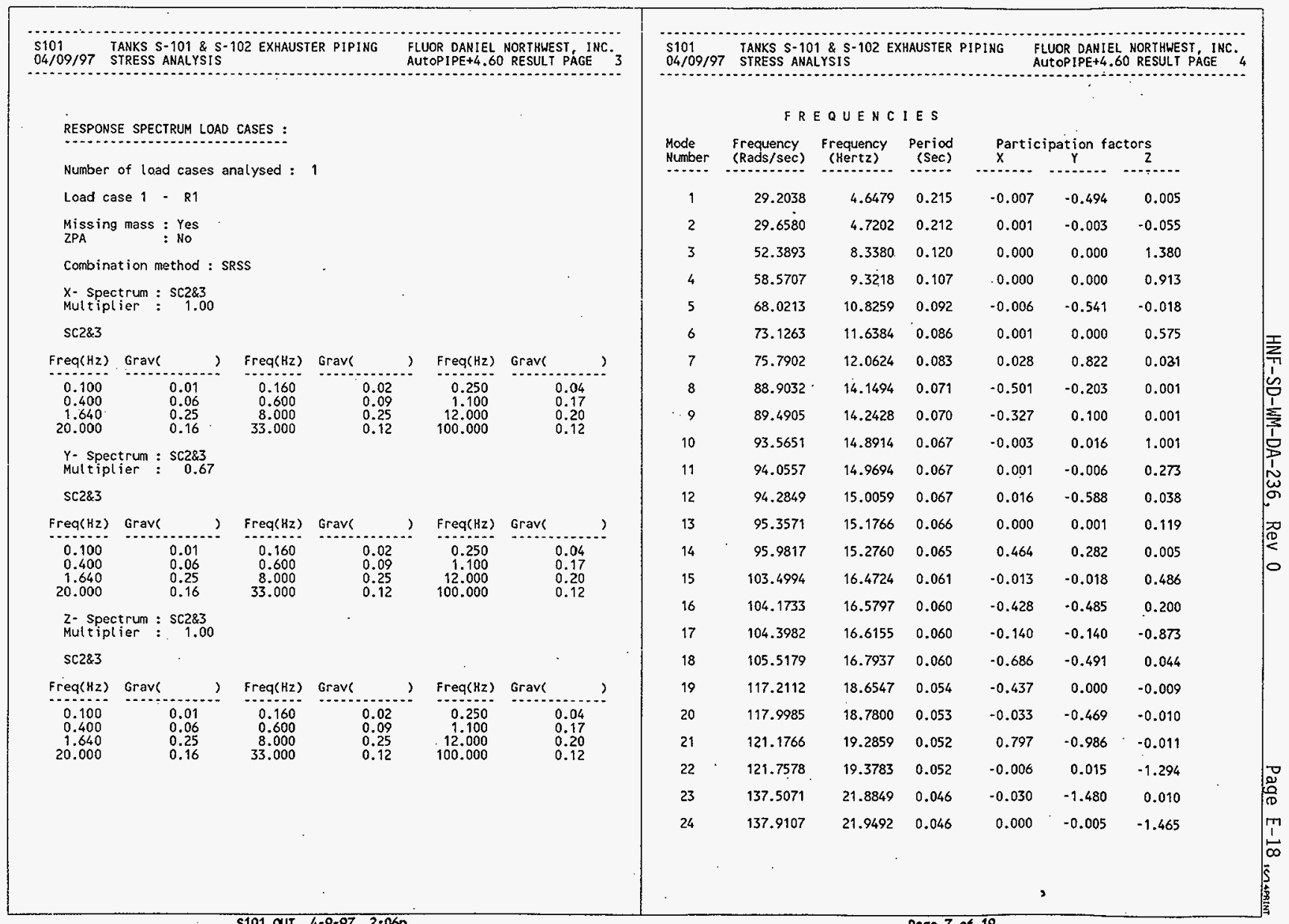




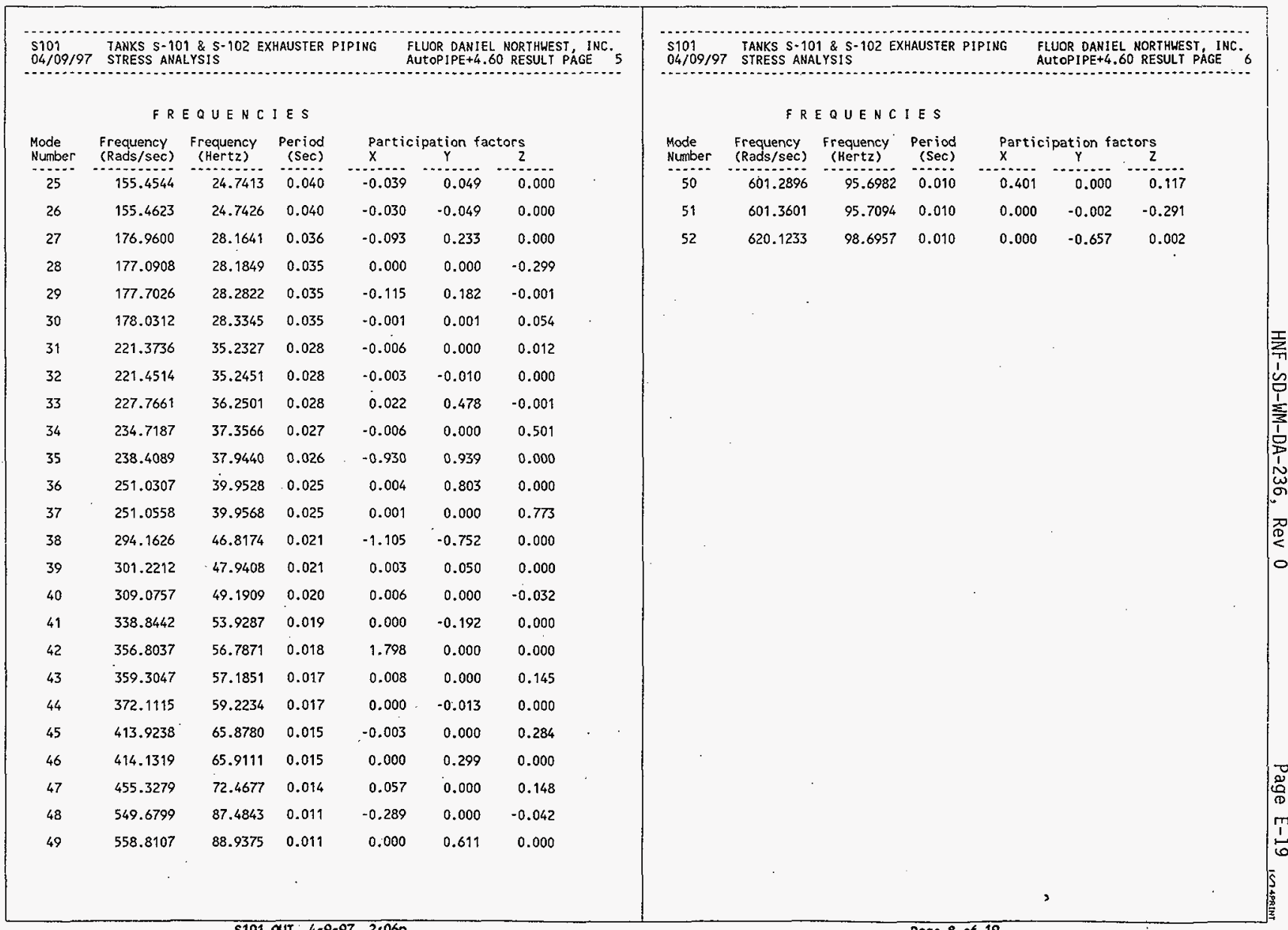




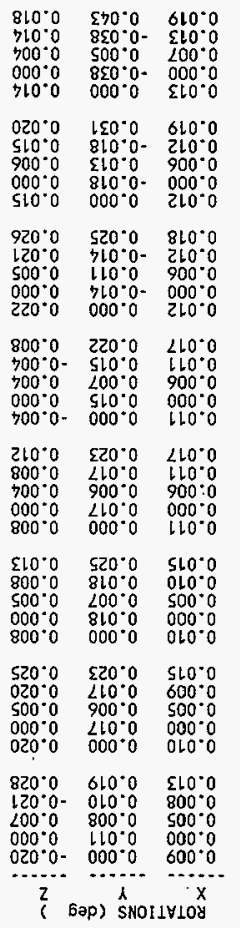

$860^{\circ} 0 \quad 120^{\circ} 0 \quad \angle 60^{\circ} 0$ $080^{\circ} 0-210^{\circ} 0 \quad \angle 60^{\circ} 0$ $\begin{array}{lll}080^{\circ} 0- & 000^{\circ} 0 & 800^{\circ} 0\end{array}$ $000^{\circ} 0$ \&10.0 $200^{\circ} 0^{\circ}$

$021.0 \quad 000.0 \quad 750.0$ $021^{\circ} 0-000^{\circ} 0$ + $\$ 20^{\circ}$

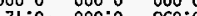
$\begin{array}{lll}12 l^{\circ} 0^{-} & 000^{\circ} 0 & 950^{\circ} 0 \\ 100.0 & 000^{\circ} 0 & 200^{\circ} 0 .\end{array}$

zEL 0 010.0 $910^{\circ} 0$ $\angle 21^{\circ} 0-800^{\circ} 0-910^{\circ} 0$ 131.0 . $000^{\circ} 0 \quad 810^{\circ} 0$ $\begin{array}{lll}221^{\circ} 0^{\circ} & 000^{\circ} 0 & 810^{\circ} 0 \\ & 800^{\circ} 0- & 200^{\circ} 0 .\end{array}$

$02 L^{\circ} 0 \quad 000^{\circ} 0.97 l^{\circ} 0$

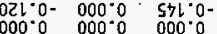
$1 \mathrm{i} \cdot 0-000 \% 000$. $\begin{array}{lll}L 21.0- & 000^{\circ} 0 & 27 l^{\circ} 0-\end{array}$

$911^{\circ} \quad \varepsilon 00^{\circ} 0$ 791.0 $211^{\circ} 0-100^{\circ} 0-\varepsilon 9 l^{\circ} 0-$ $511^{\circ} 0-000^{\circ} 0$ 291.0.

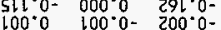

$850^{\circ} 0 \quad 000^{\circ} 0$ SटE.

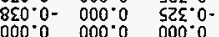

$650^{\circ} 0.000^{\circ}$ हटा.

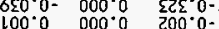

$\rightarrow \varepsilon 0^{\circ} 0 \quad 600^{\circ} 0 \quad \Sigma 7 \Sigma^{\circ} 0$

$150^{\circ} 0-900^{\circ} 0-\varepsilon \geqslant \varepsilon 0^{\circ}-$

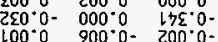

$\begin{array}{lll}\angle L 0^{\circ} & 000^{\circ} 0 & 505^{\circ} 0 \\ \angle 10^{\circ} & 000^{\circ} 0 & 705^{\circ} 0-\end{array}$

$\begin{array}{lll}210^{\circ} 0 & 000^{\circ} 0 & 705^{\circ} 0- \\ 000^{\circ} 0 & 000^{\circ} 0 & 000^{\circ} 0\end{array}$

$910^{\circ} 000^{\circ} 0$ SOS.0-

$100^{\circ} 0000^{\circ} 0200^{\circ} 0$ -

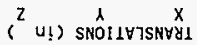

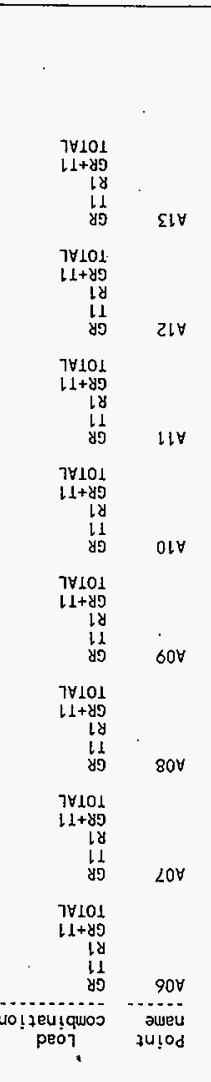

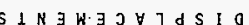

\begin{tabular}{|c|c|}
\hline $\begin{array}{l}120^{\circ} 0 \\
710^{\circ} 0^{-} \\
100^{\circ} 0 \\
000^{\circ} 0 \\
710^{\circ} 0\end{array}$ & $\begin{array}{l}610^{\circ} 0 \\
010^{\circ} 0 \\
600^{\circ} 0 \\
010^{\circ} 0 \\
000.0\end{array}$ \\
\hline $\begin{array}{l}060^{\circ} 0 \\
080^{\circ} 0- \\
010^{\circ} 0 \\
200^{\circ} 0 \\
280^{\circ} 0^{\circ}\end{array}$ & $\begin{array}{l}600^{\circ} 0 \\
000^{\circ} 0 \\
600^{\circ} 0 \\
000^{\circ} 0 \\
000^{\circ} 0\end{array}$ \\
\hline $\begin{array}{l}060^{\circ} 0 \\
080^{\circ} 0^{\circ} \\
010^{\circ} 0^{\circ} \\
200^{\circ} 0 \\
280^{\circ} 0^{-}\end{array}$ & $\begin{array}{l}600^{\circ} 0 \\
000^{\circ} 0 \\
600^{\circ} 0 \\
000^{\circ} 0 \\
000^{\circ} 0\end{array}$ \\
\hline $\begin{array}{l}060^{\circ} 0 \\
080^{\circ} 0 \\
010^{\circ} 0 \\
200^{\circ} 0 \\
280^{\circ} 0^{\circ}\end{array}$ & $\begin{array}{l}600^{\circ} 0 \\
000^{\circ} 0 \\
600^{\circ} 0 \\
000^{\circ} 0 \\
000^{\circ} 0\end{array}$ \\
\hline $\begin{array}{l}220.0 \\
610^{\circ} 0^{\circ} \\
700.0 \\
2000^{\circ} 0 \\
020^{\circ} 0^{-}\end{array}$ & $\begin{array}{l}700^{*} 0 \\
000^{\circ} .0 \\
700^{\circ} 0 \\
000^{\circ} 0 \\
000^{\circ} .0\end{array}$ \\
\hline $\begin{array}{l}2 \angle 0^{\circ} 0 \\
0 \angle 0^{\circ} 0 \\
200 \% \\
100^{\circ} 0 \\
110^{\circ} 0\end{array}$ & $\begin{array}{l}200^{\circ} 0 \\
000^{\circ} 0 \\
200^{\circ} 0 \\
000^{\circ} 0 \\
000^{\circ} 0\end{array}$ \\
\hline 000 & $\begin{array}{l}000^{\circ} \cdot 0 \\
000^{\circ} 0 \\
000^{\circ} 0 \\
000^{\circ} 0 \\
000^{\circ} 0^{0}\end{array}$ \\
\hline
\end{tabular}

\begin{tabular}{|c|c|c|}
\hline 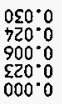 & $\begin{array}{l}910^{\circ} 0 \\
110^{\circ} 0 \\
500^{\circ} 0 \\
000^{\circ} 0 \\
110^{\circ} 0\end{array}$ & $\begin{array}{l}\text { SES } 0^{\circ} \\
7 E 5^{\circ} 0- \\
000^{\circ} 0 \\
\text { } E 5^{\circ} 0- \\
200^{\circ} 0^{\circ}\end{array}$ \\
\hline $\begin{array}{l}S 00^{\circ} 0 \\
000^{\circ} 0^{0} \\
500^{\circ} 0^{0} \\
000^{\circ} 0^{\circ} \\
000^{\circ} 0^{\circ}\end{array}$ & $\begin{array}{l}500^{\circ} 0 \\
200^{\circ} 0^{-1} \\
500^{\circ} 0 \\
920^{\circ} 0 \\
820^{\circ} 0^{-}\end{array}$ & $\begin{array}{l}620^{\circ} 0 \\
220^{\circ} 0 \\
200^{\circ} 0 \\
510^{\circ} 0 \\
210^{\circ} 0^{0}\end{array}$ \\
\hline $\begin{array}{l}\Sigma 00^{\circ} 0 \\
000^{\circ} 0 \\
500^{\circ} 0 \\
000^{\circ} 0 \\
000^{\circ} 0\end{array}$ & $\begin{array}{l}910^{\circ} 0 \\
510^{\circ} 0 \\
100^{\circ} 0 \\
520^{\circ} 0 \\
010^{\circ}\end{array}$ & $\begin{array}{l}020^{\circ} 0 \\
810^{\circ} 0 \\
200^{\circ} 0 \\
900^{\circ} 0 \\
210^{\circ} 0\end{array}$ \\
\hline $\begin{array}{l}500^{\circ} 0 \\
000^{\circ} \\
500^{\circ} \\
000^{\circ} 0 \\
000^{\circ} 0\end{array}$ & $\begin{array}{l}910^{\circ} 0 \\
510^{\circ} 0 \\
100^{\circ} 0 \\
520^{\circ} 0 \\
010^{\circ} 0^{-}\end{array}$ & $\begin{array}{l}020^{\circ} 0 \\
810^{\circ} 0 \\
200 \% \\
900^{\circ} 0 \\
210^{\circ} 0\end{array}$ \\
\hline $\begin{array}{l}100^{\circ} 0 \\
000^{\circ} 0 \\
100^{\circ} 0 \\
000^{\circ} 0 \\
000^{\circ} 0\end{array}$ & $\begin{array}{l}810^{\circ} 0 \\
810^{\circ} 0 \\
000^{\circ} 0 \\
810^{\circ} 0 \\
000^{-} 0\end{array}$ & $\begin{array}{l}500^{\circ} 0 \\
700^{\circ} 0 \\
100 \cdot 0 \\
000^{\circ} 0 \\
700^{\circ} 0\end{array}$ \\
\hline $\begin{array}{l}000^{\circ} 0 \\
000^{\circ} 0 \\
000^{\circ} \\
000^{\circ} 0 \\
000^{\circ} 0\end{array}$ & $\begin{array}{l}010^{*}= \\
010^{\circ} 0 \\
000^{-} 0 \\
010^{-0} 0 \\
000^{\circ} 0\end{array}$ & $\begin{array}{l}100.0 \\
100^{\circ} 0 \\
000^{\circ} 0 \\
000^{\circ} 0 \\
100^{\circ} 0\end{array}$ \\
\hline $\begin{array}{l}000^{\circ} 0 \\
000^{\circ} 0 \\
000^{\circ} 0 \\
000^{\circ} 0 \\
000^{\circ} 0\end{array}$ & $\begin{array}{l}000^{*} 0 \\
000^{\circ} 0 \\
000^{-} 0 \\
000^{\circ} 0 \\
000^{\circ} 0\end{array}$ & $\begin{array}{l}000^{\circ} \cdot 0 \\
000^{\circ} 0 \\
000^{\circ} \cdot 0 \\
000^{\circ} \cdot 0 \\
000^{\circ} 0^{\circ}\end{array}$ \\
\hline
\end{tabular}

$7 \forall 101$
$11+49$

id

d9

$7 \forall 101$
$11+89$

li

$7 \forall 101$
$1+49$

Iy

$7 \forall 101$
$11+89$

is

$7 \forall 101$

$1+89$

$7 \forall 101$

$1+89$

ly

$7 \forall 101$
$11+49$

$1+49$
14

yD 008

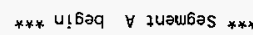

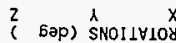




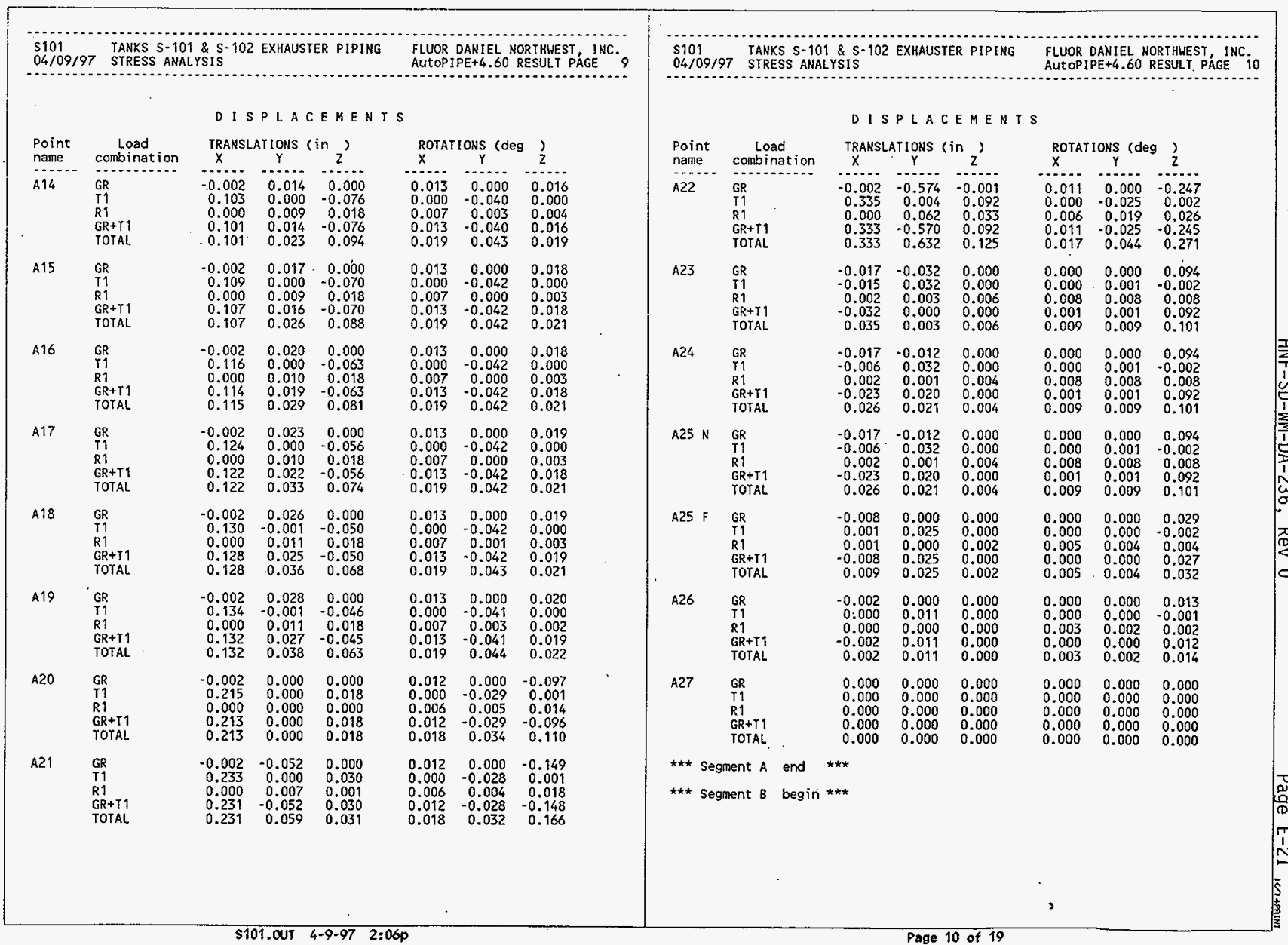




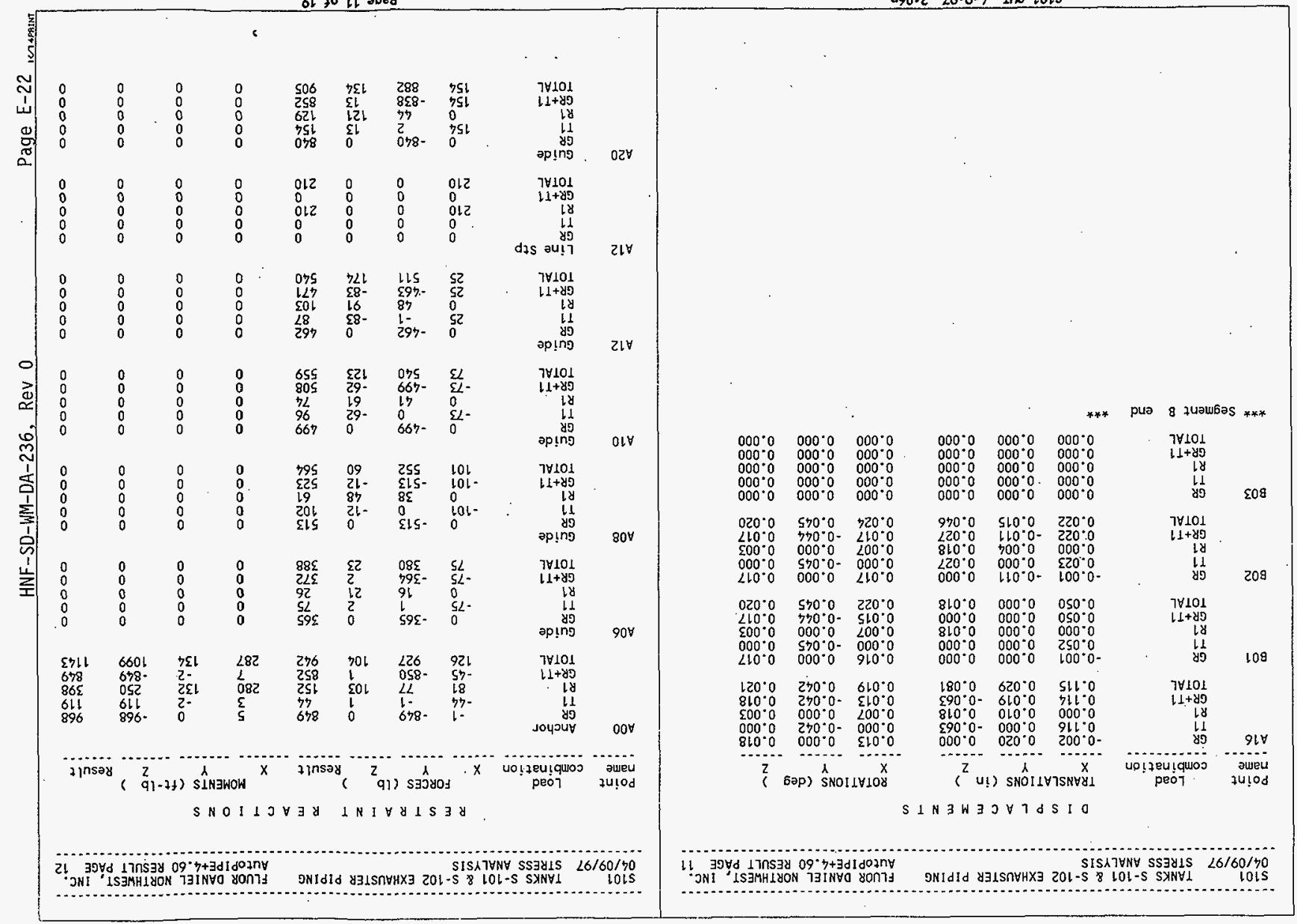




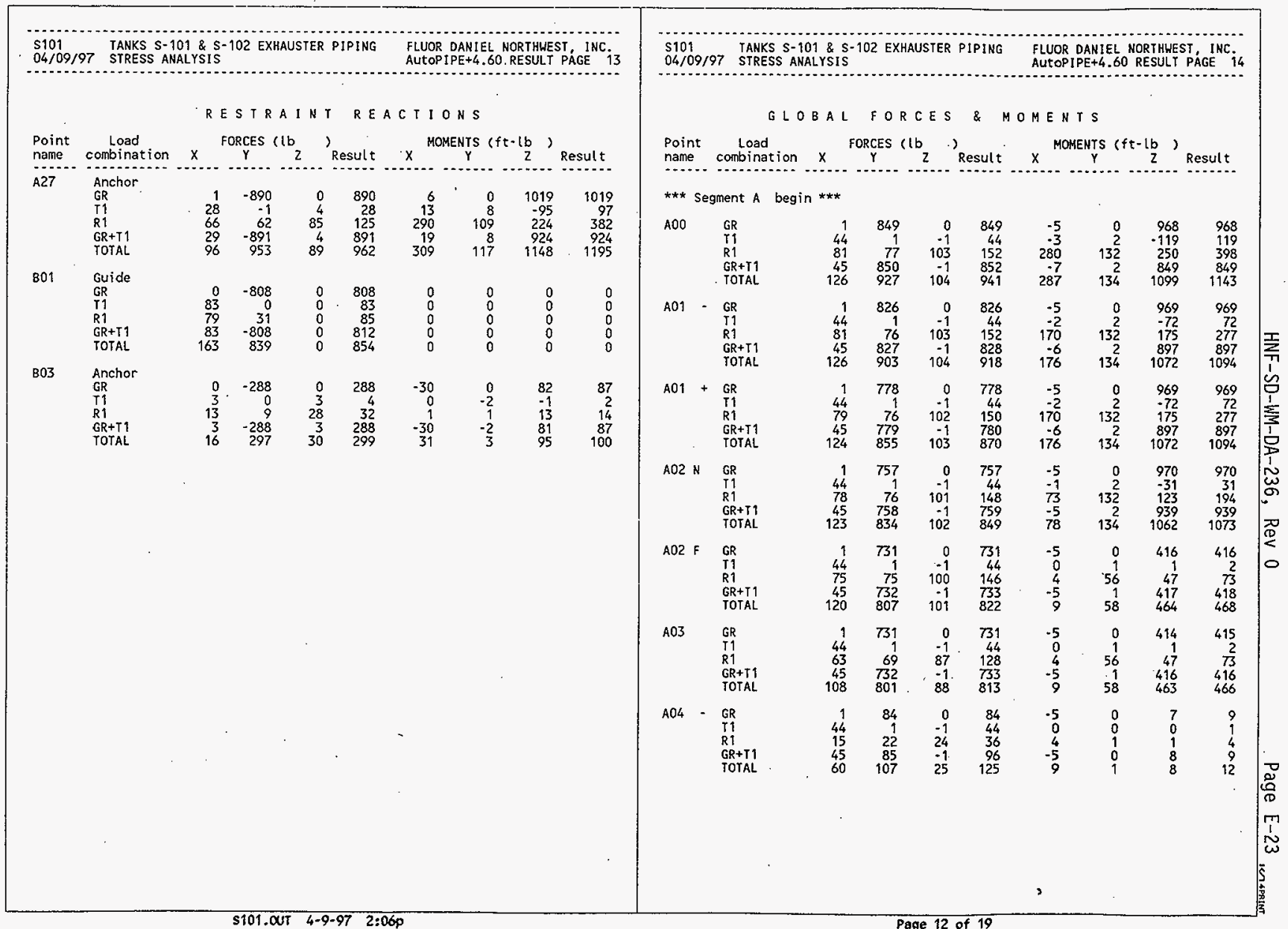




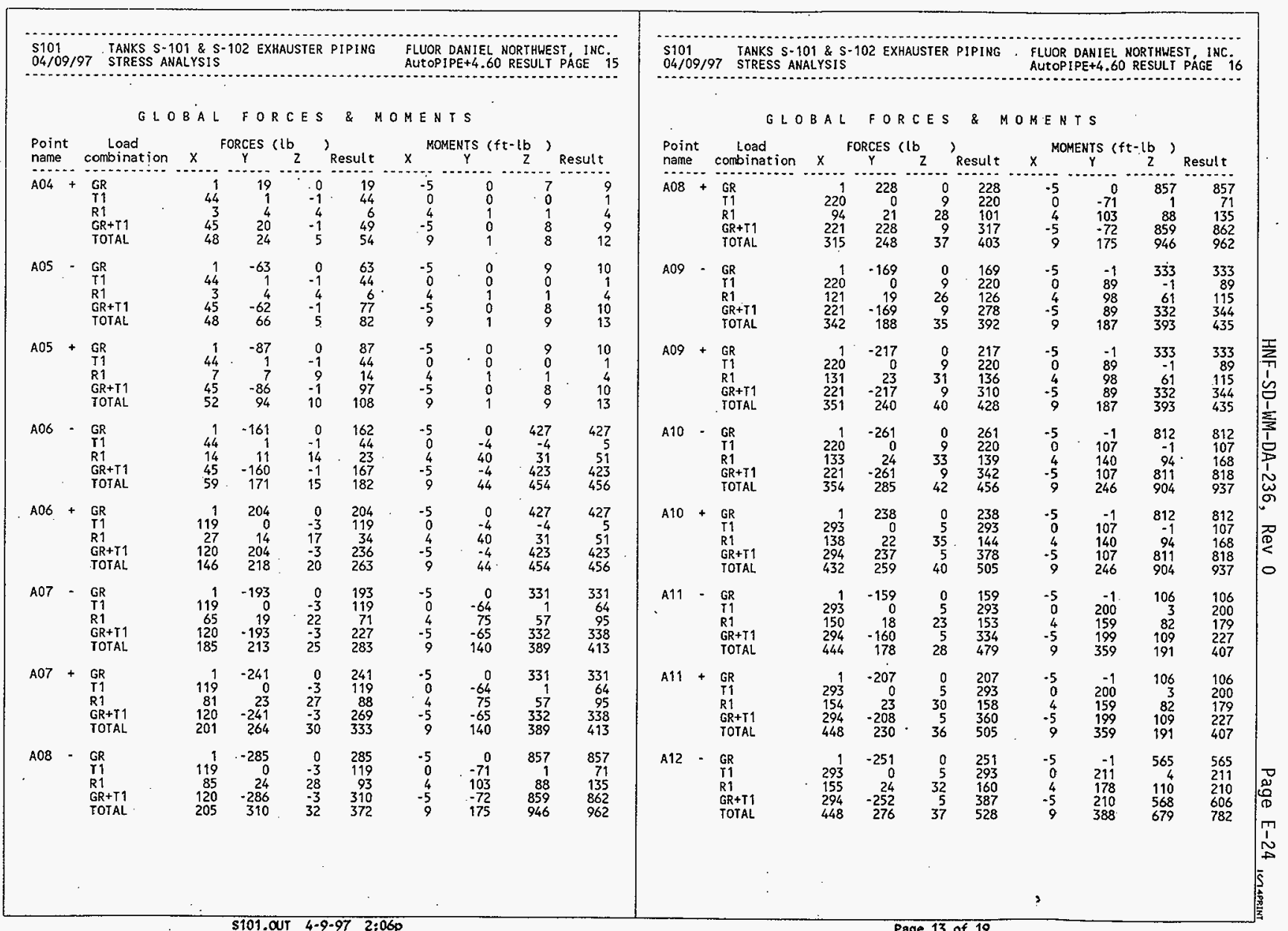




\begin{tabular}{|c|c|c|c|c|c|c|c|c|c|c|c|c|c|c|c|c|c|c|c|c|}
\hline $\begin{array}{l}\mathbf{s} 101 \\
04 / 09 / 97\end{array}$ & \multicolumn{5}{|c|}{$\begin{array}{l}\text { TANKS S-101 \& S-102 EXHAUSTER PIPING } \\
97 \text { STRESS ANALYSIS }\end{array}$} & \multicolumn{4}{|c|}{$\begin{array}{l}\text { FLUUR DANIEL NORTHLEST, INC. } \\
\text { AUTOP IPE+4.60 RESULT PAGE i7 }\end{array}$} & \multicolumn{6}{|c|}{$\begin{array}{l}\text { S101 } \\
04 / 09 / 97 \text { TANKS } s-101 \text { STRESS ANALYSIS }\end{array}$} & \multicolumn{4}{|c|}{$\begin{array}{l}\text { FLUOR DANIEL NORTHWEST, INC } \\
\text { AUTOPIPE }+4.60 \text { RESULT PAGE }\end{array}$} & \\
\hline \multicolumn{10}{|c|}{ GLOBAL FORCES \& MOMENTS } & \multicolumn{10}{|c|}{ GLOBAL FORCES \& MOMENTS } & \\
\hline $\begin{array}{l}\text { Point } \\
\text { name }\end{array}$ & $\begin{array}{l}\text { Load } \\
\text { combination }\end{array}$ & $x$ & $\underset{Y}{\operatorname{RCES}}(1$ & $j_{R}$ & Result & $x^{\text {MOM }}$ & $\underset{Y}{\text { NIS }}(f t-$ & $\stackrel{\text { Ib }}{2})^{\prime}$ & Result & $\begin{array}{l}\text { Point } \\
\text { name }\end{array}$ & $\begin{array}{l}\text { Load } \\
\text { combination }\end{array}$ & $x$ & RCES & 2 & Result & $x^{\text {MOM }}$ & $\mathrm{NTS}_{Y}<\mathrm{ft}$ & $\left.\begin{array}{rl}-(b) \\
z\end{array}\right) R$ & Result & \\
\hline A12 & $\begin{array}{l}\text { GR } \\
+\quad \text { T1 } \\
\text { R1 } \\
\text { GR+T1 } \\
\text { TOTAL }\end{array}$ & $\begin{array}{l}1 \\
268 \\
107 \\
269 \\
376\end{array}$ & $\begin{array}{r}210 \\
1 \\
32 \\
211 \\
243\end{array}$ & $\begin{array}{r}0 \\
19 \\
80 \\
19 \\
99\end{array}$ & $\begin{array}{l}210 \\
269 \\
137 \\
343 \\
459\end{array}$ & $\begin{array}{r}-5 \\
0 \\
4 \\
-5 \\
9\end{array}$ & $\begin{array}{l}-11 \\
211 \\
178 \\
210 \\
388\end{array}$ & $\begin{array}{r}565 \\
4 \\
110 \\
568 \\
679\end{array}$ & $\begin{array}{l}565 \\
211 \\
210 \\
606 \\
782\end{array}$ & A18 & $\begin{array}{l}\text { GR } \\
\text { I1 } \\
\text { R1 } \\
\text { GR+T1 } \\
\text { TOTAL }\end{array}$ & $\begin{array}{r}1 \\
182 \\
64 \\
183 \\
247\end{array}$ & $\begin{array}{r}-177 \\
1 \\
17 \\
-176 \\
192\end{array}$ & $\begin{array}{r}0 \\
17 \\
65 \\
17 \\
82\end{array}$ & $\begin{array}{r}177 \\
182 \\
93 \\
254 \\
323\end{array}$ & $\begin{array}{r}6 \\
0 \\
5 \\
6 \\
11\end{array}$ & $\begin{array}{r}1 \\
-211 \\
407 \\
-210 \\
617\end{array}$ & $\begin{array}{r}-199 \\
-6 \\
159 \\
-205 \\
363\end{array}$ & $\begin{array}{l}199 \\
211 \\
437 \\
293 \\
716\end{array}$ & \\
\hline$A 13$ & $\begin{array}{l}\text { GR } \\
\text { T1 } \\
\text { R1 } \\
\text { GR+T1 } \\
\text { TOTAL }\end{array}$ & $\begin{array}{r}1 \\
268 \\
95 \\
269 \\
364\end{array}$ & $\begin{array}{r}56 \\
1 \\
29 \\
57 \\
86\end{array}$ & $\begin{array}{r}0 \\
19 \\
72 \\
19 \\
91\end{array}$ & $\begin{array}{l}56 \\
269 \\
123 \\
276 \\
385\end{array}$ & $\begin{array}{r}-5 \\
0 \\
4 \\
-5 \\
9\end{array}$ & $\begin{array}{l}-1 \\
343 \\
372 \\
342 \\
715\end{array}$ & $\begin{array}{r}-366 \\
-3 \\
120 \\
-369 \\
490\end{array}$ & $\begin{array}{l}367 \\
343 \\
391 \\
504 \\
866\end{array}$ & $\mathrm{~A} 19$ & $\begin{array}{l}\text { GR } \\
I 1 \\
R 1 \\
\text { GR+T1 } \\
\text { IOTAL }\end{array}$ & $\begin{array}{r}1 \\
182 \\
63 \\
183 \\
246\end{array}$ & $\begin{array}{r}-188 \\
1 \\
17 \\
-187 \\
204\end{array}$ & $\begin{array}{r}0 \\
17 \\
66 \\
17 \\
83\end{array}$ & $\begin{array}{r}188 \\
182 \\
93 \\
262 \\
330\end{array}$ & $\begin{array}{r}6 \\
0 \\
5 \\
6 \\
11\end{array}$ & $\begin{array}{r}1 \\
-203 \\
374 \\
-202 \\
576\end{array}$ & $\begin{array}{r}-108 \\
-6 \\
160 \\
-114 \\
274\end{array}$ & $\begin{array}{l}108 \\
203 \\
407 \\
232 \\
638\end{array}$ & \\
\hline A13 & $\begin{array}{l}\mathrm{GR} \\
\mathrm{TI} \\
\mathrm{RI} \\
\mathrm{GR+T1} \\
\text { TOTAL }\end{array}$ & $\begin{array}{r}1 \\
268 \\
87 \\
269 \\
356\end{array}$ & $\begin{array}{r}8 \\
1 \\
25 \\
9 \\
34\end{array}$ & $\begin{array}{r}0 \\
19 \\
63 \\
19 \\
81\end{array}$ & $\begin{array}{r}8 \\
269 \\
110 \\
270 \\
367\end{array}$ & $\begin{array}{r}-5 \\
0 \\
4 \\
-5 \\
9\end{array}$ & $\begin{array}{l}-1 \\
343 \\
372 \\
342 \\
715\end{array}$ & $\begin{array}{r}-366 \\
-3 \\
120 \\
-369 \\
490\end{array}$ & $\begin{array}{l}367 \\
343 \\
391 \\
504 \\
866\end{array}$ & A19 + & $\begin{array}{l}\text { + } G R \\
I 1 \\
R 1 \\
\text { GR+T1 } \\
\text { TOTAL }\end{array}$ & $\begin{array}{r}1 \\
182 \\
54 \\
183 \\
237\end{array}$ & $\begin{array}{r}-236 \\
1 \\
21 \\
-235 \\
255\end{array}$ & $\begin{array}{r}0 \\
17 \\
77 \\
17 \\
94\end{array}$ & $\begin{array}{l}236 \\
182 \\
96 \\
298 \\
361\end{array}$ & $\begin{array}{r}6 \\
0 \\
5 \\
6 \\
11\end{array}$ & $\begin{array}{r}1 \\
-203 \\
374 \\
-202 \\
576\end{array}$ & $\begin{array}{r}-108 \\
-6 \\
160 \\
-114 \\
274\end{array}$ & $\begin{array}{l}108 \\
203 \\
407 \\
232 \\
638\end{array}$ & 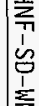 \\
\hline A14 & $\begin{array}{l}\text { GR } \\
\mathrm{T} 1 \\
\mathrm{R} 1 \\
\text { GR+I1 } \\
\text { TOIAL }\end{array}$ & $\begin{array}{r}1 \\
268 \\
86 \\
269 \\
355\end{array}$ & $\begin{array}{r}-3 \\
1 \\
24 \\
-2 \\
26\end{array}$ & $\begin{array}{r}0 \\
19 \\
61 \\
19 \\
80\end{array}$ & $\begin{array}{l}3 \\
269 \\
108 \\
270 \\
365\end{array}$ & $\begin{array}{r}-5 \\
0 \\
4 \\
-5 \\
9\end{array}$ & $\begin{array}{l}-1 \\
353 \\
403 \\
352 \\
754\end{array}$ & $\begin{array}{r}-368 \\
-3 \\
131 \\
-371 \\
502\end{array}$ & $\begin{array}{l}368 \\
353 \\
423 \\
511 \\
906\end{array}$ & A20 & $\begin{array}{ll}- & G R \\
T 1 \\
R I \\
\text { GR+T1 } \\
\text { TOTAL }\end{array}$ & $\begin{array}{r}1 \\
182 \\
41 \\
183 \\
224\end{array}$ & $\begin{array}{r}-434 \\
1 \\
25 \\
-433 \\
458\end{array}$ & $\begin{array}{r}0 \\
17 \\
86 \\
17 \\
103\end{array}$ & $\begin{array}{l}434 \\
182 \\
99 \\
470 \\
520\end{array}$ & $\begin{array}{r}6 \\
0 \\
5 \\
6 \\
11\end{array}$ & $\begin{array}{r}1 \\
-52 \\
367 \\
-52 \\
419\end{array}$ & $\begin{array}{r}2906 \\
-15 \\
226 \\
2890 \\
3116\end{array}$ & $\begin{array}{r}2906 \\
55 \\
431 \\
2891 \\
3144\end{array}$ & $\begin{array}{l}3 \\
1 \\
. \\
1 \\
1 \\
w \\
w \\
o\end{array}$ \\
\hline A15 & $\begin{array}{l}\text { GR } \\
\text { T1 } \\
\text { R1 } \\
\text { GR+r1 } \\
\text { TOTAL }\end{array}$ & $\begin{array}{r}1 \\
268 \\
83 \\
269 \\
353\end{array}$ & $\begin{array}{r}-21 \\
1 \\
23 \\
-20 \\
43\end{array}$ & $\begin{array}{r}0 \\
19 \\
58 \\
19 \\
77\end{array}$ & $\begin{array}{l}21 \\
269 \\
104 \\
271 \\
363\end{array}$ & $\begin{array}{r}-5 \\
0 \\
4 \\
-5 \\
9\end{array}$ & $\begin{array}{l}-1 \\
365 \\
441 \\
364 \\
806\end{array}$ & $\begin{array}{r}-360 \\
-4 \\
145 \\
-364 \\
510\end{array}$ & $\begin{array}{l}360 \\
365 \\
465 \\
515 \\
953\end{array}$ & A20 & $\begin{array}{l}+\mathrm{GR} \\
\mathrm{T} I \\
\mathrm{R} 1 \\
\mathrm{GR}+\mathrm{T1} \\
\text { TOTAL }\end{array}$ & $\begin{array}{l}1 \\
28 \\
37 \\
29 \\
66\end{array}$ & $\begin{array}{r}406 \\
-1 \\
24 \\
405 \\
429\end{array}$ & $\begin{array}{r}0 \\
4 \\
36 \\
4 \\
40\end{array}$ & $\begin{array}{r}406 \\
28 \\
57 \\
406 \\
436\end{array}$ & $\begin{array}{r}6 \\
0 \\
5 \\
6 \\
11\end{array}$ & $\begin{array}{r}1 \\
-52 \\
367 \\
-52 \\
419\end{array}$ & $\begin{array}{r}2906 \\
-15 \\
226 \\
2890 \\
3116\end{array}$ & $\begin{array}{r}2906 \\
55 \\
431 \\
2891 \\
3144\end{array}$ & \\
\hline A16 & $\begin{array}{l}-\quad \text { GR } \\
T 1 \\
\text { R1 } \\
\text { GR+r1 } \\
\text { TOTAL }\end{array}$ & $\begin{array}{r}1 \\
268 \\
80 \\
269 \\
349\end{array}$ & $\begin{array}{r}-67 \\
1 \\
21 \\
-66 \\
88\end{array}$ & $\begin{array}{c}0 \\
19 \\
54 \\
19 \\
73\end{array}$ & $\begin{array}{l}67 \\
269 \\
99 \\
278 \\
367\end{array}$ & $\begin{array}{r}-5 \\
0 \\
4 \\
-5 \\
9\end{array}$ & $\begin{array}{l}-1 \\
381 \\
487 \\
380 \\
866\end{array}$ & $\begin{array}{r}-324 \\
-5 \\
161 \\
-328 \\
490\end{array}$ & $\begin{array}{l}324 \\
381 \\
513 \\
502 \\
995\end{array}$ & A21 & $\begin{array}{l}\text { - } G R \\
T 1 \\
R 1 \\
R 1 \\
\text { GR+T1 } \\
\text { TOTAL }\end{array}$ & $\begin{array}{l}1 \\
28 \\
34 \\
29 \\
63\end{array}$ & $\begin{array}{r}362 \\
-1 \\
24 \\
361 \\
384\end{array}$ & $\begin{array}{r}0 \\
4 \\
35 \\
4 \\
39\end{array}$ & $\begin{array}{r}362 \\
28 \\
54 \\
362 \\
391\end{array}$ & $\begin{array}{r}8 \\
0 \\
5 \\
6 \\
11\end{array}$ & $\begin{array}{r}0 \\
-45 \\
301 \\
-44 \\
346\end{array}$ & $\begin{array}{r}2138 \\
-13 \\
181 \\
2125 \\
2306\end{array}$ & $\begin{array}{r}2138 \\
47 \\
351 \\
2126 \\
2332\end{array}$ & \\
\hline A16 + & $\begin{array}{l}\text { GR } \\
T 1 \\
\text { R1 } \\
\text { GR+T1 } \\
\text { TOTAL }\end{array}$ & $\begin{array}{r}1 \\
182 \\
72 \\
183 \\
255\end{array}$ & $\begin{array}{r}-98 \\
1 \\
17 \\
-97 \\
113\end{array}$ & $\begin{array}{l}0 \\
17 \\
55 \\
17 \\
72\end{array}$ & $\begin{array}{r}98 \\
182 \\
92 \\
208 \\
288\end{array}$ & $\begin{array}{r}6 \\
0 \\
5 \\
6 \\
11\end{array}$ & $\begin{array}{r}1 \\
-236 \\
495 \\
-235 \\
730\end{array}$ & $\begin{array}{r}-406 \\
+4 \\
152 \\
-410 \\
561\end{array}$ & $\begin{array}{l}406 \\
236 \\
518 \\
472 \\
921\end{array}$ & A21 & $\begin{array}{l}+\quad \text { GR } \\
\text { T1 } \\
\text { R1 } \\
\text { GR+T1 } \\
\text { TOTAL }\end{array}$ & $\begin{array}{l}1 \\
28 \\
24 \\
29 \\
53\end{array}$ & $\begin{array}{r}314 \\
-1 \\
22 \\
313 \\
334\end{array}$ & $\begin{array}{r}0 \\
4 \\
33 \\
4 \\
37\end{array}$ & $\begin{array}{r}314 \\
28 \\
47 \\
314 \\
341\end{array}$ & $\begin{array}{r}8 \\
0 \\
5 \\
6 \\
11\end{array}$ & $\begin{array}{r}0 \\
-45 \\
301 \\
-44 \\
346\end{array}$ & $\begin{array}{r}2138 \\
-13 \\
181 \\
2185 \\
2306\end{array}$ & $\begin{array}{r}2138 \\
47 \\
351 \\
2126 \\
2332\end{array}$ & \\
\hline A17 & $\begin{array}{l}\text { GR } \\
T 1 \\
R 1 \\
\text { GR+T } \\
\text { TOTAL }\end{array}$ & $\begin{array}{r}1 \\
182 \\
67 \\
183 \\
250\end{array}$ & $\begin{array}{r}-144 \\
1 \\
17 \\
-143 \\
159\end{array}$ & $\begin{array}{r}0 \\
17 \\
61 \\
17 \\
78\end{array}$ & $\begin{array}{r}144 \\
182 \\
92 \\
233 \\
306\end{array}$ & $\begin{array}{r}6 \\
0 \\
5 \\
6 \\
11\end{array}$ & $\begin{array}{r}1 \\
-222 \\
448 \\
-221 \\
669\end{array}$ & $\begin{array}{r}-306 \\
-5 \\
156 \\
-310 \\
466\end{array}$ & $\begin{array}{l}306 \\
222 \\
474 \\
381 \\
816\end{array}$ & A22 & $\begin{array}{l}\mathrm{GR} \\
\mathrm{T} 1 \\
\mathrm{R1} \\
\mathrm{GR}+\mathrm{T} 1 \\
\text { TOTAL }\end{array}$ & $\begin{array}{r}1 \\
28 \\
8 \\
29 \\
38\end{array}$ & $\begin{array}{l}63 \\
-1 \\
10 \\
62 \\
72\end{array}$ & $\begin{array}{r}0 \\
4 \\
19 \\
4 \\
23\end{array}$ & $\begin{array}{l}63 \\
28 \\
23 \\
69 \\
85\end{array}$ & $\begin{array}{r}6 \\
0 \\
5 \\
5 \\
6 \\
11\end{array}$ & $\begin{array}{r}0 \\
-2 \\
2 \\
-2 \\
3\end{array}$ & $\begin{array}{r}-2 \\
0 \\
1 \\
-3 \\
4\end{array}$ & $\begin{array}{r}7 \\
2 \\
6 \\
7 \\
12\end{array}$ & 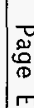 \\
\hline & & & & & & . & & & & & & & & & & , & & & & \\
\hline
\end{tabular}


pua g дuəแ6อง ***

\begin{tabular}{|c|c|c|c|c|}
\hline $\begin{array}{l}001 \\
28 \\
71 \\
2 \\
28\end{array}$ & $\begin{array}{l}56 \\
18 \\
51 \\
1- \\
28\end{array}$ & $\begin{array}{l}\varepsilon \\
z- \\
1 \\
z- \\
0\end{array}$ & $\begin{array}{l}\boldsymbol{l} \varepsilon \\
0 \varepsilon- \\
l \\
0 \\
0 \varepsilon-\end{array}$ & $\begin{array}{l}712 \\
802 \\
92 \\
7 \\
802\end{array}$ \\
\hline $\begin{array}{l}66 \\
98 \\
71 \\
2 \\
28\end{array}$ & $\begin{array}{l}56 \\
18 \\
51 \\
1- \\
28\end{array}$ & $\begin{array}{l}2 \\
l \\
l \\
l \\
0 .\end{array}$ & $\begin{array}{l}62 \\
82- \\
1 \\
0 \\
82-\end{array}$ & $\begin{array}{l}92 \\
29 \\
52 \\
+5 \\
99\end{array}$ \\
\hline $\begin{array}{l}66 \\
98 \\
71 \\
2 \\
28\end{array}$ & $\begin{array}{l}56 \\
18 \\
51 \\
1- \\
28\end{array}$ & $\begin{array}{l}z \\
l \\
l \\
l \\
0\end{array}$ & $\begin{array}{l}62 \\
82- \\
b \\
0 \\
82 .\end{array}$ & $\begin{array}{l}862 \\
\sum 82 \\
\sum 7 \\
7 \\
\sum 82\end{array}$ \\
\hline $\begin{array}{l}1021 \\
2511 \\
701 \\
11 \\
2511\end{array}$ & $\begin{array}{l}56 \\
18 \\
\varepsilon 1 \\
1- \\
28\end{array}$ & $\begin{array}{l}301 \\
01 \\
76 \\
11 \\
1-\end{array}$ & $\begin{array}{l}\sum 6 l l \\
671 L- \\
\sum 7 \\
0 \\
6711-\end{array}$ & $\begin{array}{l}\sum\langle\hbar \\
2 S t \\
79 \\
\eta \\
2 S t\end{array}$ \\
\hline $\begin{array}{l}1021 \\
2511 \\
501 \\
111 \\
2511\end{array}$ & $\begin{array}{l}56 \\
18 \\
\varepsilon 1 \\
1- \\
28\end{array}$ & $\begin{array}{l}706 \\
06 \\
76 \\
61 \\
b-\end{array}$ & $\begin{array}{l}\varepsilon 611 \\
6711- \\
\varepsilon \% \\
0 \\
6711 .\end{array}$ & $\begin{array}{l}165 \\
995 \\
69 \\
28 \\
958\end{array}$ \\
\hline $\begin{array}{l}002 \\
029 \\
18 \\
219 \\
88\end{array}$ & $\begin{array}{l}56 \\
18 \\
\varepsilon 1 \\
1- \\
28\end{array}$ & $\begin{array}{l}769 \\
519 \\
62 \\
219 \\
2 .\end{array}$ & $\begin{array}{l}6 l \\
11- \\
8 \\
1 \\
11-\end{array}$ & $\begin{array}{l}571 \\
26 \\
96 \\
28 \\
15\end{array}$ \\
\hline
\end{tabular}

\begin{tabular}{|c|c|}
\hline $\begin{array}{l}212 \\
802- \\
5 \\
0 \\
802-\end{array}$ & $\begin{array}{l}6 \\
\xi \\
9 \\
\varepsilon \\
0\end{array}$ \\
\hline $\begin{array}{l}12 \\
99 \\
5 \\
0 \\
99\end{array}$ & $\begin{array}{l}6 \\
5 \\
9 \\
5 \\
0\end{array}$ \\
\hline $\begin{array}{l}562 \\
\sum 82 \\
16 \\
0 \\
£ 82\end{array}$ & $\sum$ \\
\hline $\begin{array}{l}697 \\
257 \\
21 \\
0 \\
25\rangle\end{array}$ & $\begin{array}{l}\xi \\
0\end{array}$ \\
\hline $\begin{array}{l}\angle 9 \varepsilon \\
9 S \Sigma- \\
\text { ZL } \\
0 \\
9 S E-\end{array}$ & 8 \\
\hline $\begin{array}{l}Z \downarrow \\
\text { lE } \\
Z 1 \\
0 \\
\text { IS }\end{array}$ & .0 \\
\hline
\end{tabular}

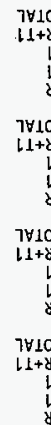

$11+89$

i.

$7 \forall 101$
$11+89$

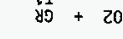

$7 \forall 101$

$11+\mathrm{y}$

ly

$7 \forall 101$
$11+89$

11
70101
$11+40$
18
11

$7 \% 10$.
$11+y 9$

$17+\frac{10}{14}$

४อ

*** U!̣วaq g quaw6as ***

*** pua $\forall$ LUaш́as ***

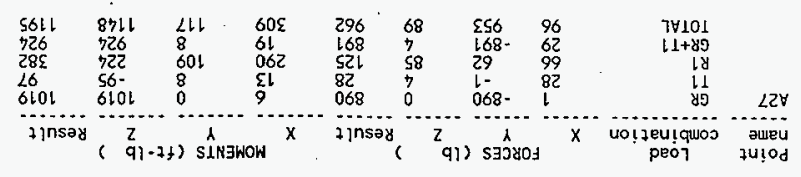

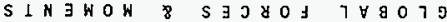




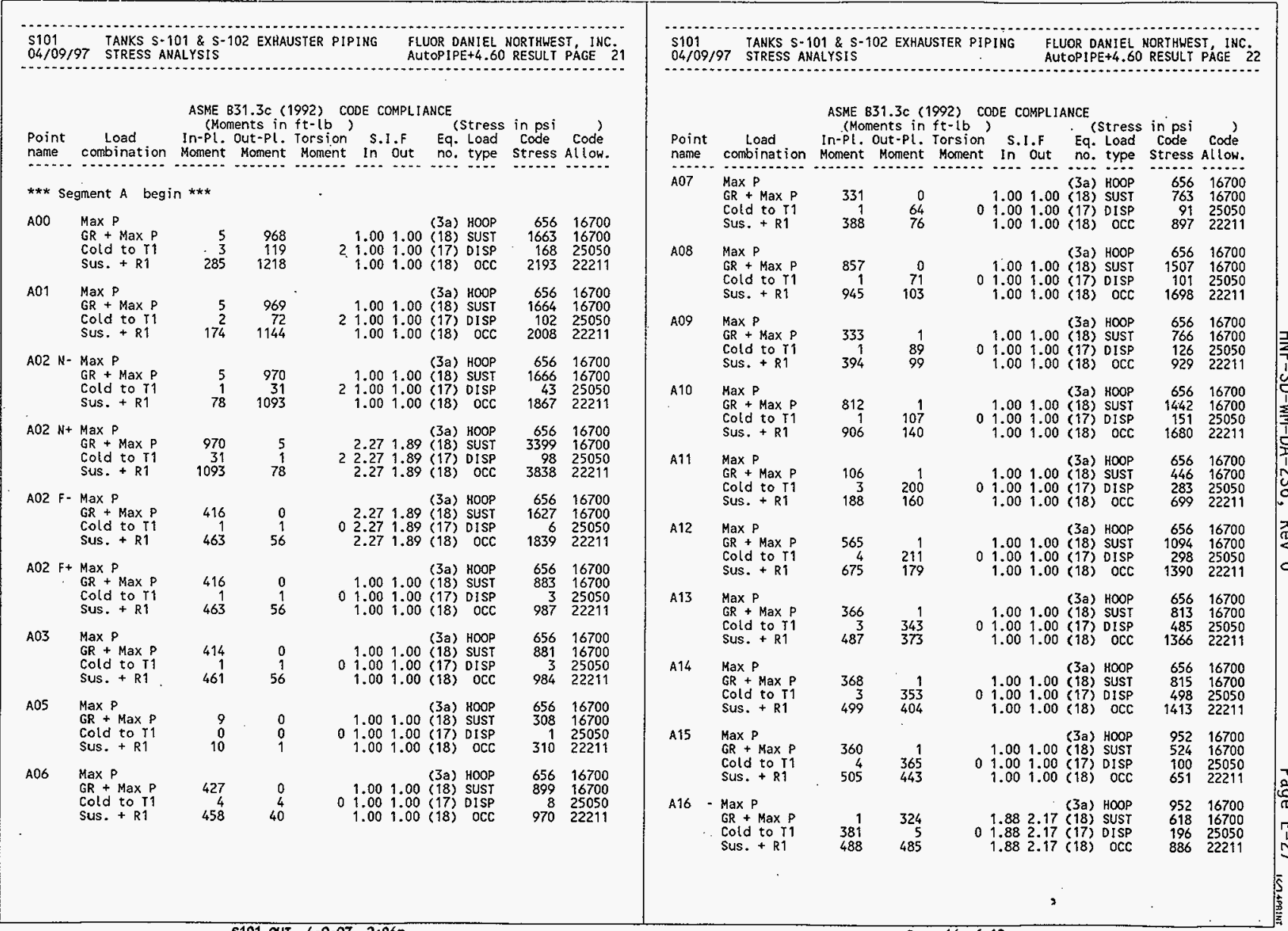




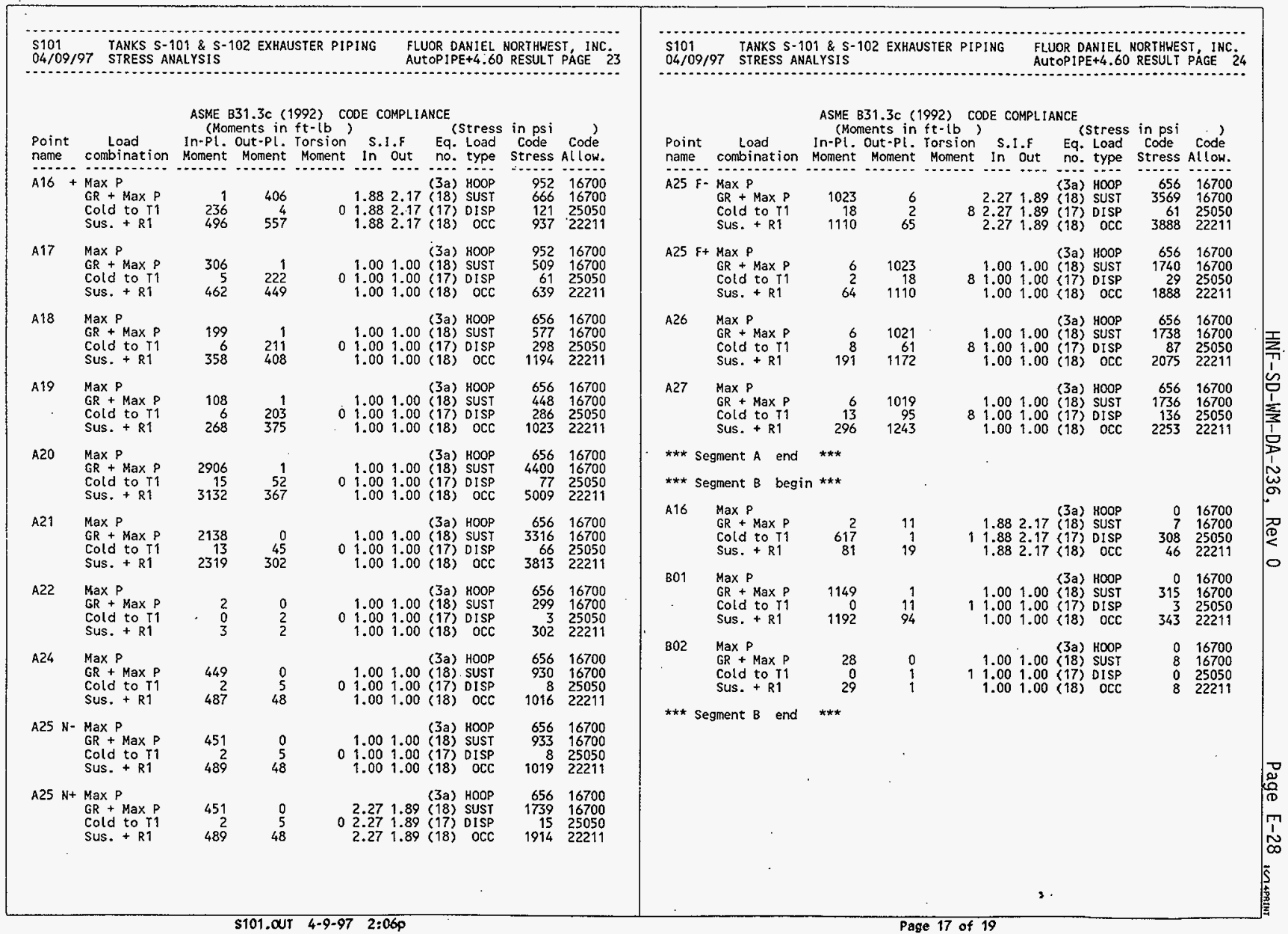




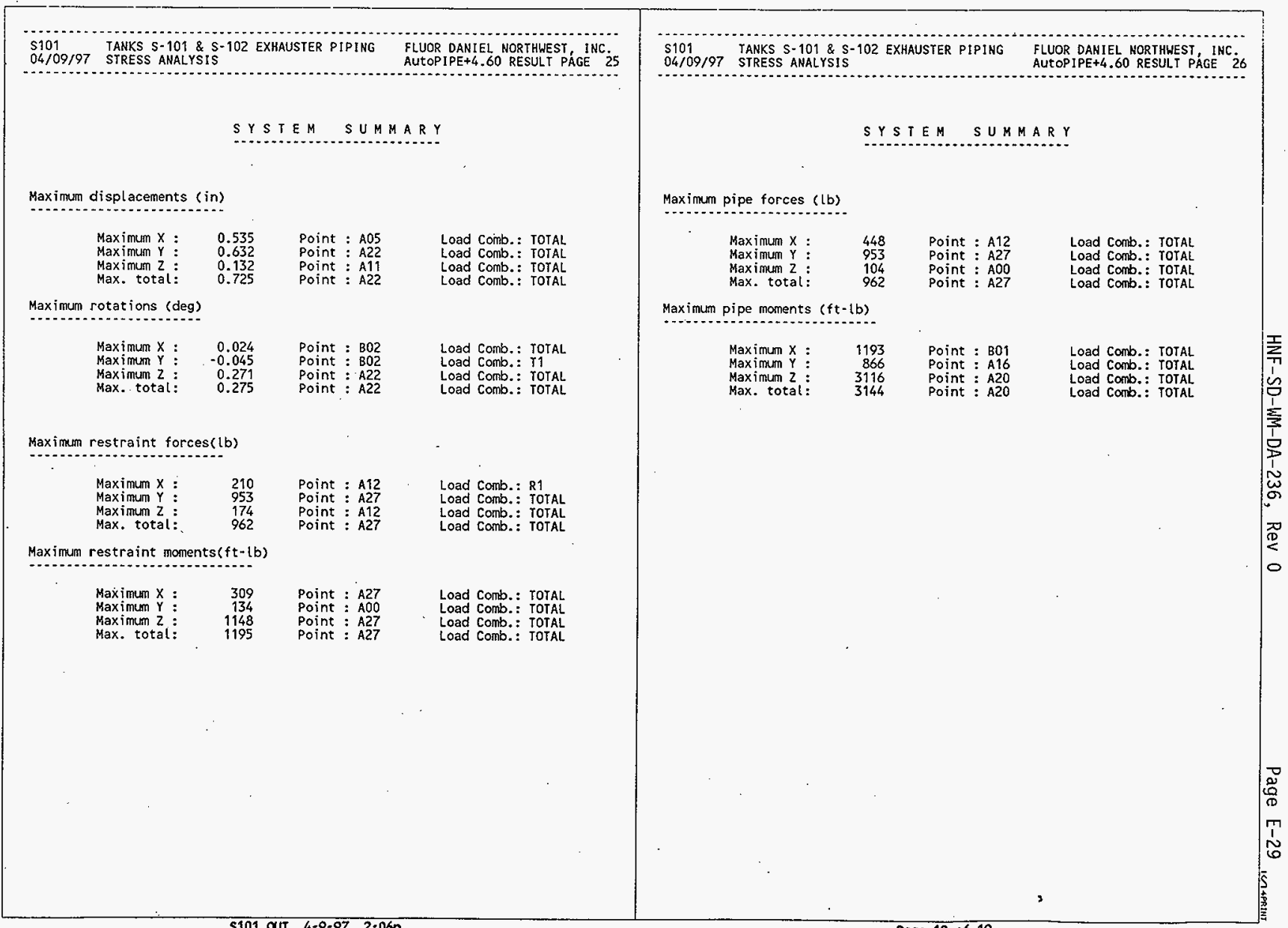




S101 TANKS S-101 \& $\mathrm{S}-102$ EXHAUSTER PIPING
$04 / 09 / 97$ STRESS ANALYSIS
SUTOPIPE+4.60 RESULT PAGE 27

\section{SYSTEM SUMMARY}

Maximum sustained stress

$$
\begin{array}{ll}
\text { Point } & \text { A20 } \\
\text { Stress psi } & 4400 \\
\text { Allowable ps } i \quad & 16700 \\
\text { Ratio } & 0.26 \\
\text { Load combination } & \text { GR }+ \text { Max P }
\end{array}
$$

Maximum displacement stress

$$
\begin{array}{ll}
\text { Point } & \text { A14 } \\
\text { Stress psi } & \vdots 498 \\
\text { Allowable psi } & \vdots 25050 \\
\text { Ratio } & \vdots 0.02 \\
\text { Load combination } & : \text { Cold to } \mathrm{r} 1 .
\end{array}
$$

Maximum occasional stress

$$
\begin{array}{ll}
\text { Point } & : \begin{array}{l}
A 20 \\
\text { Stress psi }
\end{array} \\
\text { At lowable psi } & 22211 \\
\text { Ratio } & 0.23 \\
\text { Load combination } & \text { Sus. + R1 }
\end{array}
$$

Maximum hoop stress

$$
\begin{array}{ll}
\text { Point } & \text { A15 } \\
\text { Stress psi } & : 952 \\
\text { Allowable psi } & : 16700 \\
\text { Ratio } & 0.06 \\
\text { Load combination } & \text { Max P }
\end{array}
$$

Maximum sustained stress ratio

$$
\begin{array}{ll}
\text { Point } & \text { A20 } \\
\text { Stress psi } & 4400 \\
\text { Allowable psi } & 16700 \\
\text { Ratio } & 0.26 \\
\text { Load combination } & \mathrm{GR}+\text { Max P }
\end{array}
$$

Maximum displacement stress ratio

$$
\begin{array}{ll}
\text { Point } & \text { A14 } \\
\text { Stress psi } & : 498 \\
\text { Allowable psi } & : 25050 \\
\text { Ratio } & 0.02 \\
\text { Load combination } & \text { Cold to T1 }
\end{array}
$$

S101 IANKS $S-101 \& S-102$ EXHAUSIER PIPING FLUOR DANIEL NORIHWEST INC.

AUTOPIPE+4.60 RESULT PAGE 28

(1)

\section{SYSTEM SUMMARY}

Maximum occasional stress ratio

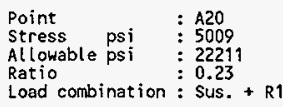

Maximum hoop stress ratio

$\begin{array}{ll}\text { Point } & \text { A15 } \\ \text { Stress psi } & 952 \\ \text { Allowable psi } & : 16700 \\ \text { Ratio } & 0.06 \\ \text { Load combination } & \text { Max P }\end{array}$

*** The system satisfies ASHE B31.3 code requirements * * * 


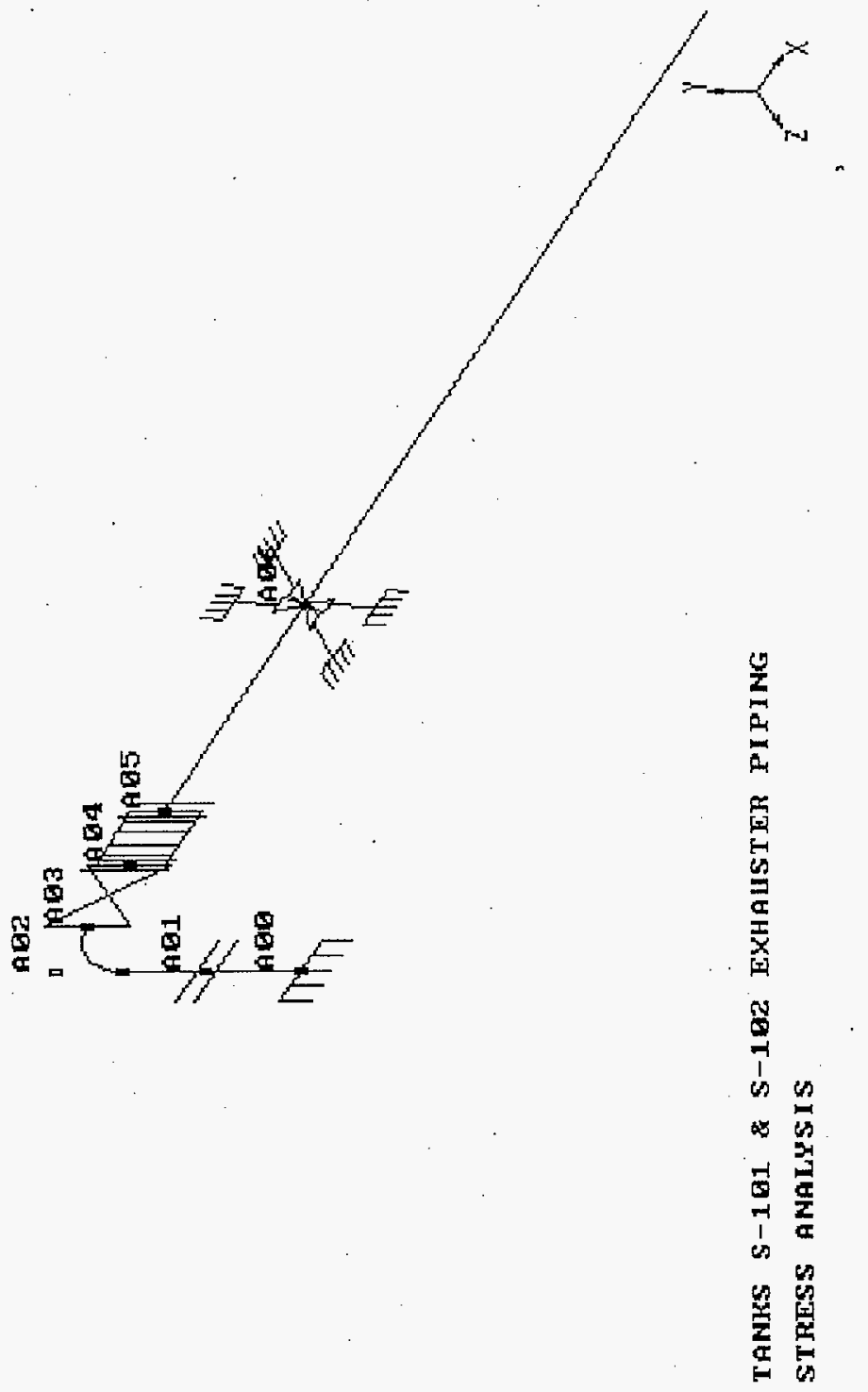




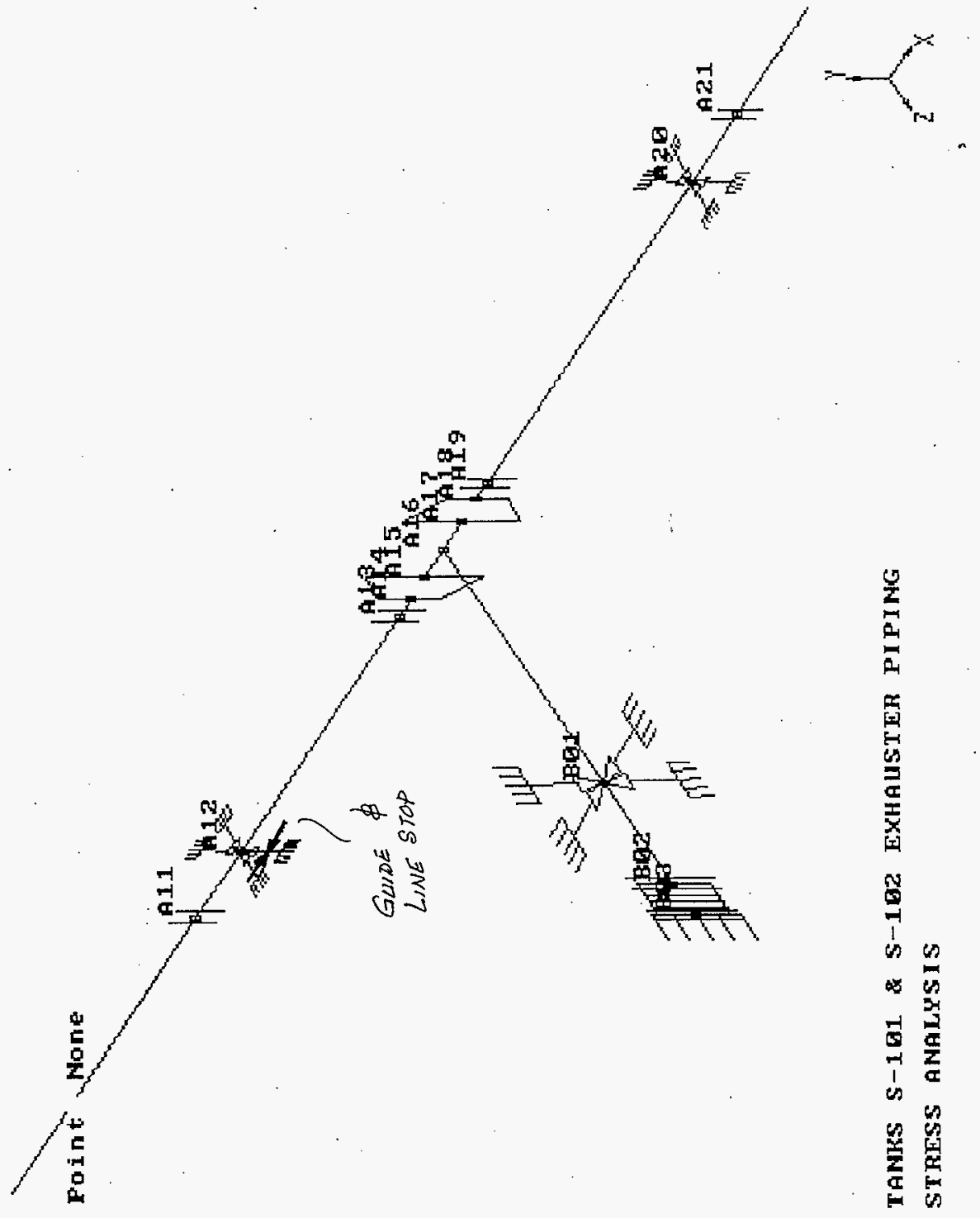




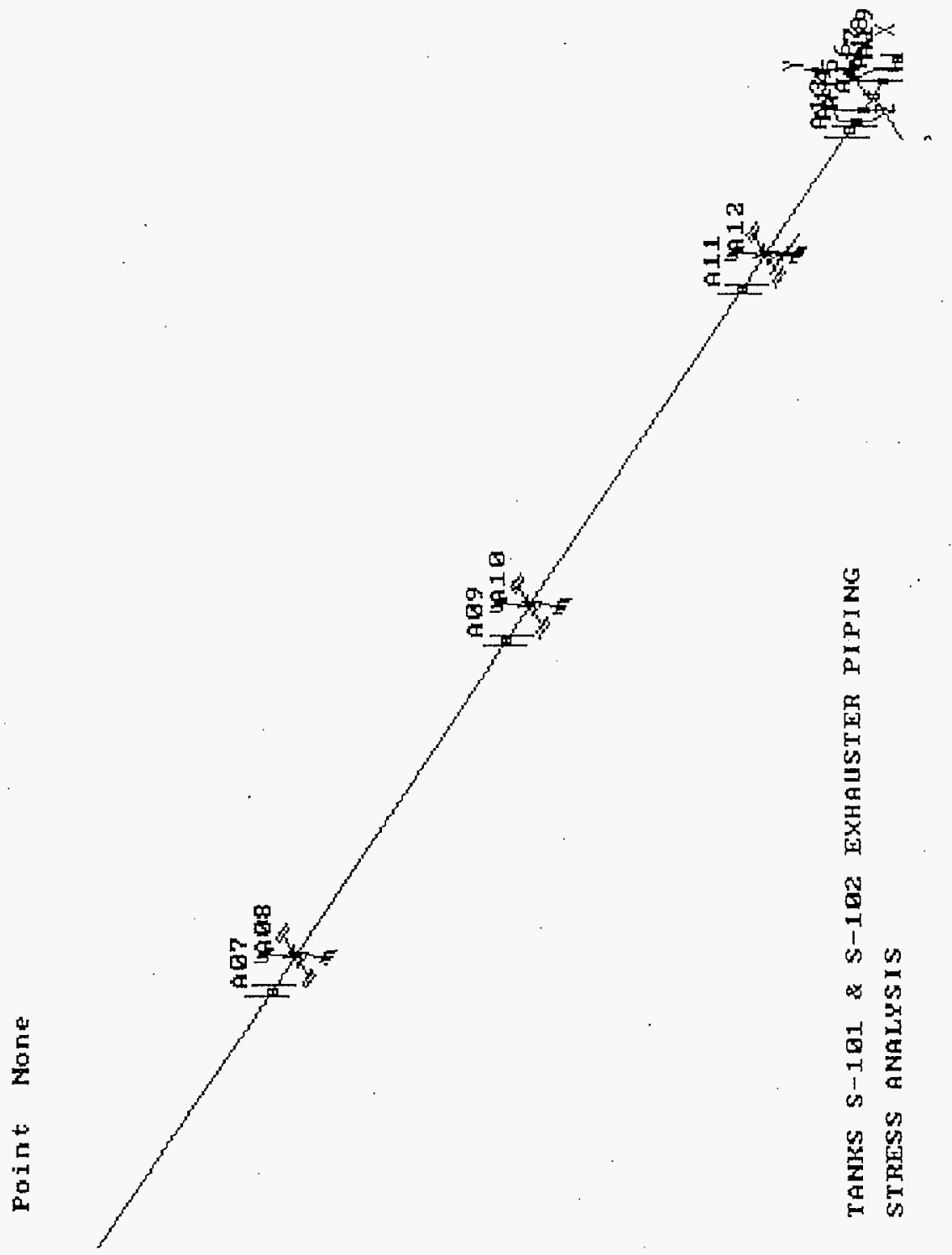



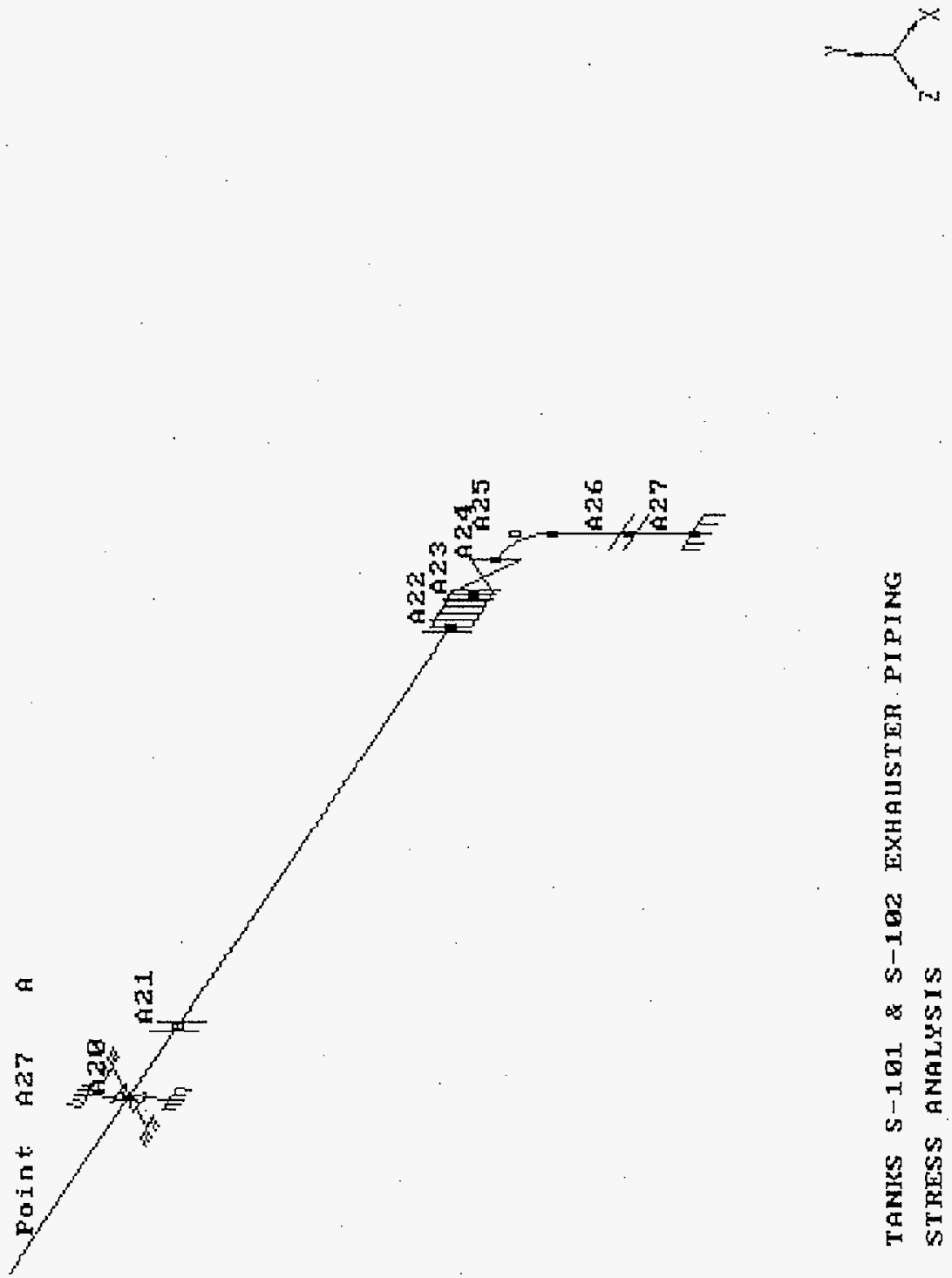
HNF-SD-WM-DA-236

Rev. 0

APPENDIX F: JUSTIFICATION FOR ELECTRICAL SUPPLY AND DISTRIBUTION 


\section{General}

This appendix documents the electrical installation design for the subject saltwell exhauster units. The onsite fabricated 500 CFM exhauster requires a 3 phase, 480 VAC, 30 ampere service. (Empirical testing of the 500 CFM unit indicates that the maximum load is less than 15 amps at $480 \mathrm{VAC}$.). The offsite fabricated $1000 \mathrm{CFM}$ unit requires a 3 phase, $480 \mathrm{VAC}, 60$ ampere service. The cable sizing calculations for each installation design are based upon this information and which exhauster unit is being utilized.

Existing power distribution equipment was employed for each of the installations. Fuse or circuit breaker size has been revised to match the individual requirements. Because the facilities being serviced are essentially de-activated (with corresponding reductions in electrical loads from active status) it was not deemed necessary to do a rigorous electrical loading analysis on each of the sources.

Where possible, existing electrical cable has been used to provide the necessary power. In locations where new cables are required, the emphasis was directed at using temporary raceway (Yellow Jacket) where appropriate or installing exposed conduit where it was viable. Buried conduit is the least economical of the options and was employed only when necessary.

All installations are to be in accordance with the current edition of the National Electrical Code. The exhauster skids are to be grounded to the nearest above-ground equipment ground and the exhaust duct will be bonded across each of the flange faces. 


\section{1-S-109 SALTWELL EXHAUSTER ELECTRICAL INSTALLATION}

1. 500. CFM exhauster at 241-S-109 (single service).

2. Field work by $\mathrm{R}$ Larson identified an existing cable from MCC-2 in 241-S271 to the pump control cabinet at tank S-108. This cable can be extended from S-108 to S-109 using Yellow Jacket. The existing, direct burial cable is $3 / C$ \#10AWG (NEC rated for $30 A$ ).

3. MCC-2, cubicle E5 has 30 A F.S. with 10 amp fuses and a motor starter for a $1 \frac{1}{2}$ hp jet pump at $S-108$ (now out of service.) To use this, replace the existing $10 \mathrm{~A}$ fuses with $30 \mathrm{~A}$ fuses and bypass the motor starter which is not needed in this installation.

4. $V_{d}$ (Voltage drop) $=($ Resistance $/ K f t)(L$ in $K f t)$ (Current) (square root 3 )

Per NEC table 8: \#10AWG $=1.24 \mathrm{ohms} / \mathrm{K} \mathrm{ft}$ $\# 8 A W G=.778$ ohms $/ \mathrm{K} \mathrm{ft}$ \#6AWG $=.491 \mathrm{ohms} / \mathrm{K} \mathrm{ft}$

$V_{d}$ from MCC to S-108 based upon $\approx 300^{\prime}$ direct burial cable length.

$V_{d}=(1.24)(.3)(30)(1.73)=19.3 \mathrm{~V}$ This is too high (should be within $3 \%$ of $480=14.4 \mathrm{~V})$

EXCEPTION: In order to evaluate the acceptability of the existing \#10AWG cable, re-calculate the voltage drop using the actual load.

$V_{d}=(1.24)(.3)(15)(1.73)=9.7$ Acceptable.

$V_{d}$ from S-108 to S-109 using $\approx 150^{\prime}$ and $3 / C$ \#8AWG Tray cable.

$V_{d}=(.778)(.15)(15)(1.73)=3.0 \mathrm{~V}$

Total voltage drop $\approx 12.7$ volts which is acceptable. 
HNF-SD-WM-DA-236

Rev, 0

\section{1-SX-102 \& 103 SALTWELL EXHAUSTER ELECTRICAL INSTALLATION}

1. Install 500 CFM exhauster to service SX-102 \& 103.

2. Field work by R Larson identified existing cable from MCC-1 in 241-S-271 to the pump control panel at tank $S X-103$. This cable is \#6AWG (NEC rated for 65 amps) from MCC-1 cubicle C1. The cable can be extended from the pump control panel to the exhauster using Yellow Jacket.

3. MCC-1 cubicle $\mathrm{Cl}$ is equipped with a 100A frame circuit breaker rated at 15A. This can be replaced with a unit rated for $60 \mathrm{~A}$ and the cable separated at the pump control panel using fused disconnects to provide both the existing load and the new exhauster load.

4. $V_{d}$ (Voltage drop) $=($ Resistance $/ K f t)(L$ in $K f t)$ (Current) (square root 3 )

Per NEC table 8: \#IOAWG $=1.24 \mathrm{ohms} / \mathrm{K} \mathrm{ft}$ \#8AWG $=.778$ ohms $/ \mathrm{K} \mathrm{ft}$ $\# 6 A W G=.491$ ohms $/ \mathrm{K} \mathrm{ft}$

$V_{d}$ from MCC to S-103 based upon $\approx 400^{\prime}$ direct burial cable length.

$V_{d}=(.491)(.4)(60)(1.73)=20.4 \mathrm{~V}$ This is too high (should be within $3 \%$ of $480=14.4 \mathrm{~V})$

EXCEPTION: In order to evaluate the acceptability of the existing \#6AWG cable, re-calculate the voltage drop using the actual load. (15 for exhauster and 15 existing load.)

$V_{d}=(.491)(.4)(30)(1.73)=10.2$ Acceptable.

$V_{d}$ from S-103 JB to exhuaster using $\approx 50^{\prime}$ and $3 / C$ \#8AWG Tray cable.

$V_{d}=(.778)(.05)(15)(1.73)=1.0 \mathrm{~V}$

Total voltage drop $\approx 11.2$ volts which is acceptable. 
1. 500 CFM exhauster to service BY 105 \& 106.

2. Field work identified existing welding receptacle at the central switching station adjacent to tanks 105 and 106 . This welding receptacle is fed from a fused disconnect switch mounted in the central switching station.

3. Remove the existing 20 Amp fuses from the fused disconnect switch and replace with 30 amp fuses. Disconnect the existing welding receptacle and connect the power cable for the exhauster to the fused disconnect switch.

4. $\quad V_{d}$ (Voltage drop) $=($ Resistance $/ K f t)$ ( $L$ in $\left.K f t\right)$ (Current) (square root 3 )

Per NEC table 8: \#10AWG $=1.24 \mathrm{ohms} / \mathrm{K} \mathrm{ft}$ \#8AWG $=.778 \mathrm{ohms} / \mathrm{K} \mathrm{ft}$ \#6AWG $=.491 \mathrm{ohms} / \mathrm{K} \mathrm{ft}$

$V_{d}$ from fused disconnect to the exhuaster at $B X-105$ at $\approx 100$ feet of $3 \%$ \#8 AWG cable.

$V_{d}=(.778)(.1)(30)(1.73)=4.0 \mathrm{~V}$ Acceptab7e 
241-S-107 and 241-S-101 \& 102 SALTWELL EXHAUSTERS ELECTRICAL INSTALLATION

1. Instal1 1000 CFM exhauster to service tank S-107 and a 1000 CFM exhauster to service tanks S-101 \& 102. (60 amp per unit)

2. There is no power available near either of the subject locations. It was decided to instail new raceway and circuit from the 241-S-171 MCC \#1 into $S$ farm. The transfer Tine berm is to the east of the 271 building. The new raceway will be buried adjacent to the 271 building to accomodate the existing traffic pattern. After the traffic area is crossed, the conduit will be installed on precast footings. It is the intent of the design to install the conduit adjacent to the berm to provide protection for the conduit.

3. MCC \#1 cubicle \#B3 is out of service. Replace the existing 100AF, 15AT circuit breaker with 150AF, 125AT (Cutler Hammer \#FDB3125) to provide up to 120 amp service for the exhausters.

4. $\quad V_{d}($ Voltage drop) $=($ Resistance $/ \mathrm{Kft})(\mathrm{L}$ in $\mathrm{Kft})$ (Current) (square root 3 ) Per NEC table 8: \#6AWG $=.491$ ohms $/ \mathrm{K} \mathrm{ft}$ \#1 AWG $=.154 \mathrm{ohm} / \mathrm{K} \mathrm{ft}$

$V_{d 1}$ from MCC to the pull box adjacent to tank S-107. (Approximately $225^{\prime}$, \#1AWG - rated for 130Amps). Calculated with full load.

$V_{\mathrm{d} 1}=(.154)(.225)(120)(1.73)=7.2 \mathrm{~V}$

$V_{d 2}$ from 5107 pu11 box to pul1 box at S-101. (Approximateluy 225', \#1 $A W G)$ Calculated at 60 amps.

$V_{\mathrm{d} 2}=(.154)(.225)(60)(1.73)=3.6 \mathrm{~V}$

$V_{\mathrm{d} 3}$ from pull box to exhauster unit. (Both units are approximately the same distance $\left[\approx 60^{\prime}\right]$ from the pull box. Both will be wired with \#6 AWG tray cable.)

$V_{\mathrm{d} 3}=(.491)(.06)(60)(1.73)=3.1 \mathrm{~V}$

Total voltage drop from MCC to $S-107 \approx 10.3$ volts (Acceptable)

Total voltage drop from MCC to $S-101 / 102 \approx 13.9$ volts (Acceptable) 